UNIVERSITY OF KWAZULU-NATAL

ENZYMATIC ACTIVATION OF A PEPTIDE FUNCTIONALISED GOLD NANOPARTICLE SYSTEM FOR PRODRUG DELIVERY

by

LAUREN KARA PIETERSEN

2009 


\section{ENZYMATIC ACTIVATION OF A PEPTIDE FUNCTIONALISED GOLD NANOPARTICLE SYSTEM FOR PRODRUG DELIVERY}

by

\section{LAUREN KARA PIETERSEN}

Submitted in fulfilment of the academic requirements for the degree of Masters in Science in the School of Chemistry, University of KwaZulu-Natal, Durban.

November 2009

As the candidates supervisors we have/have not approved this thesis/dissertation for submission.

Supervisor:

Signed:

Name:

Date:

Co-supervisor

Signed:

Name:

Date:

Co-supervisor

Signed:

Name:

Date:

Co-supervisor

Signed:

Name:

Date: 


\begin{abstract}
Traditional chemotherapeutic drugs are often restricted by severe side effects and a lack of specificity. An approach aimed at improving the selectivity of cancer drugs includes the use of prodrugs that can be selectively activated in tumour tissue (tumour-activated prodrugs). Peptide prodrugs cleavable by proteases in the tumour environment have been widely explored to improve the therapeutic index of cytotoxic drugs. In this study we demonstrate that the cell surface proteases over expressed in tumour cells are capable of cleaving the peptide CKAFKRK attached to gold nanoparticles (GNPs). This proteolic cleavage exposes the potential cytotoxic "agent" to the tumour cell. As these proteases are not over expressed in healthy cells, the prodrug will take much longer to be activated. A simple system is developed in this study as a proof of concept by replacing the cytotoxic agent with a fluorophore. An enhanced fluorescence emission is expected on exposure of the peptide functionalised GNP to the tumour cells, whereas a constant level of emission is anticipated on exposure to the healthy cell line.
\end{abstract}




\section{PREFACE}

The experimental work described in this dissertation was carried out in the School of Chemistry and Department of Biochemistry, University of KwaZulu-Natal, Durban, from January 2008 to October 2009, under the supervision of Dr. Thavendran Govender, Prof. Gert Kruger, Dr. Glenn Maguire and Dr. Patrick Govender.

These studies represent original work by the author and have not otherwise been submitted in any form for any degree to any tertiary institution. Where use has been made of work of others it is duly acknowledged in the text. 


\section{DECLARATION 1 - PLAGIARISM}

I, Lauren Kara Pietersen, declare that:

- The research reported in this thesis, except where otherwise indicated, is my original research.

- this thesis has not been submitted for any degree or examination at any other university,

- this thesis does not contain other persons data, pictures, graphs, or other information, unless specifically acknowledged as being sourced from other persons ${ }^{e e}$,

- this thesis does not contain other persons ${ }^{\text {ee }}$ writing, unless specifically acknowledged as being sourced from other researchers. Where other written sources have been quoted, then:

0 their words have been re-written but the general information attributed to them has been referenced,

○ where their exact words have been used, then their writing has been placed in italics, placed inside quotation marks, and referenced,

- this thesis does not contain text, graphics or tables copied and pasted from the Internet, unless specifically acknowledged, and the source being detailed in the thesis and in the references section.

Signed:

Date: 


\section{DECLARATION 2 - PUBLICATIONS}

DETAILS OF CONTRIBUTION TO PUBLICATIONS that form part and/or include research presented in this thesis (include publications submitted and give details of the contributions of each author to the experimental work and writing of each publication).

\section{Publication 1}

Peptide functionalised gold nanoparticles as potential time released prodrug carriers.

Authors: Lauren K. Pietersen, Patrick Govender, Hendrik G. Kruger, Glenn E. M. Maguire, and Thavendran Govender ${ }^{\mathrm{a}}$

\section{Publication 2}

Enzymatic Activation of a Peptide Functionalised Gold Nanoparticle System for Prodrug Delivery.

Authors: Lauren K. Pietersen, Patrick Govender, Hendrik G. Kruger, Glenn E. M. Maguire, James Wesly-Smith and Thavendran Govender ${ }^{\mathrm{a}}$

Signed: Date: 


\section{TABLE OF CONTENTS}

1.

1.1.

1.2.

1.2.1.

1.2.2.

1.2.3.

1.3.

2.

2.1.

2.2 .

2.2.1.

2.2.2.

2.3.

2.3.1.

2.3.2.

2.3.3.

2.4.

2.5 .

2.6.

3.

3.1 .

3.2.

3.2.1.

3.2.1.1.

CHAPTER 1: INTRODUCTION

1

GENERAL BACKGROUND

1

TARGETING .

2

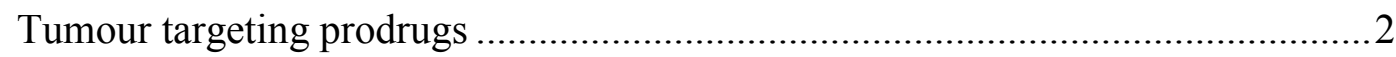

Activation of prodrugs by the serine protease plasmin......................................... 3

Colloidal nanoparticles as delivery vehicles of anticancer drugs ...........................3

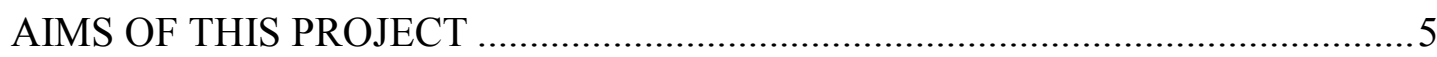

CHAPTER 2: GOLD NANOPARTICLES ..................................................9

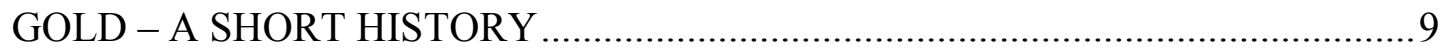

FORMATION AND STABILIZATION OF GNPS …............................................ 11

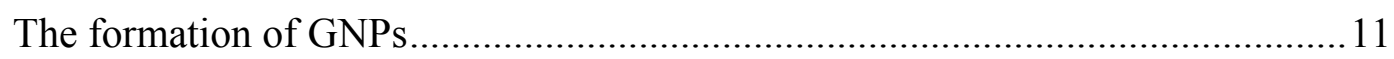

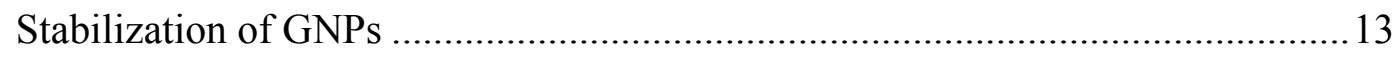

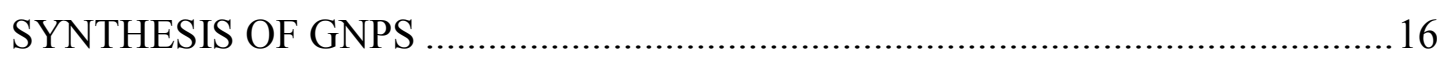

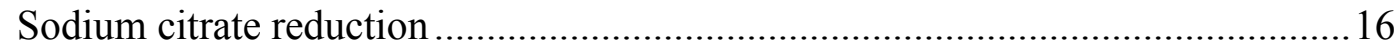

Brust method of synthesis of thiol derivatised GNPs by biphasic reduction......... 17

Sodium borohydride method.

OPTICAL PROPERTIES OF GNPS

19

CHARACTERIZATION OF GNPS

.22

BIOLOGICAL APPLICATIONS OF GNPS

22

CHAPTER 3: PEPTIDE CHEMISTRY

A GENERAL INTRODUCTION .24

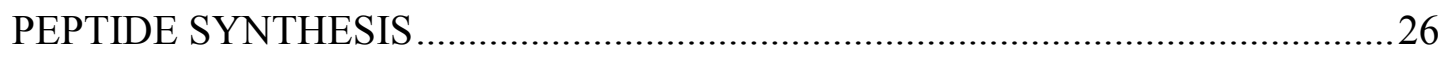

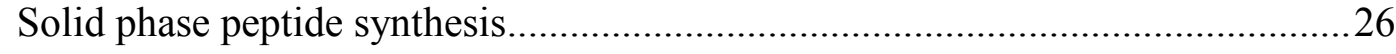

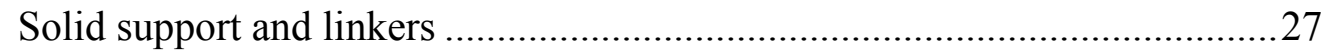


Time taken for complete cellular uptake of peptide capped GNPs and verification of mechanism of internalization 


\subsection{2.}

7.2.2.1.

Attachment of peptides to sodium citrate stabilised GNPs.

Chromatograph 1: LC-MS chromatograph of the peptide GAFK.

Chromatograph 2: LC-MS chromatograph of the peptide CKAFKRK........................................ 140

Spectrum 4: $\quad$ Mass spectrum of CKAFKRK peptide.

Spectrum 5: $\quad$ High resolution mass spectrum of peptide CKAFKRK.

Spectrum 6:

High resolution mass spectrum of peptide CKAFKRKCoum.

Spectrum 8:

High resolution mass spectrum of $\mathrm{C}(\mathrm{KAFKRK})_{3}$ peptide.

Chromatograph 3: LC-MS chromatograph of the peptide CGAFKGGCoum............................... 143

Spectrum 9: $\quad$ Mass spectrum of the peptide CGAFKGGCoum. 
9.

Spectrum 11:

Spectrum 12:

Spectrum 13:

Spectrum 14:

Spectrum 15:

Spectrum 16:

Spectrum 17:

Spectrum 18:

Spectrum 19:

Spectrum 20:

Spectrum 21:

Spectrum 22:

Spectrum 23:

Spectrum 24:

Spectrum 25:

Spectrum 26:

Spectrum 27:
Spectrum 28:

APPENDIX 2: CHARACTERIZATION OF GNPS 145

ATR-FTIR spectrum of CKCoum. 146

ATR-FTIR spectrum of Au-CKCoum. 146

ATR-FTIR spectrum of CGAFKGGCoum. 147 ATR-FTIR spectrum of Au-CGAFKGGCoum. 147

ATR-FTIR spectrum of C(KAFKRK) ${ }_{3}$ Coum................................................. 148

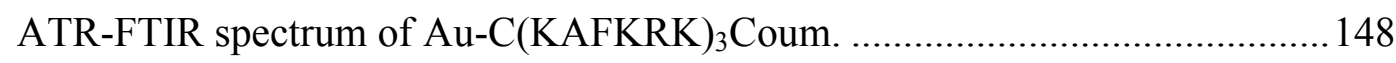

ATR-FTIR spectrum of CKAFKRKCoum.................................................... 149

ATR-FTIR spectrum of Au-CKAFKRKCoum. 149

ATR-FTIR spectrum of Au-10\% CKAFKRKCoum:40\% CKAFKRK:50\% $\mathrm{C}(\mathrm{KAFKRK})_{3}$ 150

${ }^{1} \mathrm{H}$ NMR spectrum of CMECoum in $\mathrm{CD}_{3} \mathrm{OD}$. 151

${ }^{1} \mathrm{H}$ NMR spectrum of Au-CMECoum in $\mathrm{CD}_{3} \mathrm{OD}$. 151

${ }^{1} \mathrm{H}$ NMR spectrum of CKCoum in $\mathrm{D}_{2} \mathrm{O}$. 152

${ }^{1} \mathrm{H}$ NMR spectrum of Au-CKCoum in $\mathrm{D}_{2} \mathrm{O}$. 152

${ }^{1} \mathrm{H}$ NMR spectrum of CGAFKGGCoum in $\mathrm{D}_{2} \mathrm{O}$. 153

${ }^{1} \mathrm{H}$ NMR spectrum of in Au-CGAFKGGCoum in $\mathrm{D}_{2} \mathrm{O}$. 153

${ }^{1} \mathrm{H}$ NMR spectrum of $\mathrm{C}(\mathrm{KAFKRK}){ }_{3}$ Coum in $\mathrm{D}_{2} \mathrm{O}$. 154

${ }^{1} \mathrm{H}$ NMR spectrum of Au-C(KAFKRK $)_{3}$ Coum in $\mathrm{D}_{2} \mathrm{O}$. 154

${ }^{1} \mathrm{H}$ NMR spectrum of $\mathrm{Au}-50 \% \mathrm{C}(\mathrm{KAFKRK})_{3}: 40 \% \mathrm{CKAFKRK}: 10 \%$

CKAFKRKCoum in $\mathrm{D}_{2} \mathrm{O}$. 155

10. APPENDIX 3: ENZYMATIC DEGRADATION 156

Spectrum 29: $\quad$ Mass spectrum of Au-C(KAFKRK $)_{3}$ in PBS prior to enzymatic degradation. ...157 Spectrum 30: $\quad$ Mass spectrum of $\mathrm{Au}-\mathrm{C}(\mathrm{KAFKRK})_{3}$ in PBS after enzymatic degradation. 157 Spectrum 31: $\quad$ Mass spectrum of Au-CKAFKRKCoum:C(KAFKRK) $)_{3}(50: 50)$ in PBS prior to enzymatic degradation. 158

11. SUPPLEMENTARY MATERIAL 159

A CD accompanying this dissertation contains the following: 159 Text of Chapters 1-6 and Appendix 1. 


\section{LIST OF ABBREVIATIONS}

A549

A

AA

AcN

$\mathrm{AcOH}$

Ala

Arg

ATP

ATR

$\mathrm{Au}$

Boc

Cys

CKCoum

CME

CFLSM

DCC

DCM

DIC

DIPEA

DMEM

DMF

DMSO

ESI

FCC

Fmoc

FRET
Human lung epithelial cells

Absorbance

Amino acid

Acetonitrile

Acetic acid

Alanine

Arginine

Adenosine triphosphate

Attenuated total reflectance

Gold

$t$-butyloxylcarbonyl

Cysteine

Cysteine lysine Coumarin

Cysteine methyl ester

Confocal fluorescence laser scanning microscope

Dicyclohexylcarbodiimide

Dichloromethane

Diisopropylcarbodiimide

Diisopropyl ethylamine

Dulbecco s minimal essential medium

Dimethylformamide

Dimethylsulfoxide

Electrospray ionisation

Face-centered cubic

9-fluorenylmethoxycarbonyl

Fluorescence resonance energy transfer 
FTIR

Gly

GNP

$\mathrm{HAuCl}_{4}$

HATU

HBTU

HOBT

$\mathrm{HCl}$

HEPES

$\mathrm{HF}$

HPLC

INT

IR

LC-MS

LDH

Lys

MALDI

MCF-7

MDBK

$\mathrm{MeOH}$

MPC

MMPCs

MPS

MTT

$\mathrm{NaBH}_{4}$

$\mathrm{NaCl}$

$\mathrm{NAD}^{+}$
Fourier transform infrared spectroscopy

Glycine

Gold nanoparticles

Gold tetrachloroaurate

2-(1H-7-Azabenzotriazol-1-yl)--1,1,3,3-tetramethyl uronium hexafluorophosphate methanaminium

O-Benzotriazole- $\mathrm{N}, \mathrm{N}, \mathrm{N}^{e e}, \mathrm{~N}^{e e}$-tetramethyl-uronium-hexafluoro-phosphate

1-hydroxy-benzotriazole

Hydrochloric acid

2-[4-(2-hydroxyethyl)-1-piperazinyl] ethanesulfonic acid

Hydrogen fluoride

High pressure liquid chromatography

2-(4-iodophenyl)-3-(4-nitrophenyl)-5-phenyltetrazolium chloride

Infrared

Liquid chromatography mass spectrometry

Lactate dehydrogenase

Lysine

Matrix-assisted laser desorption ionisation

Human adenocarconoma cell line

Madin Darby bovine kidney epithelium cell line

Methanol

Monolayer protected cluster

Mixed monolayer-protected gold clusters

Macrophages of bone marrow

3-(4,5-diethylthiazol-2-yl)-2,5-diphenyltatrazolium bromide

Sodium borohydride

Sodium chloride

Nicotinamide adenine dinucleotide 


\begin{tabular}{|c|c|}
\hline $\mathrm{NADH} / \mathrm{H}^{+}$ & Dihydronicotinamide adenine dinucleotide \\
\hline NMR & Nuclear magnetic resonance \\
\hline $\mathrm{nm}$ & Nanometres \\
\hline NP & Nanoparticle \\
\hline PBS & Phosphate buffered saline \\
\hline Pen strep & Penicillin/streptomycin \\
\hline Phe & Phenylalanine \\
\hline PolyA & PolyAlanine \\
\hline $\mathrm{pI}$ & Isoelectric point \\
\hline Pro & Proline \\
\hline RP-HPLC & Reverse phase high pressure liquid chromatography \\
\hline RPM & Revolutions per minute \\
\hline SAM & Self-assembled monolayers \\
\hline SD & Standard deviation \\
\hline SEM & Scanning electron microscopy \\
\hline SPE & Solid phase extraction \\
\hline SPPS & Solid phase peptide synthesis \\
\hline TAP & Tumour activated prodrugs \\
\hline Tat & Trans-activating transcriptional activator \\
\hline$t \mathrm{Bu}$ & Tertiary butyl \\
\hline TEM & Transmission electron microscope \\
\hline TFA & Trifluoroactetic acid \\
\hline TOAB & Tetraoctylammonium bromide ions \\
\hline TOF & Time of flight \\
\hline Trt & Trityl \\
\hline UV-vis & Ultraviolet-visible \\
\hline
\end{tabular}




\section{ACKNOWLEDGEMENTS}

First and foremost I wish to convey my deepest appreciation to my supervisors Dr. Thavendran Govender for his support, enthusiasm and being a great source of inspiration, Prof. Gert Kruger for his constant and steady supervision, invaluable input and financial support, Dr. Glenn Maguire for his eagerness to assist and exceptional knowledge of chemistry and Dr. Patrick Govender, for his help in all biological aspects of the project.

I am very grateful to my fellow colleagues at the University of KwaZulu-Natal for being helpful and kind. I am especially grateful to Karen Muthusamy, for all her assistance in biological testing, for dedicating her time to read and comment on the draft copies of my thesis and importantly, and for always being willing to lend a helping hand.

A special thanks to Dr. James Wesley-Smith of the TEM unit for his assistance in the use of the TEM instrument and in the preparation and analysis of my samples.

I am as ever, especially indebted to my parents, Desmond and Colleen Pietersen for their love and support throughout my life, providing me with my every need, for always standing by me in all of my endeavours and being my solace during all hardships. I am very thankful to Donald Lamont, for allowing me to vent, bringing me comfort and making me laugh. I wish to thank my dearest friend, Michelle Bruno, for just listening and being there. 


\section{CHAPTER 1: INTRODUCTION}

This is a multidisciplinary study (Chemistry and Biochemistry). It is not possible to adhere to the usual format of both disciplines in this dissertation. It is also difficult to satisfy the expectation of the expert reader in terms of the depth of discussion of all aspects involved in this study. This dissertation was written from a chemistry perspective. The format of this thesis and the references are based on standard practice in the School of Chemistry. Since the study was performed from a chemistry background, some basic chemical knowledge is assumed. For the convenience of nonbiologists/biochemists, footnotes are used to explain and define various biochemical concepts that may seem standard knowledge to people in the field.

\subsection{GENERAL BACKGROUND}

Cancer is a life threatening illness that affects millions of people worldwide and is one of the most feared diseases to date.

Cancer is able to affect many organs in the human body. ${ }^{\mathbf{1 , 2}}$ These organs are made up of cells that divide and multiply when required by the body and this process of cell division is strictly regulated. ${ }^{3}$ Sometimes, however, the regulatory mechanism is compromised and the affected cells continue to multiply in an uncontrolled fashion, resulting in a mass or growth called a tumour that could either be considered benign (non-cancerous) or malignant (cancerous). ${ }^{1,3}$ Benign tumours are rarely fatal and do not spread to other parts of the body, whereas the cells within malignant tumours spread the disease by invading neighbouring tissues and organs. ${ }^{3,4}$ The cancerous cells within malignant tumours can also break free from the tumour, enter the blood stream, and spread the disease to other organs, a process known as metastasis. ${ }^{3}$

There are many causes of cancer, including environmental factors such as viruses, chemicals, radiation, lifestyle (smoking and excessive alcohol consumption), and internal factors such as inherited conditions and hormonal levels. ${ }^{4}$ Presently, treatments available for the disease include surgery, chemotherapy, radiation therapy, immunotherapy and biological therapy. ${ }^{2}$ The chances of successful treatment depend mainly on the type of cancer and how early the cancer was diagnosed. ${ }^{4}$ Factors such as the type and level of metastasis, age and general health determine the choice of treatment and unfortunately no single method is curative in all cases. Often, several therapeutic modalities may be used in order to provide the most complete treatment for the patient. However, 
even this multi-modality approach has limitations, especially with regard to disseminated cancer, since small clusters of malignant cells can easily evade detection and cannot be effectively treated by surgery or radiation therapy. In these cases, chemotherapy remains the only viable treatment method. ${ }^{4}$

The major drawback of current chemotherapeutic agents is their inability to discriminate between normal and tumour cells and in order to prevent toxicity to the host, only limited doses of the therapeutic drugs can be administered. ${ }^{\mathbf{5 - 8}}$ In most cases, however, the doses administered are seldom sufficient to eradicate cancer cells entirely. ${ }^{5}$

\subsection{TARGETING}

Ultimately, the goal of cancer drug research is the development of agents that will selectively attack cancer cells, sparing the normal tissue of the host. One approach to obtain specificity, is molecular targeting that uses drugs that attack molecular pathways responsible for maintaining and propagating the disease, without disrupting the normal functions of cells and tissue. ${ }^{\mathbf{5 , 6}}$ The design of such tumour targeting drugs begins with understanding how cancer cells metastasize and identifying characteristic as well as targetable differences between normal and tumour cells.

\subsubsection{Tumour targeting prodrugs}

These drugs are referred to as prodrugs and are substances administered in an inactive form that are then metabolized in the body to yield the active compound. ${ }^{5,7}$ Prodrugs can be activated by tumour associated antigens ${ }^{\mathrm{a}}$, enzymes or receptors that are over-expressed on cell surfaces and recognised for therapy by a tumour seeking molecule. ${ }^{9,7}$ Such tumour activated prodrugs (TAP) can be selectively activated by several mechanisms, namely selective enzyme expression, hypoxia ${ }^{\mathrm{b}}$, and low extracellular $\mathrm{pH}$ in the vicinity of the tumour. ${ }^{\mathbf{1 0 , 7}}$ The attachment of peptides onto drugs can facilitate the release of the drug upon selective cleavage of the peptides by enzymes known to be produced and secreted preferentially by tumour cells. ${ }^{\mathbf{5}}$ An example of an enzyme preferentially secreted by tumour cells is the serine protease plasmin.

\footnotetext{
${ }^{a}$ An antigen is a substance that prompts the production of antibodies, thereby generating an immune response.

${ }^{\mathrm{b}}$ Hypoxia refers to a condition in which the entire body (generalized hypoxia) or a region of the body (tissue hypoxia) is deprived of an adequate oxygen supply.
} 


\subsubsection{Activation of prodrugs by the serine protease plasmin}

A targetable characteristic of cancer cells is the over proliferation of the serine protease plasminogen activator ${ }^{\mathrm{c}}$. The cleavage of the zymogen, plasminogen, by serine protease plasminogen activator generates the serine protease, plasmin. Small amounts of activator are able to produce significant amounts of plasmin, the activity of which is tightly regulated by normal controls on plasminogen activator. Protease inhibitors are capable of inactivating free plasmin ensuring that the tumour associated plasmin activator is highly localised around the area of the tumour cell.

The generation of plasmin by cancer cells provides an attractive option for the activation of anticancer prodrugs. ${ }^{8}$ An example of such a prodrug system is the incorporation of a tumour specific recognition site within the prodrug, such as the tumour-specific sequence, D-Ala-Phe-Lys, that is selectively recognised by the tumour-associated protease, plasmin. ${ }^{\mathbf{5}}$ In such a system the tripeptide would be cleaved by the enzyme, exposing the cytotoxic agent to the tumour. This carrier or drug delivery vehicle for cytotoxic agents may possess immeasurable value as potential anticancer treatments due to its ability to deliver cytotoxic agents to tumour tissue and cells while protecting the drug from premature activation during transport. ${ }^{\mathbf{6}}$

\subsubsection{Colloidal nanoparticles as delivery vehicles of anticancer drugs}

Nanoparticles (NPs) are sub-micronic $(<1 \mu \mathrm{m})$ colloidal systems. They are envisaged as promising drug carrier systems due to the following attributes:

- Small size,

- ease of synthesis,

- ease of functionalisation,

- high binding capacity,

- non-toxicity,

- stability,

- inertness in the human body,

\footnotetext{
${ }^{c}$ Plasminogen activator is an enzyme that catalyzes the conversion of plasminogen to plasmin, the major enzyme responsible for the breakdown of blood clots.
} 
- NPs less than $50 \mathrm{~nm}$ in diameter, are suitably sized for passive uptake by the leaky vasculature $^{\mathrm{d}}$ of tumour tissue. ${ }^{6}$

The incorporation of anti-tumour drugs onto the surface of NPs has been reported to improve the effectiveness of the drug in the following ways: ${ }^{6}$

- Overcome non-cell and cell based mechanisms of resistance,

- increase the selectivity of the drug to cancer cells,

- reduce the toxicity of the drugs to normal cells,

- increase drug concentrations in cancer tissue,

- enhance antitumor efficacy. ${ }^{6}$

NPs do, however, have the considerable drawback of an extremely short circulation time of only 35 minutes after intravenous administration. This is caused by the massive capture of the NPs by the macrophages of the bone marrow (MPS) organ. ${ }^{\mathbf{5 , 6}}$ As a result, alternate NP systems have been investigated in order to target tumour organs in preference to the MPS area.

Extensive interest has been focussed on so-called stealth particles that are promising candidates as anticancer drugs due to their:

- Prolonged half-life in the blood compartment,

- invisibility to macrophages, ${ }^{6}$

- ability to target many tumours outside the MPS region. ${ }^{6}$

Stealth particles are characterised by a high curvature, extremely small size $(<100 \mathrm{~nm})$ and are differentiated from conventional hydrophobic NPs by their hydrophilic surface. ${ }^{6}$ The hydrophilic coatings such as polyethylene glycol, poloxamines, poloxamers, polysaccharides and peptides, create a layer of hydrophilic and neutral chains at the particle surface which act to repel plasma proteins. These coatings are easily attached to the surface by adsorption of surfactants or by the use of block or branched co-polymers. The latter method of attachment/functionalisation is preferred as it avoids the possibility of rapid coating desorption upon dilution or after contact with blood components. ${ }^{6}$ The functionalisation of gold nanoparticles (GNPs) allows the properties of GNPs to be adjusted to impart the required stability, solubility and selective targeting of the macromolecule.

\footnotetext{
${ }^{\mathrm{d}}$ The network of blood vessels in an organ is referred to as the vasculature of the organ in question. Most cancers have leaky vasculature of the blood capillaries in the tumour that result in defective vascular architecture (broken or incomplete blood capillaries).
} 


\subsection{AIMS OF THIS PROJECT}

The design of a drug delivery system exploiting the differential rates of biochemical reactions in tumour versus healthy cells has not, to the best of our knowledge, been reported. Due to the lack of drug specificity, this project aims at investigating the relationship between the structures of peptides attached to GNPs and their susceptibility toward enzyme degradation, as a novel mechanism for selective cancer drug delivery. This delivery system will be based on the principle that cancer cells are more active than healthy cells in terms of the over proliferation of proteases.

The proposed drug is envisaged to have the following features:

- A timing device that "turns on" the destructive mode of the drug in cancerous cells,

- a "forest" of long peptides protecting the healthy cells from the drug action until the timing device has switched off the drug action and,

- a timing device that "switches" the cancer destructive mode of the drug off at a certain time.

If that can be achieved, a tumour specific drug could be within reach.

The long term (post MSc) goal of this project is to destroy cancer cells by means of a short distance radioactive atom attached to a DNA intercalator. The radioactive DNA intercalator will be attached to a GNP by means of a short peptide sequence. Also attached to the GNP will be a forest of longer peptide chains specifically designed to be consumed by the over expressed proteases in cancer cells. When the GNP prodrug is delivered to a cancerous cell the over expressed proteases will ensure a fast rate of consumption of the protective peptide shield. Once the radioactive intercalator is exposed to the DNA of the cancer cell it would cause irreversible damage to the DNA of the cell leading to a systematic destruction of the cancerous cells.

The half-life of the radionucleotide will be chosen such that it will have decayed to negligent levels by the time the much slower proteolysis of the healthy cells will have exposed the radioactive DNA intercalator. Since a short distance radioactive element will be used, damage to healthy cells will be limited due to the effective shielding of the radiation by the forest of long peptide chains. The theory is illustrated as shown in Figure 1. 


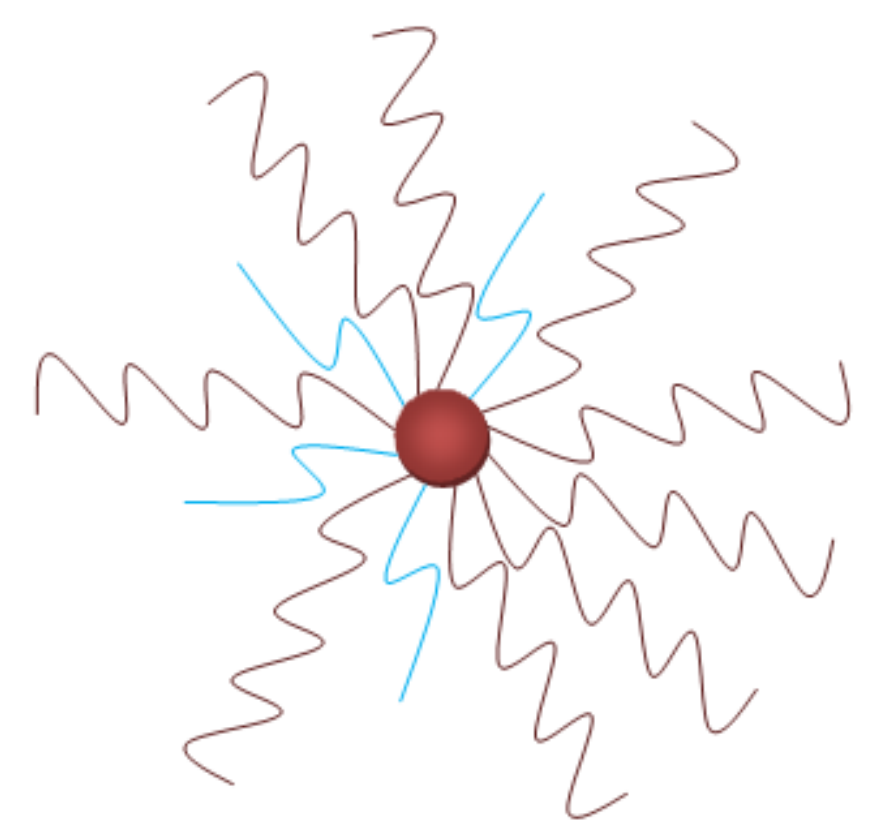

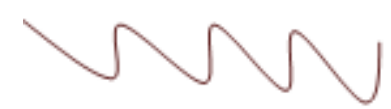
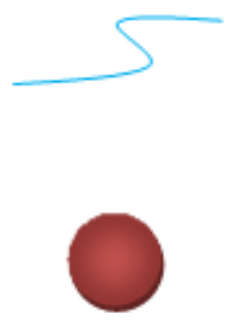

Longest peptide chain of sequence $C A_{n}(K A F K R K)_{n}$.

\section{Shortest peptide chain of sequence $\mathrm{CA}_{\mathrm{n}}(\mathrm{KAFKRK}) \mathrm{Coum}$ with radioactive atom attached.}

\section{Gold nanoparticle.}

Figure 1: Proposed schematic of peptide strands attached to gold NPs.

It was decided to establish proof of concept using a simplified system for this project. Replacing the radioactive part of the prodrug with a fluorescent tag would eliminate the risk factor of working with radioactive nucleotides. It is envisaged that the uptake of the GNP into living cells could be monitored using fluorescent tags. The intensity and wavelength of fluorescence should also change when the forest of longer peptides is removed by the protease reaction. The fluorescent tags should, therefore, also enable determination of the different rates at which cancer cells versus normal cells consume the longer peptides. In turn this will direct the choice of radioactive element to be used in future studies. 
A peptide containing the sequence Cys-Ala $-(\text { Lys-Ala-Phe-Lys-Arg-Lys })_{n}\left[C_{n}(K A F K R K)_{n}\right]$, will be synthesised. The particular amino acid residues were selected on the basis of the following considerations:

- The cysteine residue facilitates the covalent attachment of the peptide sequence to the gold surface,

- the incorporation of the tumour specific sequence D-AFK is necessary for selective cleavage by cancer cells,

- the incorporation of $\mathrm{D}$-Alanine prevents the formation of $\beta$-sheet structures,

- hydrophilic residues, such as lysine and arginine are required to,

○ ensure water solubility,

- facilitate membrane permeability of the peptide capped GNPs, ${ }^{11}$

- hydrophobic D-Alanine and phenylalanine are incorporated to impart the necessary stability to the GNP macromolecule, thereby preventing aggregation of the particles.

Coumarin-3-carboxylic acid, a fluorescent dye, will be attached to the terminal lysine in the shortest peptide strand. The GNPs will be modified with $\mathrm{CA}_{\mathrm{n}}(\mathrm{KAFKRK})$ Coum, and $\mathrm{CA}_{\mathrm{n}}(\mathrm{KAFKRK})_{\mathrm{n}}$. Since the length of the latter is significantly longer than that of the former, the Coumarin will be surrounded by the long peptide strands, providing an outer layer that is required to protect the healthy cells against radiation in a later part of the project (Figure 1). Fluorescence testing will establish the ratio of $\mathrm{CA}_{n}(\mathrm{KAFKRK})$ Coum to $\mathrm{CA}_{\mathrm{n}}(\mathrm{KAFKRK})_{\mathrm{n}}$, as well as the required length of the segment KAFKRK to prevent significant quenching of the fluorophore by the GNPs. Trypsin/versene digestion studies (section 5.6.3., page 77) are expected to reveal the necessary spacing required for complete digestion of the peptide prior to cell testing.

The correct length of the peptide segment $A_{n}$ KAFKRK, necessary to prevent the occurrence of fluorescence resonance energy transfer between the fluorophore and the gold surface, must be elucidated. The ratio of $\mathrm{CA}_{n}(\mathrm{KAFKRK})_{\mathrm{n}}$ and $\mathrm{CA}_{\mathrm{n}}(\mathrm{KAFKRK})$ Coum must be determined such that the fluorescence emission intensity is clearly visible. These constraints will ensure a distinct change in fluorescence intensity when the longer peptide chains are digested to the extent where the fluorescent tag is exposed. This theory will be tested using trypsin/versene digestion studies: It is proposed that after cleavage of the „forest of peptides ${ }^{\text {ee }}$ (long peptide), the shorter peptide will be exposed thereby revealing enhanced fluorescence intensity (section 5.2., page 68). Enzymatic degradation studies should reveal an increase in fluorescence on incubation of malignant cells with protected GNPs as opposed to healthy cells. This would indicate an increased rate of consumption 
of the protective peptides $\mathrm{CA}_{n}(\mathrm{KAFKRK})_{\mathrm{n}}$ by unhealthy cells due to the over proliferation of proteases.

As GNPs play a central role in this project, they will be discussed further in Chapter 2. 


\section{CHAPTER 2: GOLD NANOPARTICLES}

NPs are materials that have a characteristic size of $<100 \mathrm{~nm}$. Interest in NPs has grown tremendously in many fields including chemistry, physics, biology, materials science, catalysis, engineering and computer science due to the unique chemical, electrical, magnetic and optical properties. As a result GNPs are the most widely investigated element in nanoscale science. ${ }^{\mathbf{1 2}}$

\subsection{GOLD - A SHORT HISTORY}

Gold has a legendary status of being the most noble of all metals as it never tarnishes on exposure to the atmosphere and is unaffected by most chemicals. ${ }^{13}$ It has been used as a monetary standard throughout the history of mankind, and has been the source of many beautiful historical artefacts, works of art and jewellery. ${ }^{14}$

People have unknowingly used gold in its sub-micronic form for centuries with the oldest documented example being its use for decorative purposes in the Lycurgus cup from the fourth century AD. ${ }^{14}$ In direct light the cup resembles jade with an opaque greenish-yellow tone, but when transmitted light shines through the glass it turns into a translucent ruby colour (Figure 2 AB). The unique optical properties of the cup are a result of the presence of colloidal gold (Figure 2 C) and this antiquity represents one of the outstanding achievements of the ancient Roman glass industry. ${ }^{14}$ 

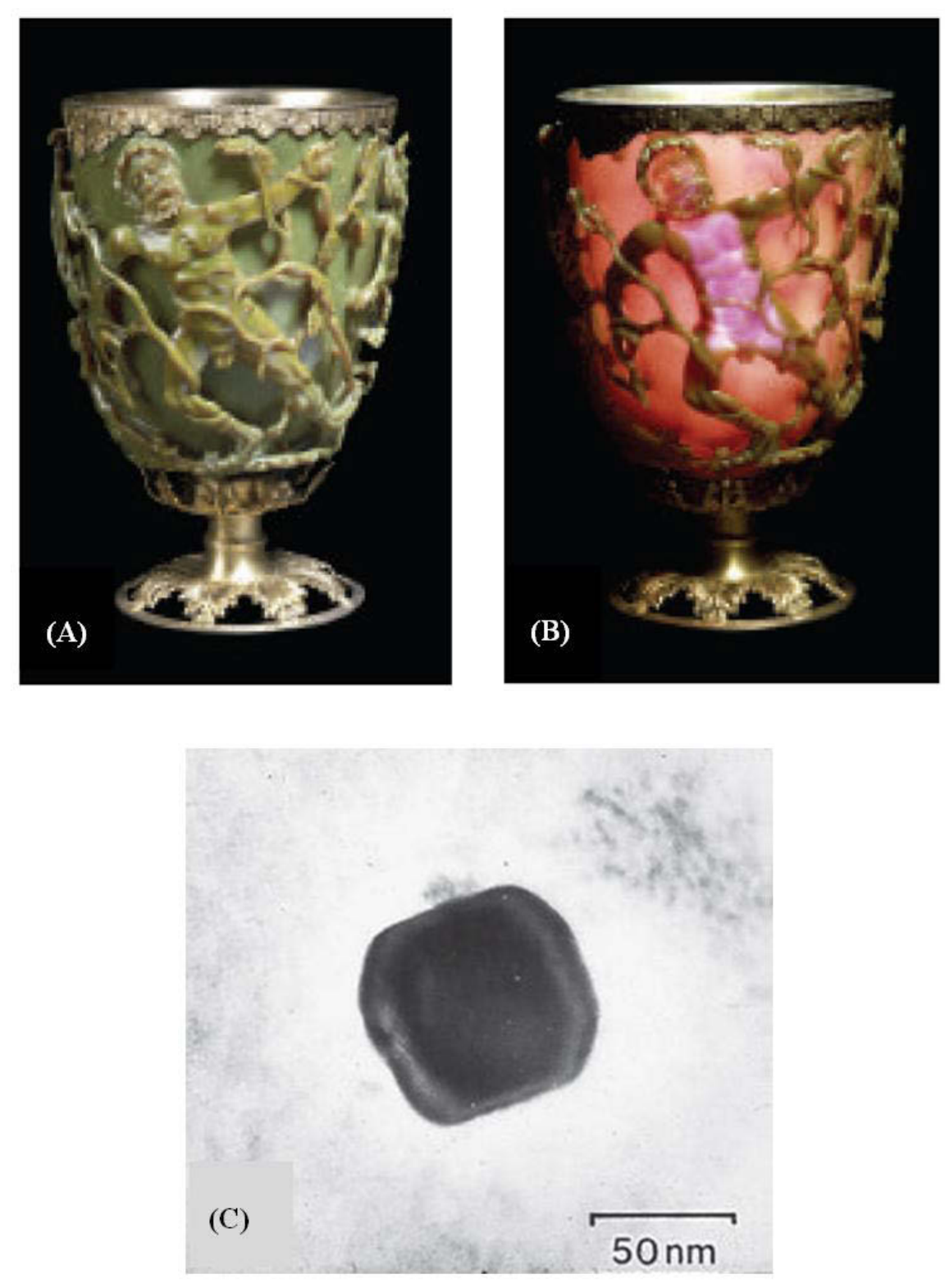

Figure 2: Lycurgus cup, Roman Era ( $4^{\text {th }}$ century A.D). It appears green in reflected light (A) and red in transmitted light (B). It contains gold and silver particles approximately $70 \mathrm{~nm}$ in a ratio of 1:14 (C). The spectral colour effects are due to these nanocrystals. Figure from the British Museum, http://www.thebritishmuseum.ac.uk (Accessed 16 August 2009).

It was not until 1857 that Michael Faraday reported a systematic study of the synthesis and colour of colloidal gold. Faradayes work was at the frontier of colloidal science and led to the broadening interest in colloidal gold during the last quarter of the $19^{\text {th }}$ and beginning of the $20^{\text {th }}$ century. ${ }^{\mathbf{1 3}}$ Presently, GNPs are investigated in the field of material science, catalysis, surface science and synthesis due to their many attributes, such as:

- Ease of synthesis,

- ease of modification in terms of size and shape, ${ }^{\mathbf{1 5}}$ 
- ease of functionalisation,

- small size,

- stability,

- interesting optical and electrical properties: GNPs have enormous visible light molar absorbtivity $\left(\varepsilon>10^{5} \mathrm{~mol}^{-1} \mathrm{~cm}^{-1}\right)$, which is several magnitudes higher than even the best organic dye molecule (see section 2.4., page 19, for details),

- ability to carry high payloads of drug, ${ }^{17}$

- inherent lack of toxicity to human cells: Shukla (2005) ${ }^{\mathbf{1 8}}$ and Connor (2005) ${ }^{\mathbf{1 9}}$ have found that in spite of efficient uptake into human cells by endocytosis, GNPs capped with hydrophilic coatings show little cytotoxicity.

\subsection{FORMATION AND STABILIZATION OF GNPS}

Since the pioneering work of Faraday, extensive research has been conducted on GNPs with the intention of developing simplified methods for the formation and stabilization of gold NPs.

\subsubsection{The formation of GNPs}

Over the last 20 years, a variety of methods have been introduced for synthesising GNPs with a unified goal of producing NPs that:

- Are of a monodisperse ${ }^{\mathrm{e}}$ nature,

- $\quad$ are controlled and uniform in diameter,

- remain colloidally stable .

Most reports follow a similar regime to produce atomic gold, and make use of a reducing agent to donate electrons to the positively charged gold ions in solution. ${ }^{\mathbf{1 1}}$ This process is illustrated in the following equation.

$$
\mathrm{Au}^{3+}+3 \mathrm{e}^{-} \longrightarrow \mathrm{Au}^{0}
$$

\footnotetext{
${ }^{\mathrm{e}}$ A collection of particles are referred to as monodisperse if they all have the same size and shape.

${ }^{\mathrm{f}}$ Remain in suspension and resists settling.
} 
Reducing agents used in this process include sodium citrate (section 2.3.1., page 16), sodium borohydride (section 2.3.3., page 19) and sodium thiocyanate. ${ }^{\mathbf{2 0}}$ The process of reduction of gold tetrachloroaurate $\left(\mathrm{HAuCl}_{4}\right)$ to atomic gold is illustrated in Figure 3.

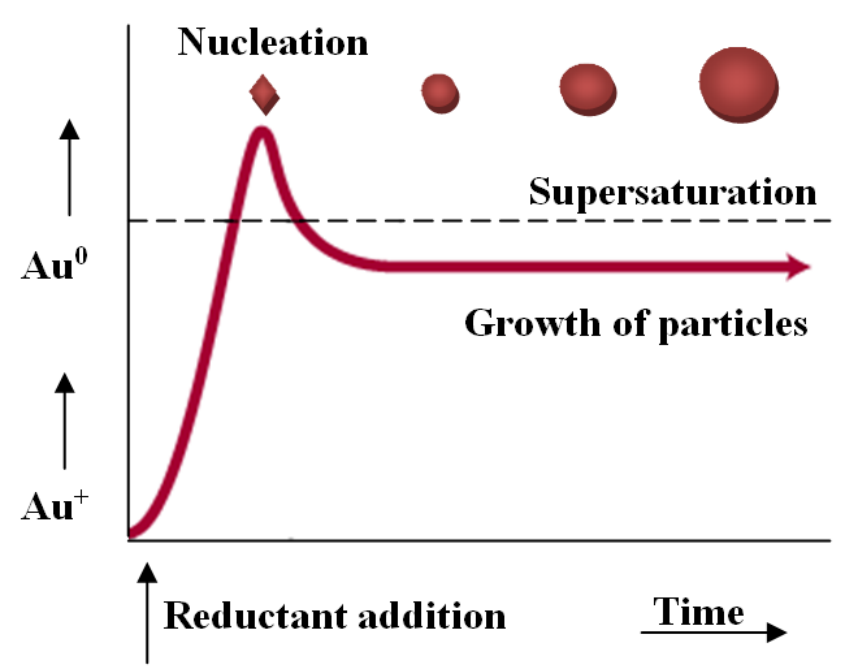

Figure 3: Nucleation of GNPs. Redrawn from Chandler et al. (2009). ${ }^{\mathbf{2 1}}$

Before the addition of any reducing agent, the solution consists solely of gold ions and as the reducing agent is added, the gold ions are transformed to gold atoms (y axis). After the addition, there is a sharp increase in the gold atom content until super-saturation is reached. Aggregation follows super-saturation in a process called nucleation in order to decrease the super-saturation of the gold atoms in solution. The remaining gold atoms continue to bind to the nucleation sites under an energy-lowering gradient to efficiently remove all the atoms from the solution. The number of particles that grow in solution is defined by the number of nuclei that initially form. As the concentration of reducing agent is increased, the number of nuclei that form increases, resulting in the formation of smaller GNP sizes. ${ }^{21}$ It is thus imperative to establish the ideal manufacturing conditions such that all nucleation sites are formed instantaneously and simultaneously. This will result in GNPs of the same size (monodispersity).

The synthesis of GNPs under conditions that are not ideal often results in irreproducible GNP sizes that yield unstable gold conjugates. ${ }^{21}$ These poorly dispersed GNP solutions are easily identified by their bluish or purple colour as opposed to the cherry red colour that indicates a well prepared solution as shown in Figure $4 .^{\mathbf{2 2}}$ Under conditions of extreme aggregation, the solution becomes colourless and a black precipitate can be observed. ${ }^{\mathbf{2 2}}$ 
(A)
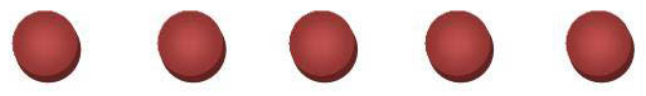

(B)

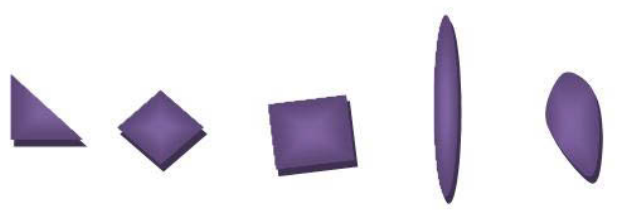

Figure 4: Illustration of different qualities/geometries of GNPs. A) Well manufactured gold: Cherry red, excellent monodispersity, all spherical. B) Poorly manufactured gold: Purple, poor monodispersity, odd shapes. Redrawn from Chandler et al. (2009). ${ }^{\mathbf{2 1}}$

The optical properties of GNPs depend strongly on their size and shape. These effects are the result of a phenomenon known as surface plasmon resonance, described further in section 2.4. (page 19).

\subsubsection{Stabilization of GNPs}

The stabilization of GNPs can be achieved by two methods:

- Electrostatic stabilization,

- steric hindrance. $^{23,24}$

Electrostatic stabilization involves the creation of an electrical double layer arising from the ions adsorbed on the particle surface. The GNPs are suspended and prevented from agglomeration by this negative charge layer surrounding each particle. This charge layer arises from the residual negative ions in solution as shown in Figure 5.

\section{Electrostatic repulsion}

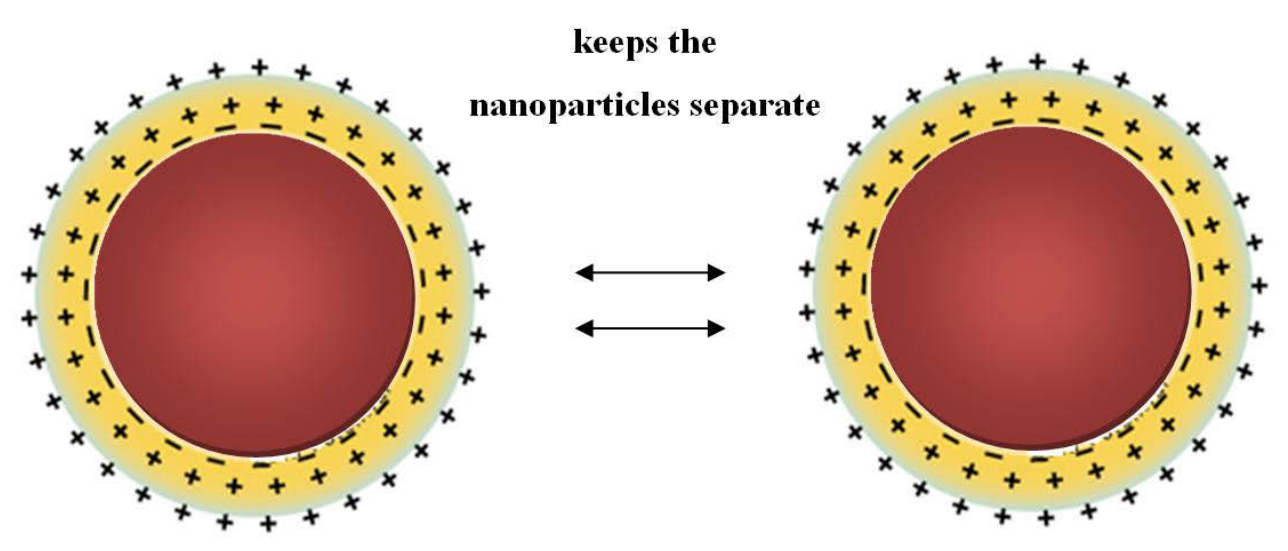

Figure 5: Electrostatic hindrance due to the coulombic repulsion between the adsorbed ions on the particle surface. Redrawn from Chandler et al. (2009). ${ }^{\mathbf{2 1}}$ 
This form of stabilization should prevent aggregation of the particles as long as the electric potential associated with the double layer is sufficiently high. ${ }^{23}$ However, over extended periods of time in solution these electrostatically stabilised particles are increasingly more susceptible to oxidation or the attractive interparticle Van der Waales forces. As a result the particles may aggregate and precipitate from solution. ${ }^{\mathbf{2 5}}$

To prevent this, the electrostatic stabilization can be further enhanced by the incorporation of stabilizing agents such as phosphine, thiol, carboxylate, or amine containing molecules onto the GNP monolayer. ${ }^{25}$ This form of stabilization is referred to as stabilization via steric hindrance and can be achieved by the adsorption of large molecules on the particle surface (Figure 6). ${ }^{\mathbf{2 0}}$

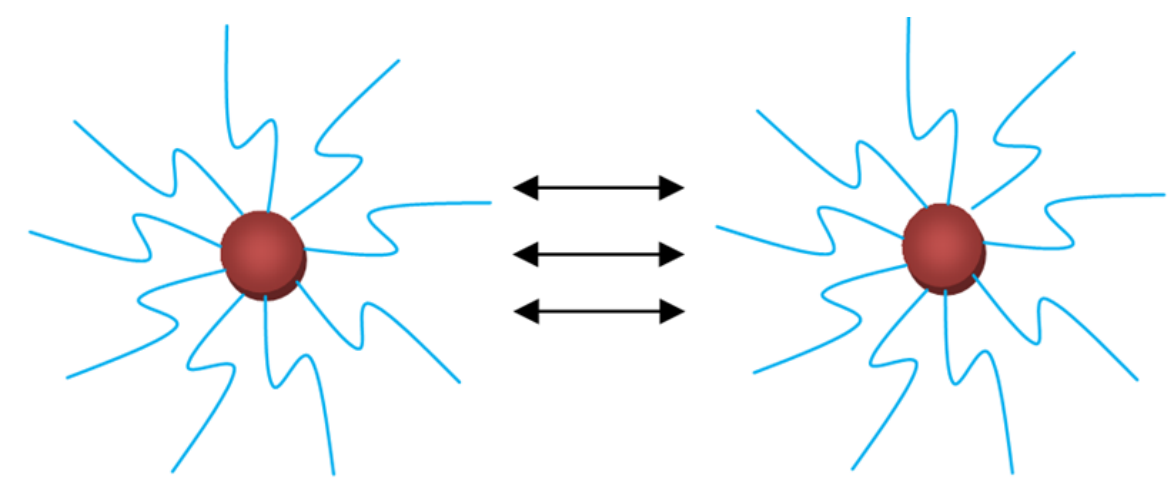

Figure 6: GNPs separated via steric hindrance. Redrawn from Chandler el al. (2009). ${ }^{\mathbf{2 1}}$

Particles stabilised in this manner are known as monolayer protected clusters (MPCs) or self assembled monolayers (SAMs). ${ }^{\mathbf{2 6}}$ In the case of electrostatically stabilised GNPs, ligands can be incorporated onto the GNP surface by means of an exchange reaction with the electrostatically stabilised NP (refer to section 2.3.1., page 16, for details on synthesis). Ligands can also be incorporated onto the GNPs monolayers by reductively generating the NPs in the presence of the ligand (refer to section 2.3.3., page 19, for details on synthesis). The ligands are adsorbed onto the surface of the gold via the following mechanisms as shown in Figure 7.

1. Charge attraction of the positively charged ligand to the negatively charged gold particle,

2. hydrophobic adsorption between certain ligand residues to the particle surface,

3. dative/covalent bonding between sulfur residues of the ligand and the gold particle. ${ }^{21}$ 


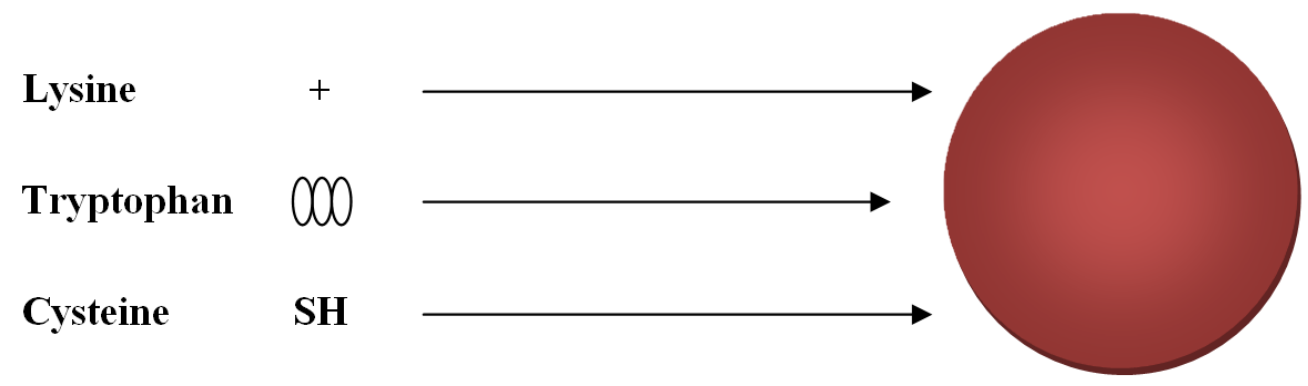

Figure 7: Binding forces between amino acids and a gold particle. Lysine is attached to the surface of the gold particles through mechanism 1, cysteine is attached via mechanism 2 and tryptophan is attached by means of mechanism 3. Figure redrawn from Chandler et al. (2009). ${ }^{\mathbf{2 1}}$

Both methods of GNP stabilization allow the ability to select the ligand attached to the GNP such as proteins and nucleic acids. This introduces a means by which the NPs may be directed to a target site in a body or in a cell for detection, diagnosis, or therapeutic purposes. The general structure of the ligands and their attachment to the gold can be seen in Figure 8. The hydrophilicity of the spacer and tailgroup greatly affects the overall water solubility of the capped GNPs, a prerequisite for most biological studies (refer to section 2.6., page 22).

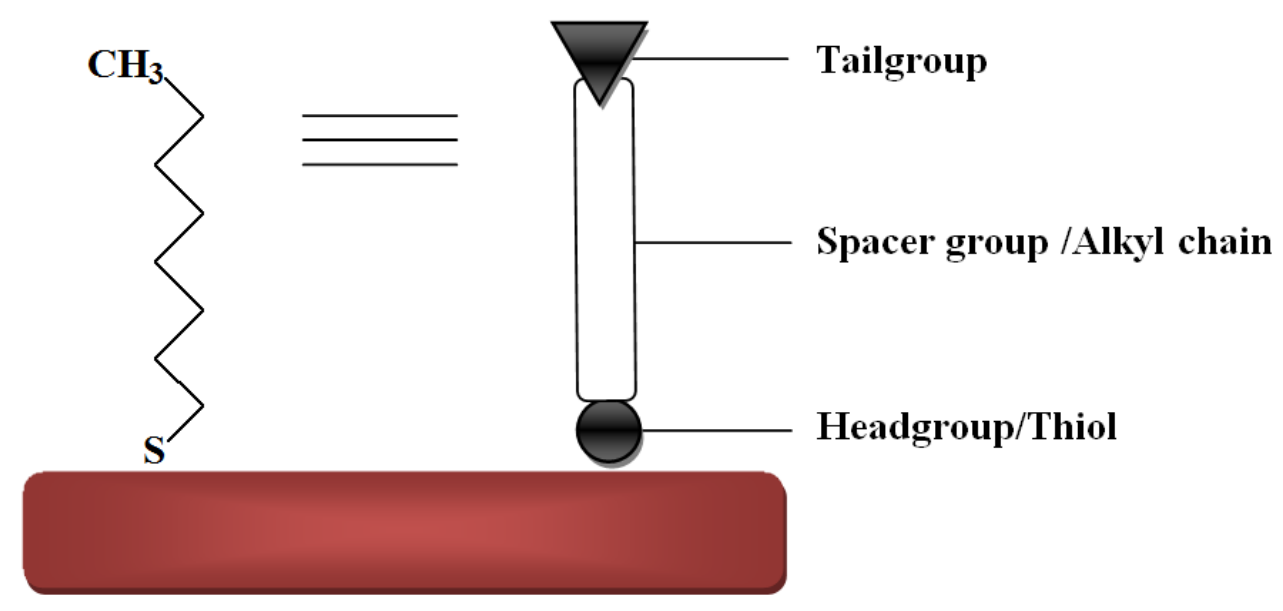

Figure 8: Diagram of an alkanethiol adsorbate on the surface of gold, depicting the tailgroup, alkyl chain and headgroup. Redrawn from Colorado et al. (2001). ${ }^{\mathbf{2 7}}$ 


\subsection{SYNTHESIS OF GNPS}

Many techniques are currently available for the synthesis of GNPs. Three common methods that are used are sodium citrate reduction, the brust and the sodium borohydride method.

\subsubsection{Sodium citrate reduction}

The sodium citrate method, also known as the "Turkevitch" 1850, when Michael Faraday reported a study of the synthesis and properties of GNPs based on the citrate reduction of $\mathrm{HAuCl}_{4}$ to $\mathrm{Au}$ colloids in water. ${ }^{29}$ The procedure entails the following: An aqueous solution of $\mathrm{HAuCl}_{4}$ was brought to boiling point. A reducing agent, trisodium citrate, was rapidly added with stirring under reflux. This agent reduced $\mathrm{Au}(\mathrm{III})^{3+}$ to $\mathrm{Au}(0)$. The initial pale yellow solution immediately turned to bright red indicating the formation of spherical GNPs.

$$
6 \mathrm{AuCl}_{4}+\mathrm{C}_{6} \mathrm{H}_{8} \mathrm{O}_{7}+5 \mathrm{H}_{2} \mathrm{O} \stackrel{\substack{\text { Reflux } \\ 30 \text { mins }}}{\longrightarrow} 6 \mathrm{CO}_{2}+24 \mathrm{Cl}^{-}+6 \mathrm{Au}^{0}+18 \mathrm{H}^{+}
$$

In this procedure, citrate acts as both a reducing and capping agent. Citrate is an effective capping agent as it forms a layer of citrate ions over the GNP surface that induces sufficient electrostatic repulsion between individual particles to ensure that they remain well dispersed in the reaction medium. The Turkevitch method yields $\sim 15 \mathrm{~nm}$ nano-spheres with a narrow size distribution that can be varied by adjusting the concentration of reducing agent to gold salt (the larger the ratio, the smaller the particle size). ${ }^{\mathbf{3 0 , 3 1}}$ Once the colloid is formed, the addition of excess sodium citrate or prolonged heating has no effect on the particle size.

The major drawback of this procedure is due to the electrostatic nature of stabilization. The electrostatic-stabilised NPs can irreversibly aggregate, complicating further handling and manipulation of the NPs. ${ }^{29}$ This can however be prevented by the attachment of thiolated linked ligands to the GNPs (Figure 9). 


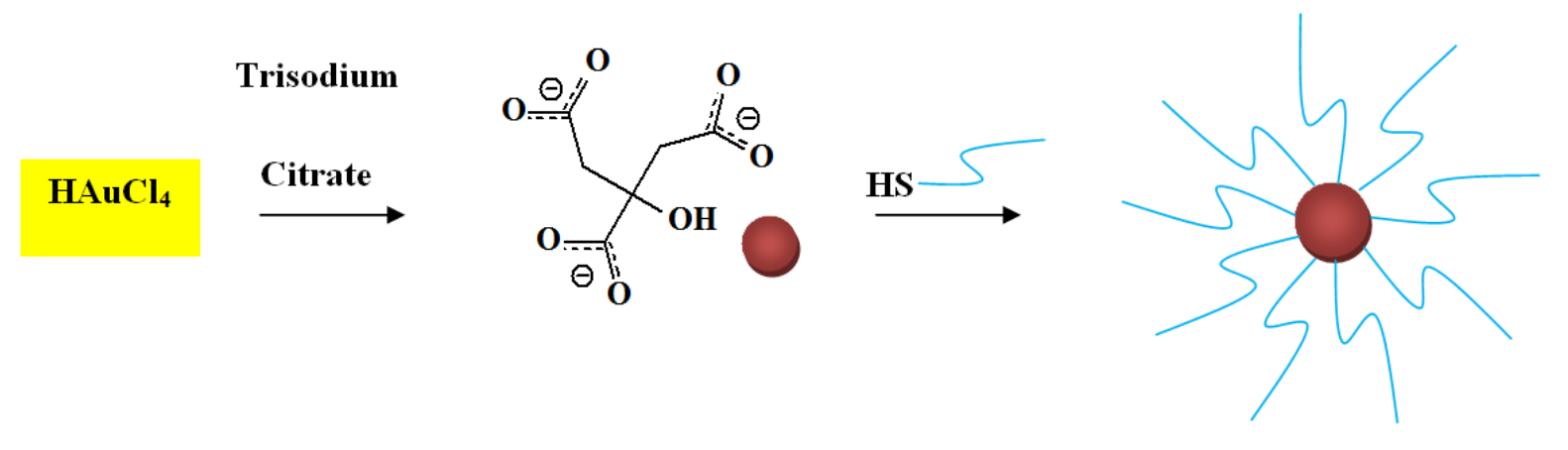

Figure 9: Construction of thiol stabilised MPCs through the sodium citrate reduction method. Redrawn from Hu et al. (2006). ${ }^{\mathbf{2 9}}$

The lack of stability of sodium citrate stabilised GNPs over extended periods has resulted in the discovery of more robust and versatile versions of the synthesis that affords the use of better stabilising agents. In 1994, Brust and Schiffirin reported the synthesis of the first MPCs or SAMs. $^{32}$ The introduction of thiolated molecules improves the stability of the NPs allowing them to be dispersed in various solvents without aggregation. NPs synthesised in this way are particularly appealing as the addition of covalently bonded thiolated molecules endow the NPs with useful catalytic, transport and recognition properties. ${ }^{29}$

\subsubsection{Brust method of synthesis of thiol derivatised GNPs by biphasic reduction}

In 1988, Mathias Brust discovered a novel synthesis of GNPs that affords excellent control and uniformity in size of GNPs. The Brust synthesis is carried out in organic solvents and involves the transfer of tetrachloroaurate anions from an aqueous phase to an organic (toluene) phase by tetraoctylammonium bromide ions (TOAB). The toluene solution is then stirred with sodium borohydride $\left(\mathrm{NaBH}_{4}\right)$ in the presence of alkanethiols. The $\mathrm{NaBH}_{4}$, reduces the $\mathrm{HAuCl}_{4}$, while the alkanethiol molecule capps the $\mathrm{AuCl}_{4}{ }^{-}$to yield stable GNPs (Figure 10). ${ }^{33}$ On addition of the reducing agent, the organic phase rapidly changes colour from orange to deep brown.

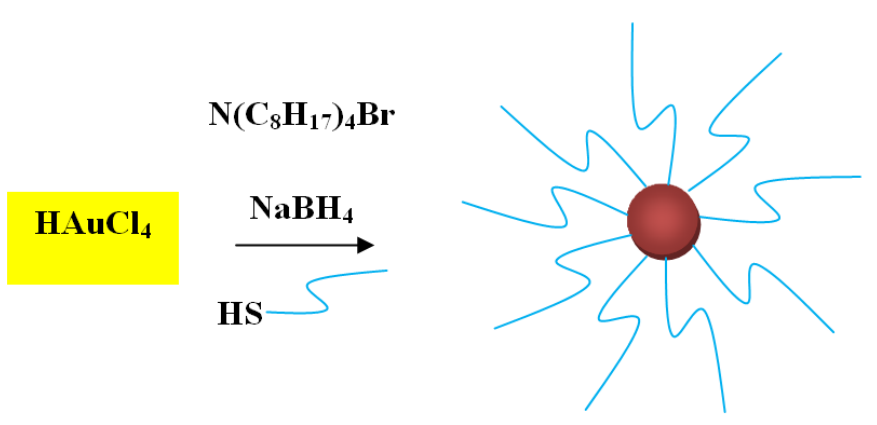

Figure 10: Preparation of thiol stabilised GNPs by the Brust method. 
The two step reaction is shown in the following reactions:

$$
\begin{aligned}
\mathrm{AuCl}_{4}^{-}(\mathrm{aq})+\mathrm{N}^{-}\left(\mathrm{C}_{8} \mathrm{H}_{17}\right)_{4}{ }^{+}\left(\mathrm{C}_{6} \mathrm{H}_{5} \mathrm{Me}\right) & \longrightarrow \mathrm{n}\left(\mathrm{C}_{8} \mathrm{H}_{17}\right)_{4}{ }^{+} \mathrm{AuCl}_{4}^{-}\left(\mathrm{C}_{6} \mathrm{H}_{5} \mathrm{Me}\right) \\
\mathrm{mAuCl}_{4}-\left(\mathrm{C}_{6} \mathrm{H}_{5} \mathrm{Me}\right)+\mathrm{nC}_{12} \mathrm{H}_{25} \mathrm{SH}\left(\mathrm{C}_{6} \mathrm{H}_{6} \mathrm{Me}\right)+3 \mathrm{me}^{-} & \longrightarrow 4 \mathrm{mCl}^{-}(\mathrm{aq})+\left(\mathrm{Au}_{\mathrm{m}}\right)\left(\mathrm{C}_{12} \mathrm{H}_{25} \mathrm{SH}_{\mathrm{n}}\left(\mathrm{C}_{6} \mathrm{H}_{5} \mathrm{Me}\right)\right.
\end{aligned}
$$

The particle sizes can be adjusted to between 1-5 nm depending on the ratio of thiol to gold atoms, with the larger ratios yielding smaller NP diameters. The synthesis of NPs in organic solvents offers several advantages such as:

- Greater control of particle size that in turn results in monodispersed particles,

- can be easily and quickly dried,

- can be easily re-dispersed in a variety of organic solvents.

These MPCees can be further elaborated by the Murray place exchange reactions, whereby ligands can be replaced by other thiol-ended groups to yield mixed monolayer-protected gold clusters (MMPCs) (Figure 11). ${ }^{31}$

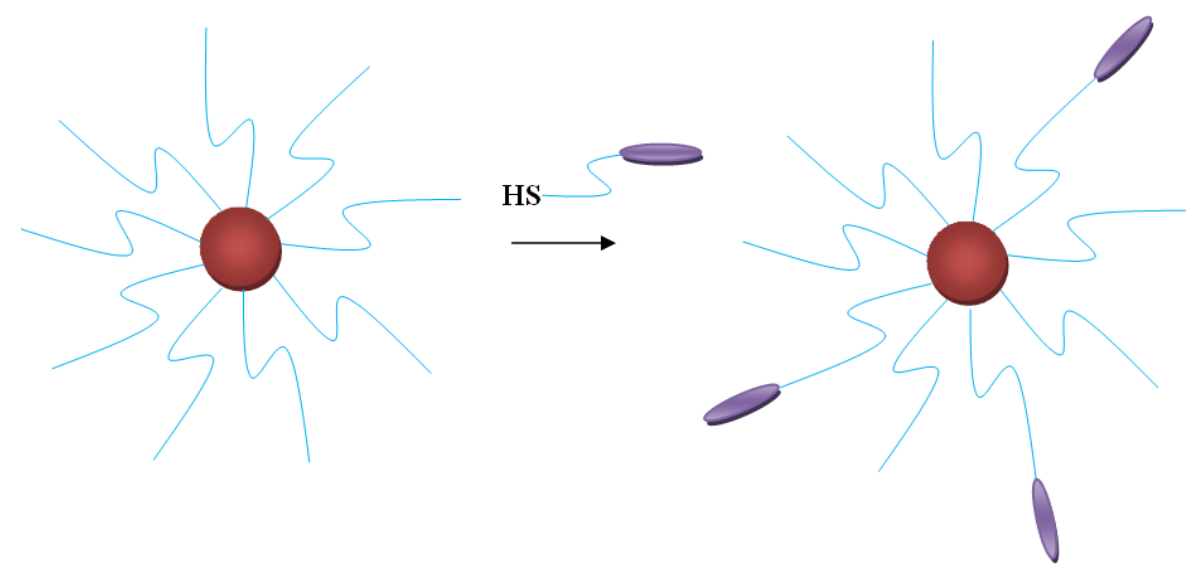

Figure 11: Modification of MPCs by the Murray place exchange reaction to yield MMPCs.

Despite the numerous advantages offered by the Brust method, NPs synthesised by this technique are unsuitable for use in biological applications. This is due to the synthesis being carried out in organic solvents, resulting in GNPs that inherently lack the necessary stability and solubility in aqueous media, a fundamental requirement in biological applications. ${ }^{34-36}$ As a result, an alternate technique should be employed to accommodate the biological requirements of the resultant GNPs.

The NP synthesis can be carried out using the $\mathrm{NaBH}_{4}$ reduction, in which the GNPs can be synthesised in an aqueous environment. 


\subsubsection{Sodium borohydride method}

The reduction of $\mathrm{HAuCl}_{4}$ by the $\mathrm{NaBH}_{4}$ reduction method is a simple process: An aqueous solution of $\mathrm{NaBH}_{4}$ is added as the reducing agent in the presence of thiolate ligands (Figure 12) to an aqueous solution of $\mathrm{HAuCl}_{4}{ }^{33}$

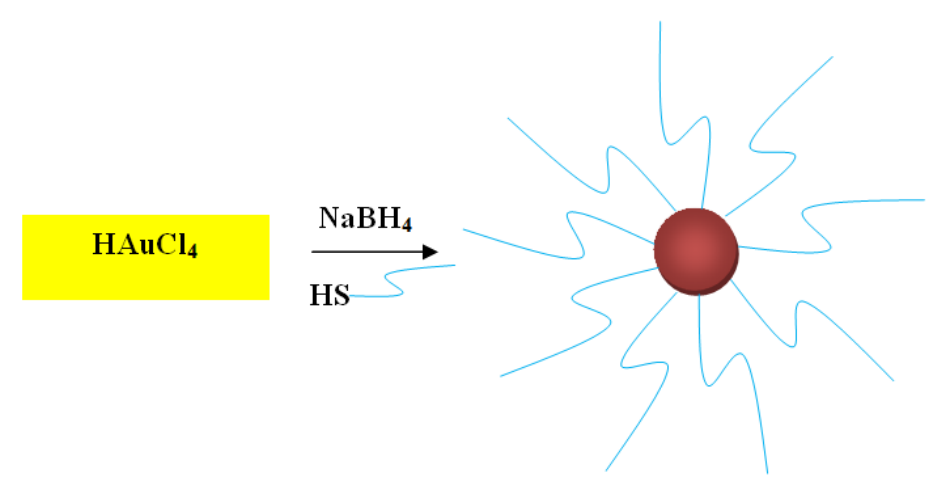

Figure 12: Construction of thiol stabilised MPCs through the $\mathrm{NaBH}_{4}$ reduction method.

As in the citrate method, the particle size can be varied by adjusting the concentration of reducing agent to gold salt - the larger the ratio, the smaller the particle size. ${ }^{\mathbf{3 0 , 3 1}}$ The ligands control solubility and prevent aggregation of the resulting NPs. The reduction by $\mathrm{NaBH}_{4}$ is simple, rapid and does not require the use of phase transfer agents such as TOAB that is used in the Brust method. The reduction can be performed at room temperature, unlike the citrate reduction procedure which requires refluxing and ultraclean glassware. This method is also advantageous as the synthesis is carried out in one easy step. As the GNPs are synthesised in an aqueous environment, the resultant GNPs are suitable for the use in biological applications.

\subsection{OPTICAL PROPERTIES OF GNPS}

GNPs are known for their beautiful cherry red colour in solution as well as their extremely high molar absorbtivity. ${ }^{37}$ The strong light absorbance of GNPs is ascribed to the transverse oscillations of the conduction electrons at the surface of the NPs in response to the alternating electromagnetic field of the incoming light which induces a dipole moment. ${ }^{\mathbf{3 0 , 2 9 , 3 8}}$ This phenomenon is known as plasmon resonance and is depicted in Figure 13. 
(A)
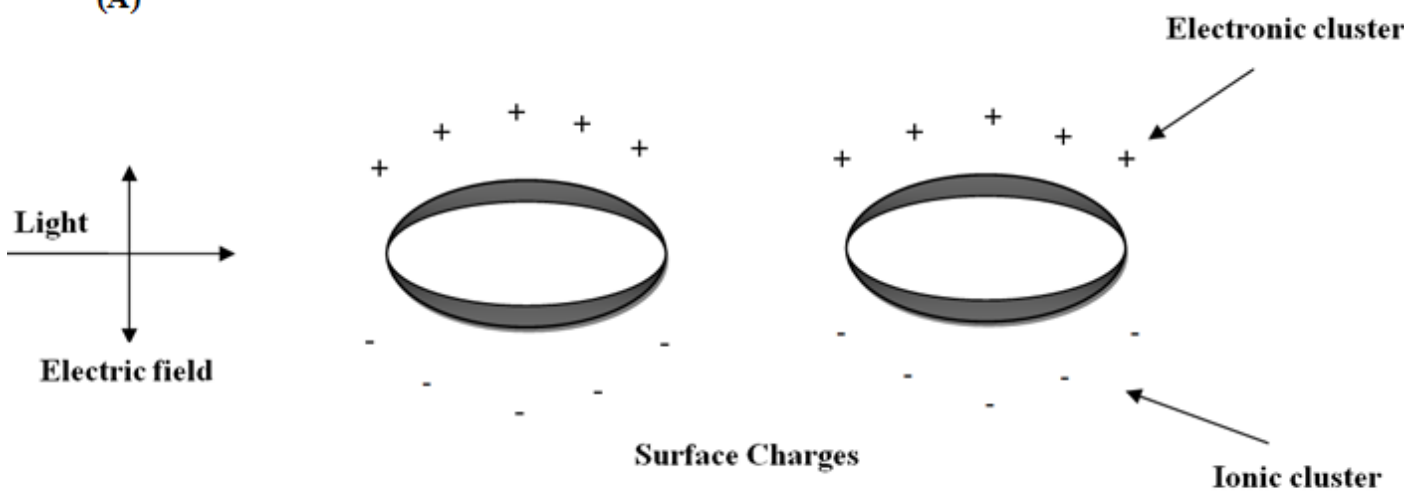

(B)

Surface Charges

Ionic cluster
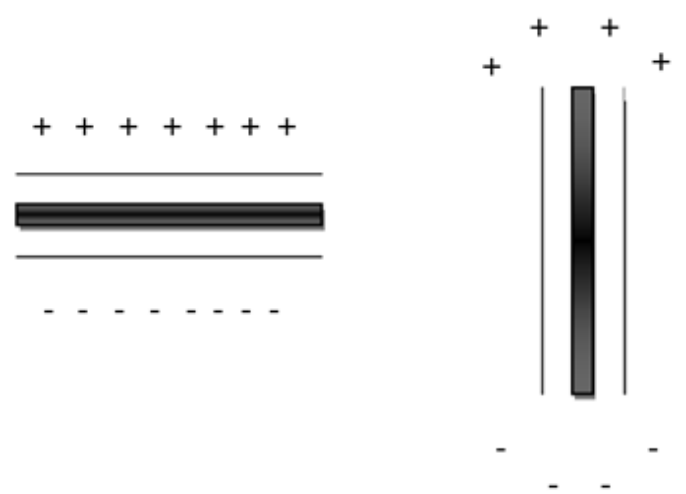

Figure 13: Schematic drawing of the principle of plasmon resonance. A dipole is induced (A), which oscillates in phase with the electric field of the incoming light (B). Redrawn from Huang (2006). ${ }^{39}$

The maximum absorbance wavelength of the surface plasmon band of GNPs is around $520 \mathrm{~nm}$ and is responsible for the brilliant colour of the NP in solution (Figure 14). ${ }^{\mathbf{2 9}}$ 


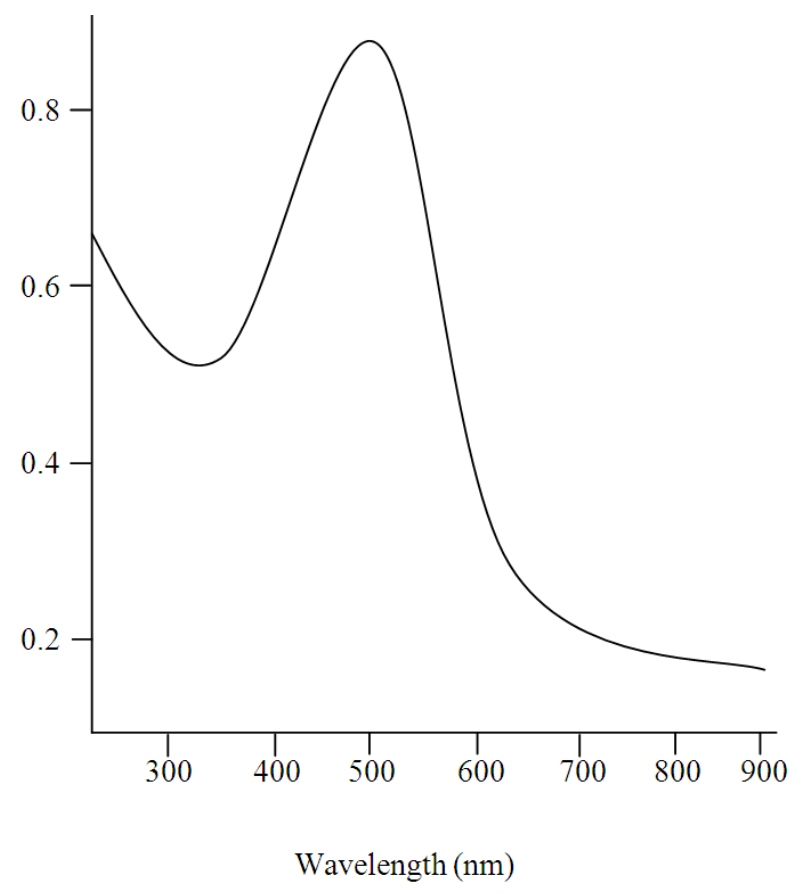

Figure 14: Typical surface plasmon absorption spectrum of spherical GNPs. Redrawn from Huang (2006). ${ }^{39}$

As mentioned previously, the surface plasmon resonance band is dependent on the size of the NPs. ${ }^{37}$ An increase in size results in a red shift of the absorbance maximum of the surface plasmon resonance band to longer wavelengths $(518$ to $533 \mathrm{~nm}$ ). The bandwidth is also affected by the NPs size - the bandwidth decreases with increasing size when the NPs are less than $20 \mathrm{~nm}$ and increases with increasing NP size when the NPs are greater than $20 \mathrm{~nm}$. Inter-particle distance also has an effect on the surface plasmon band of NPs. ${ }^{37}$ In the aggregated state, the individual NPs are able to interact with each other to give rise to oscillations that are the combination of oscillations of the individual NPs causing a red shift in the band upon aggregation. These unique optical features make GNPs attractive probes for biosensing, molecular imaging and allow for use in many photothermal therapy applications. ${ }^{\mathbf{4 0}}$ 


\subsection{CHARACTERIZATION OF GNPS}

Many techniques are currently available for the characterization of GNPs. These techniques include transmission electron microscopy (TEM), atomic force microscopy (AFM), scanning tunnelling microscopy (STM), Fourier transform infrared spectroscopy (FTIR), nuclear magnetic resonance (NMR), and ultraviolet-visible spectroscopy (UV-vis). The core of the GNP can be viewed via TEM and information such as the size of the core, the disparity of the NP population, and the shape of the NPs formed can be obtained. AFM and STM can be used to elucidate the size of the NPs formed and unlike TEM the dimensions generally include the molecules in the ligand shell. FTIR, NMR and UV-vis are used to verify the structure of the ligand shell as well as qualitatively measure purity since the peaks of free alkanethiols are narrower than those on the NP itself.

\subsection{BIOLOGICAL APPLICATIONS OF GNPS}

NPs are comparable in size to many common biomolecules and are therefore natural companions in hybrid systems. ${ }^{\mathbf{4 1}}$ This proposal of merging biological and non-biological application systems at the nanoscale level has proven useful for research in cell and molecular biology. NPs and biological molecules have been combined to exploit the many attributes of the NPs and the specific molecular recognition capabilities of the biological molecules in biotechnological applications. ${ }^{16,38,42-44}$

To be viable for the use in disease diagnostics and therapy, it is essential that the NP systems are functionalised for the following reasons:

- Recognition of specific target sites in the body after systematic administration,

- to impart good biocompatibility,

- to ensure enhanced solubility. ${ }^{45,46,42}$

This surface functionalisation of GNPs is of utmost importance in biomedical applications as it facilitates a selective and effective means of controlled intracellular release. ${ }^{45}$ The gold core imparts the necessary stability to the manufactured assembly while the surrounding thin monolayer allows tuning of surface properties such as charge, optical properties, and hydrophobicity. The monolayer is largely associated with chemical behaviour of the GNPs such as solubility, molecular recognition and organization. In a typical assembly, hydrophobic groups are located in the interior part of the macromolecule to provide a tightly packed protective shield to the NPs, while charged and hydrophilic molecules are exposed to the solution, ensuring solubility in water. ${ }^{\mathbf{3 4 , 4 6 , 4 7}}$ 
GNPs can be attached to biomolecules such as proteins, peptides, viruses, carbohydrates, antibodies, lipids and nucleic acids. ${ }^{48}$ There has been substantial interest in the interaction between peptides and GNPs to utilise the improved bio-specificity of the resulting GNPs in order to expand applications of these systems in biological and medical sciences. ${ }^{49}$

These peptide-derivatised NPs are becoming increasingly popular due to their ease of synthesis and ability to alter the properties of the macromolecule. They make use of cysteine as an anchor group to stabilise the GNPs: Cysteine-terminated peptides are covalently coupled directly to the gold particles surface through a sulfur-gold bond. ${ }^{\mathbf{5 0}}$ The choice of peptide sequence and length determines the solubility of the macromolecule as well as cell permeability of the resulting GNP composite. The functionalisation of GNPs with hydrophillic amino acid residues such as lysine and arginine induces a positive charge on the NP that favours interaction with the membrane (charged negatively) and consequently enhances penetration into the cell. There are several examples in the literature describing the addition of lysine or arginine for transfecting molecules into cells. ${ }^{\mathbf{1 1}}$

Peptides were coupled to GNPs in this study due to the myriad of advantages they afford to the GNP composites. The chemistry of peptides is discussed in Chapter 3. 


\section{CHAPTER 3: PEPTIDE CHEMISTRY}

Peptides are essential in the design strategy of this project and are hence discussed in detail in this section.

\subsection{A GENERAL INTRODUCTION}

Amino acids are the building blocks for the assembly of peptides and proteins. Every living organism on earth uses mainly the same 20 amino acid residues (Table 1) and therefore amino acids are referred to as the building blocks of life. ${ }^{\mathbf{5 1}}$ They are characterised by two main functional groups, namely a carboxylic acid group and an amino group. Both groups are bonded to the same carbon atom which is referred to as the $\alpha$-carbon or $\mathrm{C} \alpha$ which is a chiral centre, except for glycine. ${ }^{\mathbf{5 2}}$ The $\mathrm{C} \alpha$ atom is substituted with a hydrogen atom and a side-chain group (R), except in the case of glycine for which $\mathrm{R}=\mathrm{H}^{\mathbf{5 3}}$

Side-chain groups are distinctive for each amino acid. The side chains determine the properties of peptides and contribute to the structure as well as the function of these biomacromolecules. ${ }^{\mathbf{5 2}}$ Amino acids are connected to each other in a linear sequence to form peptides/proteins. ${ }^{\mathbf{5 2}}$ Molecules consisting of 100 or more amino acid residues are regarded as proteins and those consisting of less than 100 residues are referred to as peptides. Molecules made up of 10-100 amino acid residues are called polypeptides. ${ }^{54}$ The covalent bond formed between two amino acids is known as an amide or peptide bond and is a result of condensation of the $\alpha$-carboxyl group of one amino acid with the $\alpha$-amino group of another (Figure 15). ${ }^{\mathbf{5 5 - 5 7}}$<smiles>[R]C(N)C(=O)O</smiles><smiles>[R]C(N)C(=O)O</smiles><smiles>CC=O</smiles><smiles>[R]C(N)C(=O)NC([R1])C(=O)O</smiles>

Figure 15: Reaction depicting peptide bond formation. ${ }^{\mathbf{5 4}}$ 
Table 1: Standard amino acids with their codes: The three-letter code is the abbreviated form of the full amino acid name and the one-letter code is a single letter representation of the full amino acid name. The one-letter code is used to donate peptide sequences. ${ }^{\mathbf{5 8}}$

\begin{tabular}{|c|c|c|}
\hline Amino acid & Three-letter code & One-letter code \\
\hline Alanine & Ala & A \\
\hline Arginine & Arg & $\mathrm{R}$ \\
\hline Asparagine & Asn & $\mathrm{N}$ \\
\hline Aspartic acid & Asp & $\mathrm{D}$ \\
\hline Cysteine & Cys & $\mathrm{C}$ \\
\hline Glutamic acid & Glu & $\mathrm{E}$ \\
\hline Glutamine & Gln & Q \\
\hline Glycine & Gly & G \\
\hline Histidine & His & $\mathrm{H}$ \\
\hline Isoleucine & Ile & I \\
\hline Leucine & Leu & $\mathrm{L}$ \\
\hline Lysine & Lys & K \\
\hline Methionine & Met & M \\
\hline Phenylalanine & Phe & $\mathrm{F}$ \\
\hline Proline & Pro & $\mathrm{P}$ \\
\hline Serine & Ser & $\mathrm{S}$ \\
\hline Threonine & Thr & $\mathrm{T}$ \\
\hline Typtophan & $\operatorname{Trp}$ & $\mathrm{W}$ \\
\hline Tyrosine & Tyr & $\mathrm{Y}$ \\
\hline Valine & Val & $\mathrm{V}$ \\
\hline
\end{tabular}

The free amino group and free carboxyl group on opposite sides of the peptide chain are referred to as $N$-terminus and $C$-terminus respectively. Amino acid residues in a peptide chain are conventionally written from the $N$-terminus to the $C$-terminus. ${ }^{\mathbf{5 9 , 5 4}}$ 


\subsection{PEPTIDE SYNTHESIS}

Two techniques can be used for the synthesis of peptides: Solution- and solid-phase synthesis, the latter is abbreviated as SPPS. ${ }^{\mathbf{5 4}}$ The former technique is the classical approach to peptide synthesis but is nowadays seldom used whereas the latter is used by most laboratories worldwide and has several advantages over the former. The advantages of SPPS are:

- Ease of purification between steps,

- possibility of full automation,

- synthetic intermediates do not have to be isolated,

- excess reagents can be used in order to drive difficult reactions to completion,

- overall a quicker method of peptide synthesis. ${ }^{59-61}$

\subsubsection{Solid phase peptide synthesis}

SPPS was pioneered by Merrifield in 1963 and was initially based on the use of tert-butoxycarbonyl (Boc) $\alpha$-amino protecting group. The original Merrifield method often suffers from two limitations:

- The Boc protecting group is often removed by treatment of the resin with trifluoroacetic acid (TFA), thus only acid stable protecting groups can be used for side chain protection,

- hydrogen fluoride (HF) treatment is required for cleavage of the peptide from the resin and is often the cause of unwanted by-products. ${ }^{\mathbf{6 1}}$

Due to the disadvantages of the Merrifield method, Carpino and Han (1972), introduced the use of the base labile $\alpha$-amino protecting agent, namely the $N$-fluorenylmethyloxycarbonyl (Fmoc) group. ${ }^{62,63}$ The main advantage of the Fmoc strategy, over the formerly used Boc method, is due to the requirements of mild chemical conditions: The Fmoc group is readily removed in weak basic conditions such as $20 \% \mathrm{v} / \mathrm{v}$ piperidine in DMF. Most side chain protecting groups are unaffected under such conditions. ${ }^{\mathbf{5 4}}$ The conditions are also weak enough to prevent racemisation of the stereogenic centre ( $\alpha$-carbon). ${ }^{\mathbf{5 4}}$ Due to the obvious advantages of Fmoc chemistry, it will be discussed in detail in the following sub-sections. 


\subsubsection{Solid support and linkers}

The peptide chains are built on small resin beads which are insoluble in solution yet remain porous. ${ }^{60}$ The resin is a polymeric backbone to which the substrate is anchored. ${ }^{\mathbf{5 4}}$ The resin used for SPPS is a copolymer that has unique physical and chemical properties and is vital in the success of the SPPS method. It is essential that the resin beads swell in organic solvents to allow access to groups within the pores of the resin by chemical reagents during cycles of deprotection, coupling and washing steps. ${ }^{64}$

\subsubsection{Side chain protecting strategies}

Many of the amino acids used in the assembly of peptide chains have side chains that contain reactive functional groups. ${ }^{44}$ It is necessary to protect these reactive functionalities which are not directly involved in peptide bond formation. ${ }^{\mathbf{6 3 , 5 2 , 5 3}}$ Certain groups are temporarily protected whereas others are kept permanently protected until the end of the synthesis. The removal of permanent protecting groups generally occurs with the release of the peptide chain from the solid support. The following considerations are essential when selecting the correct temporary side chain protecting groups:

- Side chain protecting groups must be stable during coupling and deprotection steps (orthogonal chemistry),

- side chain protecting groups must be easy to remove, preferably during the cleavage step,

- the temporary protected groups must be readily removed under conditions that will not affect the permanent protecting groups ,

- removal of side chain protecting groups must not affect the newly synthesised peptide bond. ${ }^{63,52}$

A list of all the amino acids and their commonly used protecting groups are listed in Table 2. 
Table 2: The most common side-chain protecting groups for amino acids. ${ }^{\mathbf{5 8}}$

\begin{tabular}{|c|c|c|}
\hline Amino Acid & Protecting group & Functionality Protected \\
\hline Alanine & None & \\
\hline Arginine & Pbf, Mtr, Pmc & Guanidino N \\
\hline Asparagine & Trt, Mbh, Tmob & Amide \\
\hline Aspartic acid & otBu, OAll & Carboxyl \\
\hline Cysteine & Trt, Acm, $t \mathrm{Bu}, \mathrm{StBu}$ & Sulfhydryl \\
\hline Glutamine & Trt, Mbh, Tmob & Amide \\
\hline Glutamic acid & otBu, OAll & Carboxyl \\
\hline Glycine & n.a & \\
\hline Histidine & Trt, Boc & Imidazole \\
\hline Isoleucine & None & \\
\hline Leucine & None & \\
\hline Lysine & Boc, Aloc, Fmoc & Amino \\
\hline Methionine & None & \\
\hline Phenylalanine & None & \\
\hline Proline & None & \\
\hline Serine & $t \mathrm{Bu}$ & Hydroxyl \\
\hline Theonine & $t \mathrm{Bu}$ & Hydroxyl \\
\hline Tryptophan & Boc & Indole \\
\hline Tyrosine & $t \mathrm{Bu}$ & Phenol \\
\hline Valine & None & \\
\hline
\end{tabular}

\subsubsection{Fmoc deprotection}

The initial step in the removal of the $N$-Fmoc group is the deprotection of the fluorine ring to yield the aromatic cyclopentadiene type intermediate that rapidly rearranges to form dibenzofulvene. The latter is then scavenged by excess piperidine to afford the fulvene adduct (Figure 16). 

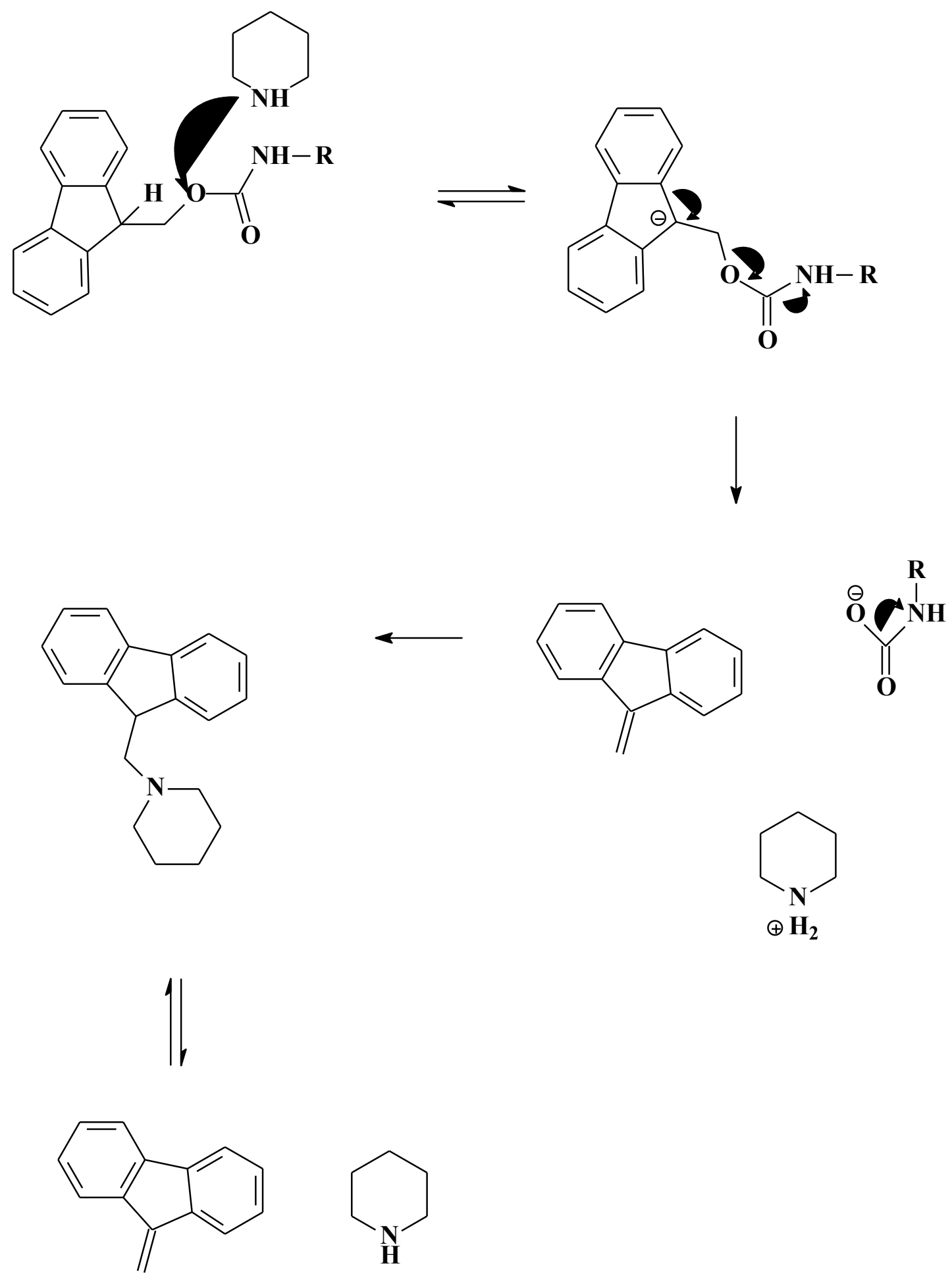<smiles>C1CCNCC1</smiles>

Figure 16: Mechanism of Fmoc cleavage redrawn from Sewald and Jakubke. ${ }^{\mathbf{5 4}}$

Once deprotection of the $\mathrm{N}$-Fmoc group is complete it is necessary to form a new peptide bond. This step requires the activation of the $\alpha$-carboxyl group of an amino acid so as to react with the free $\alpha$-amino group of another amino acid, already attached to the peptide resin. 


\subsubsection{Activating strategies}

To facilitate the formation of the amine bond between the amine group of the growing peptide and the carboxylic group of the incoming Fmoc-protected amino acid, activating groups (also known as coupling reagents) are used to activate the carboxylic group (Figure 17). ${ }^{\mathbf{5 5 , 6 3 , 5 2 , 5 4}}$<smiles>[R]C(=O)NC(C)C(=O)O</smiles><smiles>[R]NC(=O)C(C)N</smiles><smiles>[X]OC(=O)C(C)NC([R])=O</smiles>

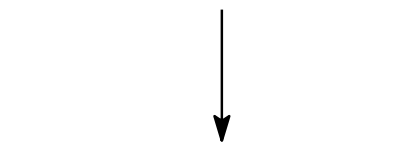<smiles>[R]NC(=O)C(C)NC(=O)C(C)NC([R])=O</smiles>

Figure 17: Mechanism of peptide bond activation redrawn from Bodansky. ${ }^{65}$

Numerous coupling reagents are currently available including carbodiimides, acyl hydrides, anhydrides, acyl halides, active esters and uranium and aminium reagents. ${ }^{\mathbf{5 4}}$

Carbodiimides were the first coupling reagents to be produced and were developed in 1955 by Sheehan and Hess. ${ }^{\mathbf{6 6}}$ The most popular of the carbodiimide-type coupling reagents in use today are dicyclohexylcarbodiimide (DCC) and diisopropylcarbodiimide (DIC). The carbodiimides play a dual role in the peptide coupling reaction, namely they serve to form an activated ester with the incoming amino acid, and react with the water released during the "condensation" reaction to form the corresponding urea. DCC is less suitable for SPPS since the corresponding urea that is formed is insoluble in most organic solvents. DIC forms a soluble urea which is washed away with ease during SPPS. Some of the advantages of the carbodiimides are that they are inexpensive and can allow for rapid coupling. ${ }^{\mathbf{5 2 , 5 3}}$ The main problem associated with the use of carbodiimides is their high reactivity that can lead to racemisation of the amino acid. The problem of racemisation can be 
suppressed by the addition of aromatic oximes such as 1-hydroxy-benzotriazole (HOBT), proposed by Konig and Geiger in $1970 .{ }^{\mathbf{6 5 , 6 2}}$

More recently uranium complexes such as 2-(1H-7-Azabenzotriazol-1-yl)-1,1,3,3-tetramethyl uronium hexafluorophosphate methanaminium (HATU) and O-Benzotriazole- $\mathrm{N}, \mathrm{N}, \mathrm{N}^{\mathrm{ee}}, \mathrm{N}^{\mathrm{e}}$ tetramethyl-uronium-hexafluoro-phosphate (HBTU) were developed. These coupling reagents are far superior to both the carbodiimides and aromatic oximes and have thus gained wide use in effecting peptide bond formation, particularly in SPPS. ${ }^{\mathbf{5 9 , 6 0}}$

\subsubsection{Final cleavage}

In the final step of the synthesis, the peptide is cleaved from the resin together with side chain protecting groups. An extremely delicate balance is necessary between conditions that are sufficiently vigorous to remove the peptide from the resin and at the same time, are sufficiently mild to ensure the survival of sensitive structural features of the peptide. ${ }^{67}$

Concentrated TFA is commonly used for the simultaneous cleavage of the peptide from the resin and removal of side-chain protecting groups. ${ }^{68}$ During such cleavage conditions, the side-chain protecting groups produce stabilised carbocations which easily react with electron rich side-chain of some amino acids (cysteine, methionine, tyrosine, threonine, serine, tryptophan) resulting in the formation of unwanted by-products. This phenomenon is minimised by the addition of scavengers to the cleavage cocktail to effectively neutralize the carbocations. ${ }^{69}$ A wide variety of cleavage cocktails have been developed with the intention of optimising the conditions for particular peptide sequences. $^{70}$

\subsubsection{Automation and microwave synthesis}

A significant disadvantage of manual peptide synthesis is the amount of time and effort required in the attachment of individual molecules. ${ }^{\mathbf{5 8}}$ As a consequence, emphasis has been placed on the use of automation to allow for fast, economical and reliable production of a large number of peptides. SPPS is highly amenable to automation due to the repetitive nature of the key steps and the reliability of the chemistry. ${ }^{\mathbf{5 8}}$ Further rate enhancement of the time consuming SPPS technique has been reported by the application of microwave irradiation: Microwave enhanced peptide synthesis is reported to be 10-20 times faster than conventional SPPS, produces cleaner peptides with higher yields and lessens the possibility of chain aggregation. ${ }^{69}$ It is due to the plethora of advantages that 
manual SPPS has been widely replaced with automated sequential high-speed microwave chemistry. ${ }^{69}$ A graphical representation of the SPPS protocol is shown in Figure 18.<smiles>[Y][R1]C(NC(F)F)C(=O)O</smiles>

$$
\begin{gathered}
\text { Activation } \\
\text { (HBTU) }
\end{gathered}
$$<smiles></smiles>

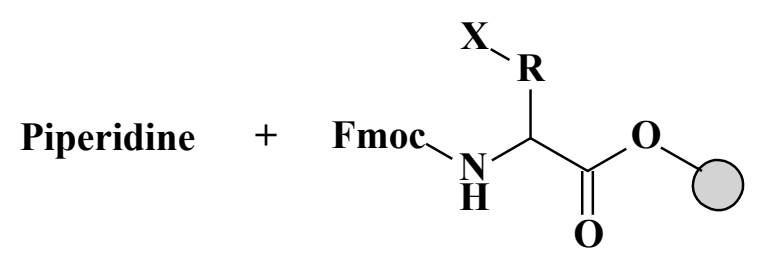
(Piperidine)<smiles>[R]C(N)C(=O)O[O-]</smiles>

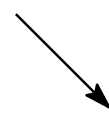

\section{Coupling}

(Basic pH)

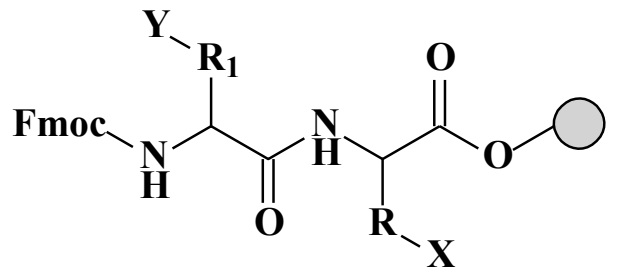<smiles>[R]C(NN)C(=O)NC([Y])C(=O)OOCO</smiles><smiles>[R]C(NN)C(=O)NC([R])C(=O)O[CH]</smiles>

Figure 18: Principle of SPPS redrawn from Sigma-Aldrich: Custom peptide synthesis. ${ }^{71}$ 
In this study, it was necessary to combine the properties of peptides and GNPs (section 2.6., page 22) in order to achieve the envisaged prodrug system. The optimisation of the peptide and GNP moieties required to obtain the ideal peptide-GNP system will be discussed in Chapter 4 and will also reveal the results of the peptide and GNP syntheses. 


\section{CHAPTER 4: ASSEMBLING OF PEPTIDES TO GNPS}

As mentioned in section 2.6. (page 22), GNPs and biological molecules have been combined to take advantage of their many favourable properties. In these systems it is essential that characteristics of both moieties are optimised for use in the desired application.

\subsection{DESIGN CONSIDERATIONS}

Currently, the ability to control and modify the properties of GNPs to better suit their integration within biological systems (such as controlling their size and modifying their surface layer for enhanced aqueous solubility, biocompatibility and biorecognition) is well established. ${ }^{\mathbf{3 8}}$ Certain considerations are essential to facilitate the use of GNPs in the required applications and are discussed in this section.

\subsubsection{GNP size considerations}

The circulation of particles in vivo has been shown to be size dependent as discussed by Tracy et al. $(2007)^{72,73}$ and it is therefore essential to strictly control the size of the particles. The following considerations should be taken into account in selection of the ideal NP size:

- Particles less than two $\mathrm{nm}$ in diameter do not show the characteristic plasmon resonance band and cannot be observed by light microscopy,

- only particles less than $30 \mathrm{~nm}$ in diameter are able to traverse pores,

- access to tumour tissue from the vasculature is limited to particles less than $70 \mathrm{~nm}$ in diameter,

- to allow for efficient cell uptake the NPs should not exceed $200 \mathrm{~nm}$ in diameter. ${ }^{\mathbf{7 2 , 7 4}}$

It can thus be concluded that particles with a diameter size of 10-30 nm are desirable. Size-control can be achieved by varying the Au:thiol ratio, with smaller particles being formed when a greater amount of thiol is used. 


\subsubsection{GNP synthesis considerations}

As discussed in section 2.3. (page 16), there are three common methods used for the synthesis of GNPs, namely the sodium citrate or Turkevitch method, the Brust method and the $\mathrm{NaBH}_{4}$ reduction. The only viable methods for use in this particular project are either the sodium citrate or the $\mathrm{NaBH}_{4}$ method as GNPs can be synthesised in aqueous media. The method of choice for the synthesis of GNPs in this study was the sodium citrate reduction due to the following two reasons:

- GNPs synthesised using the sodium citrate method are generally $15 \mathrm{~nm}$ in size, an appropriate size for this study,

- the sodium citrate method generates GNP solutions that can remain stable for several months due to the strong electrostatic repulsion generated by the negative sodium citrate ions, the stability of which can be significantly enhanced by the covalent attachment of thiolated ligands. $^{25}$

\subsubsection{Monolayer considerations}

It is necessary to tailor the GNPs to specifically suit the final biomedical application. As a result, a great deal of consideration must be given to the selection of the groups attached to the GNP surface. Peptides are ideal molecules for attachment to GNPs as they facilitate the stabilization of the GNPs and can easily be modified to be suitable in biological systems. Both peptide sequence and length must be carefully selected to ensure that the desirable properties of the macromolecule are obtained.

\subsubsection{The sequence and length of peptide attached to GNPs}

As mentioned previously (section 2.6., page 22), a successful GNP assembly consists of hydrophobic spacer groups on the interior and hydrophilic molecules on the exterior part of the macromolecule, ensuring the solubility and stability of the molecule. PolyAlanine (polyA), a hydrophobic peptide sequence, can be directly attached to the GNP surface with a cysteine residue. The exterior section of the macromolecule can consist of the more hydrophilic sequence K-DAFKRK where the tripeptide D-AKF is the tumour-specific sequence that is selectively recognised by the tumour-associated protease, plasmin. ${ }^{5}$

As discussed in section 1.3. (page 5), fluorescence was utilised as a tool to prove the experimental concept and was provided by the fluorophore, Coumarin-3-carboxylic acid. It is well known that fluorophores in close proximity to GNPs fluoresce weakly due to a phenomenon known as fluorescence resonance energy transfer (FRET) (refer to section 5.1., page 65, for details). The 
length of the peptide sequence should be sufficiently long in order to avoid the occurrence of FRET between Coumarin-3-carboxylic acid and the gold centre. According to Aslan et al. (2007), ${ }^{\mathbf{7 5}}$ a strong quenching of fluorescence emission and a dramatic reduction of the lifetimes of the excited states is observed when the separation distance between fluorophores and metallic surfaces is less than $5 \mathrm{~nm}$. Distances in the range of $10-20 \mathrm{~nm}$ can result in enhanced fluorescence emission due to local concentration of the incident excitation field by the metallic NPs. ${ }^{\mathbf{7 5}}$ Cys-Ala $_{35}$ is approximately $10 \mathrm{~nm}$ in length and should be sufficiently long to prevent FRET from occurring (refer to section 4.2.2., page 37, for results).

The peptide sequence and length also becomes crucial when the issue of stability of the peptideGNP composite is examined: Storhoff et al. ${ }^{\mathbf{7 6}}$ noticed an enhancement of GNP stability with an increase in oligonucleotide length. As a result, the stability of the peptide-GNP composites as a function of peptide length will be investigated (section 4.3.9., page 60).

\subsection{RESULTS AND DISCUSSION OF PEPTIDE SYNTHESIS}

The synthesis of peptides and ligands attached to GNPs is described in the following section. Peptide sequences are identified using single letter notation for the constituent amino acids (refer to Table 2 for amino acid notation).

\subsubsection{Synthetic difficulties encountered with cysteine containing peptides}

Cysteine is unstable, highly reactive in solution and readily undergoes oxidation of its thiol group with intra- or inter-chain disulfide bridge formation to form the dimer, cystine. The formation of the dimer is undesirable as it would hinder the covalent attachment of the thiol group to the gold surface. This can be prevented by the protection of the highly reactive thiol group of the terminal cysteine throughout the peptide synthesis steps (until the peptide is cleaved from the resin). Tertiary butyl $(t \mathrm{Bu})$ is the ideal protecting group for this purpose as it is stable to TFA (used in the cleavage mixture). The $t \mathrm{Bu}$ group can then be removed prior to lyophilisation of the peptide.

The $t \mathrm{Bu}$ protecting group, however, requires additional procedures for deprotection after cleavage of the peptide from the resin. This can be achieved as this group is labile towards mercury(II) salts. $^{\mathbf{5 4 , 7 7 , 7 8}}$ The deprotection procedure [as described by Applied Biosystems (2008) ${ }^{\mathbf{7 9}}$ and McCurdy et al. $(1989)^{\mathbf{7 8}}$ ] was attempted several times on the CKAFKRK peptide (section 4.2.4., 
page 39) with little success. Despite sources mentioning the use of this procedure, there are other reports that dispute the effectiveness of the deprotection step. ${ }^{\mathbf{8 0}}$

According to a personal communication with Professor Fernando Albericio ${ }^{\mathrm{g}}$, the free sulfhydryl group should not pose a severe problem, provided that the peptide does not remain in solution for long periods of time and the solution is not heated to temperatures above $50^{\circ} \mathrm{C}$. As a result, it was decided to use the Cys(trityl) (Trt) protecting group that is easily removed upon TFA deprotection. The constraints mentioned by Professor Fernando Albericio were followed and the peptide was successfully synthesised without the formation of any unwanted dimers. The products were lyophilised and stored as powders in order to prevent oxidation or degradation of the peptides.

\subsubsection{Cysteine-(alanine) $)_{35}$}

It is a well known fact in the peptide community that it is extremely difficult to achieve adequate yields of very hydrophobic peptides. These peptide sequences are difficult to synthesise and pose numerous difficulties during cleavage and purification processes. In most of the established hydrophibicity scales, alanine is considered moderately apolar and thus falls into the category of a „difficult peptide sequence ${ }^{e e}$. The hydrophobicity of polyA leads to severe aggregation that is primarily the result of the self-association of polymer-bound peptide sequences by intermolecular hydrogen bonding as depicted in Figure 19. ${ }^{\mathbf{8 1}}$ This phenomenon is caused by the formation of intermolecular $\beta$-sheet aggregates that are responsible for lowering coupling efficiencies. ${ }^{\mathbf{5 8}}$

\footnotetext{
g The University of KwaZulu-Natal, Westville campus chemistry department has a research collaboration with Professor Fernado Albericio 's research group in Barcelona, Spain.
} 


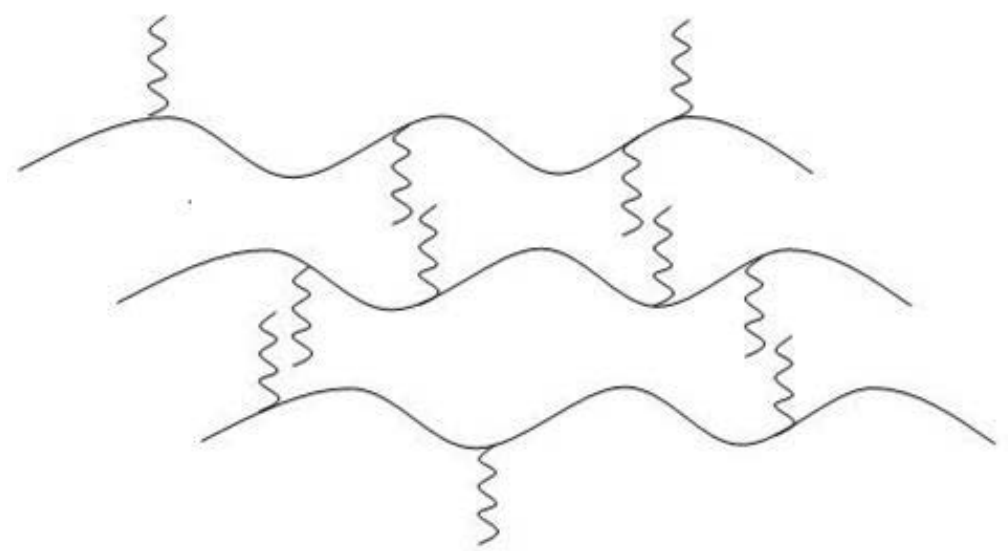

Figure 19: Scenario depicting the solvation or aggregation state of polymer-bound polyA sequence. The peptide chains are inter-molecularly aggregated whilst the polymer is solvated effectively increasing the overall level of cross-linking in the matrix. Redrawn from Chan et al. (2000). ${ }^{\mathbf{5 8}}$

Despite these synthetic difficulties, Bechinger (2001) ${ }^{\mathbf{8 2}}$ managed to synthesise polyA peptides with relatively high purity. Due to Bechinger's successes in the synthesis of polyA peptides, the synthesis of cysteine-(alanine $)_{35}\left(\mathrm{CA}_{35}\right)$ was attempted. Subsequent analysis of the resultant peptide indicated that the crude peptide was impure. The gel-like nature of the peptide made solubility impossible, rendering purification unfeasible. Since the aggregation of polyA was too severe, it was decided to synthesise a sequence containing fewer alanine residues.

\subsubsection{Cysteine-(alanine) $)_{4}$-(glycine $)_{5}$}

Glycine, the simplest amino acid present in proteins, lacks the presence of a side chain and is thus able to destabilise $\beta$-sheets. ${ }^{\mathbf{8 3}}$ Chan et al. (2000) ${ }^{\mathbf{5 8}}$ reported that the minimum length at which polyA exhibits self-aggregation is five alanine residues. It was thus decided to incorporate glycine residues into the polyA sequence in order to reduce the aggregation potential of the peptide and thus cysteine-(alanine $)_{4}$-(glycine $)_{5}\left(\mathrm{CA}_{4} \mathrm{G}_{5}\right)$ sequence was decided upon. Surprisingly, the purity of the crude peptide was very low and the peptide was as insoluble in most solvents. A rationale was obtained for this result from the work of Warrass et al. (2000): ${ }^{\mathbf{8 4}}$ The incorporation of glycine residues leads to the same aggregation potential as a peptide containing only alanine residues. 


\subsubsection{Cysteine (lysine- D-alanine-phenylalanine-lysine-arginine-lysine)}

Warrass et al. (2000) ${ }^{\mathbf{8 4}}$ mention that $\mathrm{D}$-Alanine acts as a $\beta$-sheet breaker and therefore the incorporation of D-Ala residues into an all-alanine sequence significantly reduces the aggregation potential of the sequence. The synthetic difficulties encountered were taken into account in the selection of the ideal peptide sequence for covalent attachment to the GNPs. Consequently the peptide sequence contained:

- Alternating D-AFK segments,

0 as the incorporation of $\mathrm{D}$-Alanine prevents the formation of $\beta$-sheet structures,

D-AFK is a tumour specific sequence,

- hydrophilic residues, such as lysine and arginine,

○ to ensure solubility,

- the functionalisation of GNPs with hydrophillic amino acid residues, such as lysine and arginine, induces a positive charge on the NP that favours interaction with the membrane (charged negatively) and consequently enhances penetration into the cell. There are several examples in the literature describing the addition of lysine or arginine for transfecting molecules into cells, ${ }^{\mathbf{1 1}}$

- hydrophobic D-Alanine and phenylalanine. These residues were expected to impart the necessary stability to the GNP macromolecule, thereby preventing aggregation.

The structures of the amino acids selected for peptide synthesis are shown in Table 3. Refer to Appendix 1 (page 138) for mass spectral characterization of the peptides and Table 4 for information about the synthesised peptides.

The overall sequence decided upon was Cysteine (lysine- D-alanine-phenylalanine-lysine-argininelysine $)_{\mathrm{n}}\left[\mathrm{C}(\mathrm{K}-\mathrm{D}-\mathrm{AFKRK})_{\mathrm{n}}\right]$. It was deemed necessary to gain ,hands on experience in the field of SPPS, thus manual synthesis of a peptide sequence was attempted. Due to the synthetic difficulties encountered with cysteine containing sequences, it was decided to synthesise a sequence with a terminal glycine as opposed to a terminal cysteine. The tumour specific sequence D-AFK is an essential component of the peptides utilized in this project and thus the sequence G- D-AFK was synthesized via manual peptide synthesis (refer to appendix 1, page 139, for mass spectral characterization).

CK-D-AFKRK, CK-D-AFKRKCoum and C(K-D-AFKRK $)_{3}, \mathrm{C}(\mathrm{K} \text {-D-AFKRK })_{3}$ Coum were then synthesised using automated SPPS. CK-D-AFKRK and CK-D-AFKRKCoum were successfully 
synthesised in high yield (94\% and 95\%) and purity (95\% and 97\%) and the observed mass corresponded with the expected masses of 880 and $1051 \mathrm{~m} / \mathrm{z}$. Refer to appendix 1 (page 140 and 141) for mass spectral characterization. This was not the case for the sequence Cysteine (lysine-Dalanine-phenylalanine-lysine-arginine-lysine $)_{3}\left[\mathrm{C}(\mathrm{KAFKRK})_{3}\right]$. The experimentally observed mass for $\mathrm{C}(\mathrm{KAFKRK})_{3}$ was $2526 \mathrm{~m} / \mathrm{z}$, a mass of $128 \mathrm{~m} / \mathrm{z}$ greater than the expected mass of $2398 \mathrm{~m} / \mathrm{z}$. This indicates that an additional lysine residue of mass $128 \mathrm{~m} / \mathrm{z}$ was coupled to the sequence. As the length and the ability to be cleaved are the main requirements of the peptide sequence, both of which remain unaltered by the addition of a lysine, this sequence was considered suitable for use in the required application. $\mathrm{C}(\mathrm{KAFKRK})_{3}$ was successfully synthesised in high yield $(87 \%)$ and purity (96\%). Refer to appendix 1 (page 142) for mass spectral characterization. $\mathrm{C}(\mathrm{KAFKRK})_{3}$ Coum was successfully synthesised in high yield (84\%) and purity (95\%) and the observed mass corresponded with the expected mass of $2571 \mathrm{~m} / \mathrm{z}$. Refer to appendix 1 (page 142) for mass spectral characterization. 
Table 3: Structures of the amino acids selected for peptide synthesis.

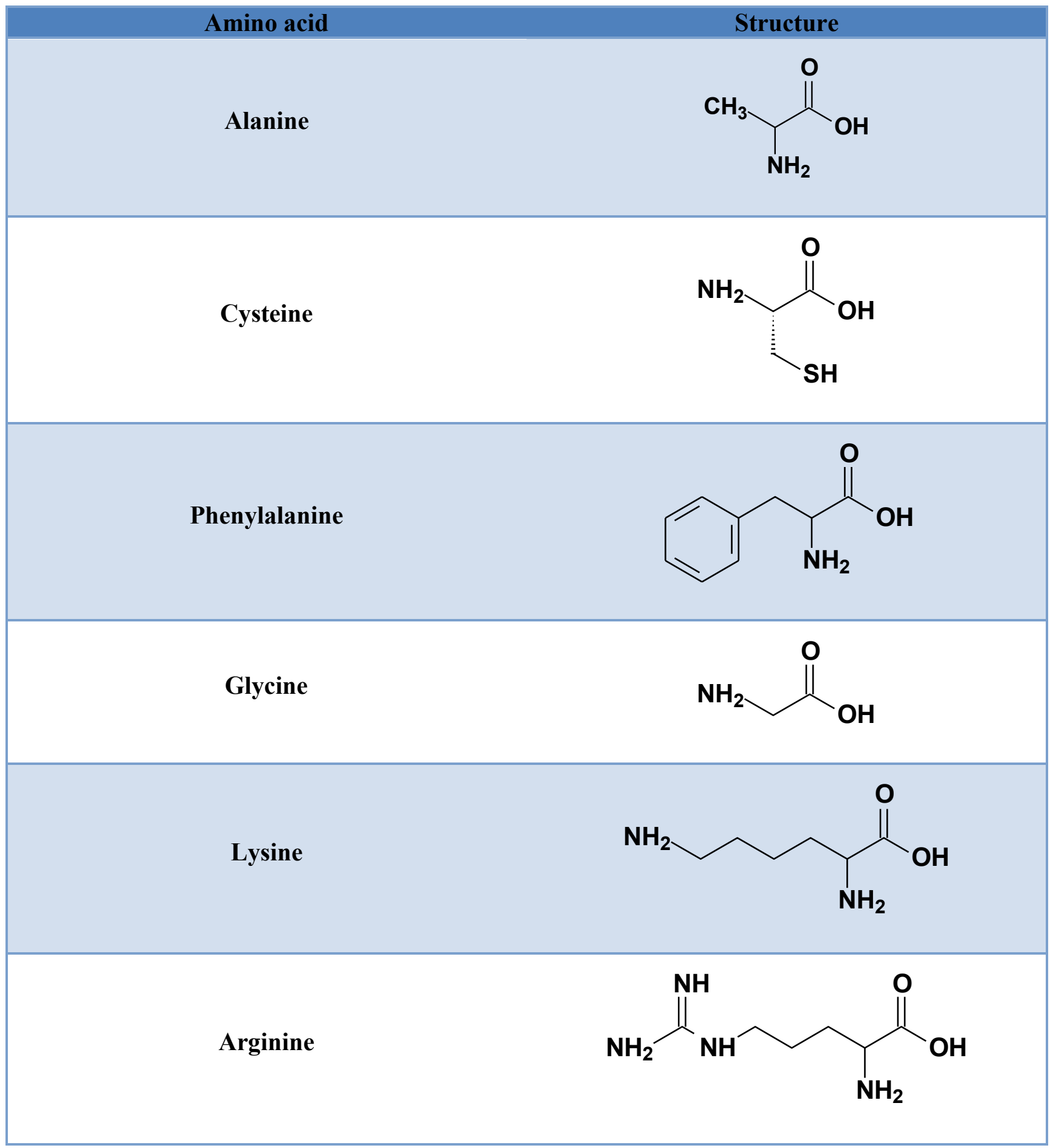

\subsubsection{Cysteine-glycine-D-alanine-phenylalanine-lysine-glycine-glycine-Coumarin}

The lysine and arginine residues in the sequence CK-D-AFKRKCoum were substituted with glycine residues in order to determine the effect of less hydrophilic residues on the ease of synthesis of the peptide as well as the effect on stability and solubility of the functionalised GNPs. The peptide was successfully synthesised with relatively good yield (85\%) and purity (96\%). Refer to Appendix 1 
for mass spectral characterization of the peptide and to Table 4 for information about the synthesised peptides and Appendix 1 (page 143) for mass spectral characterization.

\subsubsection{Cysteine-lysine-Coumarin}

The sequence cysteine-lysine-Coumarin (CKCoum) was synthesised in order to establish the effect of the peptide length on the solubility and stability of the GNPs. The peptide was successfully synthesised with high purity (98\%) and yield (90\%). Refer to Appendix 1 (page 144) for mass spectral characterization of the peptide and Table 4 for information about the synthesised peptides.

Table 4: The masses, yields and percentage purity of the successfully synthesised peptides.

\begin{tabular}{|c|c|c|c|c|}
\hline Peptide & $\begin{array}{c}\text { Expected } \\
\text { Mass }\left(\text { g.mol }{ }^{-1}\right)\end{array}$ & $\begin{array}{c}\text { Observed } \\
\text { Mass }\left(\mathrm{g} \cdot \mathrm{mol}^{-1}\right)\end{array}$ & Yield (\%) & Purity (\%) \\
\hline G-D-AFK & 419 & 420 & 93 & 97 \\
\hline CK-D-AFKRK & 880 & 880 & 94 & 95 \\
\hline $\mathrm{C}(\mathrm{K}-\mathrm{D}-\mathrm{AFKRK})_{3}$ & 2398 & 2526 & 87 & 96 \\
\hline CK-D-AFKRKCoum & 1051 & 1052 & 95 & 97 \\
\hline C(K-D-AFKRK $)_{3}$ Coum & 2571 & 2571.3 & 84 & 95 \\
\hline CG-D-AFKGGCoum & 810 & 810 & 85 & 96 \\
\hline CKCoum & 421 & 420.8 & 90 & 98 \\
\hline
\end{tabular}

\subsubsection{Cysteine methyl ester Coumarin}

According to Shang et al. (2007) ${ }^{\mathbf{8 5}}$ the addition of a single cysteine residue to the surface of GNPs significantly diminishes the effect of FRET. It was decided to test the validity of this observation. The ligand cysteine methyl ester Coumarin (CMECoum) was synthesised with the intention of capping the GNPs solely with CMECoum. Spectrofluorometric tests should indicate whether the fluorescence emission intensity of the Coumarin is diminished due to FRET. 


\subsection{RESULTS AND DISCUSSION OF GNP SYNTHESIS}

Two techniques were attempted for the synthesis of GNPs, namely the sodium citrate and the $\mathrm{NaBH}_{4}$ method.

\subsubsection{Sodium citrate}

The formation of the GNPs was confirmed by a colour change of the initial dispersion from pale yellow to wine red. This indicated the presence of spherical, monodispersed particles. The particles were characterised by TEM and UV-vis absorption spectroscopy. TEM images showed spherical and monodispersed particles and a mean diameter of $15 \mathrm{~nm}$ was calculated on the basis of the measurement of at least 200 particles (Figure 20) (refer to section 5.5., page 73, for details on TEM).

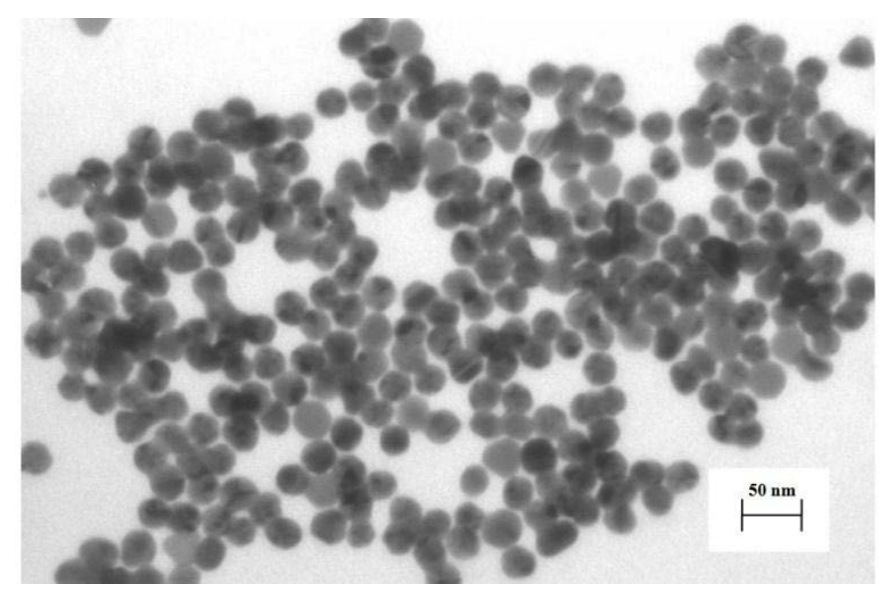

Figure 20: TEM image of citrate capped gold NPs in distilled water.

The formation of GNPs were verified by UV-vis absorption spectroscopy, wherein the characteristic plasmon bands are observed (Figure 21). 


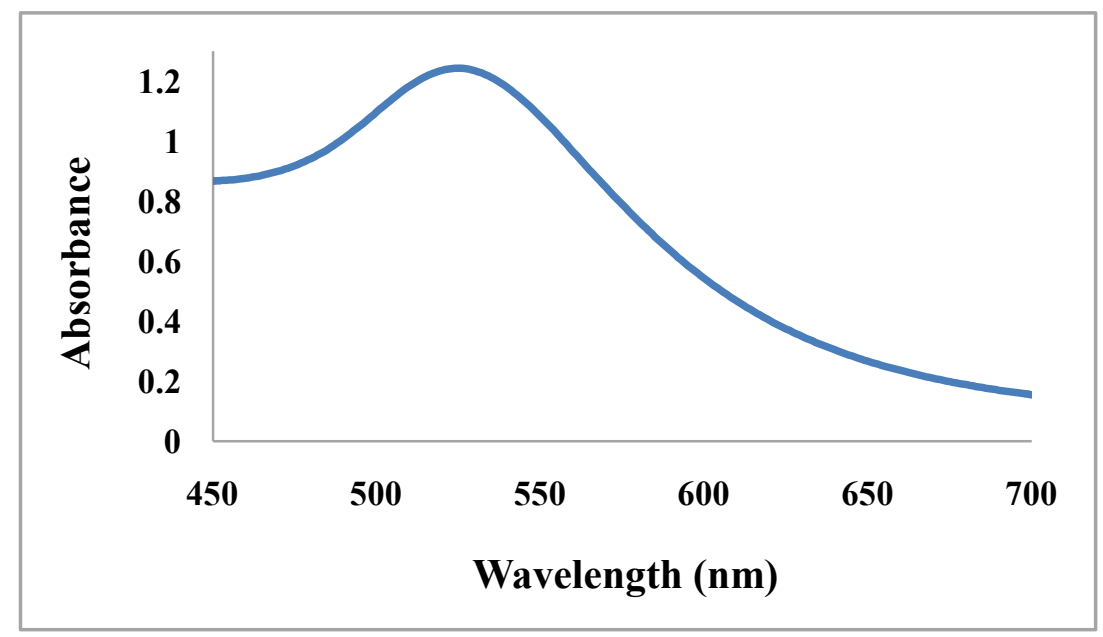

Figure 21: Absorbance spectrum of sodium citrate stabilised GNPs.

The concentration of the particles was calculated as described by Lui et al. (2007) ${ }^{\mathbf{4 0}}$ to be $5.5 \mathrm{nM}$ based on the premise of spherical shaped particles having a uniform face-centered cubic (fcc) structure (refer to section 7.2.1., page 114, for calculation).

The coupling between GNPs and peptides was not successful. On addition of the peptide to the GNP solution, the originally red GNP solution turned a very light blue with the formation of a black precipitate, which is the result of extreme and irreversible aggregation. ${ }^{29}$ An explanation was provided by Tullman et al. (2007): ${ }^{\mathbf{8 6}}$ At $\mathrm{pHs}$ above the $\mathrm{pI}$, the peptides remain bound to the particles. It is likely that the $\mathrm{pH}$ of the solution was below the required $\mathrm{pI}$, leading to insufficient binding of the peptide to GNPs, resulting in their aggregation.

Before this hypothesis could be tested, it was observed that the previously prepared citrate stabilised GNPs began to precipitate. The stabilisation afforded by the citrate ions is insufficient to ensure long term suspension, leading to aggregation. An alternate method of GNP preparation was thus attempted, namely the $\mathrm{NaBH}_{4}$ reduction method. 


\subsubsection{Attachment of peptides to GNPs using the $\mathrm{NaBH}_{4}$ method}

The GNPs were successfully prepared using $\mathrm{NaBH}_{4}$ reduction. The GNP solutions prepared were:

- Au-CKCoum,

- Au-CMECoum,

- Au-CGAFKGGCoum,

- Au-CKAFKRKCoum,

- $\mathrm{Au}-\mathrm{C}(\mathrm{KAFKRK})_{3}$

- $\mathrm{Au}-\mathrm{C}(\mathrm{KAFKRK})_{3} \mathrm{Coum}$,

- $\mathrm{Au}-10 \%$ CKAFKRKCoum:40\% CKAFKRK:50\% C(KAFKRK) 3 .

A variety of peptide sequences were attached to the GNPs and many different outcomes were observed as each peptide affected the morphology of the GNPs in a different manner (Table 7). As discussed in section 2.5. (page 22) many techniques are currently available for the characterization of GNPs. Characterization was performed by means of infrared (IR) spectroscopy, NMR, UV-vis and TEM on all the GNP solutions.

IR and NMR spectroscopy were merely used as a tool to confirm the attachment of ligands and peptides to GNPs. UV-vis was performed to establish the level of dispersity of the GNP solutions. TEM was used to ascertain the size and shape of the GNPs and the disparity of the solution.

\subsubsection{Interpretation of the colour of GNP solutions}

The solutions containing gold salt and peptide were yellow and on addition of $\mathrm{NaBH}_{4}$ rapidly turned dark blue or red, depending on the functionality attached. As described in section 2.2.1. (page 11), a perfectly manufactured colloidal gold solution is wine red in colour, indicating good monodispersity, whereas a poorly manufactured gold solution is blue indicating a polydispersed ${ }^{\mathrm{h}}$ solution. The colour of a GNP solution is thus an indication of aggregation and stability.

GNP solutions capped with CMECoum and CKCoum were blue, which signifies aggregated and polydispersed systems that were not colloidally stable and would inevitably settle over time. The purple colour of the Au-CGAFKGGCoum indicated a polydispersed GNP solution that was aggregated and lacked colloidal stability. The colour of Au-C(KAFRK) $)_{3}$ Coum, Au-C(KAFKRK) 3

\footnotetext{
${ }^{\mathrm{h}}$ A solution is regarded as polydispersed when the particles have a broad range of size and shape.
} 
and $\mathrm{Au}-10 \% \mathrm{CKAFKRKCoum:40 \%} \mathrm{CKAFKRK:50 \%} \mathrm{C}(\mathrm{KAFKRK})_{3}$ were wine red, indicating the presence of well formed, monodispersed and colloidally stable GNP solutions while the colour of Au-CKAFKRKCoum was purple, indicating less monodispersed particles.

\subsubsection{Interpretation of the infrared spectra of the ligand capped GNPs and the free ligands}

IR spectroscopy is a useful tool to confirm the attachment of ligands to GNPs. IR allows for the verification of the structure of the ligand shell attached to the GNPs. This can be done by a simple comparison of the free ligand IR spectrum with that of the GNP capped with the ligand. The method of attachment of a ligand to GNPs can also be determined by examination of their spectra. Ligands attached to GNPs through a thiol group lack the - SH functionality present in the spectra of the neat capping ligands.

The FTIR spectra for the GNP samples were compared with those of the corresponding neat capping peptides to confirm their attachment to GNPs (Appendix 2, page 145-150).

In the case of CMECoum and Au-CMECoum [Figure 22 and Figure 23 (page 47) respectively] both the free peptide and peptide capped GNPs showed bands at $\sim 1700 \mathrm{~cm}^{-1}$ from the $\mathrm{C}=\mathrm{O}$ stretching band of the amide I band region. The peaks appearing at $1547 \mathrm{~cm}^{-1}$ can be assigned to the N-H bending and $\mathrm{C}-\mathrm{N}$ stretching in the amide II band region. The bands due to $\mathrm{C}-\mathrm{NH}_{2}$ stretching appeared near $1400 \mathrm{~cm}^{-1}$ as well as various weaker bands relate to $\mathrm{C}-\mathrm{N}, \mathrm{C}-\mathrm{O}$ vibration and amino acid residues (under $1350 \mathrm{~cm}^{-1}$ ). It was observed that the $-\mathrm{SH}$ stretching frequency $\left(2550-2600 \mathrm{~cm}^{-}\right.$

${ }^{1}$ ) of the bound surfactants disappeared in the MPC. This suggests that the bonding of the CMECoum to the Au surface had taken place through the $-\mathrm{SH}$ end.

The same trend was observed for all the functionalised GNPs (the ligands attached to GNPs consist of the same functional groups and thus yield similar IR spectra) as evident in the spectra (Appendix 2, page 145-150). The presence of similar peaks in the IR spectra of the neat ligands as in the ligand capped GNPs confirm the covalent binding of the ligands to the GNPs. 


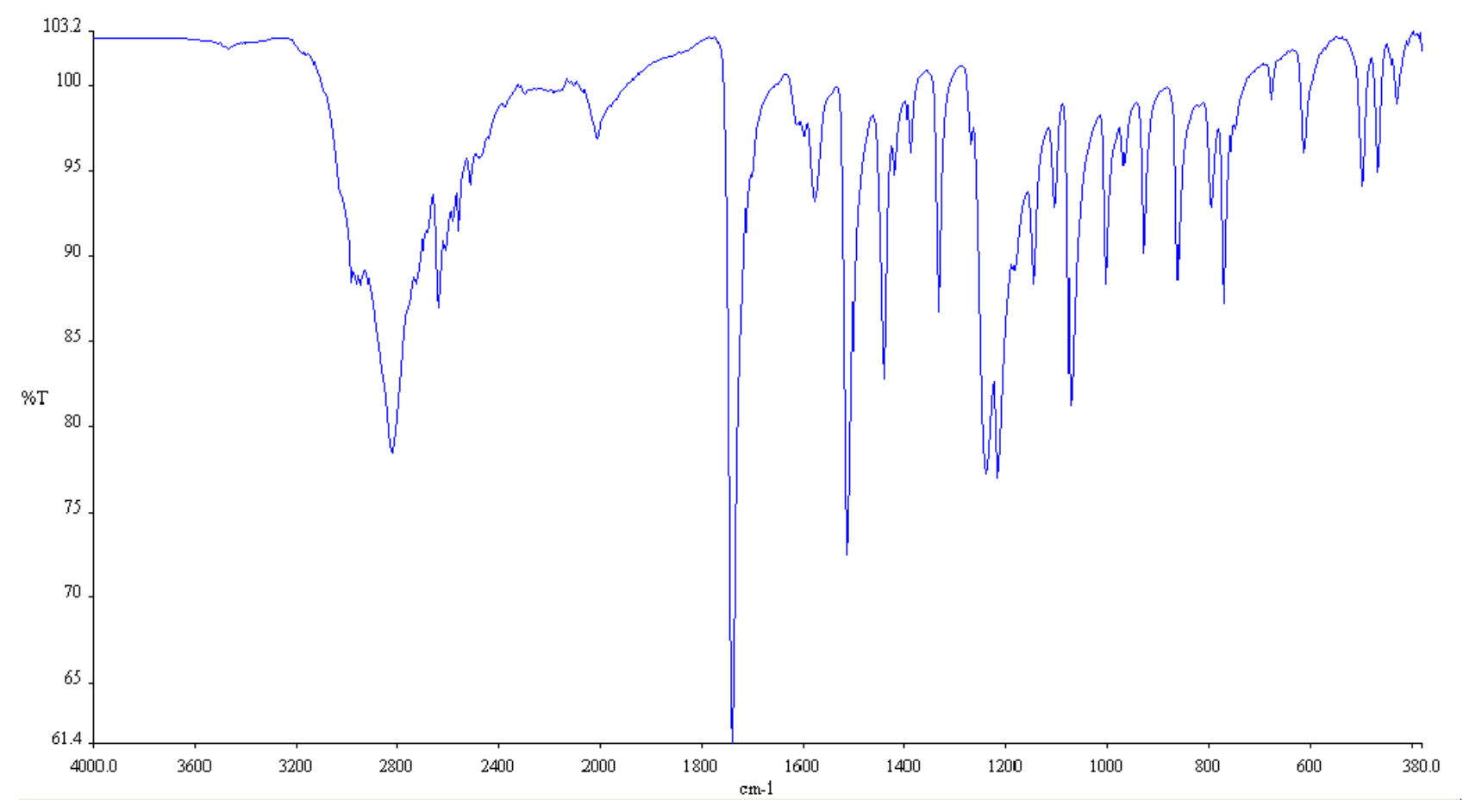

Figure 22: ATR-FTIR spectrum of CMECoum.

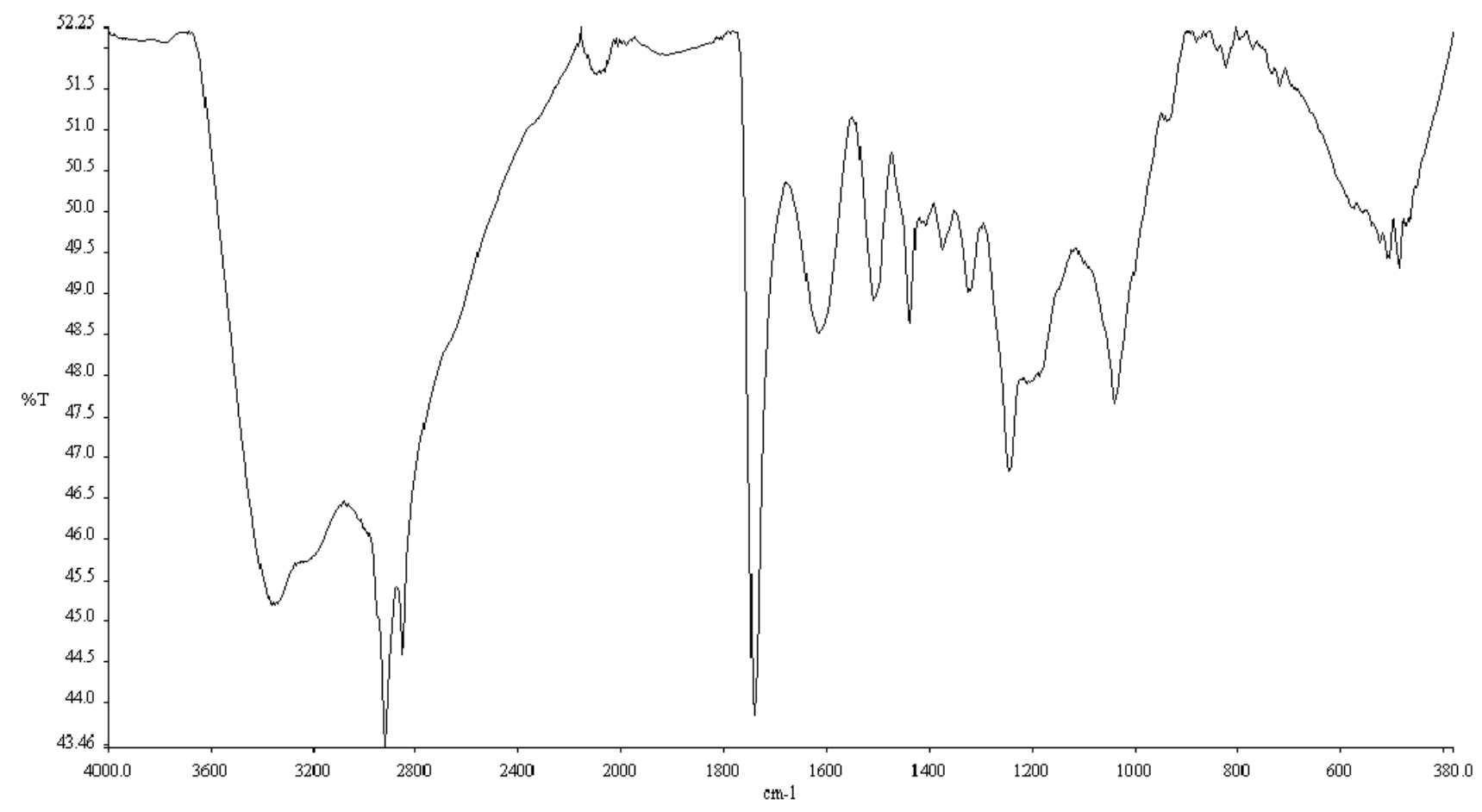

Figure 23: ATR-FTIR spectrum of Au-CMECoum. 


\subsubsection{Interpretation of the NMR spectra of the ligand capped GNPs and the free ligands}

A prerequisite for assigning the proton NMR spectrum of a polypeptide is to be familiar with the chemical structure of the side chains of the amino acid residues present in the sequence, as well as with the chemical shifts expected for every chemically different proton in the molecule. ${ }^{\mathbf{8 7}}$ The side chains of amino acids present in the peptide sequence are shown in Table 5 (glycine does not have a side chain and thus is not included in the table). The usual chemical shifts observed for each different type of proton are summarized in Table 6.

Table 5: Side chains of the amino acid present in the peptide sequence CKAFKRK. For each residue the full amino acid name is shown. ${ }^{87}$

\begin{tabular}{|ccc|}
\hline Amino Acid & One letter code & Side Chain \\
\hline Alanine & $\mathrm{A}$ & $\mathrm{CH}_{3}$ \\
\hline Arginine & $\mathrm{R}$ & $\mathrm{CH}_{2}-\mathrm{CH}_{2}-\mathrm{CH}_{2}-\mathrm{NH}-\mathrm{C}-\left(\mathrm{NH}_{2}\right)_{2}$ \\
\hline Cysteine & $\mathrm{C}$ & $\mathrm{CH}_{2}-\mathrm{SH}$ \\
\hline Lysine & $\mathrm{K}$ & $\mathrm{CH}_{2}-\mathrm{CH}_{2}-\mathrm{CH}_{2}-\mathrm{CH}_{2}-\mathrm{NH}_{2}$ \\
\hline Phenylalanine & $\mathrm{F}$ & $\mathrm{Ph}_{-}-\mathrm{CH}_{2}-\mathrm{CH}\left(\mathrm{NH}_{2}\right)-\mathrm{COOH}$ \\
\hline
\end{tabular}


Table 6: Summary of usual chemical shift ranges for protons in the side chains of the amino acid residues CKAFKRK. ${ }^{87}$

\begin{tabular}{|c|c|}
\hline$\delta(\mathbf{p p m})$ & Type of proton \\
\hline 0.9-1.4 & $\mathrm{CH}_{3}$ of alanine and cysteine methyl ester \\
\hline 1.1-1.9 & $\mathrm{CH}$ proton of Coumarin \\
\hline $1.2-2.3$ & $\begin{array}{c}\mathrm{CH}_{2} \text { and } \mathrm{CH} \text { with no neighbouring } \\
\text { electronegative or aromatic groups }\left(\mathrm{BCH}_{2} \text { of }\right. \\
\text { lysine and arginine; } \gamma \mathrm{CH}_{2} \text { of lysine and } \\
\left.\text { arginine; } \delta \mathrm{CH}_{2} \text { of lysine }\right)\end{array}$ \\
\hline 2.3-3.7 & $\begin{array}{l}\mathrm{CH}_{2} \text { with neighbouring aromatic or carbonyl } \\
\text { groups or nitrogen or sulfur atoms }\left(\beta \mathrm{CH}_{2} \text { of }\right. \\
\text { cysteine and phenylalanine; } \delta \mathrm{CH}_{2} \text { of } \\
\left.\text { arginine; } \varepsilon \mathrm{CH}_{2} \text { of lysine }\right)\end{array}$ \\
\hline $3.9-4.8$ & $\alpha \mathrm{CH}$ and $\alpha \mathrm{CH}_{2}$ \\
\hline $6.6-7.6$ & $\mathrm{NH}$ in side chains of lysine and arginine \\
\hline $6.8-7.7$ & $\begin{array}{l}\text { Aromatic } \mathrm{CH} \text { protons of phenylalanine and } \\
\text { Coumarin }\end{array}$ \\
\hline 7.9-8.8 & Backbone NH \\
\hline
\end{tabular}

Proton NMR measurements were performed on the neat peptides, and on the peptide functionalised GNPs in $\mathrm{D}_{2} \mathrm{O}$ and in $\mathrm{CD}_{3} \mathrm{OD}$ for CMECoum and Au-CMECoum (refer to Appendix 2, page 151155 , for spectra).

\section{CMECoum}

The peaks in the region of 0.9-1.4 ppm correspond to the $\mathrm{CH}_{3}$ of methyl ester. The peaks in the region of 1.1-1.9 ppm correspond to the $\mathrm{CH}$ proton of the Coumarin moiety. The peaks in the region of $2.3 \mathrm{ppm}$ correspond to the $\mathrm{BCH}_{2}$ of cysteine. The peaks in the region of 3.5-4.3 ppm correspond to the $\alpha \mathrm{CH}$ and $\alpha \mathrm{CH}_{2}$. The peaks at 3.3 and $4.7 \mathrm{ppm}$ are the solvent peaks of $\mathrm{CD}_{3} \mathrm{OD}$. The peaks in the region of 7.0-7.8 ppm correspond to the aromatic $\mathrm{CH}$ protons of Coumarin. The peaks in the region of 8.7 ppm correspond to the backbone NH protons. ${ }^{87}$ Refer to page 151 for the NMR specrum. 


\section{Au-CMECoum}

A variety of deuterated solvents $\left(\mathrm{CDCl}_{3}, \mathrm{CD}_{3} \mathrm{OD}, \mathrm{D}_{2} \mathrm{O}\right)$ were used in an attempt to solubilise the CMECoum capped GNPs. In each solvent the capped GNPs showed little sign of dissolution and settled to the bottom of the NMR tube. Due to the poor solubility of the ligand capped GNPs in appropriate solvents, thorough NMR studies were not feasible. Refer to page 151 for the NMR spectrum.

\section{CKCoum}

The peaks in the region of 1.1-1.9 ppm correspond to the $\mathrm{\beta CH}_{2}$ protons of lysine, the $\gamma \mathrm{CH}_{2}$ protons of lysine and the $\mathrm{CH}$ proton of the Coumarin moiety. The peaks in the region of 2.7-2.9 ppm correspond to the $\beta_{\mathrm{CH}_{2}}$ of cysteine and the $\varepsilon \mathrm{CH}_{2}$ of lysine. The peaks in the region of 3.9-4.6 ppm correspond to the $\alpha \mathrm{CH}$ and $\alpha \mathrm{CH}_{2}$. The peak at $4.7 \mathrm{ppm}$ is the solvent peak $\mathrm{D}_{2} \mathrm{O}$. The peaks in the region of 7.0-7.8 ppm correspond to the aromatic $\mathrm{CH}$ protons of Coumarin. The peaks in the region

of 8.2-8.7 ppm correspond to the NH protons involved in hydrogen bonding. ${ }^{87}$ The NH resonances of peptides dissolved in $\mathrm{D}_{2} \mathrm{O}$ are expected to exchange in the presence of the solvent. This is not always the case, however, as the high affinity of peptides to water prevents the removal of all traces of water from the sample, thereby resulting in the presence of $\mathrm{NH}$ resonances in the NMR spectrum. Refer to page 152 for the NMR spectrum.

\section{Au-CKCoum}

As with Au-CMECoum, a variety of deuterated solvents were used in an attempt to solubilise the CKCoum capped GNPs. In each solvent the capped GNPs little no sign of dissolution and completely settled to the bottom of the NMR tube. Due to the poor solubility of the ligand capped GNPs in appropriate solvents, thorough NMR studies were not feasible. Refer to page 152 for the NMR spectrum.

\section{CGAFKGGCoum, CKAFKRKCoum, Au-C(KAFKRK) $)_{3}$ and C(KAFKRK) $)_{3}$ Coum}

It was anticipated that the peptides CKAFKRKCoum, C(KAFKRK $)_{3}$ and C(KAFKRK $)_{3}$ Coum would have similar proton NMR spectra, as the peptide sequences consist of the same amino acids. The peptide CGAFKGGCoum differs slightly from the other two peptides by the presence of a glycine and lack of an arginine residue. These differences should have limited effect on the resultant proton NMR spectrum as glycine does not have a side chain and the lysine residue would 
resonate in the same region as arginine. As a result, all three peptide sequences are expected to have very similar proton NMR spectra.

The following elucidation applies for all three peptides. The peaks at $\sim 1.1 \mathrm{ppm}$ correspond to the $\mathrm{CH}_{3}$ protons of alanine. The peaks in the region of 1.1-1.9 ppm correspond to the $\mathrm{CH}$ proton of the Coumarin moiety. The peaks at 1.2-1.9 ppm can be assigned to the $\mathrm{\beta CH}_{2}$ protons of lysine and arginine and the $\gamma \mathrm{CH}_{2}$ protons of lysine and arginine and the $\delta \mathrm{CH}_{2}$ of lysine. The peaks in the region of 2.7-3.4 ppm correspond to the $\beta_{\mathrm{CH}_{2}}$ of cysteine and phenylalanine and the $\delta \mathrm{CH}_{2}$ of arginine and the $\varepsilon \mathrm{CH}_{2}$ of lysine. The peaks in the region of 3.9-4.6 ppm correspond to the $\alpha \mathrm{CH}$ and $\alpha \mathrm{CH}_{2}$. The peak at $4.7 \mathrm{ppm}$ is the solvent peak $\mathrm{D}_{2} \mathrm{O}$. The peaks in the region of 7.0-7.5 ppm correspond to the aromatic $\mathrm{CH}$ protons of phenylalanine and Coumarin. The peaks in the region of 7.9-8.3 ppm correspond to the backbone $\mathrm{NH}$ protons. ${ }^{87}$ As mentioned previously, the $\mathrm{NH}$ resonances of peptides dissolved in $\mathrm{D}_{2} \mathrm{O}$ are expected to exchange in the presence of the solvent. This is not always the case, however, as the high affinity of peptides to water prevents the removal of all traces of water from the sample, thereby resulting in the presence of $\mathrm{NH}$ resonances in the NMR spectrum. Refer to Spectrum 1 (page 52) for ${ }^{1} \mathrm{H}$ NMR spectrum of CKAFKRKCoum in $\mathrm{D}_{2} \mathrm{O}$ and Appendix 2, page 153-155 for the remainder NMR spectra.

\section{Au-CGAFKGgCoum, Au-CKAFKRKCoum, Au-C(KAFKRK) $)_{3}$, Au-C(KAFKRK) $)_{3}$ Coum and Au-10\% CKAFKRKCOUm:40\% CKAFKRK:50\% C(KAFKRK)}

As with Au-CMECoum, a variety of deuterated solvents were used in attempts to solubilise the CGAFKGGCoum capped GNPs. In each solvent the capped GNPs showed no sign of dissolution and settled to the bottom of the NMR tube. Due to the poor solubility of the ligand capped GNPs in appropriate solvents, thorough NMR studies were not feasible.

A comparison of the ${ }^{1} \mathrm{H}$ NMR spectra recorded for the CKAFKRKCoum, C(KAFKRK $)_{3}$ and $\mathrm{C}(\mathrm{KAFKRK})_{3}$ Coum peptides and the GNP composites Au-CKAFKRKCoum, Au-C(KAFKRK) $\mathrm{Au}-\mathrm{C}(\mathrm{KAFKRK})_{3} \mathrm{Coum}$ and $\mathrm{Au}-10 \%$ CKAFKRKCoum:40\% CKAFKRK:50\% C(KAFKRK) 3 show remarkable similarity. NMR spectra of the peptide-derivatised GNPs are consistent with the peptide sequences being bound to the colloidal gold surface, providing a clear indication of the attachment of peptide to GNP. Refer to Spectrum 2 (page 52) for ${ }^{1} \mathrm{H}$ NMR spectrum of AuCKAFKRKCoum in $\mathrm{D}_{2} \mathrm{O}$ and Appendix 2, page 153-155 for the remainder NMR spectra. 


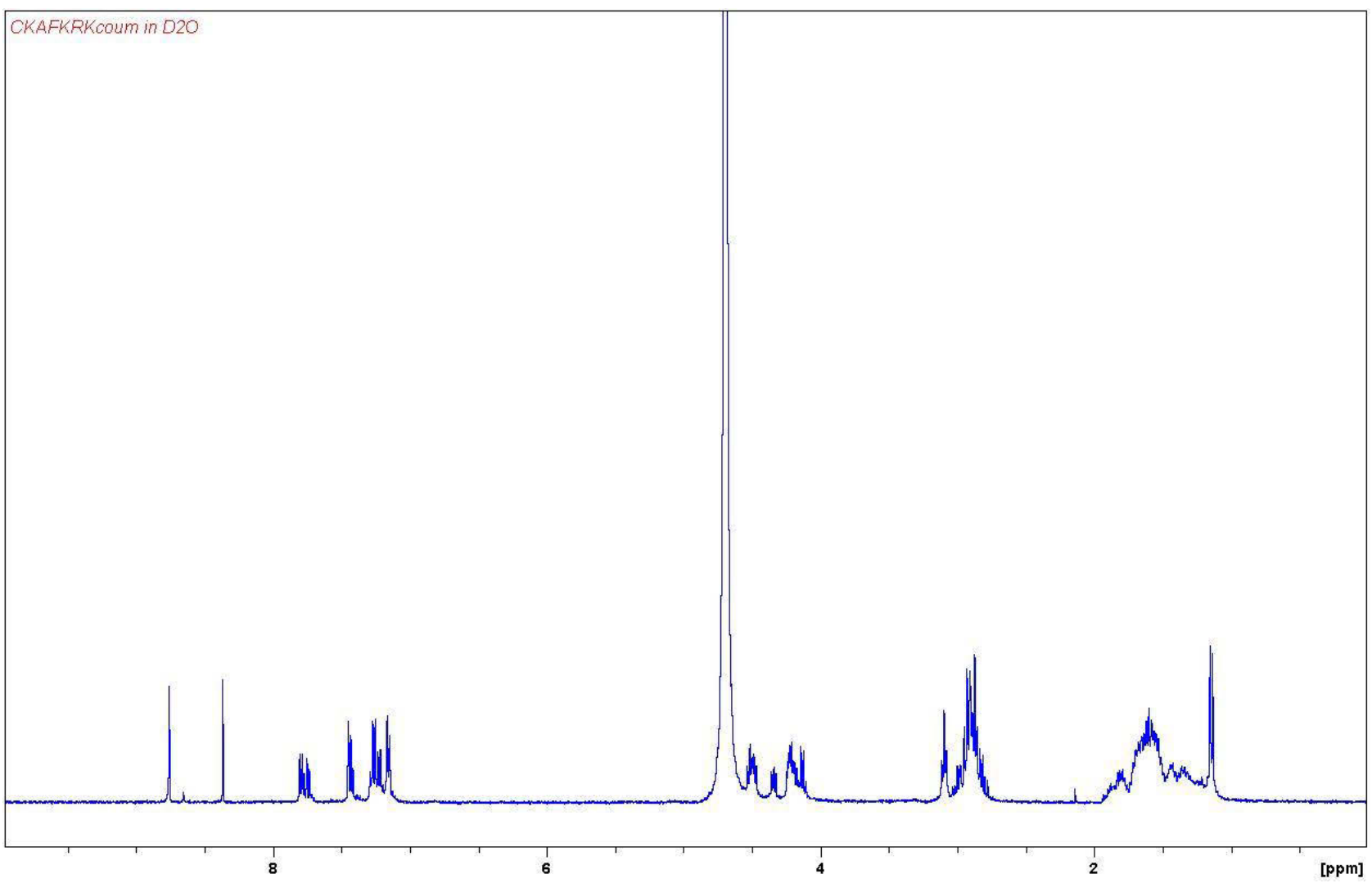

Spectrum 1: ${ }^{1} \mathrm{H}$ NMR spectrum of CKAFKRKCoum in $\mathrm{D}_{2} \mathrm{O}$.

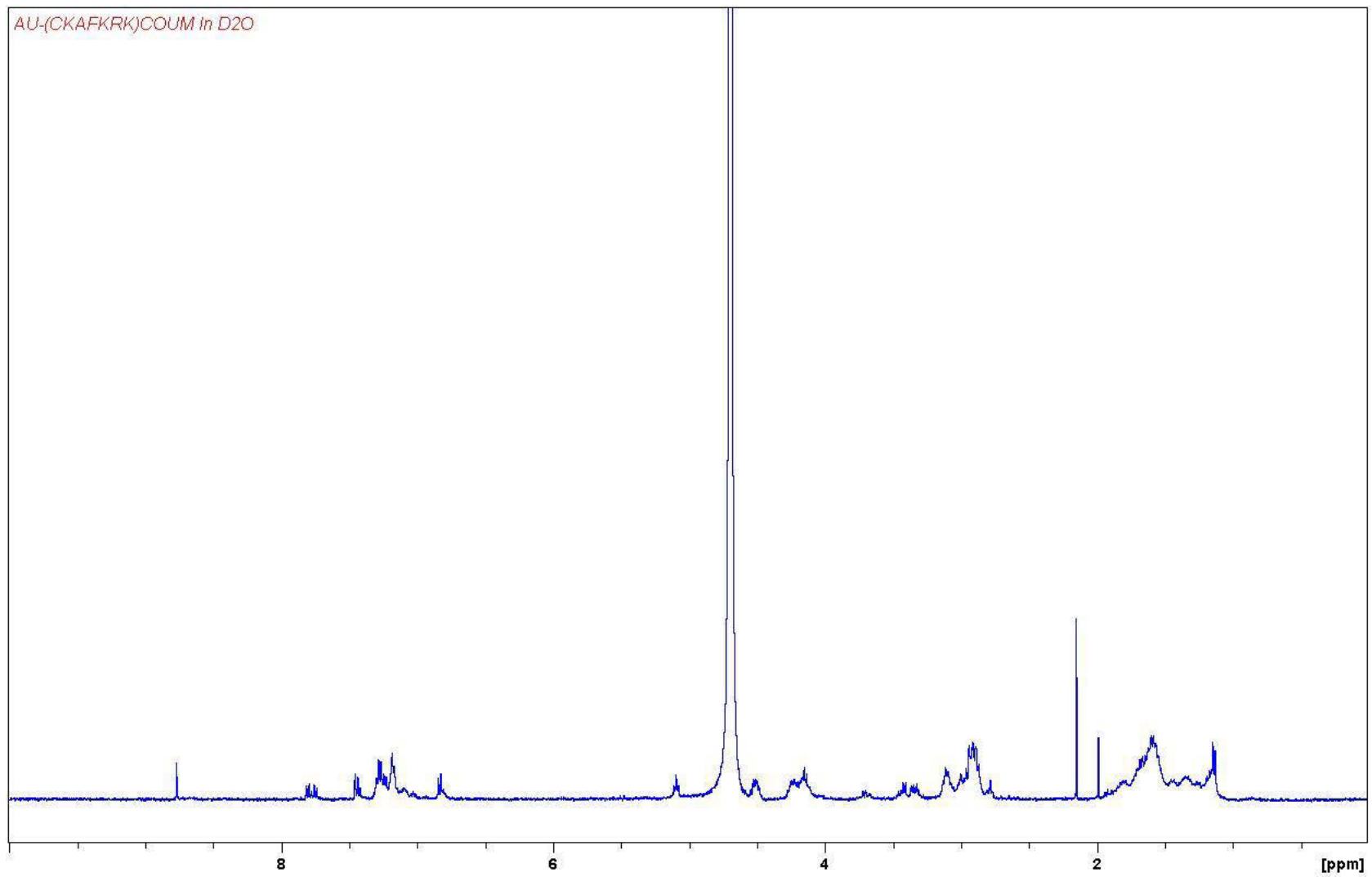

Spectrum 2: ${ }^{1} \mathrm{H}$ NMR spectrum of Au-CKAFKRKCoum in $\mathrm{D}_{2} \mathrm{O}$. 
Peak broadening and splitting is usually noted in the ${ }^{1} \mathrm{H}$ NMR spectra of peptide capped gold composites. ${ }^{88}$ Comparison of the ${ }^{1}$ H NMR spectra of peptide capped GNPs and the respective free peptide indicates that the peaks appear to have collapsed to a single broad peak in the peptide capped GNPs, as opposed to the sharp peaks evident in the spectra of the free peptides. Peak splitting is due to the formation of hydrogen bonding between the surfaces of bound amino acid molecules of neighbouring amino acid capped GNPs. No peak splitting was evident in the ${ }^{1} \mathrm{H}$ NMR spectra, indicating the lack of hydrogen bonding between adjacent amino acids.

\subsubsection{Interpretation of the UV-vis spectra of the ligand capped GNPs}

Au-CMECoum, Au-CKCoum and Au-CGAFKGGCoum samples were studied by UV-vis spectroscopy [Figure 24, Figure 25 and Figure 26 (page 54) respectively]. For the Au-CMECoum sample, the plasmon band was shifted to $\sim 570 \mathrm{~nm}$. For the Au-CKCoum sample, a broad and less prominent plasmon band was observed in the region of 550-800 nm. For the Au-CGAFKGGCoum sample the absorption band was broadened and red shifted to $600 \mathrm{~nm}$.

Perfectly monodispersed GNPs show a plasmon resonance band at $520 \mathrm{~nm}$. The shifted and broadened bands are the result of aggregation/polydispersity present in the samples. "The aggregation of the particles in the samples results in an increased overlap of surface plasmon resonance from neighbouring GNPs that results in a spectral change of the band" (Storhoff, $1998^{76}$ ).

$\mathrm{Au}-\mathrm{C}(\mathrm{KAFKRK})_{3} \mathrm{Coum}, \quad \mathrm{Au}-\mathrm{C}(\mathrm{KAFKRK})_{3}, \quad \mathrm{Au}-\mathrm{CK}$ AFKRKCoum $\quad$ and $\mathrm{Au}-10 \%$ CKAFKRKCoum:40\% CKAFKRK:50\% $\quad$ C(KAFKRK) $)_{3}$ samples were studied by UV-vis spectroscopy [Figure 27, - Figure 29 (page 55) respectively]. As anticipated, the spectra of Au$\mathrm{C}(\mathrm{KAFKRK})_{3} \mathrm{Coum}$ and $\mathrm{Au}-\mathrm{C}(\mathrm{KAFKRK})_{3}$ were identical thus only Au-C(KAFKRK $)_{3} \mathrm{Coum}$ is displayed. All three spectra showed a strong and prominent absorption band at $520 \mathrm{~nm}$, indicating that red-shifting and broadening did not occur. It can be deduced that the particles did not coalesce to any extent. 


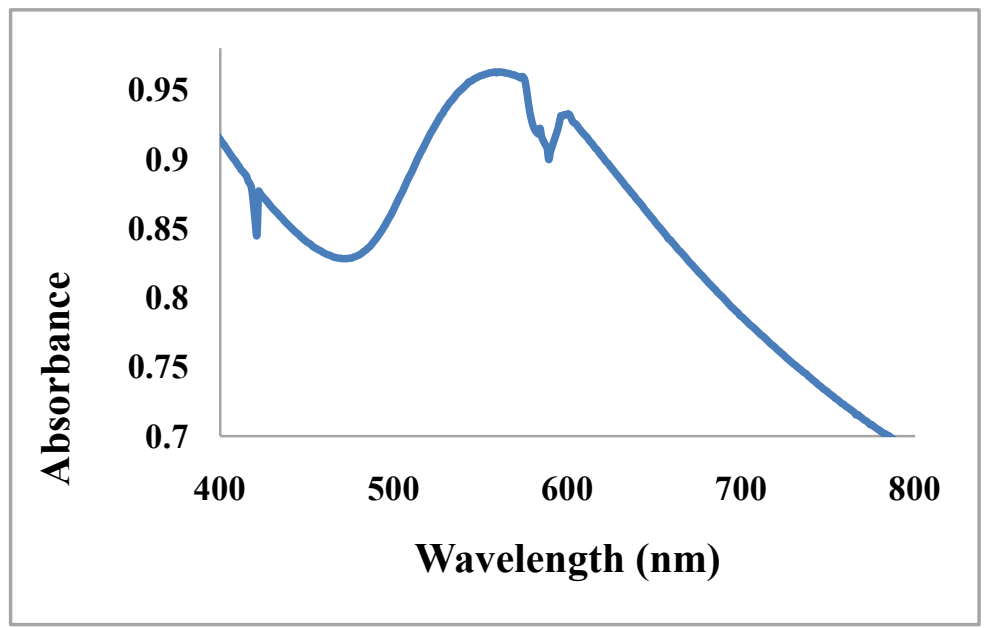

Figure 24: Absorbance spectrum of Au-CME.

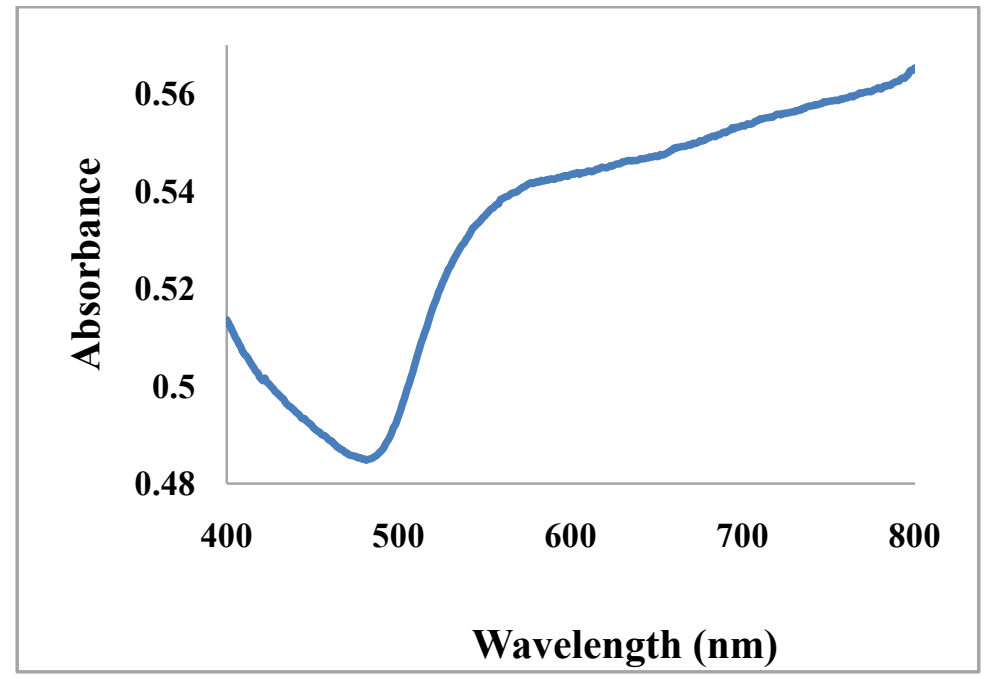

Figure 25: Absorbance spectrum of Au-CKCoum.

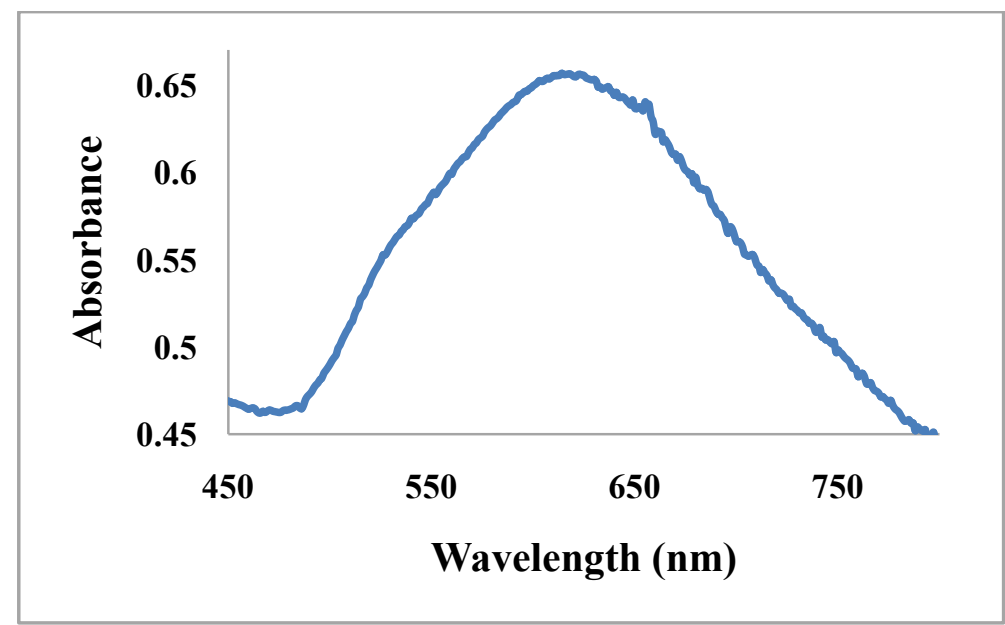

Figure 26: Absorbance spectrum of Au-CGAFKGGCoum. 


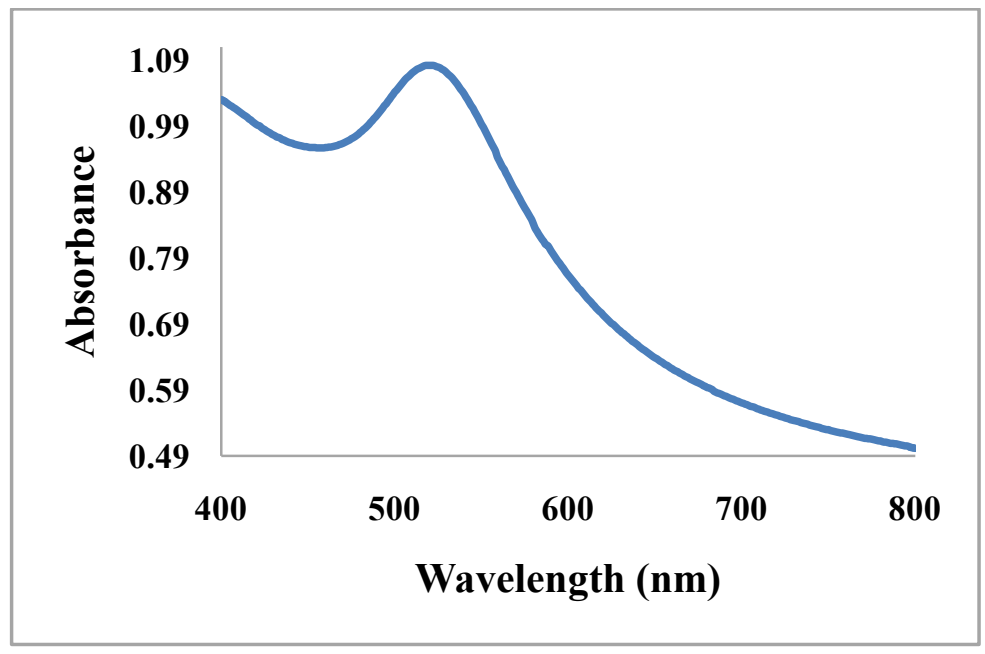

Figure 27: Absorbance spectrum of $\mathrm{Au}-\mathrm{C}(\mathrm{KAFKRK})_{3} \mathrm{Coum}$.

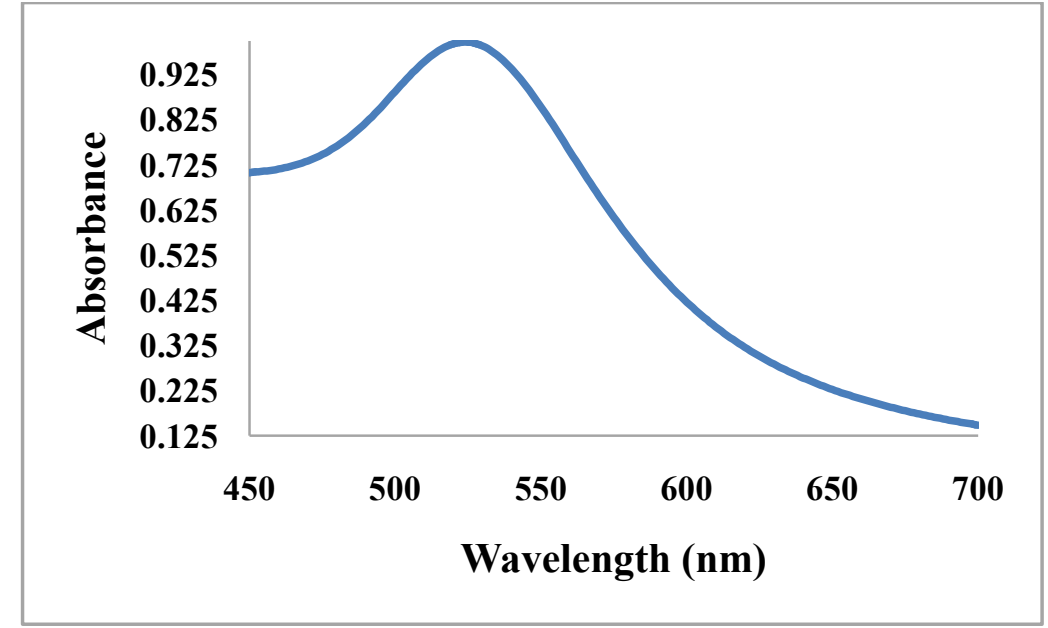

Figure 28: Absorbance spectrum of Au-CKAFKRKCoum.

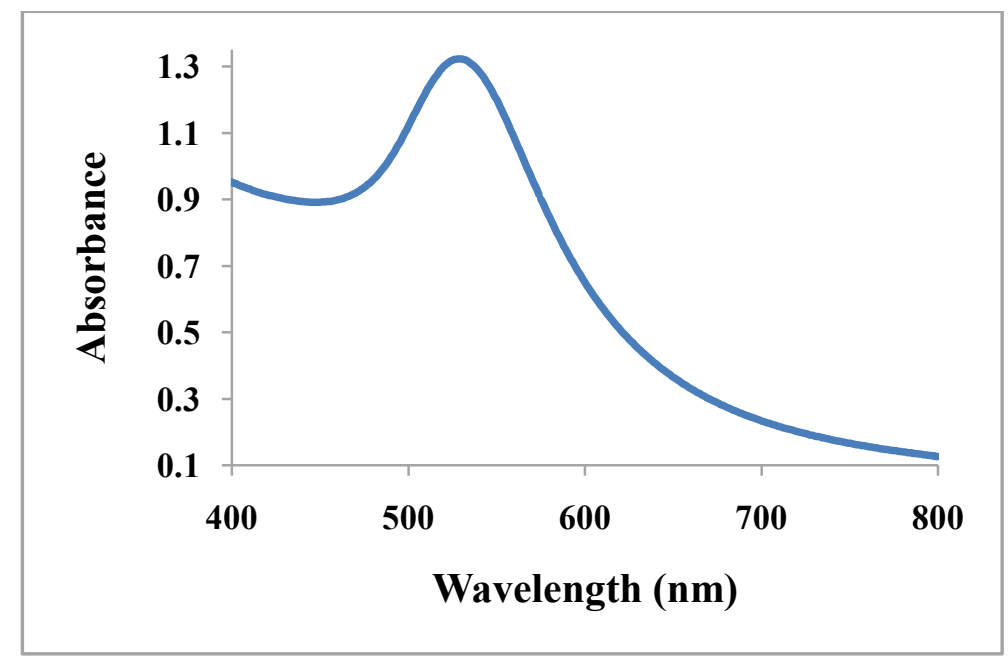

Figure 29: Absorbance spectrum of Au-10\% CKAFKRKCoum:40\% CKAFKRK:50\% $\mathrm{C}(\mathrm{KAFKRK})_{3}$. 
TEM was applied to characterise the morphology and size of the GNPs and is discussed in detail.

\subsubsection{Interpretation of the TEM images of the ligand capped GNPs}

TEM images indicated that the extent of aggregation in both CME and CKCoum was severe (Figure $30 \mathrm{~A}$ and Figure $30 \mathrm{~B}$ respectively). The coalescence complicated the accurate determination of shape and size of the NPs. The few free spherical particles were measured and found to be approximately $9.6 \mathrm{~nm}$ for the Au-CKCoum and $13.2 \mathrm{~nm}$ for the Au-CMECoum.
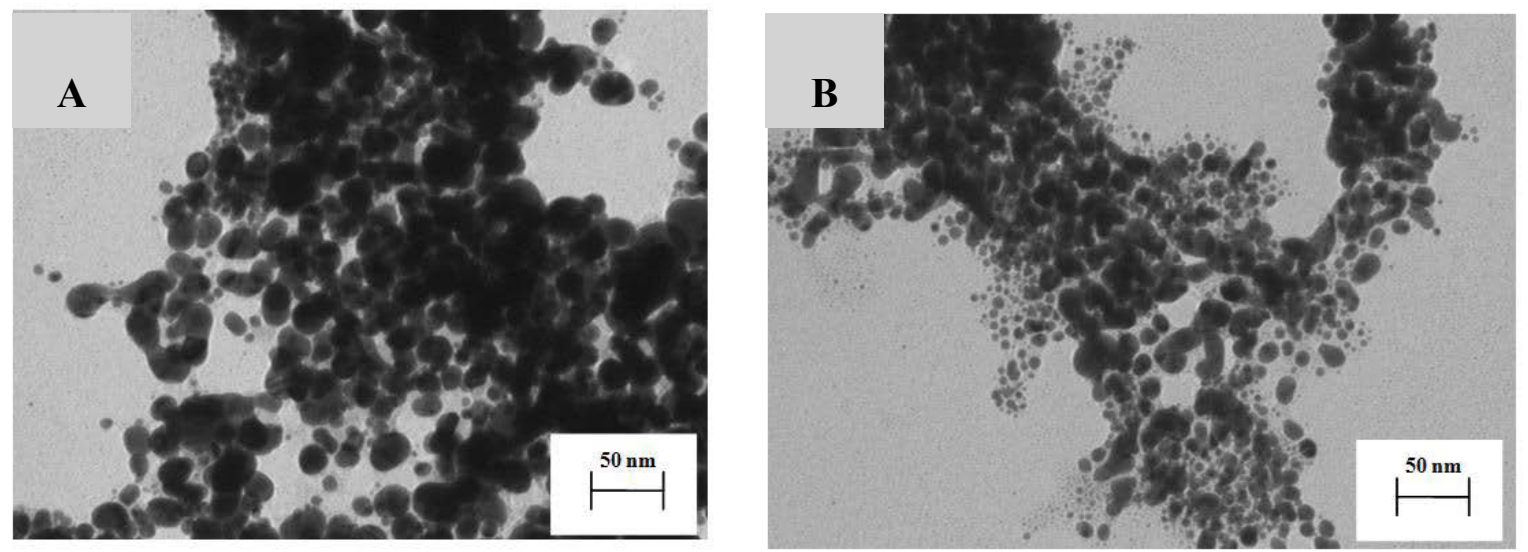

Figure 30: TEM images of Au-CME (A) and Au-CKCoum (B) NP dispersions in distilled water.

The Au-CGAFKGGCoum sample was studied with TEM and again the extent of aggregation was found to be severe. A few free particles were spherical in shape and about $14.7 \mathrm{~nm}$ in size (Figure $31)$.

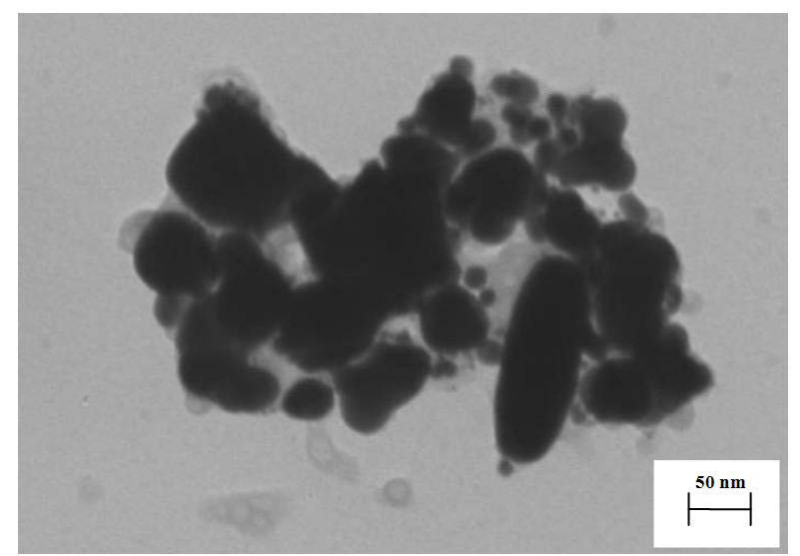

Figure 31: TEM image of Au-CGAFKGGCoum.

The Au-CKAFKRKCoum, Au-C(KAFKRK) $)_{3}$ Coum and Au-10\% CKAFKRKCoum:40\% CKAFKRK:50\% C(KAFKRK) $)_{3}$ samples were observed under TEM. (As anticipated, the TEM 
images of $\mathrm{Au}-\mathrm{C}(\mathrm{KAFKRK})_{3} \mathrm{Coum}$ and $\mathrm{Au}-\mathrm{C}(\mathrm{KAFKRK})_{3}$ were identical thus only Au$\mathrm{C}(\mathrm{KAFKRK})_{3}$ Coum is displayed). As shown in Figure $32 \mathrm{~A}-\mathrm{B}$, the NPs are generally spherical and well separated from each other demonstrating good monodispersity. The average core diameters were calculated for each sample by measuring the core diameters of 200 particles from multiple micrographs to ensure statistical relevance. The core diameters of Au-CKAFKRKCoum, AuC(KAFKRK) ${ }_{3}$ Coum and Au-10\% CKAFKRKCoum:40\% CKAFKRK:50\% C(KAFKRK) $)_{3}$ samples were $11.9 \mathrm{~nm}, 12.6 \mathrm{~nm}$ and $15.2 \mathrm{~nm}$ respectively. It is noted that the Au-CKAFKRKCoum is less monodispersed than $\mathrm{Au}-\mathrm{C}(\mathrm{KAFKRK})_{3} \mathrm{Coum}$ with the presence of larger as well as smaller particles.
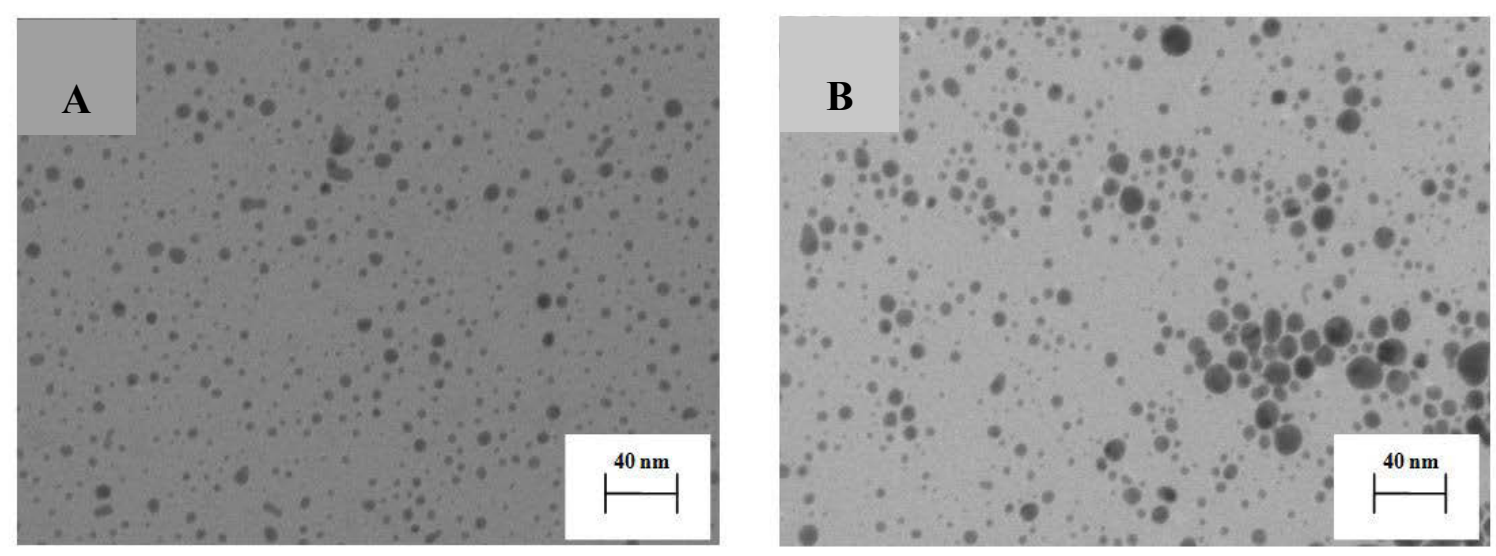

Figure 32: $\mathrm{TEM}$ images of $\mathrm{Au}-\mathrm{C}(\mathrm{KAFKRK}){ }_{3} \mathrm{Coum}(\mathrm{A})$ and $\mathrm{Au}-\mathrm{CKAFKRKCoum}(\mathrm{B})$ in distilled water.

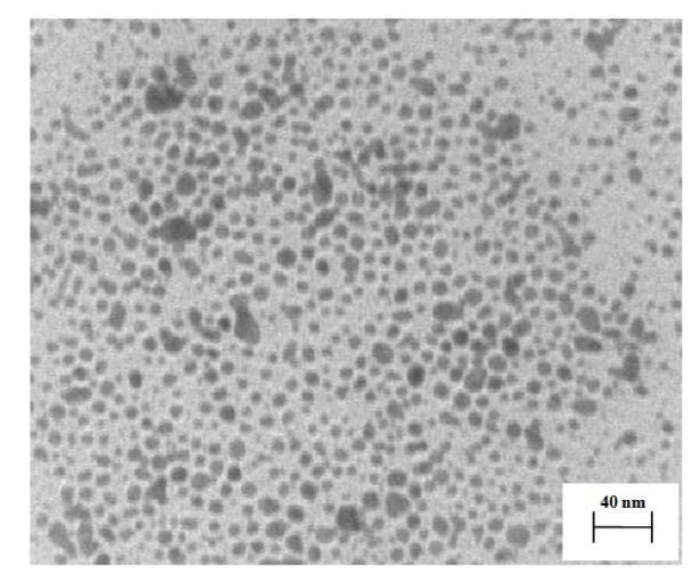

Figure 33: TEM image of Au-10\% CKAFKRKCoum:40\% CKAFKRK:50\% C(KAFKRK) 3 capped in distilled water. 


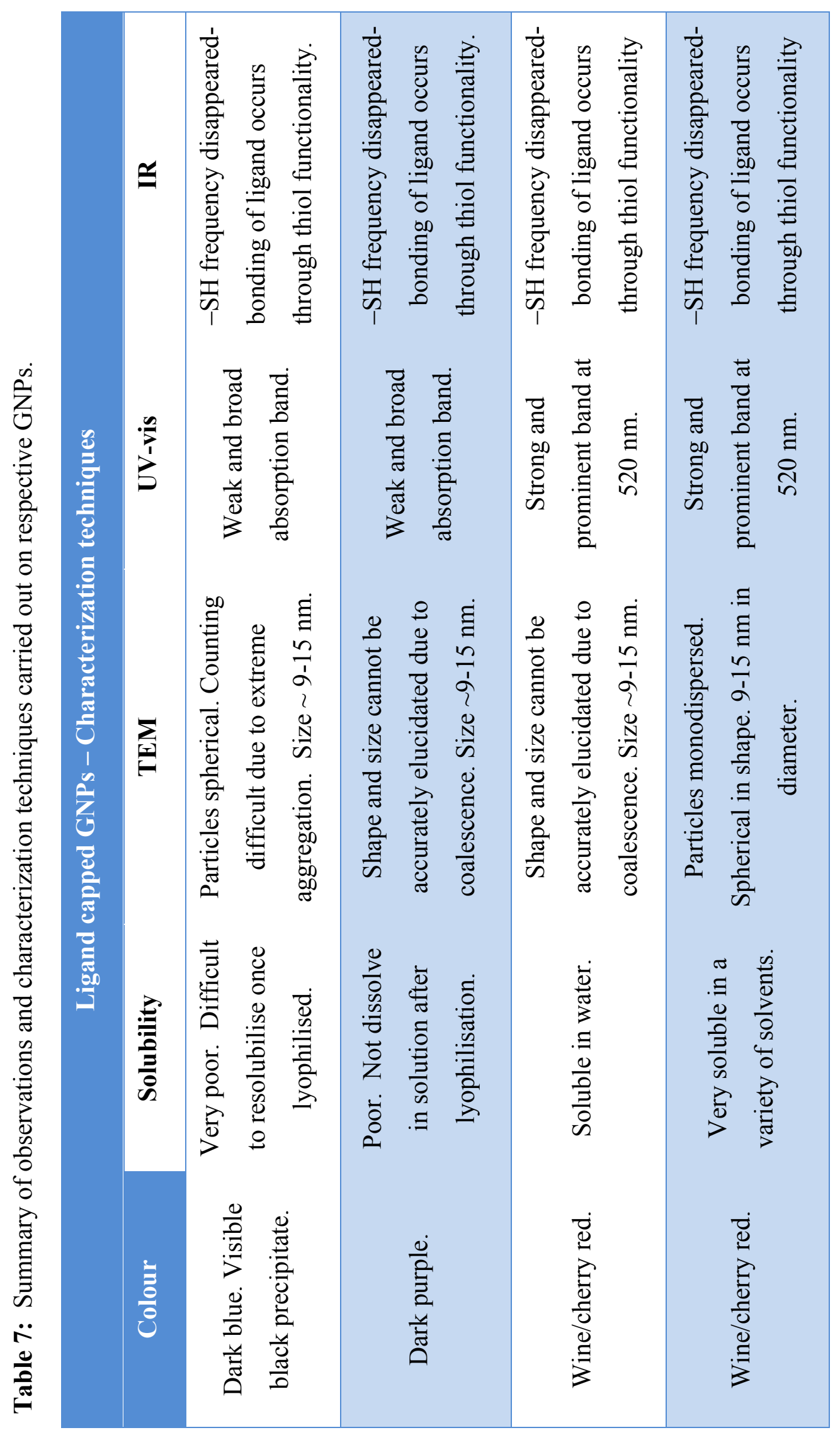




\subsubsection{Size of GNPs}

As mentioned in section 4.1.1. (page 34) the size of the GNPs should be strictly controlled. GNP sizes can be altered by adjusting the $\mathrm{Au}$ :thiol and the $\mathrm{Au}: \mathrm{NaBH}_{4}$ ratios. The amount of reducing agent required is well established and most mention the use of ten times the molar equivalent of $\mathrm{HAuCl}_{4}$. The ratio of Au:thiol is not standardised as it is highly dependent on the thiol of choice. Various Au:CKAFKRKCoum ratios were attempted to determine the resultant GNP sizes (Table 8).

Table 8: The effect of the Au:thiol ratio on the average size of gold NPs.

\begin{tabular}{|cc|}
\hline Au:Thiol ratio (mol:mol) & Size (nm) \\
\hline $\mathbf{6 : 1}$ & 20 \\
\hline $\mathbf{4 : 1}$ & 17 \\
\hline $\mathbf{2 : 1}$ & 15 \\
\hline $\mathbf{1 : 1}$ & 12 \\
\hline $\mathbf{0 . 5 : 1}$ & 12 \\
\hline
\end{tabular}

A calibration curve was constructed in order to illustrate the relationship between the Au:thiol ratio ( $\mathrm{x}$ azis) and the average size of the GNPs (y axis). 


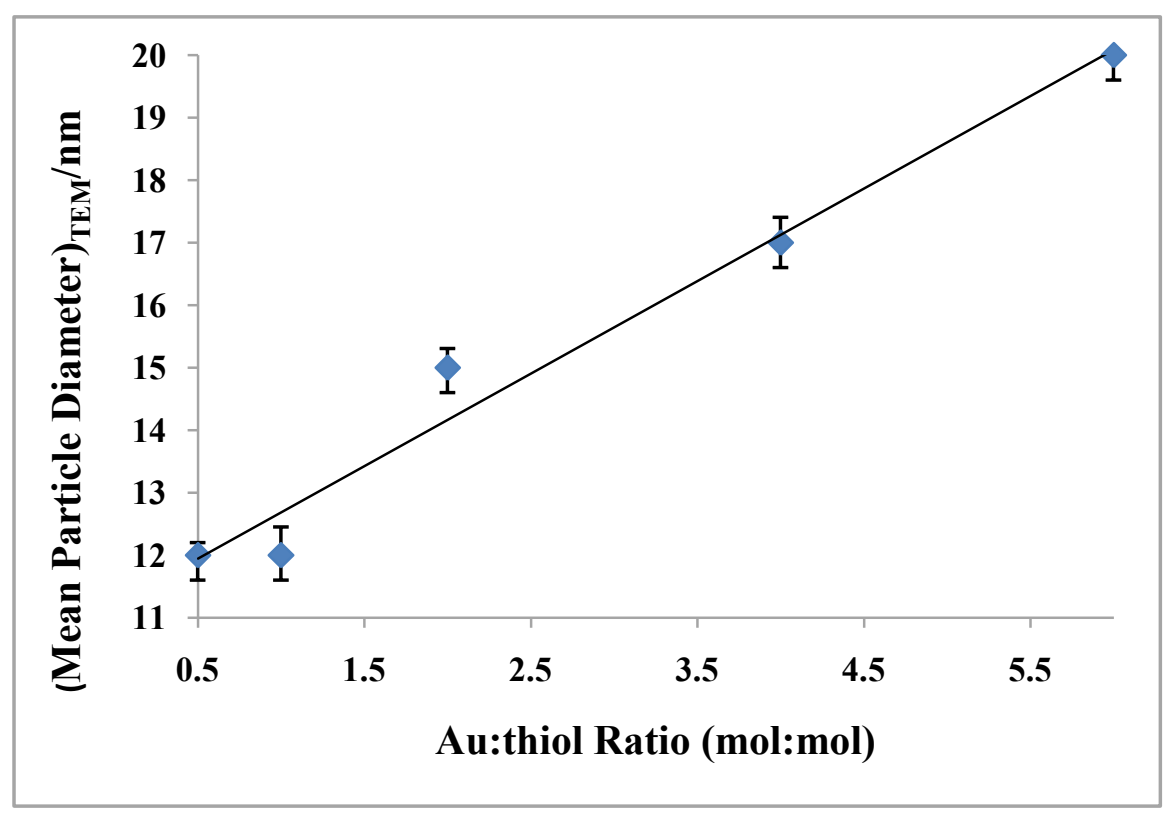

Figure 34: NP size analysis of GNPs prepared with varying Au:thiol molar ratios in the synthesis mixture, highlighting an increase in mean NP core diameter with increasing Au:thiol molar ratio. Each data point is representative of an average of three experimental assays.

As mentioned in section 4.1.1. (page 34) the Au:thiol ratio is incrementally proportional to the GNP particle size. As expected, the increase in Au:thiol ratio resulted in larger particle sizes (direct relationship shown in Figure 34). It was also noted that at ratio values lower than $2: 1$, the particle size stabilised. This is most likely due to the steric repulsion of the thiol chains at low Au:thiol ratios. The Au:thiol ratio decided upon for this study was 1:1 as it produced GNPs of the desirable size of $15 \mathrm{~nm}$.

The stability of the peptide capped GNPs is an important factor in the selection of the ideal GNP composite for use in subsequent biological studies. The peptides coupled to the GNPs have an integral effect on the stability of the resultant GNPs as discussed in the following section.

\subsubsection{Colloidal stability determinations for the peptide capped GNPs}

The synthesis of colloidally stable GNPs is vital to circumvent the possibility of irreversible agglomeration of the particles. Peptides are particularly useful as they effectively alter the properties of NPs to yield stable macromolecules. The sequence and length of the peptides attached to the GNPs are a contributing factor to the stability of the resultant GNP composites. ${ }^{\mathbf{8 9 , 9 0}}$ As a result, the effect of the peptide length and sequence on the stability of the GNP composites was examined. 
The aggregation observed in Au-CKCoum, Au-CMECoum and Au-CGAFKGGCoum was not evident in Au-CKAFKRKCoum and Au-C(KAFKRK) $)_{3}$ Coum. For Au-CKCoum and AuCMECoum the length of the thiols was the obvious drawback that led to aggregation, as they appear not to provide sufficient separating distance between the particles. ${ }^{30}$ In the case of the AuCGAFKGGCoum, the glycine residue in the second position is believed to be the main cause of the colloidal instability: Glycine prevents the formation of a dense peptide layer as it hinders the formation of $\beta$-sheet structures hence leading to poor stabilization. ${ }^{\mathbf{8 3 , 9 1}}$

The composites Au-CKAFKRKCoum and Au-C(KAFKRK) $)_{3}$ Coum were further investigated due to their colloidal stability. To test the extent of dispersity of the colloids in water, the absorbance of various concentrations of the GNP solutions was measured. It was found that for both GNP solutions the absorbance increases linearly with increasing concentration and the absorption maximum $\left(\lambda_{\max }\right)$ does not change with concentration (see Figure 35 and Figure 36). This spectral feature suggests that Au-CKAFKRKCoum and Au-C(KAFKRK $)_{3}$ Coum are well dispersed in water.

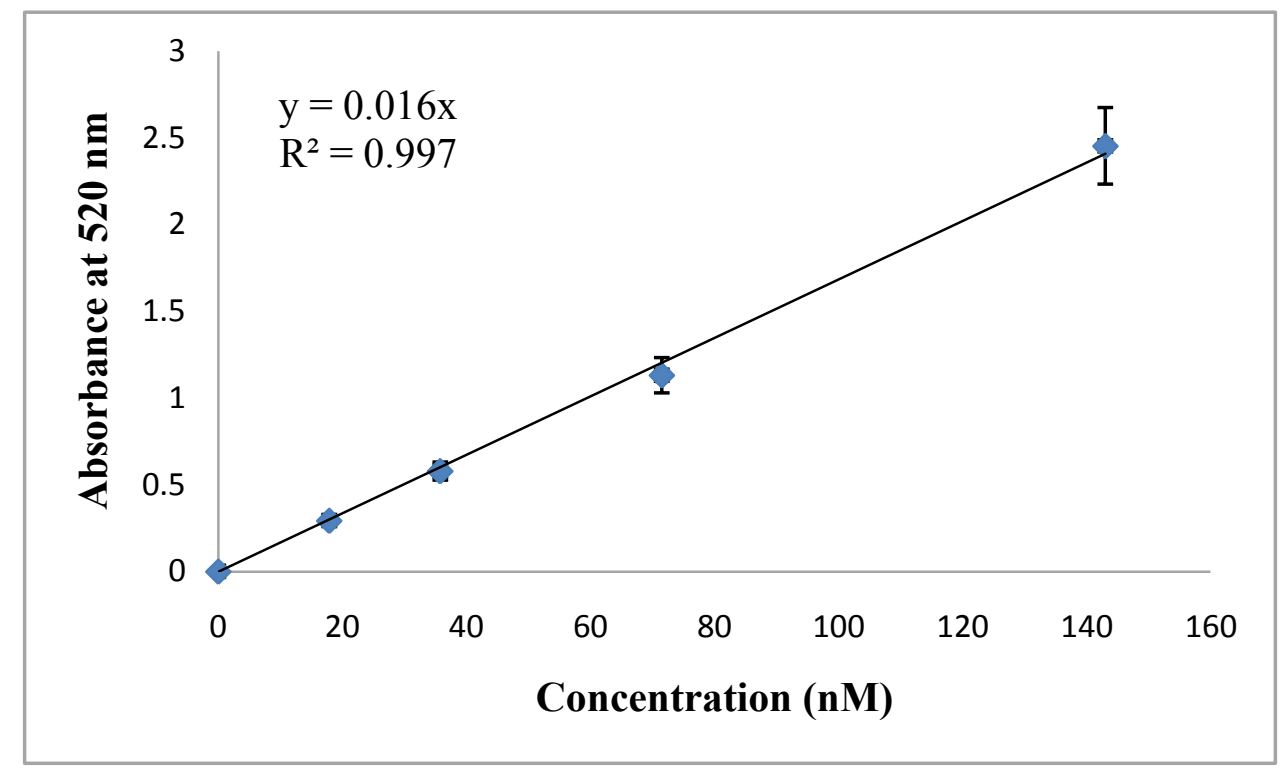

Figure 35: Plot of absorbance of Au-CKAFKRKCoum at $520 \mathrm{~nm}$ as a function of concentration. Each data point is representative of a total of three assays. 


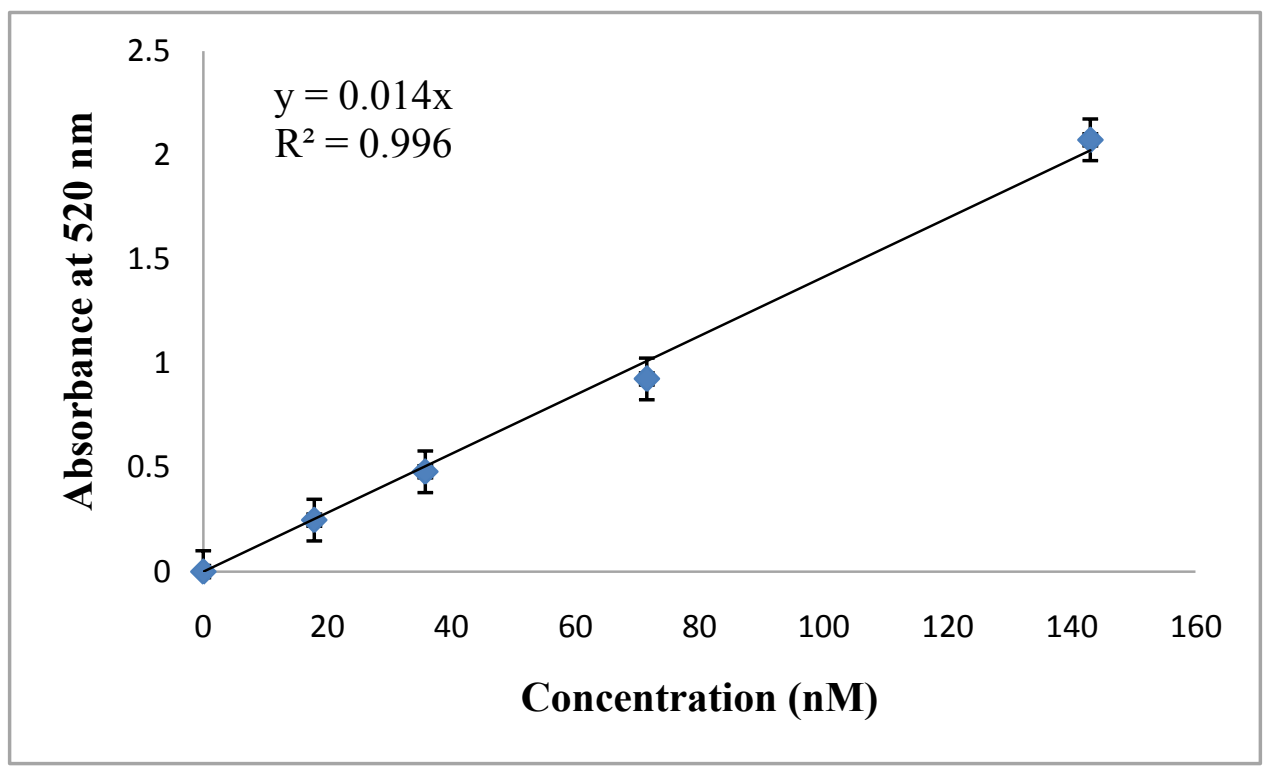

Figure 36: Plot of absorbance of Au-C(KAFKRK $)_{3}$ Coum at $520 \mathrm{~nm}$ as a function of concentration. Each data point is representative of a total of three assays.

Another useful test for the colloidal stability of the particles is the calculation of the critical coagulation concentration (CCC). The CCC is described as the point at which rapid coagulation of the particles occurs. It is determined by the concentration of electrolyte (sodium chloride $-\mathrm{NaCl}$ ) needed to shield the particlese respulsive influence from each other to the extent that there remains no repulsive effect between the particles, thereby maximising the attractive forces. ${ }^{\mathbf{9}}$

Aggregation was assessed by monitoring changes in the characteristic GNP plasmon frequency. $\mathrm{NaCl}$ was added to the solution until the plasmon band was significantly diminished. For Au$\mathrm{C}(\text { KAFKRK })_{3}$ Coum $\left(1.7 \times 10^{-10} \mathrm{M} ; 5 \mathrm{~mL}\right)$, no visible aggregation was observed and the plasmon band did not shift to a longer wavelength (see Figure 38) indicating that the particles were stable up to a $7 \mathrm{~mL}$ addition of a $1.7 \mathrm{M} \mathrm{NaCl}$ solution. For Au-CKAFKRKCoum $\left(1.7 \times 10^{-10} \mathrm{M} ; 5 \mathrm{~mL}\right)$, aggregation was observed after the addition of $4 \mathrm{~mL} \mathrm{NaCl}$ solution as can be seen from the decrease of the absorbance band at $520 \mathrm{~nm}$ and the increase in absorption at longer wavelengths (see Figure $37)$.

The results obtained illustrate the effect of the length of the peptide sequences on the stability of the GNPs. It is evident that the CKAFKRKCoum peptide does not provide the required colloidal stability to the GNP composite necessary to prevent aggregation of the particles and is thus impractical for use in biological systems. The longer version $\mathrm{C}(\mathrm{KAFKRK})_{3}$ must be attached to the GNPs to provide stability. Stability tests were also carried out on Au-10\% CKAFKRKCoum:40\% CKAFKRK:50\% C(KAFKRK $)_{3}\left(1.7 \times 10^{-10} \mathrm{M} ; 5 \mathrm{~mL}\right)$ and no visible aggregation was observed and 
the plasmon band did not shift to longer wavelengths upon addition of $\mathrm{NaCl}$ (Figure 39) thus indicating the particles were stable up to a $7 \mathrm{~mL}$ addition of a $1.7 \mathrm{M} \mathrm{NaCl}$ solution.

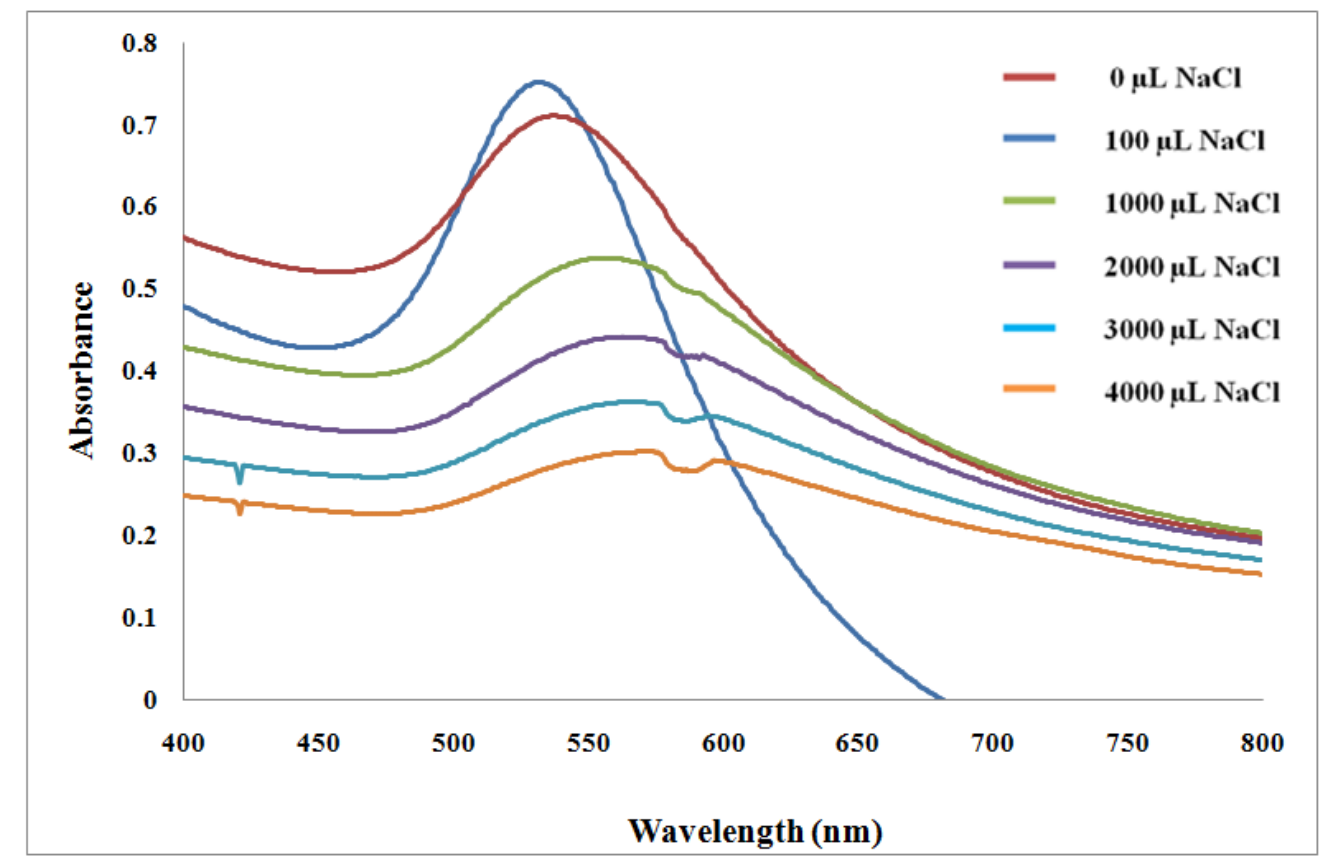

Figure 37: Stability of Au-CKAFKRKCoum $\left(1.7 \times 10^{-10} \mathrm{M} ; 5 \mathrm{~mL}\right)$ assessed by measuring the critical coaguation concentration using $1.7 \mathrm{M} \mathrm{NaCl}$ solution.

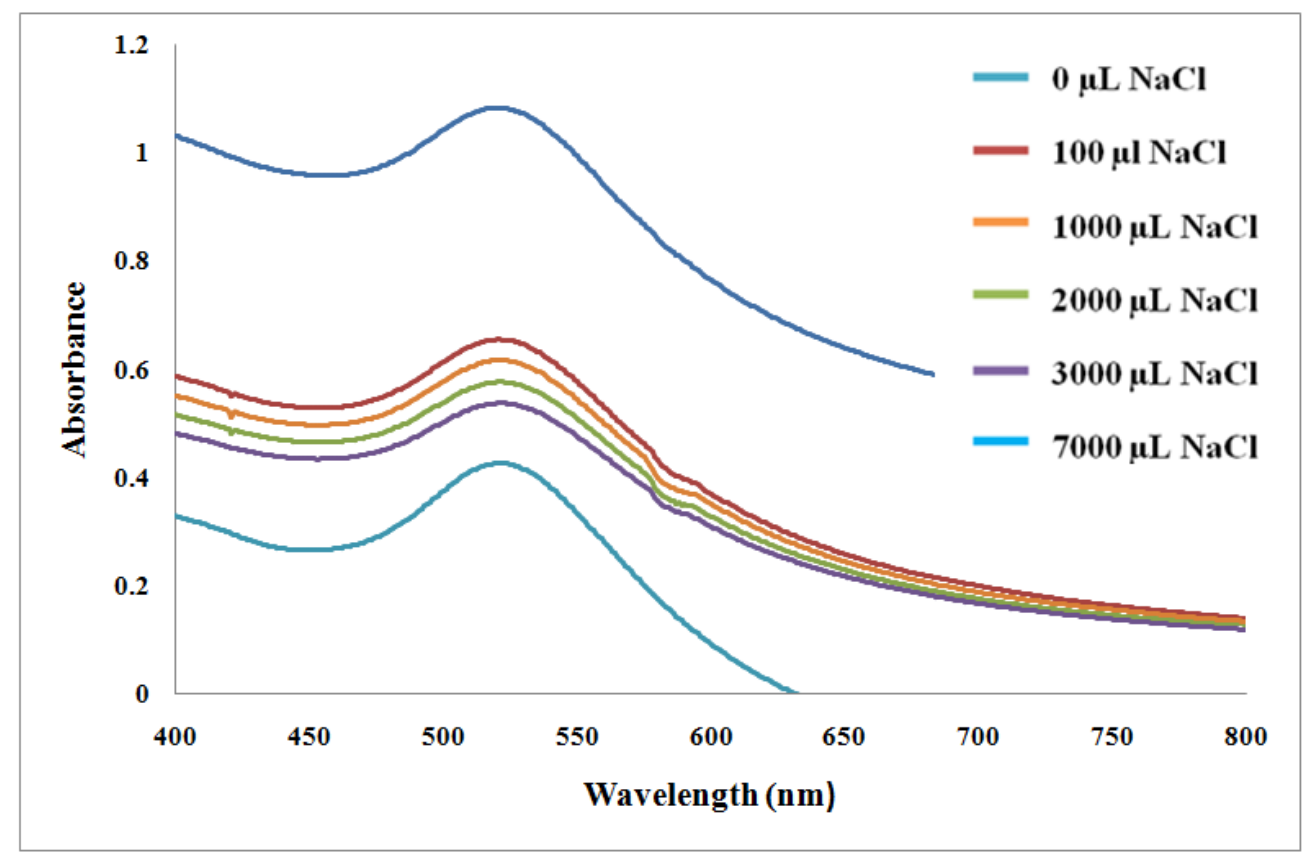

Figure 38: Stability of Au-C(KAFKRK $)_{3}$ Coum $\left(1.7 \times 10^{-10} \mathrm{M} ; 5 \mathrm{~mL}\right)$ assessed by measuring the critical coagulation concentration using $1.7 \mathrm{M} \mathrm{NaCl}$ solution. 


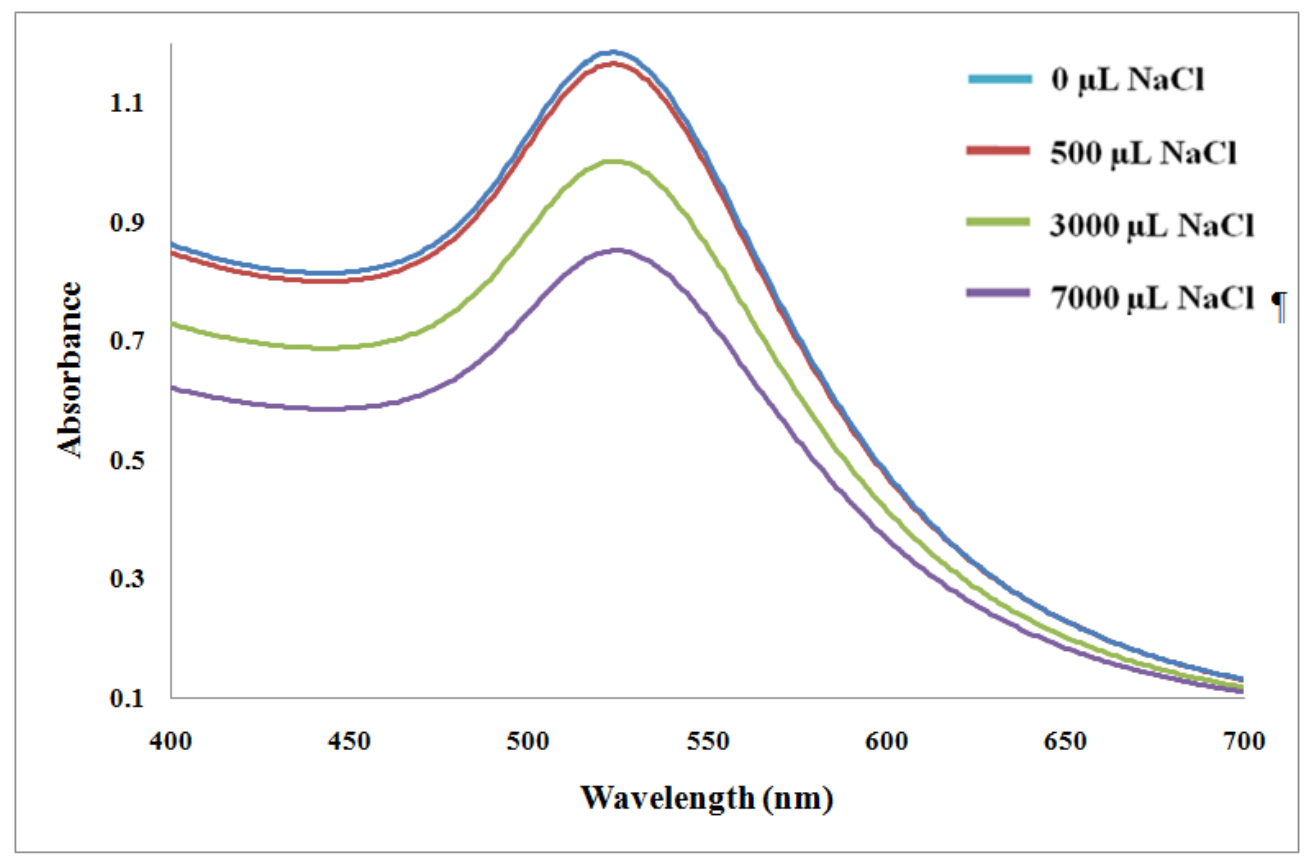

Figure 39: Stability of Au-10\% CKAFKRKCoum: $40 \%$ CKAFKRK:50\% C(KAFKRK) ${ }_{3}\left(1.7 \times 10^{-}\right.$ ${ }^{10} \mathrm{M} ; 5 \mathrm{~mL}$ ) assessed by measuring the critical coagulation concentration using $1.7 \mathrm{M} \mathrm{NaCl}$ solution.

Once the synthetic methodology of the functionalised GNPs was established, various techniques were necessary to optimise and test the functionalised GNPs (described in detail in Chapter 5: Testing and optimisation of peptide capped 


\section{CHAPTER 5: TESTING AND OPTIMISATION OF PEPTIDE CAPPED GNPS}

Various techniques such as fluorescence spectroscopy, trypsin digestion, mass spectrometry, cell viability testing, transmission electron microscopy, and confocal microscopy were utilised in an attempt to optimize and test the GNPs for proof of concept. The techniques used are described briefly in section 5.1. to 5.5. (pages 65 to 71 ).

\subsection{FLUORESCENCE SPECTROSCOPY}

Fluorescence spectroscopy is a type of electromagnetic spectroscopy which analyses fluorescence from a sample. It is a well-established analytical method that is widely used in the fields of biochemistry and molecular biology as a minimally invasive method. The technique has grown popular because of its acute sensitivity to changes in the structural and dynamic properties of biomolecules and biomolecular complexes. ${ }^{93}$

\subsubsection{The process of fluorescence}

Fluorescence is a photon emission process that occurs during molecular relaxation from electronic excited states. $^{94}$ When a molecule is excited from the ground state $\left(\mathrm{S}_{0}\right)$ by absorbing electromagnetic radiation (a photon) of some unique energy supplied by an external source (incandescent lamp or laser) an excited electronic singlet state $\left(\mathrm{S}_{1}\right)$ is reached. ${ }^{\mathbf{9 5 , 9 6}}$ The molecule will eventually attempt to lower this higher than normal energy state. There are several mechanisms by which the molecule can relax to its original ground state. These relaxation processes may be radiative or luminescent in nature or may occur through radiationless processes. ${ }^{\mathbf{9 7}}$ Radiationless processes include vibrational relaxation, external conversion, internal conversion and intersystem crossing. ${ }^{96}$ Luminescent processes include phosphorescence and fluorescence. There are two fundamental differences between phosphorescence and fluorescence: The former occurs when the excited state is of a different electronic spin state than the ground state whereas the latter occurs when the excited state is of the same electronic spin state as the ground state. ${ }^{\mathbf{9 6}}$ The Jablonski diagram (Figure 40) offers a convenient representation of the excited state structure and the relevant transitions. 


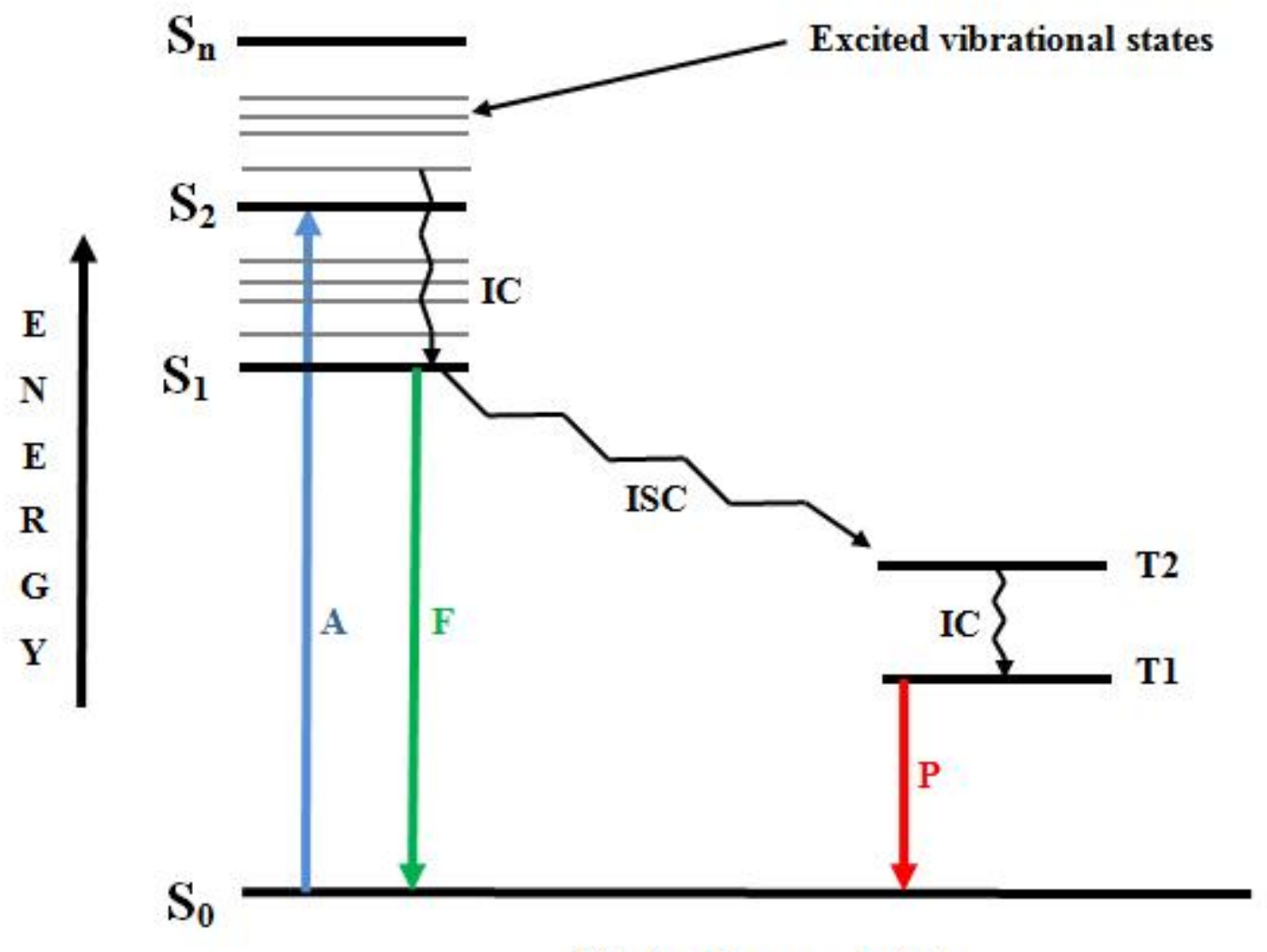

Electronic ground state

Where: $\quad$ A $=$ Photon absorption

$$
\begin{aligned}
& \mathbf{F}=\text { Fluorescence } \\
& \mathbf{P}=\text { Phosphorescence } \\
& \mathbf{S}=\text { Singlet state } \\
& \mathbf{T}=\text { Triplet state } \\
& \text { IC = Internal conversion } \\
& \text { ISC = Intersystem crossing }
\end{aligned}
$$

Figure 40: Jablonski diagram. Redrawn from Thomas Chasteen (1996). ${ }^{\mathbf{9 8}}$

An interesting phenomenon of fluorescence that may occur between two fluorophores in close proximity is referred to as fluorescence resonance energy transfer and is explained further in the following section. 


\subsubsection{Fluorescence resonance energy transfer}

When the separation distance between two fluorescent chromophores ${ }^{\mathrm{i}}$ is decreased a phenomenon known as fluorescence resonance energy transfer (FRET) occurs. ${ }^{\mathbf{9 5 , 9 9 , 1 0 0}}$ FRET is defined as a distance-dependent interaction between the electronic excited states of two fluorophores involving the radiationless transfer of energy from the donor fluorophore to the acceptor fluorophore. ${ }^{\mathbf{1 0 0}}$ The primary conditions for FRET are listed below:

- Donor and acceptor molecule must be in close proximity (1-10 nm),

- the absorption spectrum of the acceptor must overlap the fluorescence emission spectrum of the donor (Figure 40),

- donor and acceptor transition dipole orientations must be approximately parallel. ${ }^{\mathbf{1 0 0}}$

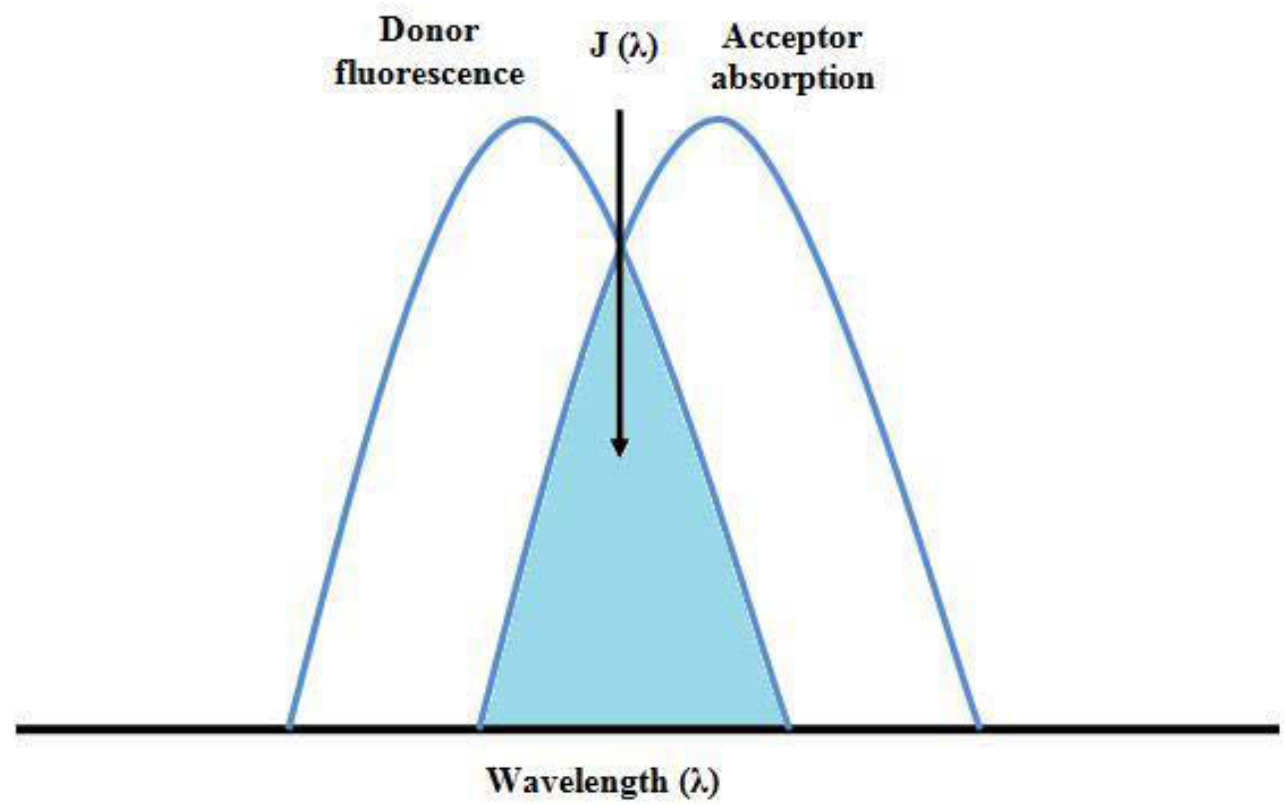

Figure 41: Schematic representation of the FRET spectral overlap integral. ${ }^{\mathbf{1 0 0}}$

Fluorophores in close proximity to GNPs fluoresce weakly due to the occurrence of FRET between the fluorophore and GNP. Of all the metal NPs, GNPs in particular are known to be efficient quenchers for most fluorophores due to their high molar absorbtivities in the visible region. ${ }^{37}$ For experimental purposes it was essential that the fluorescence of Coumarin-3-carboxylic acid not be

\footnotetext{
${ }^{\mathrm{i}}$ A chemical group capable of selective light absorption resulting in the colouration of certain organic compounds.
} 
quenched by the gold. Fluorescence analyses were carried out to ensure that FRET was not active between gold and Coumarin (refer to section 5.6.1., page 75 and section 5.6.3., page 77, for results).

As mentioned in section 1.3. (page 5) it was anticipated that digestion of the ,forest of long peptides $^{\text {ee }}$ should ideally expose the shorter peptide, thereby exposing greater fluorescence intensity. This hypothesis was tested by trypsin digestion of the peptide capped GNPs to assess the affect of cleavage on the fluorescence intensity (section 5.6.6., page 84).

\subsection{TRYPSIN DIGESTION OF PEPTIDE CAPPPED GNPS}

Trypsin is a serine protease found in the digestive system of many vertebrates where it hydrolyses (breaks down) proteins into their constituent amino acids. The protease digests proteins (polypeptides) by cleaving the peptide chain at specific sites. Trypsin cleaves the peptide chain after lysine or arginine residues. Generally, tryptic digestion leaves behind the protein as peptide fragments consisting of none or one lysine or arginine residue. ${ }^{101}$ This property of trypsin is widely used to study the primary structure and identification of proteins by analysing the resultant peptides using mass spectrometry (MS) (section 5.3., page 69).

A number of references concerning the cleavage of the groups attached to the surface of GNPs (on exposure to proteases) are recorded in the literature. Guarise et al. ${ }^{\mathbf{1 0 2}}$ developed a colourimetric assay that allows for the detection of a protease, based on the cleavage of thiol groups attached to the surface of GNPs. Lee et al. ${ }^{103}$ developed a near IR fluorescence assay based on the cleavage of a substrate attached to GNPs and Zhen et al. ${ }^{\mathbf{1 0 4}}$ made use of the ability of thrombin to cleave a peptide attached to GNPs.

It was hypothesized that the length of the peptide attached to the GNP surface would affect the ease of digestion by the protease: A $100 \% \mathrm{C}(\mathrm{KAFKRK})_{3}$ functionalised GNP solution is likely to produce greater hindrance of the process of digestion than a 50:50\% C(KAFKRK) 3 :CKAFKRK functionalised GNP solution. Such an investigation has not previously been reported, hence a preliminary study was conducted to investigate this. The peptide $C(\text { KAFKRK })_{3}$ was digested first to determine the peptide fragments resulting from cleavage. The fragments were analysed by mass spectrometry (MS) and the mass and retention time of the fragments were determined. The peptide capped GNPs were then digested and the cleavage products tested by MS (section 5.6.3., page 77). 


\subsection{MASS SPECTROMETRY}

Mass spectrometry (MS) is a powerful analytical technique used for identifying unknown compounds, to quantify known materials, and to elucidate the structure and chemical properties of molecules. ${ }^{12}$ The MS principle consists of ionising chemical compounds to generate electrically charged molecules or ions. ${ }^{105}$ The motion of the ion in an electric/ magnetic field allows for the determination of the mass to charge ratio $(\mathrm{m} / \mathrm{z})$ of the ion. ${ }^{105,106}$ In a typical MS procedure:

- The sample is loaded onto the MS instrument,

- the components of the sample are ionised resulting in the formation of charged particles,

- the ions are directed into a magnetic/electric field,

- the $\mathrm{m} / \mathrm{z}$ ratio of the particles is elucidated by the motion of the ions as they transit through electromagnetic fields,

- detection of the ions. ${ }^{105}$

The MS instrument consists of three main modules: An ion source, which can convert gas phase molecules into charged fragments, a mass analyzer that sorts the ions by their masses by the application of electromagnetic fields, and a detector, which calculates the abundance of each ion present (Figure 42). ${ }^{\mathbf{1 0 5 , 1 0 7}}$

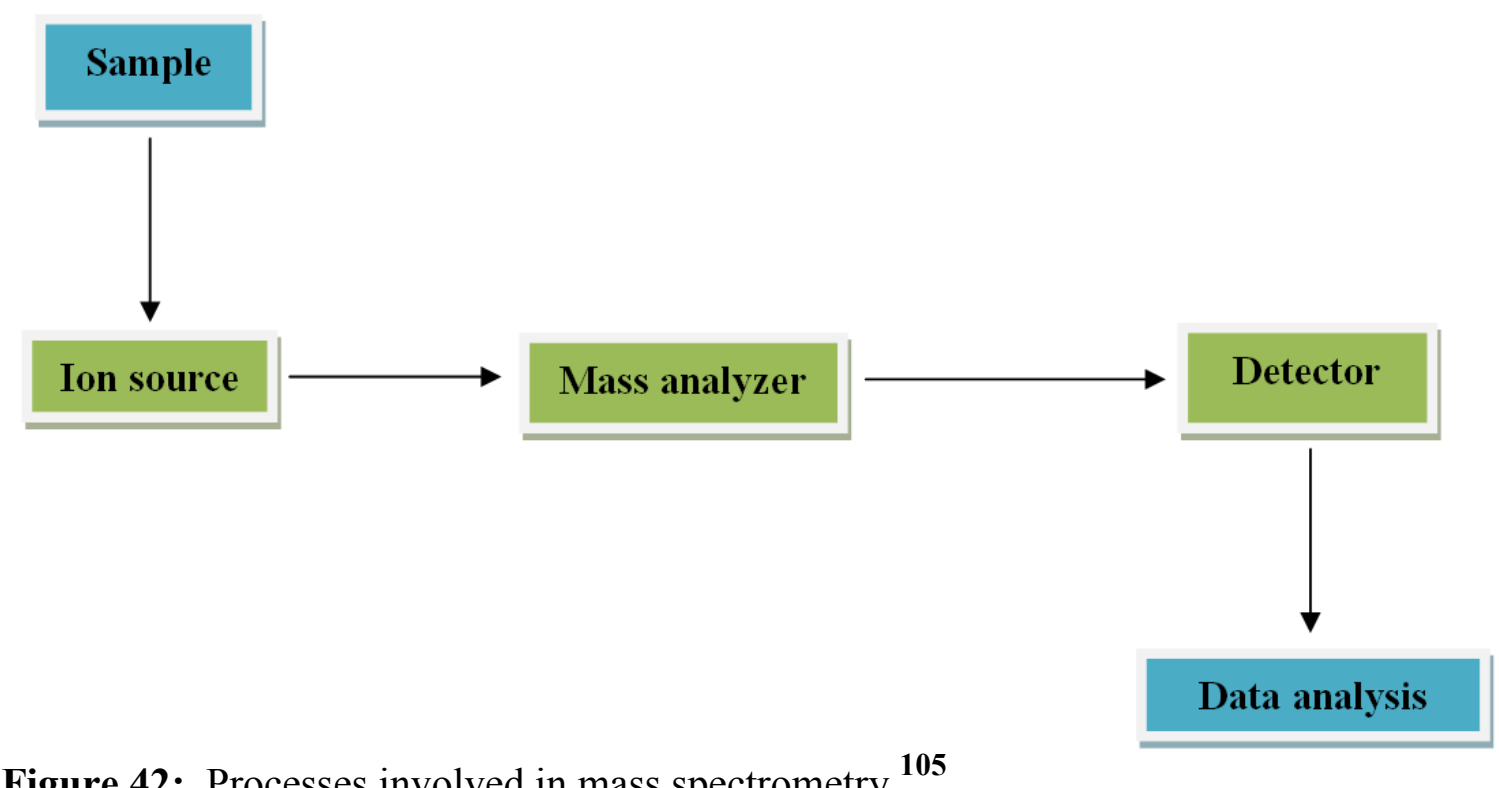

Figure 42: Processes involved in mass spectrometry. ${ }^{105}$

There are different methods of ionisation and the choice depends on the nature of the sample and the type of information required from the analysis (Table 8). ${ }^{\mathbf{1 0 7}}$ Mass analysers sort the ions based 
on their $\mathrm{m} / \mathrm{z}$ ratio and various types can be used depending on the resolution required for the analysis.

Table 9: Different types of ionization methods used in mass spectrometry. ${ }^{107}$

\begin{tabular}{|c|c|c|c|c|}
\hline $\begin{array}{l}\text { Ionisation } \\
\text { method }\end{array}$ & Typical analytes & $\begin{array}{c}\text { Sample } \\
\text { introduction }\end{array}$ & Mass range & $\begin{array}{l}\text { Method } \\
\text { highlights }\end{array}$ \\
\hline $\begin{array}{c}\text { Electron } \\
\text { impact }(\mathbf{E I}) .\end{array}$ & $\begin{array}{c}\text { Relatively small, } \\
\text { volatile. }\end{array}$ & $\begin{array}{c}\text { GC or liquid/solid } \\
\text { probe. }\end{array}$ & $\begin{array}{l}\text { To } 1000 \\
\text { Daltons. }\end{array}$ & $\begin{array}{l}\text { Hard method, } \\
\text { versatile, provides } \\
\text { structural info. }\end{array}$ \\
\hline $\begin{array}{l}\text { Chemical } \\
\text { ionisation } \\
\text { (CI). }\end{array}$ & $\begin{array}{c}\text { Relatively small, } \\
\text { volatile. }\end{array}$ & $\begin{array}{c}\text { GC or liquid/solid } \\
\text { probe. }\end{array}$ & $\begin{array}{l}\text { To } 1000 \\
\text { Daltons. }\end{array}$ & $\begin{array}{c}\text { Soft method, } \\
\text { molecular ion peak } \\
{\left[\mathrm{M}+\mathrm{H}^{+}\right] .}\end{array}$ \\
\hline $\begin{array}{c}\text { Electrospray } \\
\text { ionisation } \\
\text { (ESI). }\end{array}$ & $\begin{array}{l}\text { Peptides, proteins, } \\
\text { non-volatile. }\end{array}$ & $\begin{array}{l}\text { Liquid } \\
\text { chromatography or } \\
\text { syringe. }\end{array}$ & $\begin{array}{l}\text { To } 200000 \\
\text { Daltons. }\end{array}$ & $\begin{array}{l}\text { Soft method, ions } \\
\text { often multiply } \\
\text { charged. }\end{array}$ \\
\hline $\begin{array}{c}\text { Fast atom } \\
\text { bombardment } \\
\text { (FAB). }\end{array}$ & $\begin{array}{c}\text { Carbohydrates, } \\
\text { organometallics, } \\
\text { peptides, non-volatile }\end{array}$ & $\begin{array}{l}\text { Sample mixed in } \\
\text { viscous matrix. }\end{array}$ & $\begin{array}{l}\text { To } 6000 \\
\text { Daltons. }\end{array}$ & $\begin{array}{l}\text { Soft method but } \\
\text { harder than ESI or } \\
\text { MALDI. }\end{array}$ \\
\hline $\begin{array}{c}\text { Matrix } \\
\text { assisted laser } \\
\text { desorption } \\
\text { (MALDI). }\end{array}$ & $\begin{array}{c}\text { Peptides, proteins, } \\
\text { nucleotides. }\end{array}$ & $\begin{array}{l}\text { Sample mixed in } \\
\text { solid matrix. }\end{array}$ & $\begin{array}{l}\text { To } 500000 \\
\text { Daltons. }\end{array}$ & $\begin{array}{l}\text { Soft method, very } \\
\text { high mass. }\end{array}$ \\
\hline
\end{tabular}

\section{Mass spectrometric analysis of peptides}

The structural characterization of peptides is an integral aspect of biotechnology/pharmaceutical research in the development of novel therapeutic agents. ${ }^{108}$ With the advent of new and advanced desorption techniques in MS, peptide molecules can now be easily ionised in the MS instrument without prior derivitisation. The three most common MS methods used today for protein molecular weight determinations are ESI-MS, MALDI MS and plasma desorption MS. ${ }^{109}$ As ESI-MS has online liquid chromatography (LC) MS capabilities, and was the technique of choice for the analysis of the various synthetic peptides in this project. ${ }^{109}$ 


\section{Mass spectrometric analysis of functionalised GNPs}

GNPs have been widely used as inorganic matrices for MALDI-Time of flight (TOF) MS detection of biomeolecules, peptides, amino acids and low molecular weight polymers. ${ }^{\mathbf{1 1 0 - 1 1 2}}$ Despite this, there are very few references in the literature on the mass spectral analysis of the surface chemistry of GNPs. Of these references MALDI-TOF MS is primarily used, due to the lack of solubility of the functionalised GNPs in most solvents. ${ }^{113,114}$ Soluble GNPs allow for the use of alternate MS techniques, such as ESI-MS: Gies et al. $(2007)^{\mathbf{1 1 5}}$ employed ESI-MS for the investigation of tiopronin monolayer protected GNPs.

Since the final GNPs synthesised in this project were completely water soluble, ESI-MS was the method of choice for the analysis of the peptide capped GNPs as well as the fragments that resulted from trypsin digestion (refer to section 5.6.3., page 77, for results).

\subsection{CYTOTOXICTY TESTING}

Cytotoxicty testing is a technique that enables one to establish whether a chemical agent is able to adversely affect the metabolism of a cell resulting in the inhibition of cell growth. This test is widely used in the field of drug development as it provides a crucial means of ranking compounds for consideration in drug discovery. ${ }^{116-118}$

A number of cytotoxic test systems have been developed with the intention of producing results interrelated with in vivo efficacy studies. ${ }^{119}$ The tests differ in parameters associated with cell death to give a clear indication of the toxicity of any sample. The most convenient modern assays have been optimised for use of microtitre plates (96-well format), allowing samples to be analyzed rapidly and simultaneously with simple colourimetric and luminescence based detection systems. ${ }^{\mathbf{1 2 0}}$ The four most commonly methods used are

- The lactate dehydrogenase $(\mathrm{LDH})^{\mathrm{j}}$ assay,

- the 3-(4,5-dimethylthiazol-2-yl)-2,5-diphenyltetrazolium bromide (MTT) assay,

- the natural red (3-amino-m-dimethylamino-2-methylphenazinehydrochloride) assay,

- the adenosine triphosphate (ATP) ${ }^{\mathrm{k}}$ assay. $^{119}$

\footnotetext{
${ }^{\mathrm{j}} \mathrm{LDH}$ is an enzyme which catalyses the conversion of pyrate in the absence of oxygen and thus yields energy for cellular processes.
} 
Since a number of methods have been developed to study cell viability and proliferation in cell culture [Cook and Mitchell, (1989)], ${ }^{\mathbf{1 2 1}}$ it was difficult to decide on an appropriate assay. ${ }^{\mathbf{1 2 0}}$ Research indicates that the most common method used for the study of toxicity of GNPs to cells is the MTT assay and it was thus the method of choice for this project.

The MTT assay is a colourimetric technique that focuses on the metabolic activity of viable cell mitochondria as an indication of toxicity. Only metabolically active cells are able to reduce MTT, a water soluble tetrazolium salt, to an insoluble blue-violet formazan crystal by cleavage of the tetrazolium ring by succinate dehydrogenase within the mitochondria. The formazan product is impermeable to the cell membranes and therefore accumulates in healthy cells (see Figure 43) [Mosmann, (1983)]. ${ }^{\mathbf{1 2 0 , 1 1 9}}$

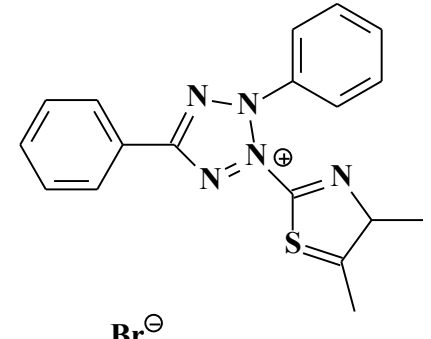

$\mathrm{Br}^{\ominus}$

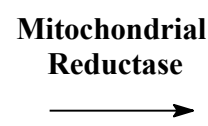

Formazan

Figure 43: Mechanism of MTT reduction to produce formazan crystals. Redrawn from Rode et al. (2007). ${ }^{122}$

The human lung epithelium cell line (A549) ${ }^{1}$ and the Madin Darby bovine kidney epithelium $(\mathrm{MDBK})^{\mathrm{m}}$ cells are widely used in MTT assays involving GNP research and were thus used for cytotoxicity testing in this project. The A549 and MDBK cell line was donated by colleagues in the school of Biochemistry at the University of KwaZulu-Natal. Both A549 and MDBK are adherent ${ }^{\mathrm{n}}$ and were suitable for the MTT assay in 96-well plates (refer to section 5.6.9., page 89, for the MTT results).

\footnotetext{
${ }^{\mathrm{k}}$ ATP is a chemical compound present in living organisms that releases energy for use in cellular processes.

${ }^{1}$ A549 cells are cancerous epithelial cells and were isolated from human lung tissue.

${ }^{m}$ The MDBK cell line is a non-cancerous epithelial cell line that was isolated from the kidney of a cow.

${ }^{\mathrm{n}}$ Adherent cells grow by attaching to the surface of the culture vessel and are not washed off during washing or media change.
} 


\subsection{MICROSCOPY}

Microscopy is the scientific discipline which involves magnifying objects to the extent that features which cannot be seen with the naked eye become visible. The three well-known branches of microscopy are optical, electron and scanning probe microscopy. ${ }^{123}$ The first two methods will be discussed further since they were utilised in this project.

\subsubsection{Optical microscopy}

Optical microscopy was the first form of microscopy to be introduced and involves passing visible light transmitted through or reflected from the sample through single or multiple lenses to allow a magnified view of the sample. ${ }^{\mathbf{1 2 3 , 1 2 4}}$ Fluorescence microscopy is a type of optical technique that is widely used. $^{125}$ In fluorescence microscopy, light of a particular wavelength is passed through a specialised microscope condenser which focuses the light into a narrow beam. ${ }^{\mathbf{1 2 5}}$ This light strikes the specimen and molecules are excited, resulting in the emission of light at a lower frequency. ${ }^{\mathbf{1 2 5}}$

In a conventional wide-field fluorescence microscope, the entire spectrum is flooded with light from the light source. ${ }^{125}$ All parts of the sample in the optical path are illuminated by the excitation light and thus the entire sample fluoresces simultaneously. ${ }^{\mathbf{1 2 3 , 9 5}}$ Despite the highest intensity of excitation light being at a focal point of the lens, the other areas of the sample are illuminated, creating a background haze in the resulting image. ${ }^{\mathbf{1 2 5}}$ In 1957, Marvin Minsky overcame the problem of conventional wide-field fluorescence microscopy by employing point illumination and a pinhole in front of the detector to eliminate out-of-focus information, a technique presently known as confocal fluorescence laser scanning microscopy (CFLSM). ${ }^{\mathbf{1 2 3 , 1 2 6}}$ 
CFLSM offers several advantages over wide-field fluorescence microscopy, such as the:

- Ability to control depth of field,

- elimination or reduction of background information away from the focal plane,

- capability to collect serial optical sections from thick specimens. ${ }^{\mathbf{1 2 3}}$

This technique is therefore extremely suitable for the detection of fluorescent molecules inside living cells. Due to these advantages of CFLSM it was considered a vital analytical tool in this project (refer to section 5.6.10., page 101).

\subsubsection{Electron microscopy}

The electron microscope utilises a beam of highly energetic electrons to examine objects on a very small scale. The technique acquires information about the topography (surface features), morphology (shape and size), composition (chemical identity) and crystallography of the sample in question. $^{\mathbf{1 2 7 , 3 6}}$ Electron microscopes achieve resolutions down to $0.2 \mathrm{~nm}$ and can obtain magnification of up to 300000 times. $^{\mathbf{1 2 8}}$ There are two main types of electron microscopes, namely the scanning electron microscope (SEM) and the transmission electron microscope (TEM). ${ }^{\mathbf{1 2 8}}$ Since the latter technique was used in this project it will be discussed further.

In TEM, a beam of electrons is transmitted through ultrathin specimens, interacting with the specimen as it passes through. ${ }^{\mathbf{1 2 9}}$ After passing through the specimen, the electrons strike a fluorescent screen thereby producing an image. ${ }^{129}$ Sample preparation in TEM can be a complex procedure. TEM specimens are required to be very thin (at most hundreds of nanometres thick) small (no more than $50 \mathrm{~mm}^{2}$ ) and electron dense. ${ }^{129,128}$ GNPs are easily visualised under the TEM due to their small size and electron density and thus TEM was the method of choice to determine the diameter and shape of the GNPs synthesised in this project (refer to section 5.6.7., page 86, for the TEM results). 


\subsection{RESULTS OF TESTING AND OPTIMISATION OF PEPTIDE CAPPED GNPS}

A variety of tests were performed to create the ideal peptide-GNP composite for the purposes of this project. The final composite prepared was then tested with a selection of assays and discussed in this section.

\subsubsection{Peptide length investigations}

Aslan et al. (2007) ${ }^{\mathbf{7 5}}$ reported that the ideal separation distance between fluorophores and GNP is greater than $10 \mathrm{~nm}$ to prevent FRET from occurring. To test this hypothesis, an 18 amino acid $\left[\mathrm{C}(\mathrm{KAFKRK})_{3} \text { Coum with an approximate length of } 6.36 \mathrm{~nm}\right]^{0}$ and a six amino acid (CKAFKRKCoum with an approximate length of $2.18 \mathrm{~nm}$ ) peptide sequence was synthesised, and attached to GNPs. Fluorescence spectroscopy analyses indicated that both distances were sufficient to prevent FRET from occurring as there was a significant amount of fluorescence from both GNP composites. This result is in contrast to the required distance between GNP surface and fluorophore of $10 \mathrm{~nm}$ stated by Aslan et al. ${ }^{\mathbf{7 5}}$

On further review, Shang et al. (2007) ${ }^{\mathbf{1 2 6}}$ reported an increase in fluorescence in conjugated polymer-stabilised GNPs upon addition of cysteine to the surface of the GNPs. ${ }^{\mathbf{8 5}}$ Shang theorised that cysteine can modulate the energy transfer between the fluorophore and the gold atoms. To validate this observation, CMECoum was attached to GNPs and the fluorescence measured. The emission intensity of this composite was significant, confirming the hypothesis of Shang.

As expected, the fluorescence intensity of the capped GNPs was directly proportional to the separation distance between the fluorophore and $\mathrm{Au}$ surface (Figure 44). The peptide length decided upon for further use in the project was CKAKFKRCoum, as the fluorescence emission intensity was sufficient for the purposes of this project.

\footnotetext{
${ }^{\circ}$ The HyperChem software was used to measure the length of the peptide sequences.
} 


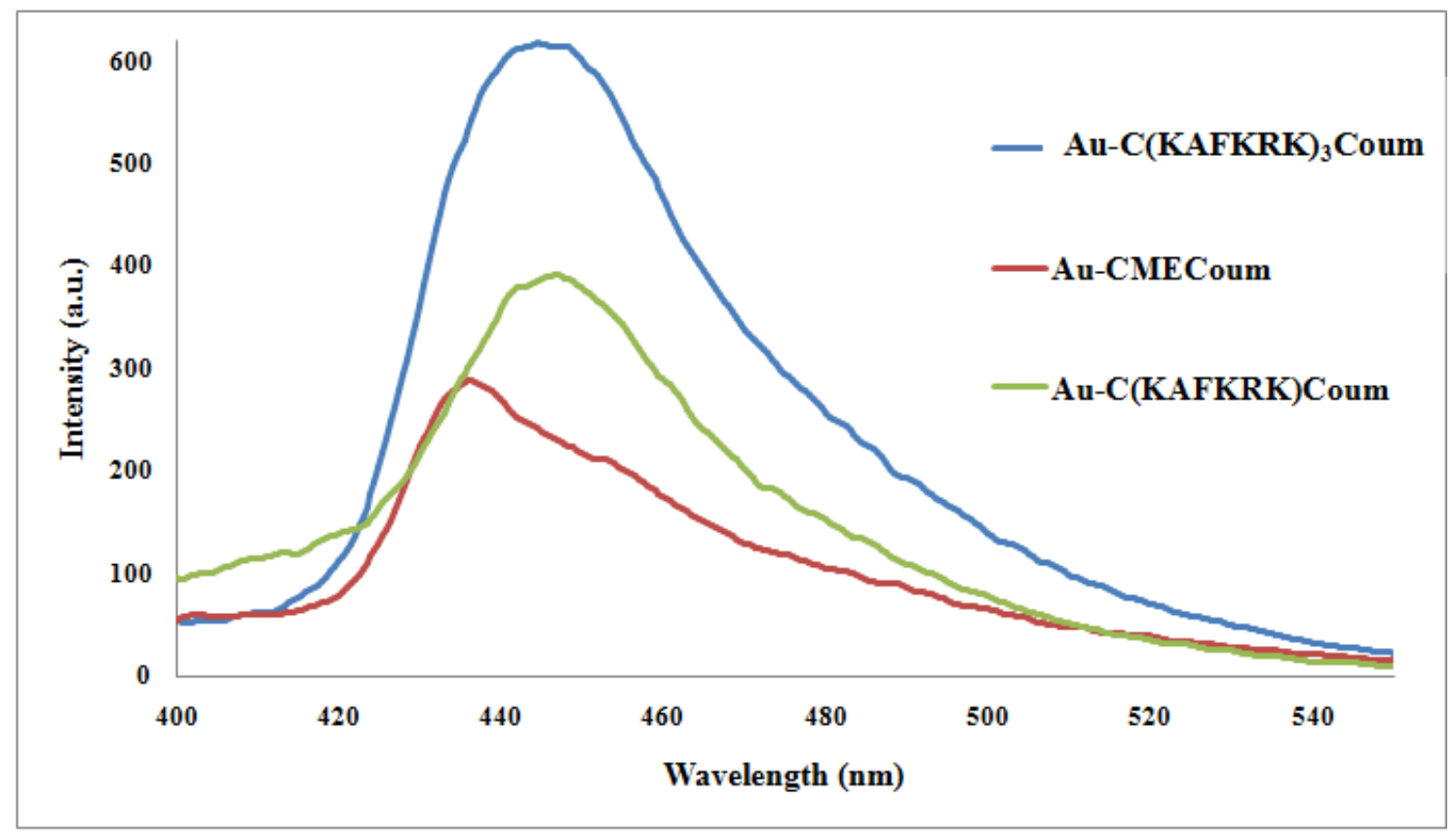

Figure 44: Fluorescence spectra illustrating the difference in emission intensity of various peptide capped GNPs.

\subsubsection{Peptide percentage investigations}

As mentioned previously, the Coumarin moiety will ultimately be exchanged with a radioactive atom at a later stage in the project, the radiation of which should not be too great to cause any toxic effects in patients. Therefore, the percentage of CKAFKRKCoum attached to GNPs should be significantly less than the $\mathrm{C}(\mathrm{KAFKRK})_{3}$ peptide.

Fluorescence tests were done to determine the smallest percentage of CKAKFKRKCoum that could be attached to GNPs and show significant emission intensity. Ratios of 0.1:99.9, 1:99 and 10:90 of CKAFKRKCoum:C(KAFKRK) ${ }_{3}$, respectively, were attached to GNPs. Fluorescence intensity was the lowest in the case of the $0.1 \%$ and the highest in the case of $10 \%$ coverage of the gold surface (Figure 45). It was decided to use the 10\% CKAFKRKCoum as it showed adequate emission intensity. 


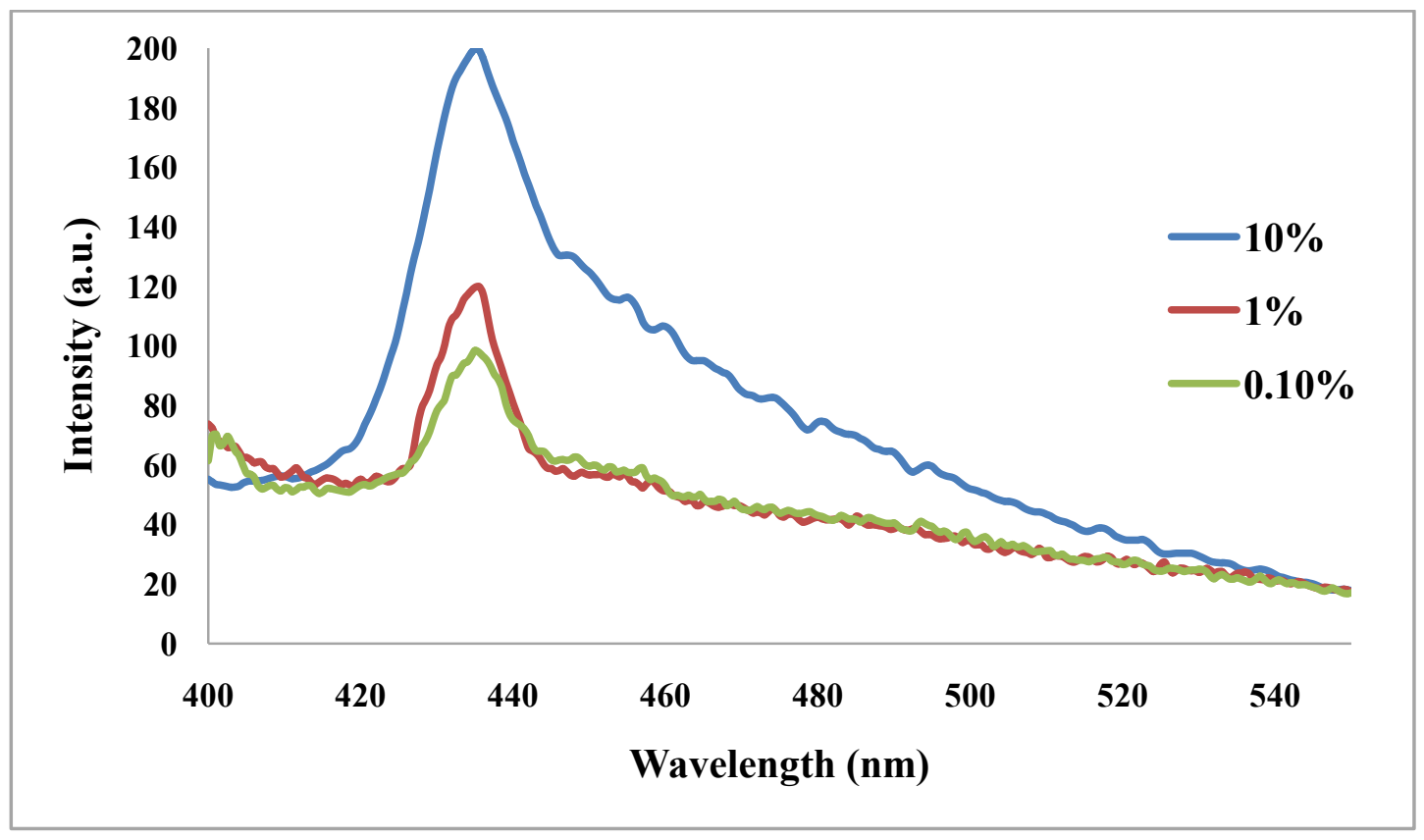

Figure 45: Fluorescence spectra illustrating the difference in emission intensity of various concentrations of CKAFKRKCoum.

\subsubsection{Trypsin digestion of the peptide capped GNPs}

This assay was integral in the determination of the accessibility of the protease to the peptide. Various percentages of $\mathrm{C}(\mathrm{KAFKRK})_{3}$ and CKAFKRK were attached to GNPs, subjected to trypsin digestion and the cleavage products monitored by mass spectral analysis.

A 24 hour test was conducted prior to GNP digestion in order to determine the main cleavage products of the peptide $\mathrm{C}(\mathrm{KAFKRK})_{3}$. Briefly, the experiment was carried out as follows: The peptide was dissolved in water and the appropriate volume of trypsin/versene solution added. The mixture was mixed and left to incubate at $37^{\circ} \mathrm{C}$ for 24 hours, after which the solution was analysed using LC-MS connected to an ESI Q-TOF apparatus.

Interpretation of the spectrum indicated that the main peak at $493 \mathrm{~m} / \mathrm{z}$ could be ascribed to the fragment KAFK. Figure 46 and Figure 47 show the mass spectra of the peptide before and after cleavage respectively. 


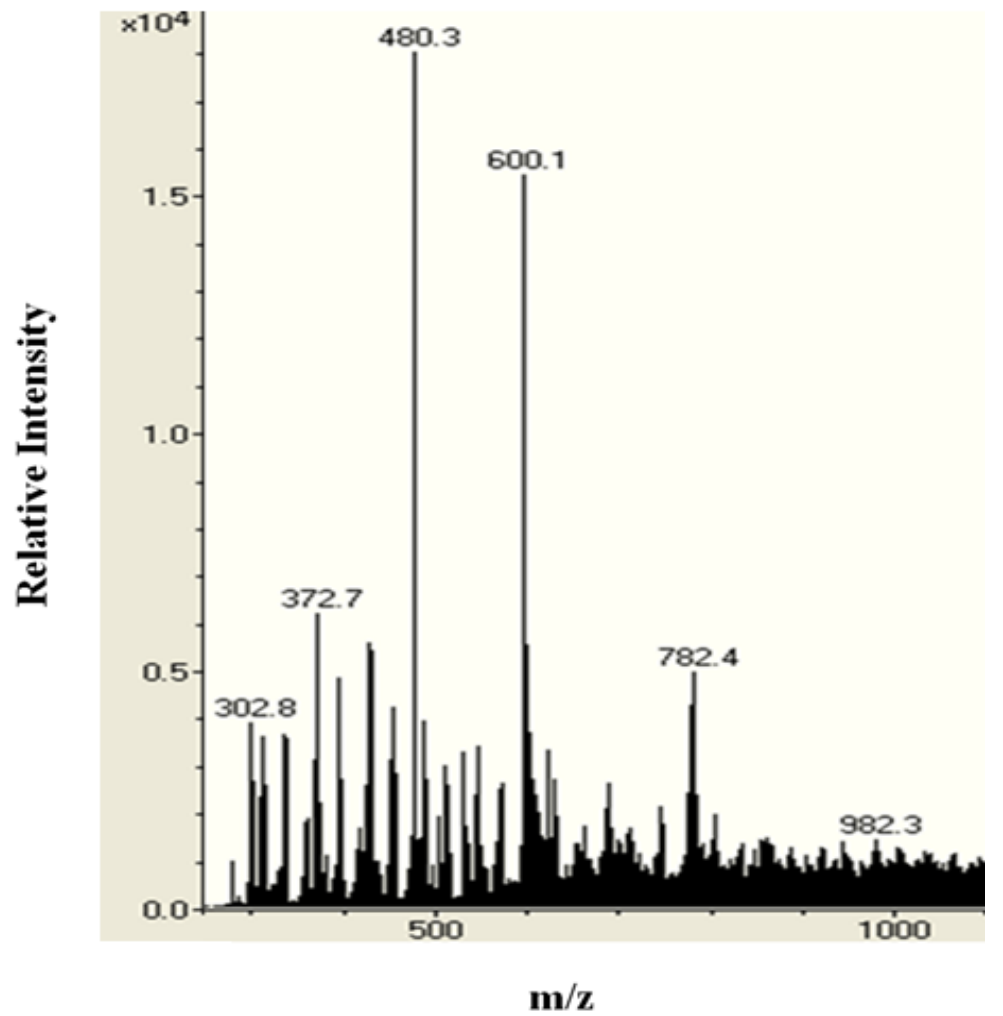

Figure 46: Mass spectrum of $\mathrm{C}(\mathrm{KAFKRK})_{3}$ in phosphate buffered saline (PBS) prior to enzymatic degradation.

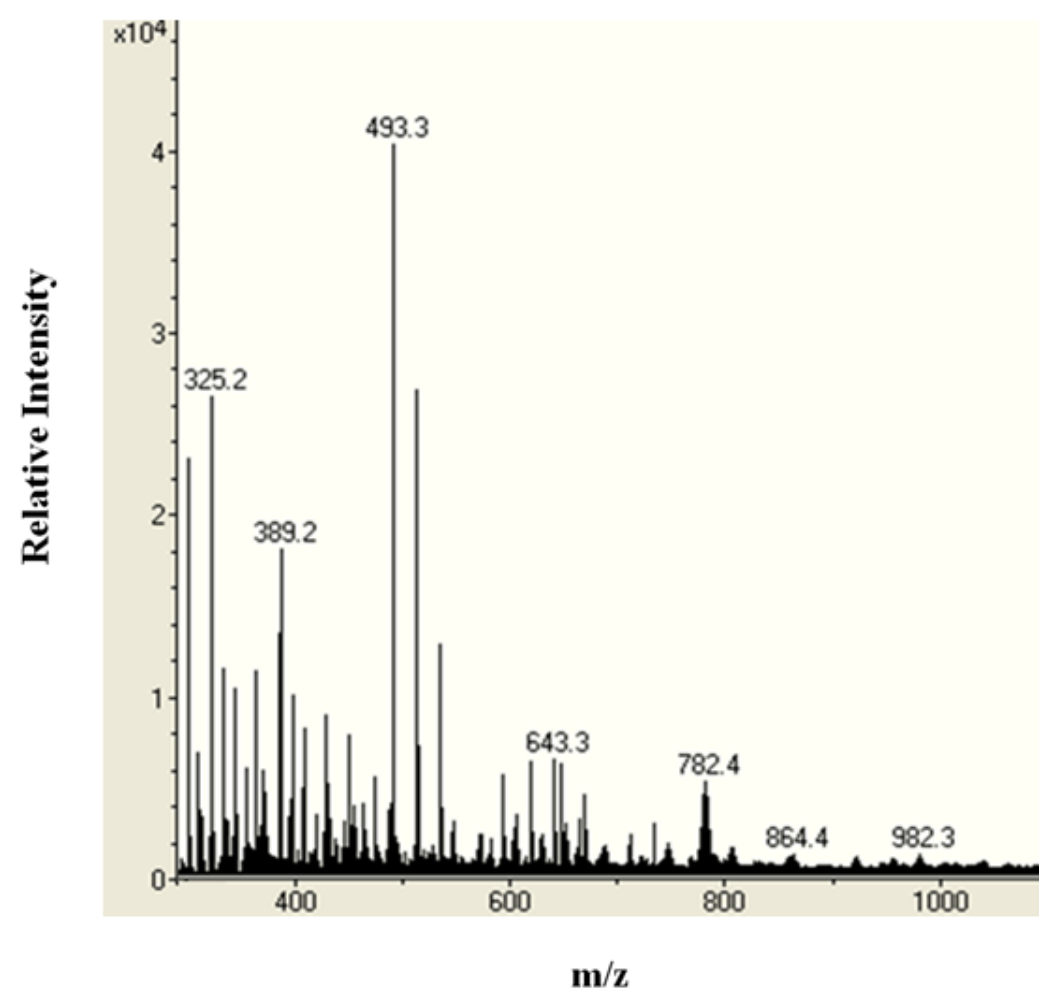

Figure 47: Mass spectrum of $\mathrm{C}(\mathrm{KAFKRK})_{3}$ in PBS after enzymatic degradation. 
Tryspin digestion was then carried out on the peptide capped GNP composites with the following peptide ratios: $100 \% \quad \mathrm{C}(\mathrm{KAFKRK})_{3}$ and $50 \% \quad \mathrm{C}(\mathrm{KAFKRK})_{3}: 50 \%$ CKAFKRK. The trypsin/versene solution was added to the GNP solutions of the same concentrations, mixed, incubated at $37^{\circ} \mathrm{C}$ and analysed after 24 hours. Refer to Appendix 3, Spectrum 29 to Spectrum 32, page 156 for all spectra.

The Au-50\% C(KAFKRK) $3: 50 \%$ CKAFKRK solution underwent a noticeable colour change from the initial pink to a colourless solution with the presence of a precipitate. This observation can be explained by a decrease in colloidal stability of the GNPs upon cleavage of the long peptide, resulting in aggregation of the GNPs. The Au- $100 \% \mathrm{C}(\mathrm{KAFKRK})_{3}$ solution did not change colour and remained pink. This indicated that the $\mathrm{C}(\mathrm{KAFKRK})_{3}$ peptide was not cleaved by the protease as the capped GNPs were still in solution.

To remove the precipitate, the solutions were centrifuged and the supernatant analysed via LC-MS connected to an ESI Q-TOF. The mass spectra of the peptides attached to the GNPs were recorded before and after cleavage. The spectrum of the $100 \% \mathrm{C}(\mathrm{KAFKRK})_{3}$ did not appear to change after the experiment, indicating that cleavage did not occur. The MS of the $50 \% \mathrm{C}(\mathrm{KAFKRK})_{3}: 50 \%$ CKAFKRK showed significant cleavage of the peptide, with $493 \mathrm{~m} / \mathrm{z}$ being the most prominent fragment peak. These results indicated that the ratio of 50:50 [CKAFKRKCoum:C(KAFKRK) ${ }_{3}$ ] was more effective in allowing for peptide cleavage.

\subsubsection{Selection of the final GNP composite}

Taking into consideration the findings of section 5.6.2 (page 76): The percentage of CKAFKRKCoum should be 10\%) and section 5.6.3., page 77: The ratio of 50:50 CKAFKRKCoum:C(KAFKRK) 3 was more effective in allowing for peptide cleavage, it was evident that a spacer group (40\%) is required to facilitate trypsin digestion.

The CME ligand was attached to GNPs as a spacer group along with $\mathrm{C}(\mathrm{KAFKRK})_{3}$ and CKAFKRKCoum in a ratio of 40:50:10 respectively. This sequence unfortunately produced extremely aggregated GNPs that were completely insoluble. It was already established that 50\% $\mathrm{C}(\mathrm{KAFKRK})_{3}: 50 \%$ CKAFKRK was water soluble, thus the next spacer decided upon was CKAFKRK to ensure water solubility of the GNP composite. The ratio attempted was $40 \%$ CKAFKRK:50\% C(KAFKRK) $)_{3}: 10 \%$ CKAFKRKCoum. Digestion was performed and proved successful on the Au-40\% CKAFKRK:50\% C(KAFKRK) $3: 10 \%$ CKAFKRKCoum system. 


\subsubsection{Evaluating the effect of peptide ratio on protease digestion}

A simple but logical enquiry became the subject of a sideline investigation: What effect would varying the loading of the cleavable peptide $\left[\mathrm{C}(\mathrm{KAFKRK})_{3}\right]$ have on the rate of protease activity? This was further investigated by preparing four different GNP solutions as illustrated in Table 10:

Table 10: GNP solutions prepared with varying percentages of $\mathrm{C}(\mathrm{KAFKRK})_{3}$, CKAFKRKCoum and CKAFKRK and respective names.

\begin{tabular}{|cc|}
\hline Name & GNP composite \\
\hline $\mathbf{9 0} \%$ & $90 \% \mathrm{C}(\text { KAFKRK })_{3}: 10 \%$ CKAFKRKCoum \\
\hline $\mathbf{7 0 \%}$ & $70 \% \mathrm{C}(\text { KAFKRK })_{3}: 10 \%$ CKAFKRKCoum: $20 \%$ CKAFKRK \\
\hline $\mathbf{5 0 \%}$ & $50 \%$ C (KAFKRK $)_{3}: 10 \%$ CKAFKRKCoum: $40 \%$ CKAFKRK \\
\hline $\mathbf{3 0} \%$ & $30 \%$ C (KAFKRK $)_{3}: 10 \%$ CKAFKRKCoum: $60 \%$ CKAFKRK \\
\hline
\end{tabular}

Each composite was subjected to protease digestion to evaluate the differences the extent of loading had on the digestion of the cleavable peptide.

Digestion was conducted on a range of concentrations of the $\mathrm{C}(\mathrm{KAFKRK})_{3}$ peptide for a period of 24 hours, after which the fragment at $493 \mathrm{~m} / \mathrm{z}$ was analysed by LC-MS connected to an ESI Q-TOF to ascertain the area of the fragment for each concentration. The areas were then plotted as a function of KAFK concentration and the calibration curve is shown in Figure 48. 


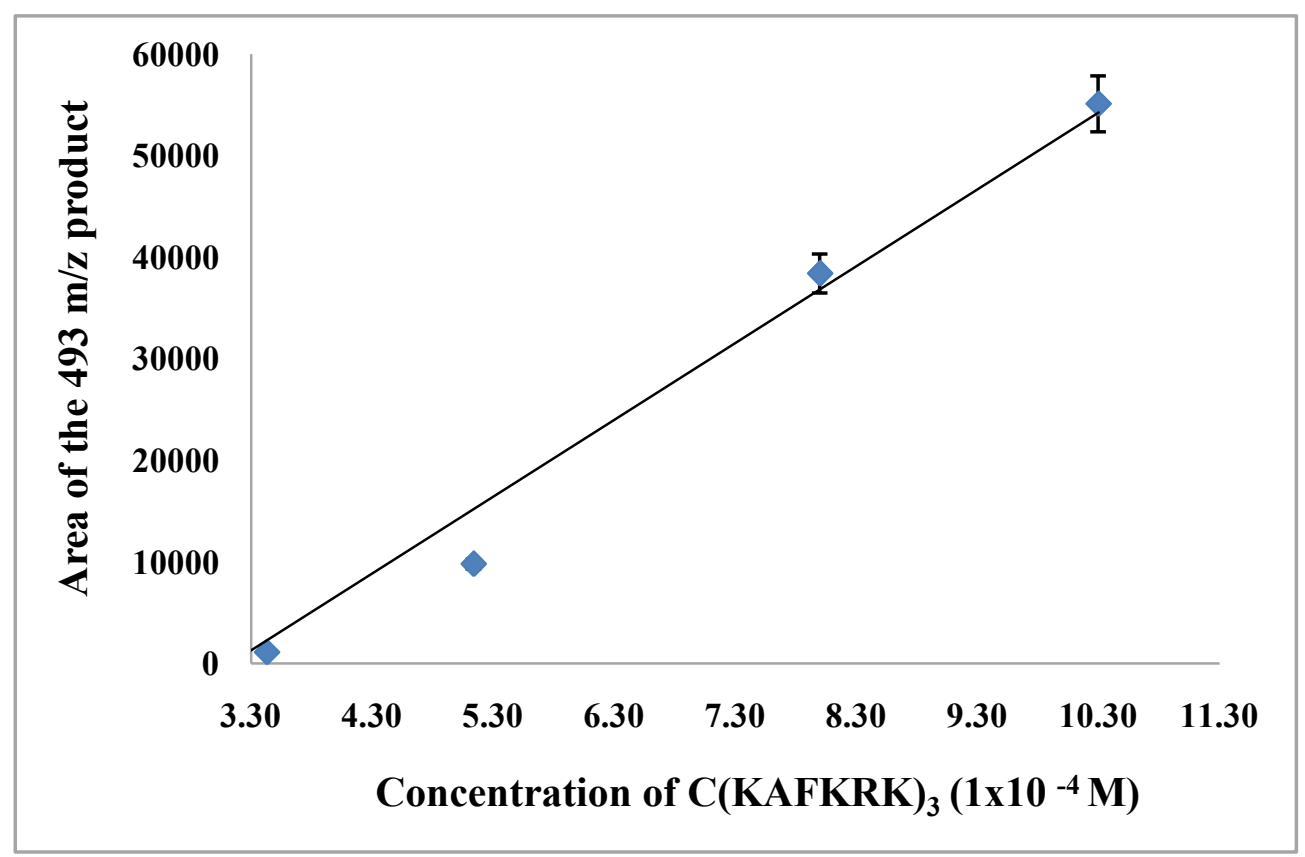

Figure 48: Calibration curve of $\mathrm{C}(\mathrm{KAFKRK})_{3}$ peptide indicating the relationship between the concentration and the area of the peptide. Each data point represents a total of three assays.

Trypsin/versene solution of the same concentrations were added to each GNP solution (90-30\%) of the same concentrations, vortexed, incubated at $37^{\circ} \mathrm{C}$ and analysed after $3,6,9$ and 24 hours. The solutions underwent a noticeable colour change from the initial bright red to a colourless solution with the presence of a precipitate. This theory concurs with the experimental observations: The $30 \%$ GNP solution changed colour at a faster rate than the $90 \%$ GNP solution [the $90 \%$ consists of more $\mathrm{C}(\mathrm{KAFKRK})_{3}$ peptide and thus remained stable for longer than the $30 \%$ covered GNPs]. To remove the precipitate, the solutions were centrifuged and the supernatant was analysed with LCMS connected to an ESI Q-TOF. The concentration of the $493 \mathrm{~m} / \mathrm{z}$ mass fragment at three hour intervals was determined using the equation generated by the calibration curve (Figure 48) of $y=$ $8 \times 10^{7} x-28917$. The results obtained are shown in Table 11 and plotted in a bar graph format for purposes of comparison (Figure 49). 
Table 11: The concentration of the mass fragment $493 \mathrm{~m} / \mathrm{z}$ obtained at three hour intervals for different GNP solutions.

\begin{tabular}{|c|c|c|c|}
\hline GNP ratio & Time (hours) & Mean Area & Concentration (M) \\
\hline \multirow{5}{*}{$90 \%$} & 3 & 9001 & $4.74 \times 10^{-4}$ \\
\hline & 6 & 97683 & $1.58 \times 10^{-3}$ \\
\hline & & & \\
\hline & 9 & 2728 & $3.96 \times 10^{-4}$ \\
\hline & 24 & 3263 & $4.02 \times 10^{-4}$ \\
\hline \multirow{5}{*}{$70 \%$} & 3 & 7850 & $4.60 \times 10^{-4}$ \\
\hline & 6 & 8236 & $4.64 \times 10^{-4}$ \\
\hline & & & \\
\hline & 9 & 4910 & $4.23 \times 10^{-4}$ \\
\hline & 24 & 1762 & $3.83 \times 10^{-4}$ \\
\hline \multirow{4}{*}{$50 \%$} & 3 & - & - \\
\hline & 6 & 10244 & $4.90 \times 10^{-4}$ \\
\hline & 9 & 9950 & $4.86 \times 10^{-4}$ \\
\hline & 24 & 9079 & $4.75 \times 10^{-4}$ \\
\hline \multirow{5}{*}{$30 \%$} & 3 & - & - \\
\hline & 6 & - & - \\
\hline & & & \\
\hline & 9 & 4837 & $4.22 \times 10^{-4}$ \\
\hline & 24 & 9230 & $4.77 \times 10^{-4}$ \\
\hline
\end{tabular}




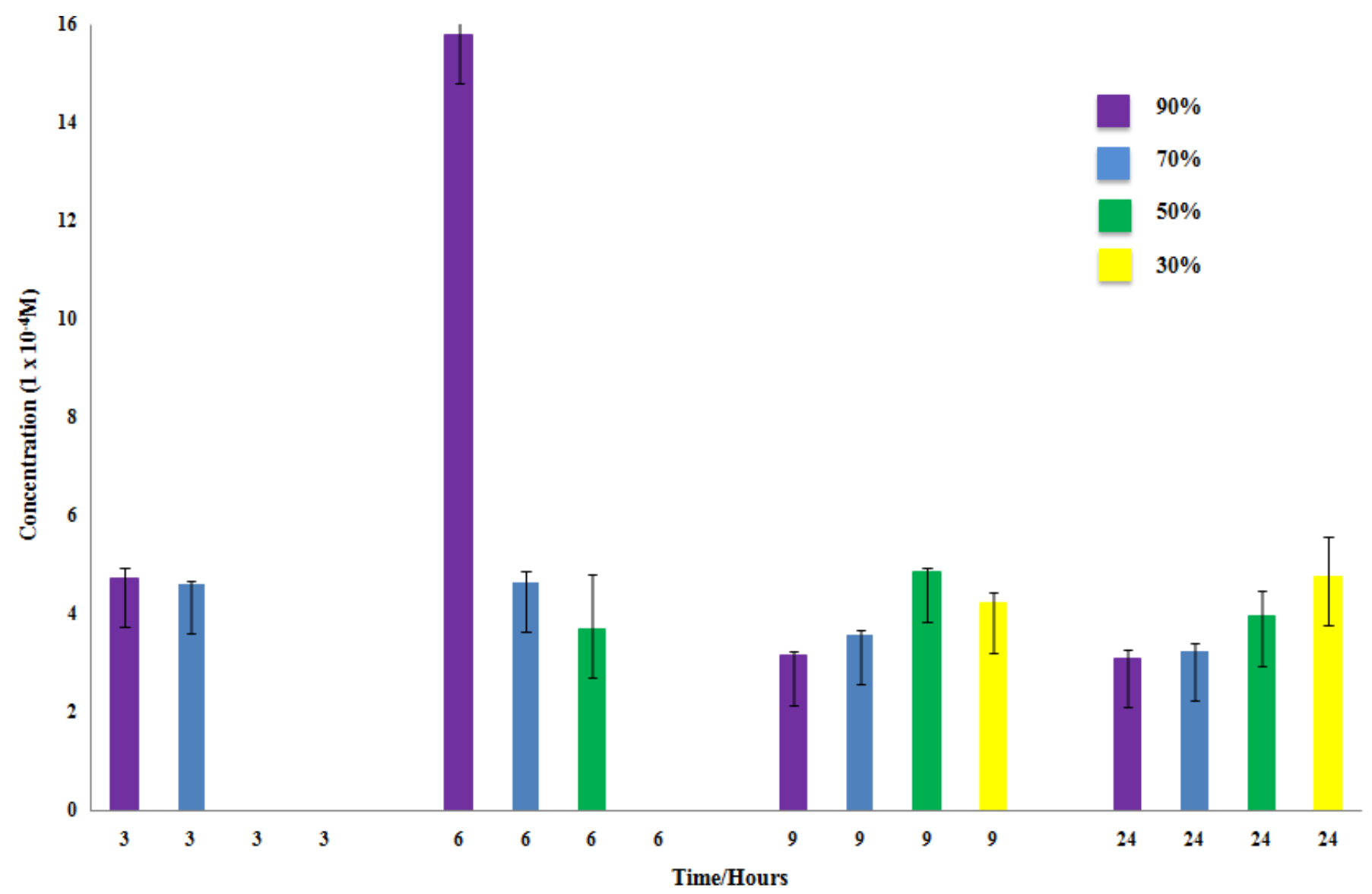

Figure 49: Bar graph illustrating the comparison of the concentrations obtained for the peak at 493 $\mathrm{m} / \mathrm{z}$ after 3-24 hours of exposure to trypsin. The $493 \mathrm{~m} / \mathrm{z}$ peak correlates to the peptide fragment resulting from the cleavage of the peptide $\mathrm{C}(\mathrm{KAFKRK})_{3}$ and can be ascribed to the fragment KAFK. The area of the $493 \mathrm{~m} / \mathrm{z}$ peak was measured for each GNP solution at each time interval. The area was converted to concentration by using the calibration graph in Figure 48 . The concentrations for each GNP solution were plotted as a function of time.

The rate of cleavage appears to be directly proportional to the extent of aggregation. The cleavable peptide $\mathrm{C}(\mathrm{KAFKRK})_{3}$ attached to the GNPs with a loading of $90 \%$ was cleaved significantly after six hours with a concentration of $15.8 \times 10^{-4} \mathrm{M}$. This can be attributed to the larger surface area of the exposed target peptide due to significantly greater dispersion of the NPs (Figure 50). The decrease in the amount of the initial cleavage product corresponded to an increase in another peak $(389 \mathrm{~m} / \mathrm{z})$ corresponding to the peptide fragment AFK. The aggregation in the lower percentages of $\mathrm{C}(\mathrm{KAFKRK})_{3}$, such as the $30 \%$ and $50 \%$, appears to delay and hinder the protease from cleaving the peptide. In the case of the higher percentages of the cleavable peptide $(70 \%$ and $90 \%)$ the product is detectable from as early as three hours, but was only detectable at six and nine hours for the $50 \%$ and $30 \%$, respectively. 

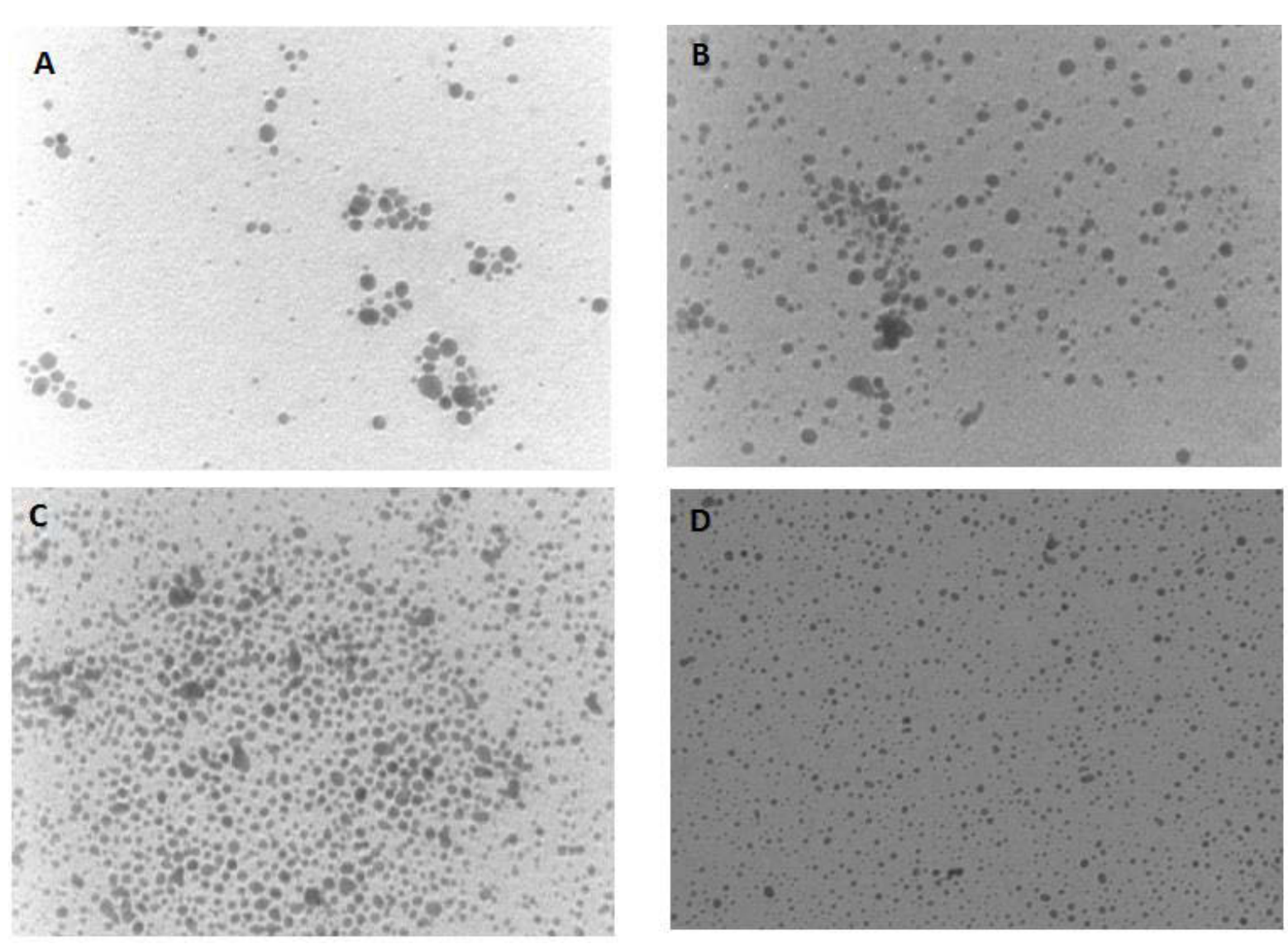

Figure 50: TEM images of GNPs in distilled water. A) 30\%, B) 50\%, C) 70\%, D) $90 \%$.

The results shown in sections 5.6.3. (page 77) and 5.6.4. (page 80) differ. In the former, it was observed that the peptide in a $100 \% \mathrm{C}(\mathrm{KAFKRK})_{3}$ functionalised GNP solution did not cleave on exposure to trypsin for 24 hours. In the latter, however, it is evident that the peptide on a $90 \%$ $\mathrm{C}(\mathrm{KAFKRK})_{3}$ functionalised GNP solution did cleave, which indicates that a spacer is essential as a $100 \% \mathrm{C}(\mathrm{KAFKRK})_{3}$ functionalised GNP solution is perhaps too sterically crowded.

\subsubsection{Effect of peptide encapsulation on fluorescence intensity of Coumarin}

Emission spectroscopy was performed before and after a 24 hour digestion period to ascertain the effect of the longer peptides on the fluorescence intensity and on the shift of $\lambda_{\max }$. The composite Au-40\% CKAFKRK:50\% C(KAFKRK) $3: 10 \%$ CKAFKRKCoum was digested with trypsin and fluorescence was measured before and after cleavage. It can be seen from Figure 51 that the emission was enhanced after cleavage, indicating that the long peptide in fact "shielded" the fluorescence intensity of the fluorophore to some extent. In addition to an increase in intensity, a red shift of the $\lambda_{\max }$ after cleavage was also observed.

These two observations could be explained either by: 
- A change in the local environment of the fluorophore:

$\circ$ prior to degradation, the fluorophore is surrounded by the „longe peptide $\mathrm{C}(\mathrm{KAFKRK})_{3}$ and is not affected by the surrounding water molecules. After cleavage of the „longe peptide, the water molecules become more accessible to the fluorophore. This change in environment causes a red shift of the fluorescence maximum of the fluorophore as well as an increase in the emission intensity. This phenomenon is expected as Coumarin molecules are known to exhibit a high degree of sensitivity to their local environment, including polarity and viscosity. ${ }^{\mathbf{1 3 0}}$ An increase in the solvent polarity results in emission at longer wavelengths, thereby validating the observed red shift of the fluorescence maximum, ${ }^{37}$

- FRET occurring between the aromatic groups of phenylalanine and Coumarin:

○ prior to cleavage of the „longee peptide, FRET occurs between the Coumarin-3carboxylic acid (donor) and the phenylalanine (acceptor) thereby diminishing the fluorescence emission intensity of the fluorophore. The cleavage of the „longe peptide (and subsequent removal of phenylalanine groups) effectively prevents the occurrence of FRET, resulting in an enhanced fluorescence emission intensity and red shift of the $\lambda_{\max }$ of the Coumarin moiety.

Further studies are necessary to confirm the mechanism that caused the increased fluorescence emission intensity and the shifting of the $\lambda_{\max }$.

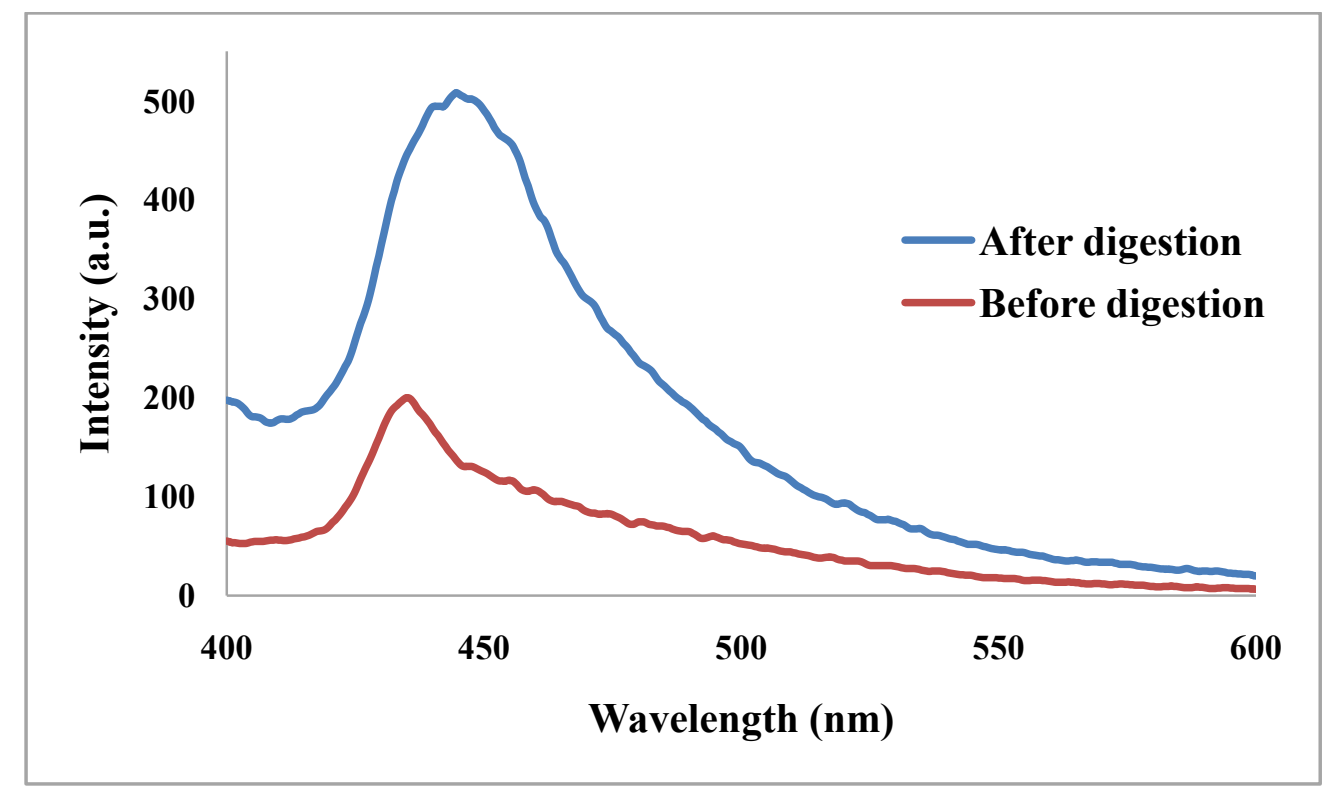

Figure 51: Fluorescence spectra illustrating the difference in emission intensity of peptide capped GNPs before and after cleavage with trypsin. 


\subsubsection{Transmission electron microscopy}

It is essential to know the fate of the GNPs after treatment with whole cells: Do the cells internalize the GNPs or do the GNPs remain bound to the cell membrane? TEM was used to verify the cellular uptake of GNPs in A549 and MDBK cell lines. Figure 52 and Figure 53 shows typical images of A549 cells and MDBK cells, respectively, exposed to GNPs at a concentration of $2.09 \times 10^{-11} \mathrm{M}$ for 24 hours at $37^{\circ} \mathrm{C}$. GNPs were found at the cell periphery (Figure $52 \mathrm{~A}$ ) although evidence for accumulation of large quantities of GNPs inside the cell was clearly visible by TEM (Figure 52 AC). As shown in Figure $52 \mathrm{~B}$ and Figure 53 A, GNPs appeared to be trapped in the vesicles (most likely due to endosomal entrapment) in the cytoplasm which is in agreement with results by other groups. ${ }^{131,132,43,133,134}$ The higher magnification TEM images (Figure 52 B, C Figure 53 A) show that these GNPs were aggregated in the vesicles in the cytoplasm, rather than attached to the membrane only. It is likely that some GNPs escaped from the vesicles as NP escape depends on the surface functionalities as is evident by the presence of individual GNPs in Figure $52 \mathrm{C}$ and Figure 53 B. $^{134}$ 

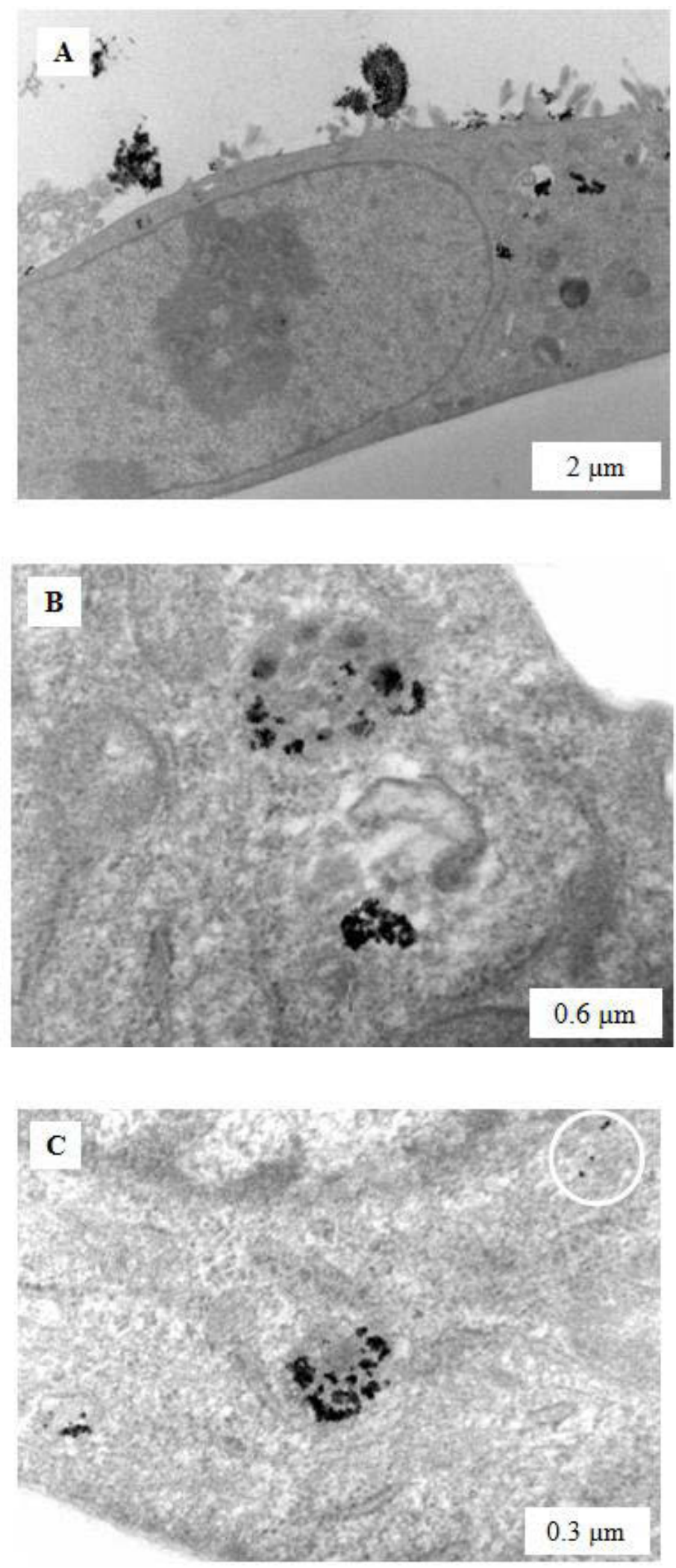

Figure 52: Cross-section TEM images of A549 cells incubated with GNPs for 24 hours. A) GNPs were seen at the cell periphery. B) Magnification of the aggregate GNPs in the cytoplasm indicating the uptake of GNPs in cellular vesicles. C) Free GNPs (white circle) indicating the possibility that some GNPs escaped from the vesicles. 

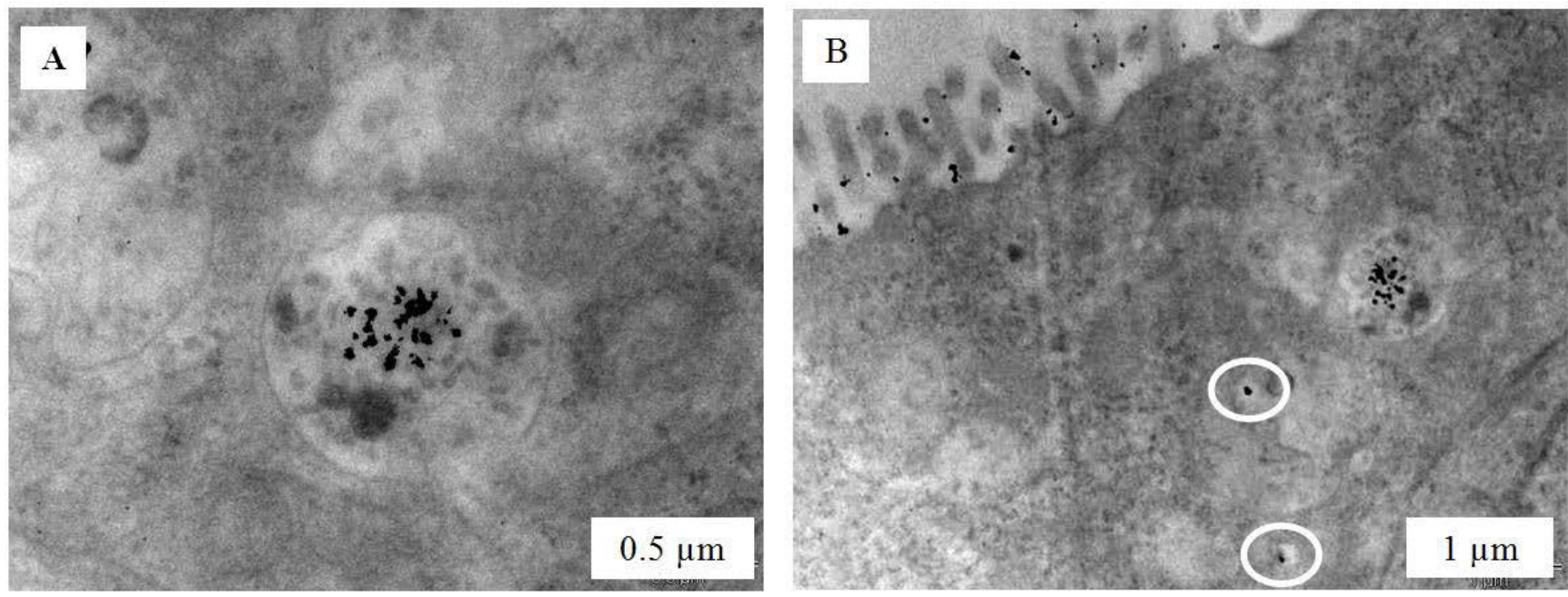

Figure 53: Cross-section TEM images of MDBK cells incubated with GNPs for 24 hours. A) Magnification of the aggregated GNPs in the cytoplasm indicating the uptake of GNPs in cellular vesicles. B) Free GNPs (white circles) indicating the possibility of GNPs escape from the vesicles.

\subsubsection{Mechanism of cellular uptake of peptide capped GNPs}

To date, several reports have discussed the internalization of GNPs into the cells by an endocytic pathway and this is generally accepted as the most likely method of transport of GNPs into cells. ${ }^{131,132}$ Endocytosis is a conserved process in eukaryotes where extracellular substances are taken up into cells usually by the invagination of plasma membrane, forming vesicles. ${ }^{\mathbf{1 3 5}}$ Generally, endocytosis occurs by multiple mechanisms that fall into two broad categories, namely ,phagocytosis ${ }^{\text {ee }}$ (the uptake of large particles), and ,pinocytosise (the uptake of fluid and solutes). ${ }^{\mathbf{1 2 8}}$ Upon binding to cell surface receptors, NP-based drug delivery systems are internalized by phagocytosis, otherwise known as receptor-mediated endocytosis. ${ }^{35,128}$ As the GNPs adhere to the cell membrane, it is stimulated to form a small pocket locally around NPs, thereby gradually enclosing the GNPs. In this process the NP complex is able to enter the cell in vesicles known as early endosomes. When the NPs are eventually internalized they are then aggregated in a closed pocket (vesicle/endosome). ${ }^{\mathbf{1 3 2}}$ Due to the evidence of aggregation of the GNPs in the cells (Figure 52 and Figure 53, pages 87 and 88) it is most likely that the GNPs are taken up by the cells through phagocytosis. $^{132}$ 


\subsubsection{Cytotoxicity studies of the peptide capped GNPs}

The purpose of this assay was simply to measure the toxic effect of the functionalised GNPs on the A549 and MDBK cell lines. Optimisation of the MTT assay was previously performed by a master's student in our group. ${ }^{\mathbf{1 3 6}}$ The optimal number of cells used in the testing as well as the optimal time required for solubilisation of the formazan crystals was previously established. The use of $6 \times 10^{4}$ cells per well produced the least standard deviation and a solubilisation of two hours had the lowest standard deviation between samples. Thus, all tests were performed by plating cells at a density of $6 \times 10^{4}$ cells per well and absorbance readings were obtained after a solubilisation of two hours.

To ensure that the testing technique was not flawed, the sensitivity of the A549 and the MDBK cells to cadmium was assessed using various concentrations of the control. Cadmium was chosen as it is a widely used control in other MTT-based assays and has been found to be cytotoxic to both cell lines. ${ }^{137}$ As seen in Figure 54, a concentration as low as $4.5 \times 10^{-3} \mathrm{M}$ cadmium was sufficient to produce a significant kill in the MDBK cell line, whereas a concentration of greater than $5.3 \times 10^{-}$

${ }^{2} \mathrm{M}$ cadmium was required to produce a significant kill of the A549 cells (Figure 55). It is evident by these results that the MDBK cell line is much more sensitive to cadmium than the A549 cells. 


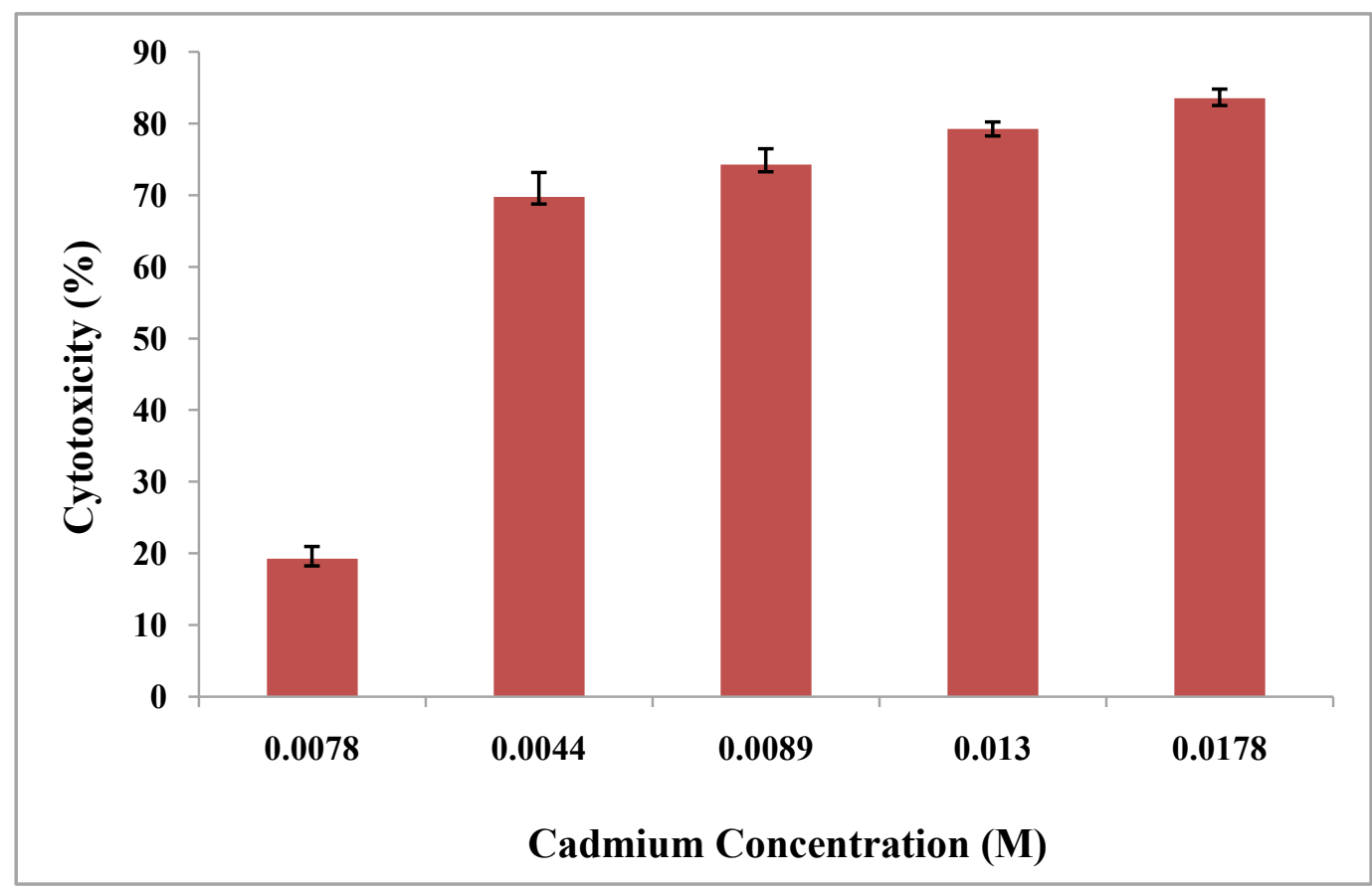

Figure 54: Graph showing the cytotoxic effect of different cadmium concentrations on MDBK cells ( $5 \times 10^{4}$ cells per well after 42 hours incubation with cadmiun). Error bars show the SD between five replicates.

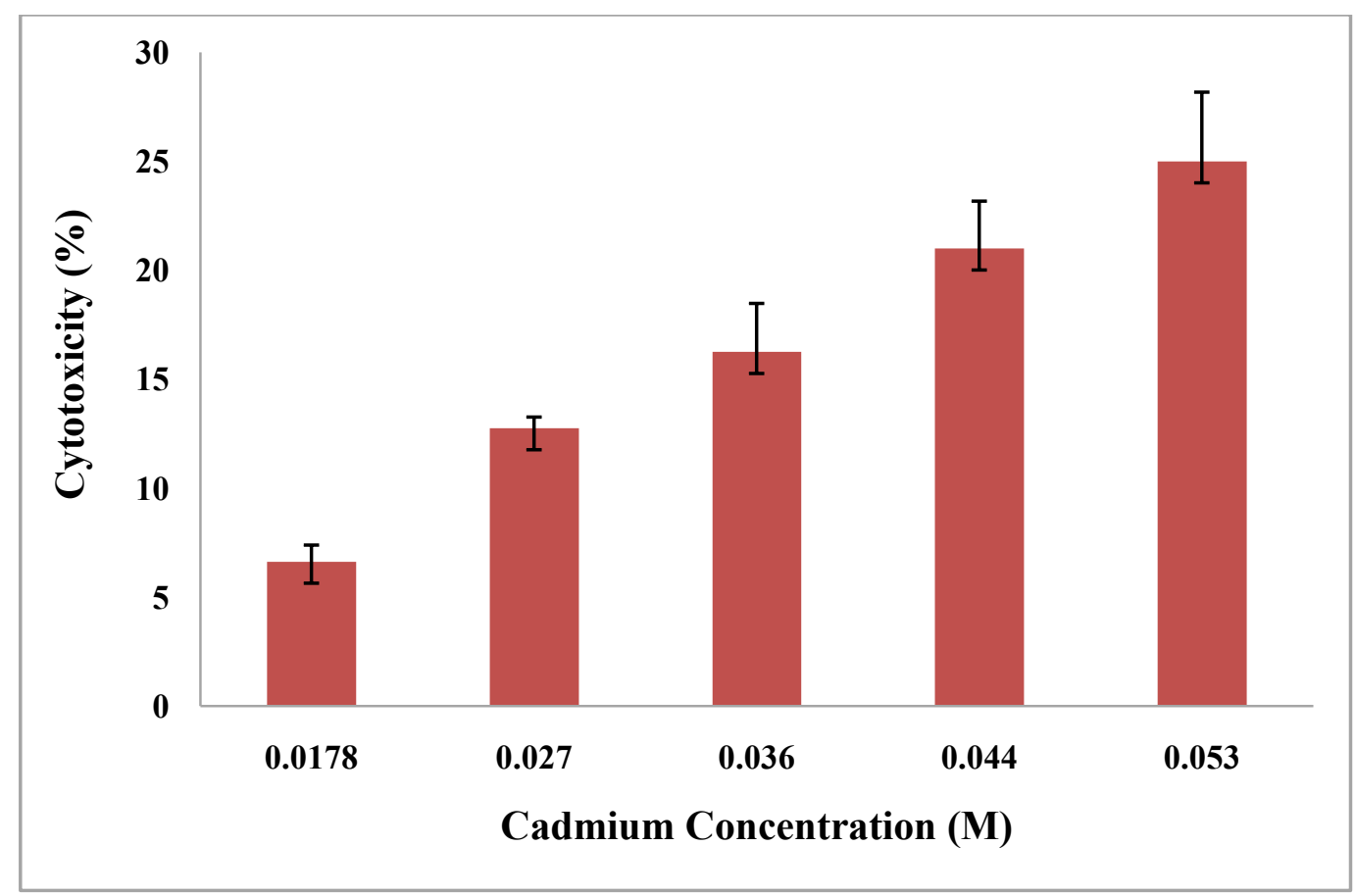

Figure 55: Graph showing the cytotoxic effect of different cadmium concentrations on A549 cells ( $5 \times 10^{4}$ cells per well after 42 hours incubation with cadmiun). Error bars show the SD between five replicates. 
The peptide capped GNPs were then analysed for cytotoxic effects using the MTT assay. Cadmium was used as the control in each assay to ensure the methodology was correct.

A range of concentrations of the peptide capped GNPs were tested to determine their toxicity to the cells. The lowest concentration used was the same concentration of peptide capped GNPs used in TEM and confocal microscopy testing $\left(2.09 \times 10^{-11} \mathrm{M}\right)$ and the highest was the concentration that did not produce significant colouration of the solution that would affect the assay $\left(9.2 \times 10^{-11} \mathrm{M}\right)$. It was found that the peptide capped GNPs were not cytotoxic to either the MDBK (Figure 56) or the A549 (Figure 57) cell line.

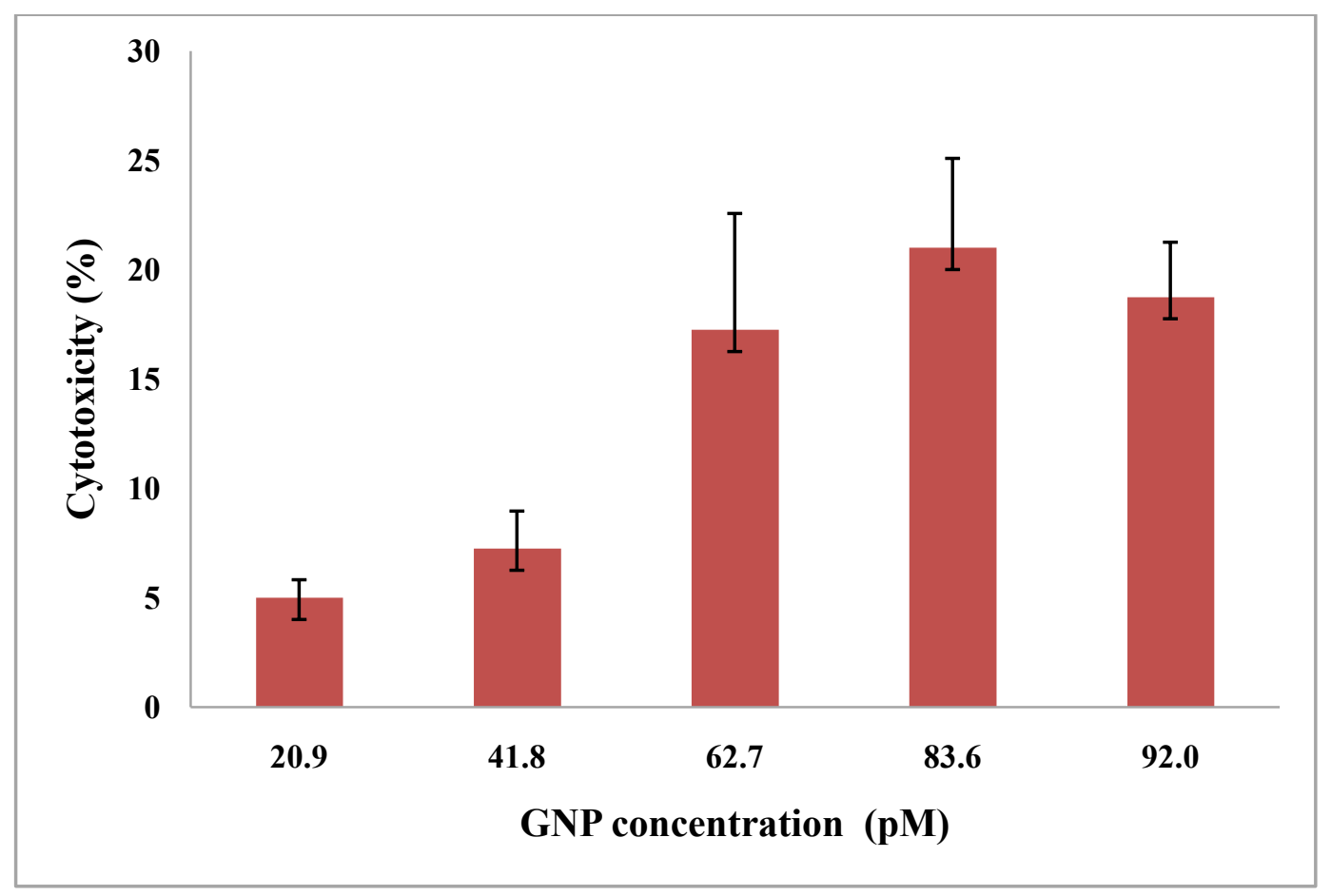

Figure 56: Graph showing cytotoxicity of various peptide capped GNP concentrations on MDBK cells ( $5 \times 10^{4}$ cells per well after 42 hours incubation with GNPs). Error bars show the SD between three replicates. 


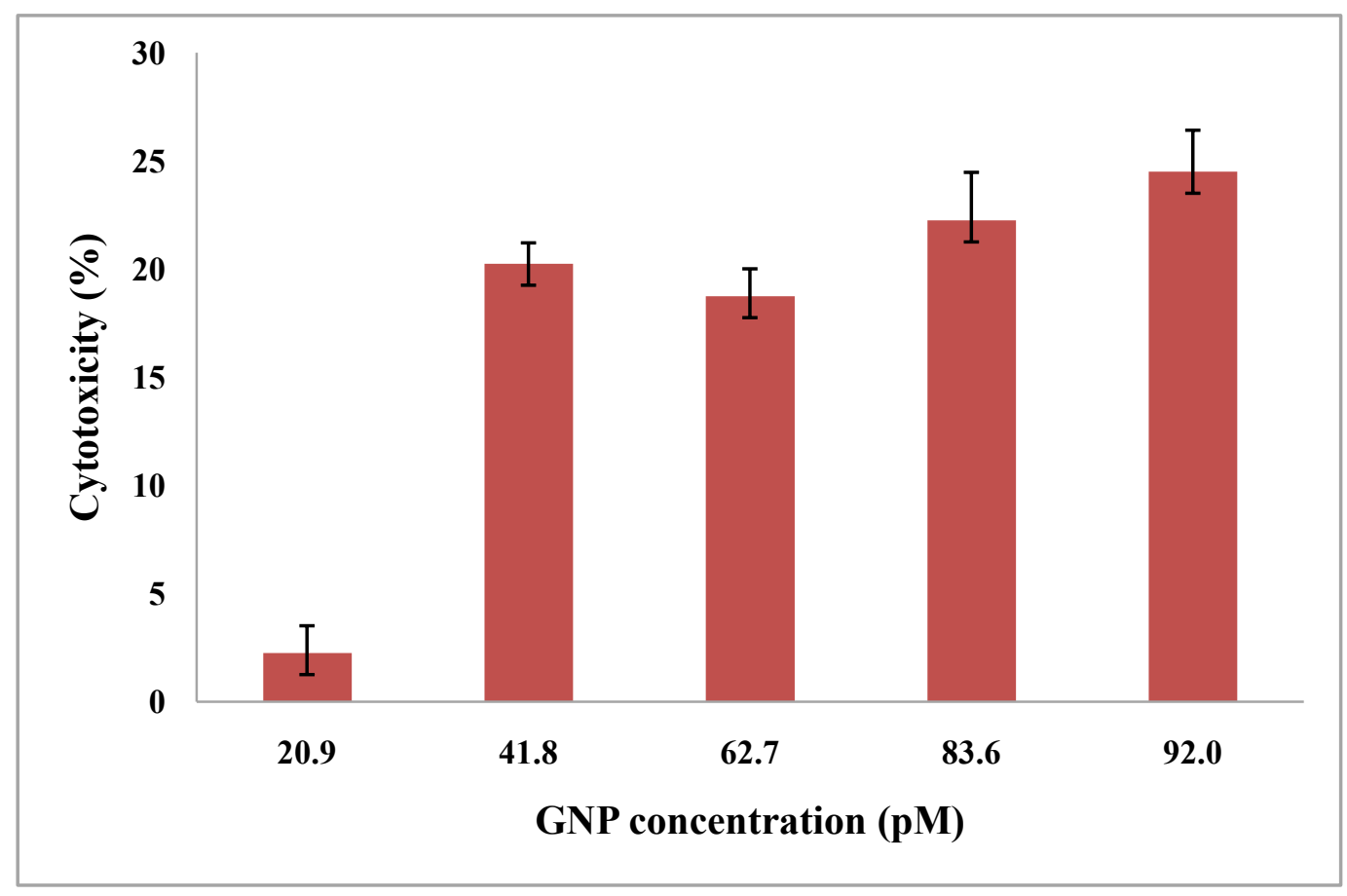

Figure 57: Graph showing cytotoxicity of various peptide capped GNP concentrations on A549 cells ( $5 \times 10^{4}$ cells per well after 42 hours incubation with GNPs). Error bars show the SD between three replicates.

\subsubsection{Confocal microscopy results of peptide capped GNPs in whole cells}

The experimental procedure followed for the confocal microscopy analyses was done as consistently as possible to ensure reproducibility. For each assay, three replicates were done (three coverslips were used). The cells were subjected to peptide capped GNP solutions of the same concentration $\left(2.09 \times 10^{-11} \mathrm{M}\right)$ for differing time periods (with exception of the controls that were not subjected to peptide capped GNPs). Approximately five images were captured per coverslip to ensure a representative image was obtained. Different areas of the coverslip were captured to prevent the occurrence of bleaching of the fluorophore. This particular experimental protocol was followed to prevent the introduction of spurious results that could be caused by excessive handling. An oil immersion was used for the objective lens to increase the resolution of the microscope. All parameters, such as pinhole and brightness, were maintained at a constant value throughout the testing (refer to section 7.6.2., page 126 for the complete experimental details).

\subsubsection{Verification of cellular uptake of peptide capped GNPs}

To validate the internalization and intracellular distribution of GNPs, MDBK and A549 cells were incubated with the GNPs for various time intervals and the intracellular fluorescence signal was 
imaged using a CFLSM. Prior to analysis the cells were washed with PBS in triplicate to ensure that only fluorescence due to uptake into cells is studied. The emission of the labelled CKAFKRKCoum GNPs produced blue fluorescence and the position and intensity represented the location. Control cells (Figure 58 A-B) and cells treated with non-functionalised GNPs alone (Figure $58 \mathrm{C}$-D) displayed faint fluorescence. In contrast, bright fluorescence was observed throughout the cells incubated with Au-40\% CKAFKRK:50\% C(KAFKRK) $3: 10 \%$ CKAFKRKCoum, clearly indicating that a substantial amount of functionalised GNPs had entered the cells, with most of the internalized GNPs localizing in the cytoplasm compartment. The complete internalization of GNPs, as opposed to simple surface attachment, was validated using the optical slicing function (Z-stack) of the confocal microscope to produce comprehensive 3-D images of the cells. Optical-section images at different depths were obtained by changing the focal plane of the confocal microscope. Compound images of the fluorescence of the MDBK and A549 cells at different depths are shown in Figure 60 and Figure 61 respectively. These optical-section images indicate that the GNPs were uniformly distributed within the cytoplasm.

\subsubsection{Time taken for complete cellular uptake of peptide capped GNPs and verification of mechanism of internalization}

Figure 58 E-F illustrates the cell lines MDBK and A549, respectively, after treatment with functionalised GNPs for one minute. As previously reported, internalization of GNPs by endocytosis occurs very rapidly (usually within a few minutes) as clearly demonstrated by the rapid uptake of the GNPs in a mere minute. This further confirms the mechanism of internalization being endocytosis. $^{45}$

\subsubsection{Proof of experimental concept}

Figure 59 G-H present CFLSM images of the cell lines MDBK and A549 after treatment with functionalised GNPs for three hours. The fluorescence intensity of the MDBK cell line appeared to decrease whereas the intensity of the A549 remained the same. This phenomena also occurred at four (Figure $59 \mathrm{I}-\mathrm{J}$ ) and 24 hours (Figure $59 \mathrm{~K}-\mathrm{L}$ ).

The initial hypothesis was that the tumour cell line (A549) would cleave the peptide attached to the GNPs at an accelerated rate (due to the over-proliferation of proteases), thereby exposing the fluorophore, resulting in an enhanced fluorescence intensity. The healthy cell line (MDBK) on the other hand was expected to cleave the peptide at a considerably slower rate than the tumour cell line (healthy cell lines have considerably less proteases than cancer cell lines). As a result the MDBK 
cell line would be much slower in exposing enhanced emission, hence resulting in a fairly constant level of fluorescence.

According to the experimental observations the fluorescence intensity of the cancer cell line A549 appeared constant and the healthy MDBK cell line appeared to gradually decrease with increasing exposure time to the GNPs. This can possibly be explained by the aggregation observed in the TEM images of the GNPs in the MDBK and A549 cell lines (Figure 52 and Figure 53).

It has been reported that aggregation of GNPs quenches the emission intensity of a fluorophore (most likely due to the effect of shielding of the fluorophore by surrounding peptide capped GNPs). This phenomenon was utilised by Huang et al. $(2007)^{\mathbf{8 1}}$ for the sensing of $\mathrm{Hg}^{\mathrm{II}}$ ions based on fluorescence quenching through $\mathrm{Hg}^{\mathrm{II}}$-induced aggregation of GNPs. Kim et al. (2009) ${ }^{\mathbf{1 3 8}}$ utilised the fluorescence quenching effect that $\mathrm{CdS}$ quantum dots exhibit upon aggregation to develop a fluorescence-based detection of point mutation in DNA sequences and Chen et al. (2009) ${ }^{\mathbf{1 3 9}}$ developed a new fluorescence sensor for the detection of $\mathrm{Cu}^{2+}$ ions based on the aggregation induced fluorescence quenching of glutathione capped GNPs. It is quite evident by examination of the TEM images (Figure 52 and Figure 53) that the aggregation of the GNPs in both cell lines occurred to the same extent, thus the fluorescence intensity of the GNPs in both cell lines should be diminished to the same extent. The TEM results, however, were obtained after exposure to the cells for 24 hours. As was seen in the trypsin digestion experiment, considerable cleavage of the peptides occurs by the enzymatic process. The protease enzymes in the two types of living cells cannot be directly compared with the trypsin studies, but it is possible that the aggregation in the cancer cells could be the result of reduced solubility of the GNPs due to cleavage of the long peptide chains. It is expected that the fluorescence of the peptide capped GNPs in the A549 cell should increase, but due to the apparent aggregation over time, the fluorescence intensity appeared to remain constant. The same can be said for the MDBK cell line: It is expected that the fluorescence intensity of the peptide capped GNPs in the MDBK cell line should remain constant but due to the aggregation of the GNPs, the emission intensity of the fluorophore appeared to decrease with time.

The correct mechanism causing the change in fluorescence emission intensity is yet to be elucidated. As previously hypothesised in section 5.6.6., page 84, the change could likely be due to either a change in the local environment of the fluorophore or the occurrence of FRET between the aromatic groups present in the peptide and the Coumarin. Further research is required to confirm either argument. 


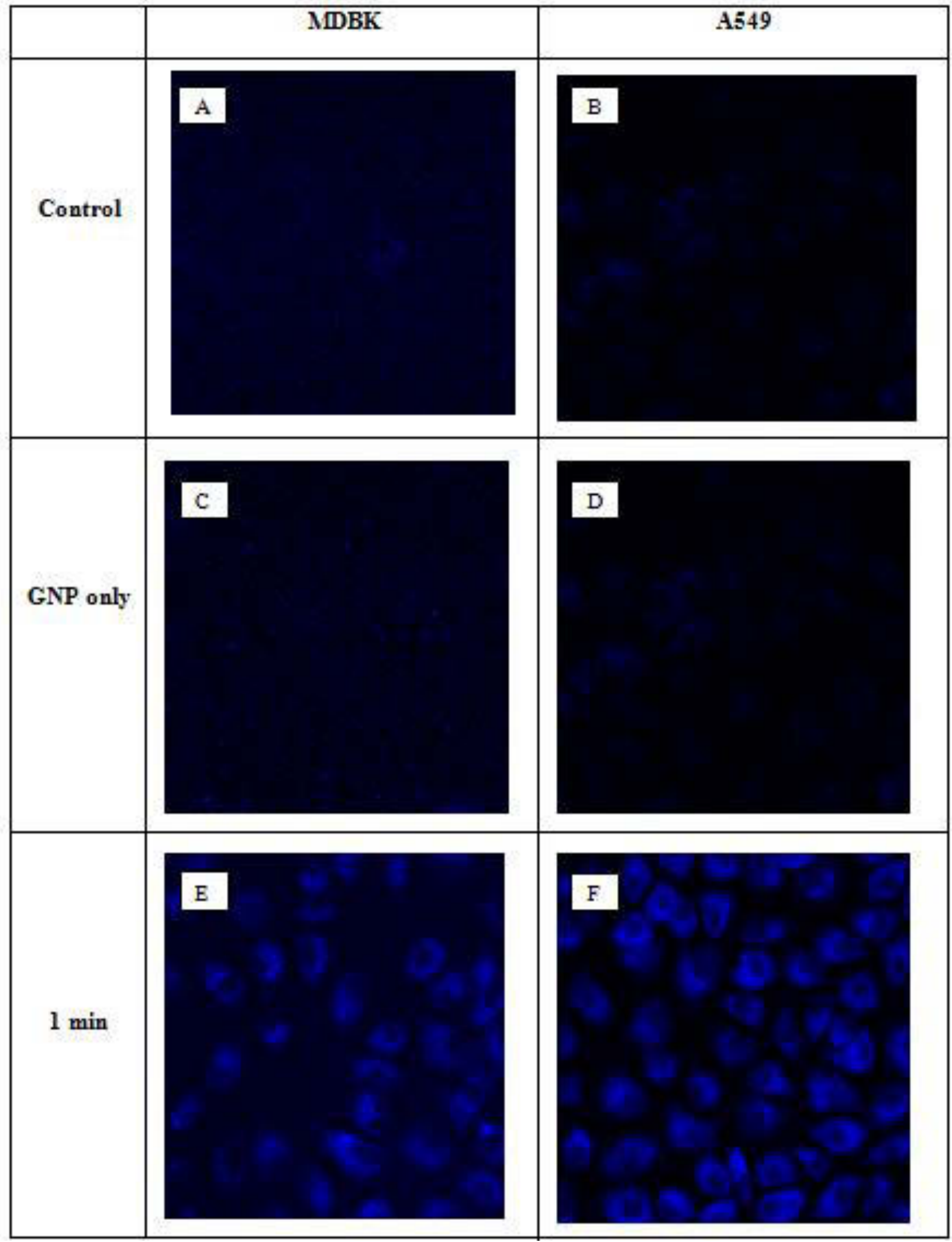

Figure 58: Confocal microscope images of MDBK (left) and A549 (right) cells. A-B) Control cells. C-D) Cells treated with non-functionalised GNPs. E-F) Cells treated with peptide functionalised GNPs for 1 minute. 


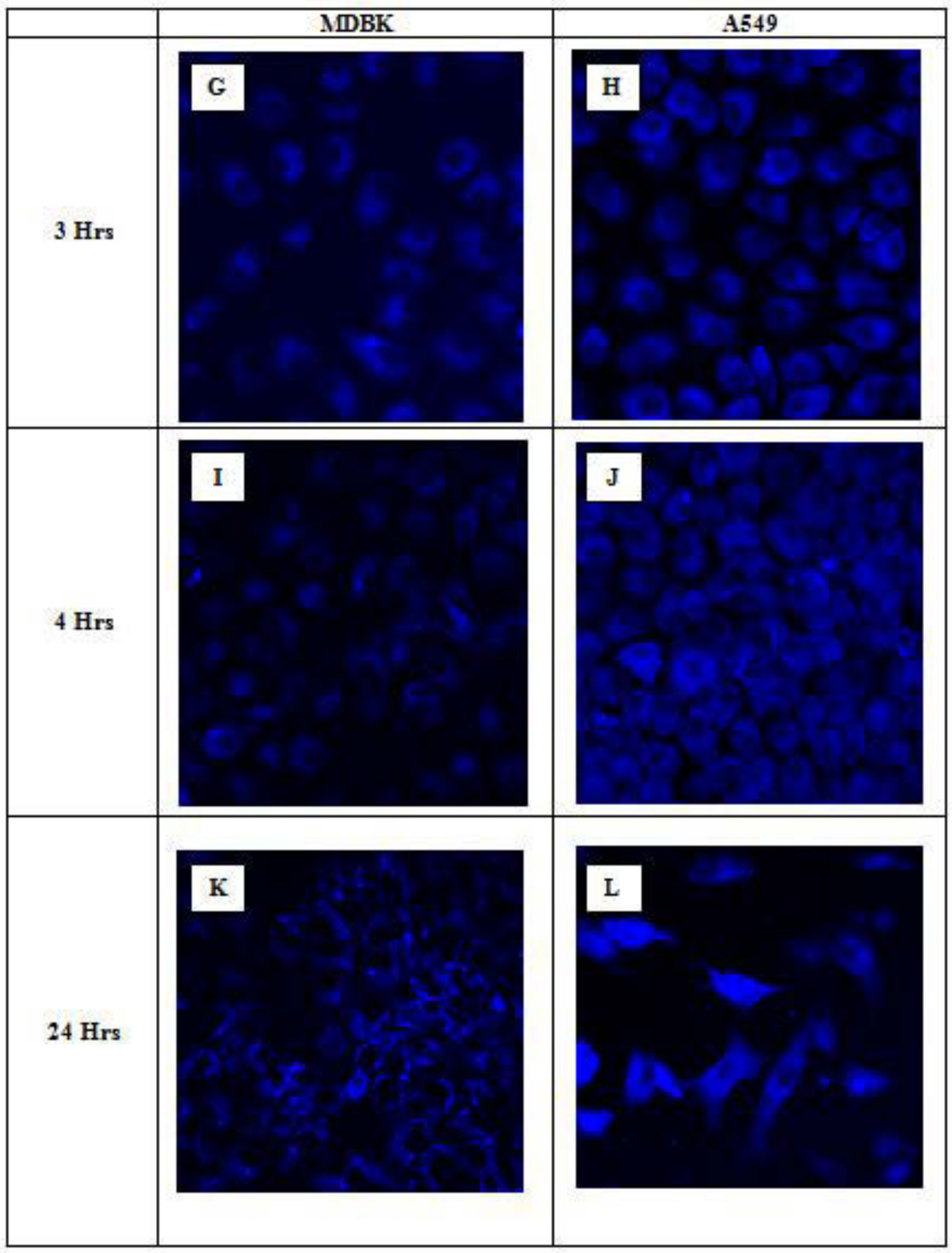

Figure 59: G-H) Cells treated with peptide functionalised GNPs for three hours. I-J) Cells treated with peptide functionalised GNPs for four hours. K-L) Cells treated with peptide functionalised GNPs for 24 hours. 


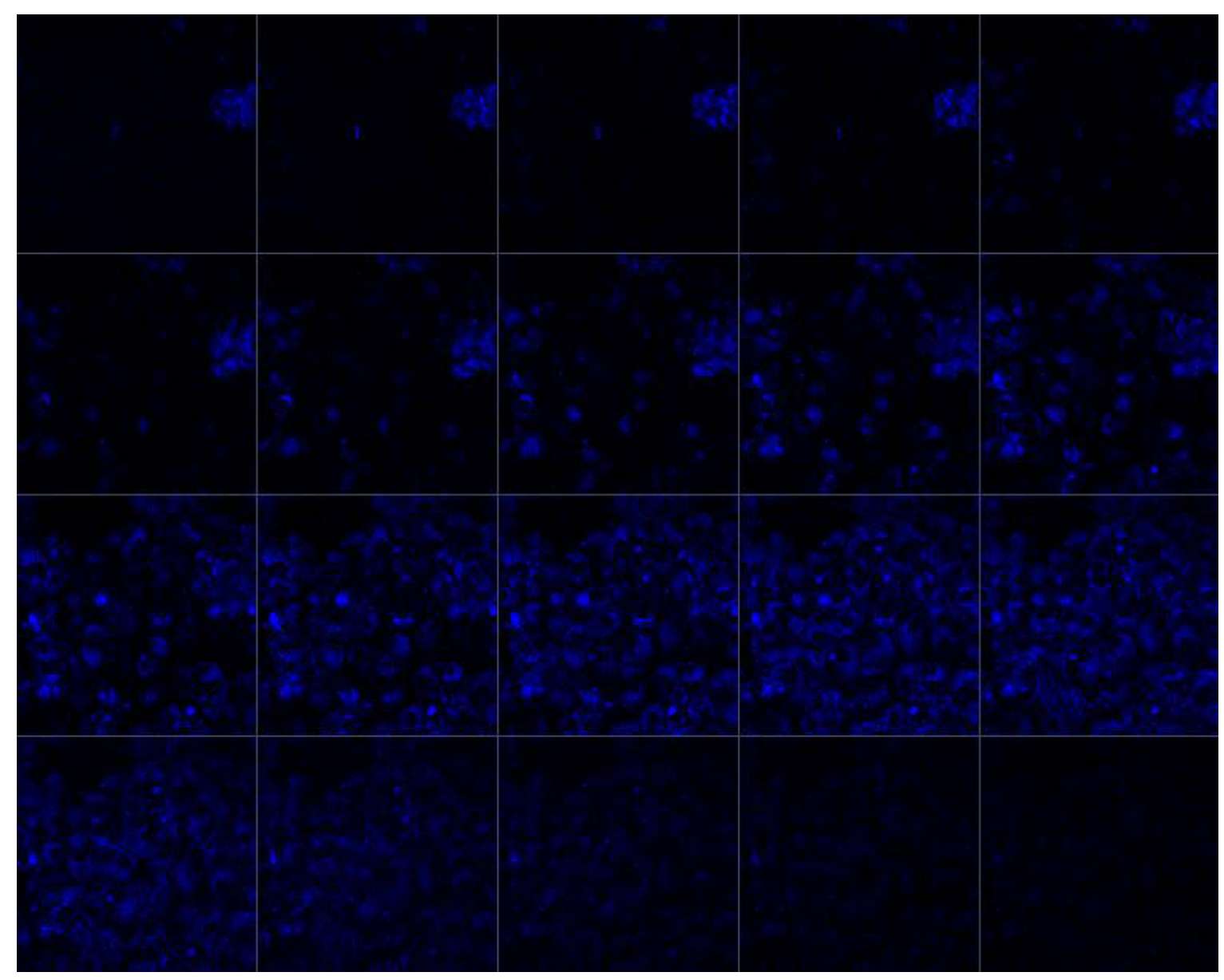

Figure 60: Optical-section images of the peptide cappped GNPs in MDBK cell line after 24 hours.

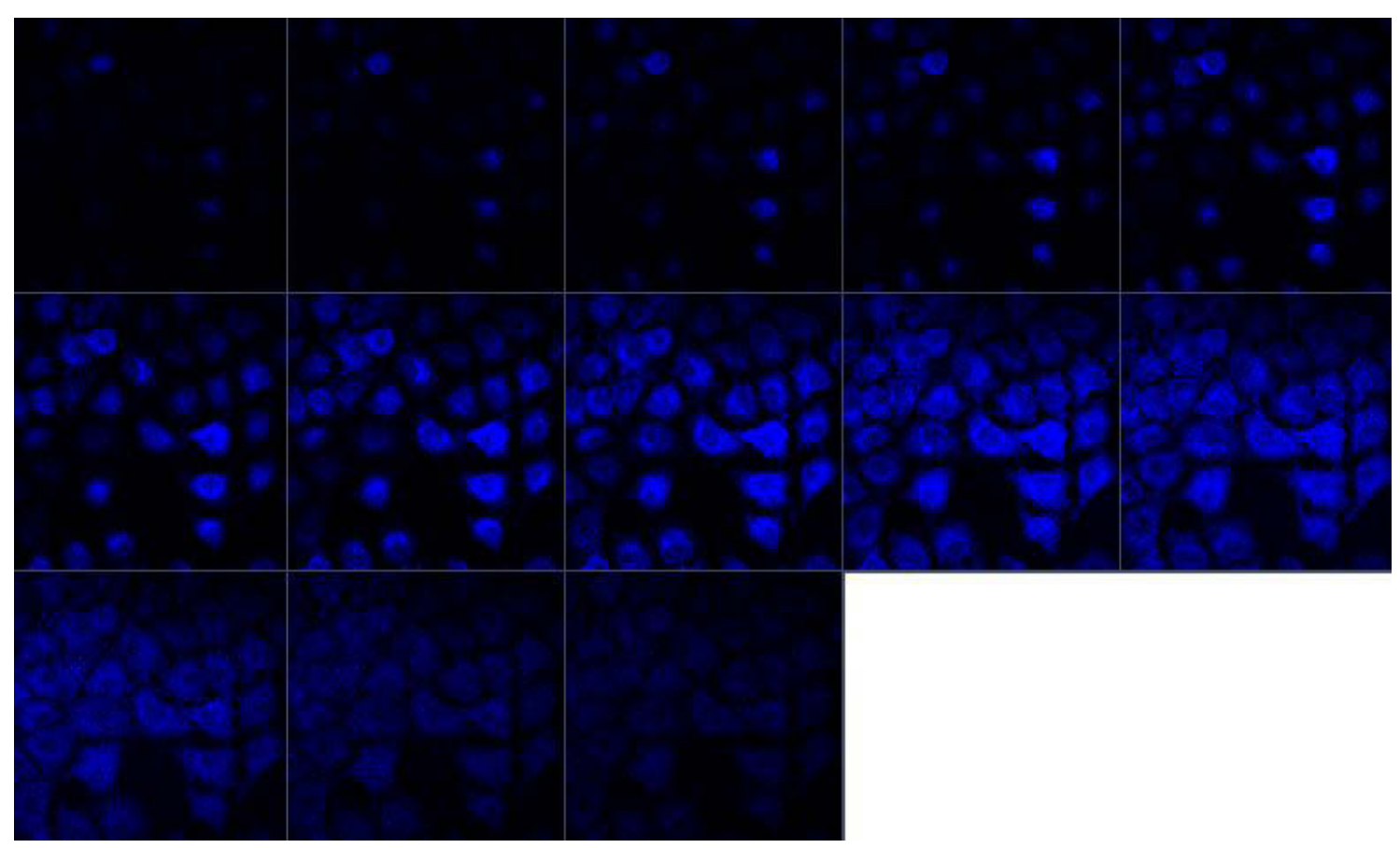

Figure 61: Optical-section images of the peptide cappped GNPs in A549 cell line after 24 hours. 


\section{CHAPTER 6: CONCLUSION}

This project presented a novel prodrug system that was activated by the proteases expressed in the region of cancer cells and remained intact in the region of the healthy cells. This was demonstrated by the functionalisation of GNPs with two peptides of different lengths, the shorter peptide consisting of a terminal fluorophore. Exposure of a cancer cell line to the peptide capped GNPs represented an increased rate of peptide cleavage due to the over-expression of proteases present in the cells, which consecutively lead to an enhanced level of fluorescence emission. In the healthy cells however, the rate of cleavage was insignificant due to the lower levels of proteases, which consequently resulted in considerably lower levels of fluorescence.

This system has the potential to improve the efficacy of current chemotherapy drugs by selective tumour cell activation and facilitate the ability to visualize the difference between tumour and normal cells. This design concept could be useful for therapeutic and diagnostic studies. 


\section{CHAPTER 7： EXPERIMENTAL}

\subsection{PEPTIDE SYNTHESIS}

All reagents and solvents were purchased commercially and, unless otherwise stated used without further purification. Rink amide resin $\left(0.61 \mathrm{mmol} . \mathrm{g}^{-1}\right)$ and protected amino acids were purchased from Iris Biotech GmbH (Marktredwitz, Germany) and GL Biochem (Shangai) Ltd. Solvents for peptide synthesis were from Sigma-Aldrich and Merck. Coumarin-3-carboxylic acid was from Aldrich.

Manual SPPS was performed using a fritted-filter sintered glass reaction vessel attached to a vacuum pump to remove excess solvents and a nitrogen line to agitate the solution and mix the reagents. The absorbance of $20 \%$ piperidine was determined using a Perkin Elmer 35 Lambda UVvis spectrophotometer. The peptides were also synthesised by SPPS on a CEM Liberty automated peptide synthesiser using Fmoc chemistry. A Stuart Scientific Orbital shaker 501 was used during the cleavage of the peptide from the resin. A Vacuubrand GmbH high vacuum pump was used to remove cleavage mixtures. The final pure products were lyophilised using a VirTis BenchtopK freeze dryer.

Reversed-phase liquid chromatography was carried out using a Younglin Acme high pressure liquid chromatograph (HPLC). Preparative HPLC was done using a C18 column $(150 \times 21.2 \mathrm{~mm})$ with acetonitrile (AcN) - water (both containing 0.1\% TFA) as mobile phase. The wavelength was set at $215 \mathrm{~nm}$. Relatively pure peptides were purified using Supelco Discovery DSC-18 solid phase extraction (SPE) syringes.

LC-MS was performed on all crude and purified peptides to confirm the purity and mass of the synthesised peptides. For smaller peptides $(<2200)$ the instrument used was an Agilent 1100 series MS and the column used was a Phenomenex Gemini C18 (150 x 3mm) column. The spectrometer was set at positive mode. For larger peptides (>2200), a Bruker Q-Tof, MicroTof-QII fitted with an electrospray source was used. Peptides were analysed in positive mode. ${ }^{1} \mathrm{H}$ NMR analysis was done using a Bruker Avance III 400 MHZ NMR. Infrared spectra were obtained on a Perkin Elmer spectrum 100 instrument with an attenuated total reflectance (ATR) attachment.

Peptides were synthesised manually or with the CEM Liberty automated peptide synthesiser. Table 12 indicates which peptides were synthesised manually and with the CEM Liberty automated peptide synthesiser. 
Table 12: Peptides sequences synthesised.

\begin{tabular}{|c|c|c|}
\hline Peptide sequence & Manual synthesis & Automated synthesis \\
\hline C-(D-Ala) 35 & & $\mathrm{X}$ \\
\hline C-D-A $\mathbf{A}_{4} \mathbf{G}_{5}$ & & $\mathrm{X}$ \\
\hline G-D-AFK & $\mathrm{X}$ & \\
\hline CK-D-AFKRKCoum & & $\mathrm{X}$ \\
\hline C(K-D-AFKRK $)_{3}$ Coum & & $\mathrm{X}$ \\
\hline CK-D-AFKRK & & $X$ \\
\hline C(K-D-AFKRK $)_{3}$ & & $X$ \\
\hline CG-D-AKFRGG & & $\mathrm{X}$ \\
\hline CKCoum & & $X$ \\
\hline
\end{tabular}

\subsubsection{Manual peptide synthesis}

The peptide G-D-AFK was synthesised manually at room temperature using the standard FmocSPPS procedures. Chlorotrityl resin was used to allow conjugation of the peptide to a longer sequence.

\section{Preparation of chlorotrityl resin}

Chlorotrityl resin (1.05 g) was placed in a $50 \mathrm{~mL}$ round bottom flask and suspended in approximately $20 \mathrm{~mL}$ of dry DCM. Thionyl chloride $(1.2 \mathrm{eq}, 139 \mu \mathrm{L})$ and piperidine $(2.4 \mathrm{eq}, 309$ $\mu \mathrm{L}$ ) was added to the flask. The mixture was refluxed for two to four hours under nitrogen, after which the DCM was removed and the resin was washed six times with dry DCM $(5 \mathrm{~mL})$.

\section{Attachment of first amino acid}

The resin was transferred to the fritted-filter sintered glass reaction vessel and washed six times with dry DCM (5 mL). Dry DCM (10 mL), DIEA (3 eq, $4.8 \mathrm{mmol}, 834 \mu \mathrm{L})$ and Fmoc-L-Cys(Trt)$\mathrm{OH}$ (3 eq, $4.8 \mathrm{mmol}, 1.9 \mathrm{~g}$ ) was added to the funnel and the mixture was bubbled under nitrogen for two hours. After attachment of the amino acid, the resin was washed three times with DMF (10 $\mathrm{mL} \cdot \mathrm{g}^{-1}$ of resin) to remove any unreacted amino acid. 


\section{Deprotection of the attached Fmoc-amino acid}

For the removal of the Fmoc protecting group from the attached amino acids, a 20\% (v/v) piperidine in DMF solution was used and bubbled under a stream of nitrogen for 15 minutes. After deprotection, the resin was washed five times with DMF $\left(10 \mathrm{~mL} \cdot \mathrm{g}^{-1}\right.$ of resin) to remove remaining piperidine.

\section{Determination of resin loading}

The removal of the Fmoc protecting group leads to the production of a benzofulvene derivative that is easily characterised by an intense absorbance. A mass of $1.2 \mathrm{mg}$ of the resin was weighed out and deprotected using $3 \mathrm{~mL} 20 \%$ piperidine (v/v) in DMF for two to three minutes. The solution of $20 \%$ piperidine was used as a blank. The absorbance was measured at $290 \mathrm{~nm}$ and the loading was determined using Equation 1.

$$
\frac{\mathrm{Abs}_{\text {Sample }}-\mathrm{Abs}_{\text {Blank }}}{1.65 \times \mathrm{mg} \text { of resin }}
$$

\section{Equation 1}

Where $\mathrm{Aabs}_{\text {sample }}$ is the absorbance of the sample, mg is the weight of the resin, $\mathrm{Abs}_{\text {blank }}$ is the $20 \%$ piperidine solution in DMF.

\section{The ninhydrin test ${ }^{140}$}

This test was used to determine the completion of the acylation reaction during the coupling of an amino acid and to monitor the deprotection of the Fmoc protecting group. Three solutions are used in this test and were prepared as described.

Preparation of solutions:

- $\mathrm{KCN}(16.5 \mathrm{~g})$ was dissolved in $25 \mathrm{~mL}$ distilled water. From the $25 \mathrm{~mL} \mathrm{KCN}$ solution, $1 \mathrm{~mL}$ was removed and diluted with $49 \mathrm{~mL}$ pyridine,

- Ninhydrin ( $1 \mathrm{~g})$ was dissolved in $20 \mathrm{~mL}$-butanol,

- Phenol (40 g) was dissolved in $20 \mathrm{~mL}$ n-butanol.

The ninhydrin test was carried out after each coupling and deprotection step. Approximately 10-15 resin beads were removed from the funnel after deprotection or coupling step. Three drops of each solution was added to the resin beads and heated for five minutes to $85^{\circ} \mathrm{C}$ in a water bath. No 
colour change was observed, the solution remained a faint yellow and resin beads remained clear, in the absence of free amino groups indicating complete coupling of amino acid to resin. After deprotection, free amino acid groups were present and this was indicated by a dark blue colour. The test thus yielded a negative result in the case of complete coupling and a positive result after complete deprotection.

\section{Attachment of consecutive amino acids}

The resin was washed six times with DMF after deprotection $(5 \mathrm{~mL})$. To $10 \mathrm{~mL}$ DMF the amino acid (4 eq), HBTU (3.6 eq), DIEA (3 eq) were added and the solution was bubbled under nitrogen for two hours. After attachment of the amino acid the resin was washed three times with DMF (10 $\mathrm{mL} \cdot \mathrm{g}^{-1}$ of resin) to remove any unreacted amino acid.

\section{Cleavage of the peptide from the resin}

Prior to cleavage, the DMF was drained and the resin washed thoroughly with dry DCM (20 mL. $\mathrm{g}^{-1}$ of resin). The peptide was cleaved from the resin using a solution of 5\% TFA (v/v), 92.5\% DCM $(\mathrm{v} / \mathrm{v})$ and $2.5 \%$ TIS (v/v) for two hours. The cleavage solution containing the peptide was collected and the resin washed with methanol $\left(20 \mathrm{~mL} \cdot \mathrm{g}^{-1}\right.$ of resin). The solvents of the combined filtrates were removed under reduced pressure.

\section{Peptide purification}

Analytical amounts of the peptide was dissolved in AcN:water and analyzed with LC-MS to identify the product peaks. The peptide was purified using a semi-preparative reverse phase high pressure liquid chromatography (RP-HPLC). The solvent gradient developed to allow for optimal separation of the crude peptide was $5-75 \%$ AcN in 20 minutes.

\section{Analysis of the purified peptide}

LC-MS was used to characterise the purified peptide. The spectrum is presented in Appendix 1 (page 139). 


\subsubsection{Automated peptide synthesis}

The remainder of the peptides synthesised (Table 12) were executed with the CEM LibertyAutomated microwave peptide synthesiser and the following conditions:

- Scale: $0.1 \mathrm{mmol}$,

- resin: Rink amide, $0.61 \mathrm{mmol} . \mathrm{g}^{-1}$,

- deprotection: $20 \%$ piperidine/DMF,

- coupling: HBTU:DIEA (0.5:1),

- coupling times: All amino acids (except arginine) - 15 minutes no microwave followed by 15 minutes microwave coupling. Arginine amino acid residues - 45 minutes no microwave followed by 15 minutes microwave coupling.

\section{Cleavage of the peptides from the resin}

All peptides were synthesised on rink amide resin. The peptides were cleaved from the resin using a solution of $95 \%$ TFA (v/v), 5\% thioanisole (v/v), 5\% phenol (v/v), 5\% distilled water and 2.5\% ethanedithiol $(\mathrm{v} / \mathrm{v})$ for two hours. The cleavage solution containing the peptide was collected and the resin washed with TFA $\left(20 \mathrm{~mL} \cdot \mathrm{g}^{-1}\right.$ of resin). The solvents of the combined filtrates were removed on a rotary evaporator to yield a thick, syrup-like substance, followed by the addition of 5 mL TFA and cold diethyl ether to precipitate the peptide. The white precipitate was then removed from the diethyl ether and TFA with a centrifuge (6000 RPM, 5 minutes), the supernatant discarded and the pellet redissolved in water and $\mathrm{AcN}$.

\section{Deprotection of $\mathrm{Cys}(\mathrm{tBu})$ protecting group using mercury (II) acetate ${ }^{79}$}

An amount of $0.1 \mathrm{mmol}$ of peptide was dissolved in $10 \mathrm{~mL}$ HPLC grade deoxygenated water. The $\mathrm{pH}$ was adjusted to 4.0 with one drop dilute acetic acid. The solution was blanketed with nitrogen. A mass of $0.1 \mathrm{~g}$ of mercury acetate was dissolved in $10 \mathrm{~mL}$ HPLC grade deoxygenated water and blanketed with nitrogen. The peptide and the mercury acetate solutions were mixed together and reacted at room temperature for 90 minutes. A volume of $0.5 \mathrm{~mL}$ of $\beta$-mercaptoethanol was added to the reaction mixture and heated at $45-50^{\circ} \mathrm{C}$ for 60 minutes. The reaction was allowed to continue for a further 60 minutes to ensure completion. The mixture was vacuum filtered through a mediumporosity sintered glass funnel containing celite ${ }^{\circledR}$ rinsed with deoxygenated water. The filtrate was evaporated. The residue was suspended in HPLC-grade water and evaporation was repeated three times to ensure the complete removal of $\beta$-mercaptoethanol. The product was analysed by LC-MS. 


\section{Peptide purification}

Analytical amounts of the peptides were dissolved in AcN:water and analyzed with LC-MS to determine the peptide purity. For the successful peptides (Table 12), the purity was quite high due to the use of extended coupling time (15 minutes no microwave, 15 minutes microwave coupling). As a result, it was not necessary to purify the peptides using semi-preparative RP-HPLC. Solid phase extraction (SPE) was carried out on the peptides to ensure the complete removal of the thiol present in the cleavage mixture as the thiol would interfere with the coupling of the peptides to the gold surface. For SPE, a LC-18 cartridge was conditioned with $4 \mathrm{~mL} \mathrm{AcN}$ and equilibrated with 3 $\mathrm{mL}$ water prior to use. Peptide stock solution $(0.5 \mathrm{~mL})$ was loaded and the flow-through collected and combined with a wash fraction of water $(1 \mathrm{~mL} \mathrm{x} 4)$. The fractions containing the peptide were lyophilised and stored as the dry powder.

\section{Peptide characterization}

The purified peptides were characterised by high resolution mass spectrometry, in which a LC was connected to a Bruker Q-Tof, MicroTof-QII, fitted with an electrospray source. The peptides were analysed with ${ }^{1} \mathrm{H}$ NMR and IR spectrometry.

\subsubsection{Synthesis of cysteine methyl ester Coumarin}

Coumarin-3-carboxylic acid ( $0.00263 \mathrm{~mol}, 0.5 \mathrm{~g})$ and oxalyl chloride $(0.0263 \mathrm{~mol}, 2.23 \mathrm{~mL})$ were added to a $100 \mathrm{~mL}$ round bottom flask in $10 \mathrm{~mL}$ DCM and stirred. Since Coumarin-3-carboxylic acid is not soluble in DCM, a few drops of DMF were added to ensure complete solubilisation of the fluorophore. The mixture was allowed to react for 24 hours under a stream of nitrogen. After this time, CME $(0.00789 \mathrm{~mol}, 1.35 \mathrm{~g})$ and thriethylamine $(0.013 \mathrm{~mol}, 1832.5 \mu \mathrm{L})$ were added to the solution and it was left to stir under nitrogen for 1 hour, after which, the solvent was removed using a high pressure vacuum pump. The contents of the flask were dissolved in ethyl acetate and extracted with $10 \% \mathrm{HCl}$. The product was then washed several times with brine and the ethyl acetate was removed under pressure. The final product was purified using flash silica chromatography and characterised using ${ }^{1} \mathrm{H}$ NMR. 


\subsection{GNP SYNTHESIS}

All reagents and solvents were purchased commercially and used unless otherwise stated without further purification. $\mathrm{HAuCl}_{4}$ was sponsored by Rand Refinery South Africa. Sodium citrate and $\mathrm{NaBH}_{4}$ were purchased from Merck.

Unbound peptides were removed by ultrafiltration, using VivaSciences Vivaspin tubes (MWCO $10,000)$ on a 1000 RPM Eppendorf Centrifuge $5415 \mathrm{C}$ and purification was performed three times against water. UV-vis absorption spectra were recorded on a Perkin Elmer Lambda 35 UV-vis spectrophotometer. Infrared spectra were obtained on a Perkin Elmer spectrum 100 instrument with an ATR attachment. ${ }^{1} \mathrm{H}$ NMR spectra of samples were obtained in $\mathrm{D}_{2} \mathrm{O}$ using a Bruker Avance III 400 MHZ NMR. TEM measurements were made on a Joel CX100 transmission electron microscope at a voltage of $100 \mathrm{~V}$. The samples for TEM were prepared by placing a drop of the colloidal solution onto carbon-coated ( $300 \AA)$ formvar films on copper grids. Samples were dried at room temperature before images were obtained. An Eppendorf Centrifuge 5415C was used prior to LC-MS analysis to remove traces of NPs after trypsin digestion. The Agilent series 1100 LC-MS was used to determine breakdown products after trypsin digestion.

\subsubsection{Synthesis of citrate coated GNPs}

All glassware used in the following preparations was washed thoroughly in a bath of freshly prepared aqua regia $\left(\mathrm{HNO}_{3}: \mathrm{HCl}=3: 1\right)$, rinsed with distilled water several times and placed in the oven overnight prior to use. A volume of $100 \mathrm{~mL}$ of a $1 \mathrm{mM}$ aqueous solution of $\mathrm{HAuCl}_{4}$ was brought to a vigorous boil with constant stirring in a round bottom flask fitted with a reflux condenser. At the time of boiling, a $10 \mathrm{~mL}$ of a $38.8 \mathrm{mM}$ aqueous solution of sodium citrate was rapidly added to the vortex of the solution. The previously yellow solution changed to wine red in a few seconds. Boiling was continued for a further 10 minutes after which the heating mantle was removed and the solution was stirred for a further 30 minutes. Once the solution had cooled to room temperature it was stored in a dark glass bottle in a refrigerator. The size of the GNPs were verified by TEM analysis with an average size of $15 \mathrm{~nm}$ (average of about 300 particles). The surface plasmon resonance band of the NP solution at $\sim 520 \mathrm{~nm}$ (depending on extent of aggregation) was determined with UV-vis spectrophotometry. The particle concentration was $5.5 \mathrm{x}$ $10^{-7} \mathrm{M}$ according to Beers law using a molar absorbtivity of $2.7 \times 10^{8} \mathrm{M}^{-1} \mathrm{~cm}^{-1}$ at $520 \mathrm{~nm}$ (Equation 2). ${ }^{141}$ 


$$
\begin{aligned}
\mathbf{A} & =\sum \mathbf{c L} \\
\mathbf{c} & =\frac{\mathbf{A}}{\sum \mathbf{L}}
\end{aligned}
$$

Where $A=1.48$

$$
\sum=2.7 x 10^{8} \mathrm{M}^{-1} \mathrm{~cm}^{-1}
$$

$$
\mathbf{L}=\mathbf{1} \mathbf{c m}
$$

$$
\begin{gathered}
c=\frac{1.48}{2.7 \times 10^{8} \mathrm{M}^{-1} \mathrm{~cm}^{-1} \times 1 \mathrm{~cm}} \\
c=5.5 \times 10^{-9} \mathrm{M}
\end{gathered}
$$

\section{Equation 2}

\subsubsection{Attachment of peptides to sodium citrate stabilised GNPs}

Under vigorous stirring, $10 \mu \mathrm{L}$ of $10 \mathrm{mM}$ aqueous peptide solution was added to $10 \mathrm{~mL}$ of a $5 \mathrm{nM}$ aqueous GNPs solution. On addition of the peptide to the original red NP solution, the solution turned light blue with a visible black precipitate. The stirring was continued for 24 hours. Excess sodium citrate and unreacted peptide were removed from solution by repeated cycles of high-speed centrifugation and redispersion was attempted in water. Attachment of the following peptide sequences was attempted in various ratios: CKCoum, CMECoum, CGAFKGGCoum, CKAFKRKCoum $\mathrm{C}(\mathrm{KAFKRK})_{3}$ and $\mathrm{C}(\mathrm{KAFKRK})_{3}$ Coum. The products were characterised using UV-vis spectrophotometry, fluorescence spectrophotomery, ATR-FTIR spectrometry and ${ }^{1} \mathrm{H}$ NMR.

\subsubsection{Synthesis of NPs using the sodium borohydride reduction method}

All glassware used in the following preparations was washed thoroughly in a bath of freshly prepared aqua regia $\left(\mathrm{HNO}_{3}: \mathrm{HCl}=3: 1\right)$, rinsed several times with distilled water and placed in the oven overnight prior to use. To $3 \mathrm{~mL}$ of a $0.02 \mathrm{mmol}$ aqueous solution of $\mathrm{HAuCl}_{4}, 1 \mathrm{~mL}$ of a $0.02-$ $0.12 \mathrm{mmol}^{\mathrm{p}}$ aqueous solution of peptide (various peptides were attached to the GNPs as shown in

\footnotetext{
$\mathrm{p}$ The amount of thiol was varied in order to determine the amount of thiol that yielded the desired GNP size as shown in Section 4.3.8. The number of moles selected was $0.02 \mathrm{mmol}$.
} 
Table 13, page 117) was added and the yellow solution stirred for 20 minutes. The reduction of gold was carried out by the dropwise addition of $0.5 \mathrm{~mL}$ of $0.2 \mathrm{mmol}$ aqueous solution of icechilled $\mathrm{NaBH}_{4}$. The original yellow solution rapidly turned red/purple/black (depending on the peptide used, refer to Table 7 (page 58). The mixture was left for 24 hours, after which the solution was loaded into Vivaspin centrifuge tubes, centrifuged for an hour and washed in triplicate with water to ensure the complete removal of unreacted peptides. The peptide capped GNPs were then redissolved in double distilled water, lyophilised and stored as the dry powder. 


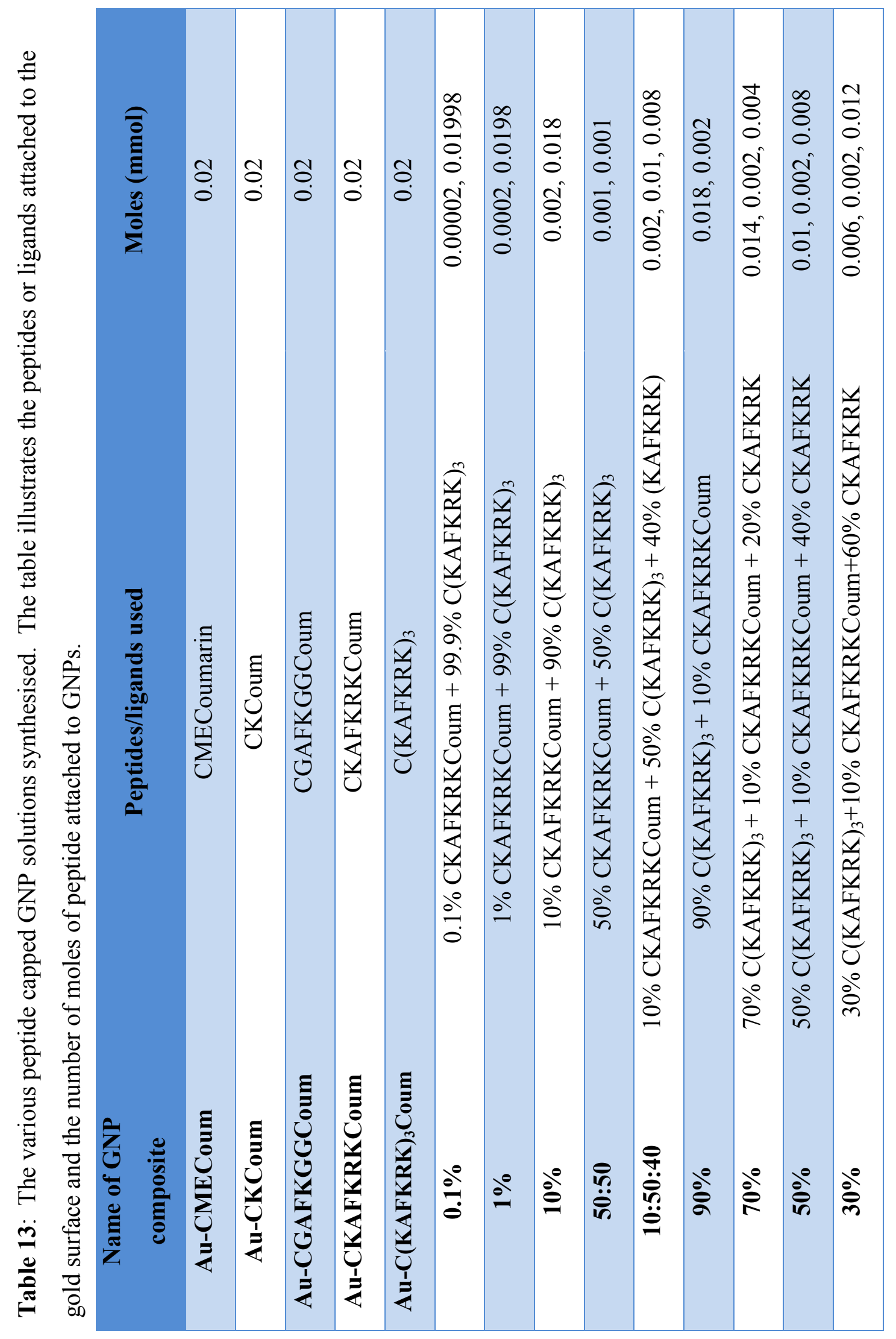




\subsubsection{Characterization of sodium borohydride reduced GNPs}

The functionalised GNPs were characterised with ${ }^{1} \mathrm{H}$ NMR, IR and UV-vis spectroscopy. TEM images of the GNPs were taken to determine the shape and size of the particles. The concentration of the functionalised GNPs was calculated using Equation 3 to Equation 5. ${ }^{\mathbf{4 0}}$

$$
N=\frac{\pi}{6} \frac{\rho D^{3}}{M}=30.896 D^{3}
$$

\section{Equation 3}

Where: $\mathrm{D}=$ The average core diameters of the particles in $\mathrm{nm}$,

$\mathrm{N}=$ The average number of gold atoms for each type of nanosphere,

$\rho \quad=$ The density for fcc gold $\left(19.3 \mathrm{~g} \cdot \mathrm{cm}^{-1}\right)$,

$\mathrm{M} \quad=$ Atomic weight of gold $\left(197 \mathrm{~g} \cdot \mathrm{mol}^{-1}\right)$.

$$
\mathbf{C}=\frac{\mathbf{N}_{\text {Total }}}{\mathbf{N V N}_{\mathbf{A}}}
$$

\section{Equation 4}

Where: $\mathrm{N}_{\text {total }}=$ Total number of gold atoms, equivalent to the initial amount of gold salt added to the reaction solution.

$\mathrm{N}=$ Average number of gold atoms per nanosphere,

$\mathrm{V}=$ Volume of reaction solution in $\mathrm{L}$,

$\mathrm{N}_{\mathrm{a}} \quad=$ Avagadro's number.

$$
\mathbf{A}=\boldsymbol{\varepsilon} \mathbf{C l}
$$

\section{Equation 5}

Where: $\varepsilon \quad=$ Molar absorbtivity,

$\mathrm{C}=$ Concentration,

$1=$ Length of the cuvette. 


\subsubsection{Determination of critical coagulation concentrations}

To determine the CCC of CKAFKRKCoum, C(KAFKRK $)_{3}$ Coum and $50 \% \mathrm{C}(\mathrm{KAFKRK})_{3}: 40 \%$ CKAFRKR:10\% CKAFKRKCoum passivated gold particles, a series of tubes containing $500 \mu \mathrm{L}$ of GNP solutions of the following concentrations were prepared:

- $0.23 \mathrm{nM}$ of CKAFKRKCoum,

- $0.4 \mathrm{nM}$ of $\mathrm{C}(\mathrm{KAFKRK})_{3} \mathrm{Coum}$,

- $\quad 0.24 \mathrm{pM}$ of $50 \% \mathrm{C}(\mathrm{KAFKRK})_{3}: 40 \%$ CKAFRKR:10\% CKAFKRKCoum.

Increasing volumes of a $1.7 \mathrm{M} \mathrm{NaCl}$ stock solution were added to each sample. Aggregation was assessed by monitoring changes in the characteristic GNP plasmon frequency (in the wavelength region of 400-700 nm). $\mathrm{NaCl}$ aliquots were added until the plasmon band was no longer detectable. At that point, further aggregation was no longer visible and the plasmon band did not shift to further wavelengths. The analyses were performed in triplicate for each GNP solution. The analysis for each GNP composite was done twice.

\subsection{FLUORESCENCE STUDIES}

All reagents and solvents were purchased commercially and unless otherwise stated, used without further purification. PBS buffer was from Lonza. The emission spectra were recorded using a Perkin Elmer Precisely LS 55 spectrometer between 390-550 nm upon excitation using $7.5 \mathrm{~nm} / 7.5$ $\mathrm{nm}$ (excitation/emission) slit widths. Disposable poly(methyl methacrylate) cuvettes, obtained from PlastiBrand, were used instead of the conventional fluorescence quartz cuvettes, in order to prevent memory effects from previous analyses. 
The solutions prepared for the fluorescence studies were freshly prepared in $1 \mathrm{x}$ PBS buffer (pH 7). GNPs solutions were prepared by weighing out $\sim 1 \mathrm{mg}$ of the solid peptide capped GNP and dissolving the solid in $10 \mathrm{~mL}$ of 1 x PBS buffer. The absorbance of the solution was measured and adjusted to the same value for a particular set of analyses. The concentrations of the solutions were determined based on the absorbance. Three GNPs solutions were analysed, namely Au-CKCoum, Au-CKAFKRKCoum and Au-C(KAFKRK) $)_{3}$ Coum. Three separate tests were performed:

- A comparison of the fluorescence intensity of CKCoum, Au-CKAFKRKCoum and Au$\mathrm{C}(\mathrm{KAFKRK})_{3}$ Coum to evaluate the effect the length of the peptide has on the fluorescence intensity,

- a comparison of the fluorescence intensity of $0.1 \%, 1 \%$ and $10 \%$,

- a comparison of the fluorescence intensity of 10\% CKAFKRKCoum:50\% $\mathrm{C}(\mathrm{KAFKRK})_{3}: 40 \%$ CKAFKRK before and after trypsin/versene exposure.

Prior to fluorescence studies the absorbance of each GNP composite was analysed and adjusted to a known value, thereby ensuring a constant concentration for each test.

\subsection{TRYPSIN/VERSENE DIGESTION}

All reagents and solvents were purchased commercially and used unless otherwise stated without further purification. Trypsin/versene mixture $(0.25 \% \mathrm{w} / \mathrm{v}$ trypsin $)$ was from Lonza.

Trypsin digests were carried out on the peptides and peptide capped GNPs. The peptide $\mathrm{C}(\mathrm{KAFKRK})_{3}$ was analysed to establish the main fragment of cleavage. A $500 \mathrm{mg} \cdot \mathrm{L}^{-1}$ peptide solution was prepared in PBS solution. A sterile Eppendorf tube was used for digestion, into which $1.5 \mathrm{~mL}$ of the $500 \mathrm{mg} . \mathrm{L}^{-1}$ solution and $15 \mu \mathrm{L}$ trypsin/versene was added, the solution was vortexed thoroughly, to ensure proper mixing, and placed in an incubator at $37^{\circ} \mathrm{C}$ overnight. A blank solution containing only $1.5 \mathrm{~mL}$ of the peptide solution was also incubated in a separate Eppendorf tube and incubated under the same conditions. After incubation, the mixtures were centrifuged (1000 RPM, five minutes), the supernatant removed and analysed using LC column chromatography connected to a Bruker Q-Tof, MicroTof-QII, fitted with an electrospray source.

After the main cleavage peak was established, the functionalised GNP solutions were analysed to determine whether cleavage of the peptides attached to GNPs was possible. The procedure was followed for the digestion of the GNPs as with the $\mathrm{C}(\mathrm{KAFKRK})_{3}$ peptide. The concentration of the GNPs was calculated from the absorbance value at $520 \mathrm{~nm}$ (Equation 3, Equation 4 and Equation 5) 
and it was ensured that all the concentrations be the same for comparison purposes. Each digestion was done in triplicate.

The Au-peptide composites analysed were:

- $100 \%$ Au-CKAFKRKCoum,

- $100 \% \mathrm{Au}-\mathrm{C}(\mathrm{KAFKRK})_{3} \mathrm{Coum}$,

- $\left.\mathrm{Au}-[50 \% \mathrm{CKAFKRKCoum:50 \%} \mathrm{C(KAFKRK})_{3}\right]$,

- $\mathrm{Au}-\left[50 \% \mathrm{C}(\mathrm{KAFKRK})_{3}: 40 \%\right.$ CKAFKRK:10\% CKAFKRKCoum].

The same generalised protocol was followed for all subsequent trypsin digestion experiments.

\subsubsection{Loading experiment}

As shown in section 5.6.4. (page 80) four functionalised GNP solutions were synthesised for loading experiments: $90 \%, 70 \%, 50 \%$ and $30 \%$. The GNP powders were dissolved in $5 \mathrm{~mL}$ PBS in a sterile centrifuge tube. The concentrations were determined by measurement of absorbance at 520 $\mathrm{nm}$ (Equation 3 to Equation 5) and were ensured to all be the same value. Trypsin/versene was added to the GNP solutions $(50 \mu \mathrm{L})$ and the solutions thoroughly vortexed. as a control GNP solutions of the same concentrations were prepared without the addition of trypsin/versene. The solutions were incubated for 3, 6, 9 and 24 hours. After each time point, the solutions were analysed using LC connected to a Bruker Q-Tof, MicroTof-QII, fitted with an electrospray source to determine the cleavage products. Triplicate analyses were carried out on each GNP solution.

\subsection{CELL CULLTURE}

\subsubsection{Growth and maintenance of cell lines}

Cryopreserved A549 and MDBK cells were received from the Department of Biochemistry, University of KwaZulu-Natal (Westville campus). The laminar flow cabinet comprised of a VividAir laminar hood. All reagents and solvents were purchased commercially and unless otherwise stated used without further purification. Heat-inactivated foetal calf serum (FCS) was from Invitrogen. Dulbeccoes minimal essential medium (DMEM) with 4.5 g. $\mathrm{L}^{-1}$ glucose and Lglutamine, RPMI 1640 with 25 mM 2-[4-(2-hydroxyethyl)-1-piperazinyl] ethanesulfonic acid (HEPES) and L-glutamine, penicillin/streptomycin (pen strep) mixture, trypsin/versene mixture $(0.25 \% \mathrm{w} / \mathrm{v}$ trypsin) and PBS were purchased from Lonza. Tissue culture apparatus was supplied by Griener Bio-one. A CellTiter 96 non-radioactive cell proliferation kit was supplied by Promega. 
Cryopreservation of cells was performed using Nalgene Cryo $1{ }^{\circ} \mathrm{C}$ freezing container. A Brightline haemocytometer from Hausser Scientific and a Nikon TMS inverted microscope was used for cell counting and analysis. Absorbance readings for the MTT assay were performed using an Automated Microplate Reader (ELx800) from Bio-Tek Instruments.

Aseptic techniques ${ }^{\mathrm{q}}$ were used consistently when working with all cell types. Prior to any cell culture work, the laminar flow was sterilized by UV radiation for an hour, followed by thorough ethanol swabbing. FCS, DMEM, trypsin/versene, pen strep and PBS were removed from the refrigerator and placed in a sterilized water bath adjusted to $37^{\circ} \mathrm{C}$.

Reconstituted DMEM was prepared by supplementing DMEM with $2.5 \mathrm{M}$ HEPES, to give a final concentration of $25 \mathrm{nM}$ and pen strep mixture to give a final concentration of $0.1 \mathrm{mg} \cdot \mathrm{mL}^{-1}$. DMEM was also supplemented with $10 \%$ and $5 \%(\mathrm{v} / \mathrm{v})$ heat-inactivated FCS for maintenance of the A549 and MDBK cell lines respectively.

Vials containing cells for reconstitution were removed from storage in liquid nitrogen and thawed by rapid agitation in a $37^{\circ} \mathrm{C}$ water bath for approximately five minutes. Immediately upon thawing, vials were sterilised with $70 \%(\mathrm{v} / \mathrm{v})$ ethanol, opened aseptically and the contents transferred to a 10 $\mathrm{mL}$ corning centrifuge tube. Cells were pelleted by low speed centrifugation and resuspended in 10 $\mathrm{mL}$ fully constituted DMEM supplemented with either $5 \%$ or $10 \%$ FCS (depending on the cell type). The cell suspension was equally distributed between the surface of two $75 \mathrm{~cm}^{2}$ flasks containing $10 \mathrm{~mL}$ fully constituted DMEM [supplemented with either $5 \%$ or $10 \%$ FCS (depending on the cell type)] to yield a 1:2 subculture or split. Flasks were placed in a sterile incubator at a constant temperature of $37^{\circ} \mathrm{C}$ and allowed to reach confluency ${ }^{\mathrm{r}}$.

Subsequent subculturing was carried out on cells near confluence. Growth medium was decanted and cell monolayers were washed with $10 \mathrm{~mL}$ of PBS. After removal of the washing solution, 1.5 $\mathrm{mL}$ trypsin/versene solution was added to the monolayer until cells were observed (using an inverted microscope) to round up. The trypsin/versene solution was immediately removed, the culture vessel tapped with the palm of the hand, and $10 \mathrm{~mL}$ fully constituted DMEM supplemented

\footnotetext{
q Aseptic or sterile techniques is the execution of tissue culture protocols without introducing microorganism contamination from the environment. Such special precautions includes working in a laminar flow hood near a flame, swabbing all equipment with ethanol and using only autoclaved consumables.

${ }^{\mathrm{r}}$ Confluence refers to the extent to which cells cover the tissue culture flask. $100 \%$ confluency means that the entire surface of the tissue culture flask is completely occupied by cells (no more space for cells to grow).
} 
with either $5 \%$ or $10 \%$ FCS (depending on the cell type) was added to the dislodged cells. The cells were evenly dispersed by repeated aspiration (up to 15 times) into a sterile $5 \mathrm{~mL}$ disposable plastic pipette. The cell suspension was again evenly distributed between two new $75 \mathrm{~cm}^{2}$ culture flasks containing fully constituted DMEM (supplemented with either $5 \%$ or $10 \%$ FCS depending on the cell type) to give a 1:2 dilution. Flasks were placed in a sterile incubator at a constant temperature of $37^{\circ} \mathrm{C}$ and allowed to reach confluency.

Cells of $80 \%$ confluency were cryopreserved. Cultures were washed with PBS, trypsinised ${ }^{\mathrm{s}}$ and removed from the flask surface (as described above). The dislodged cells were evenly distributed in $2 \mathrm{~mL}$ fully constituted DMEM supplemented with either 5\% or 10\% FCS (depending on the cell type) and transferred to a $2 \mathrm{~mL}$ corning cryogenic vial. The cryoprotectant dimethylsulfoxide (DMSO) was added $(10 \% \mathrm{v} / \mathrm{v})$ and the cryogenic vials immediately subjected to cooling at a steady state of $1^{\circ} \mathrm{C}$ per minute to approximately $-70^{\circ} \mathrm{C}$. Once completely frozen, vials were transferred to liquid nitrogen at $-196^{\circ} \mathrm{C}$.

\subsubsection{MTT assay}

Fully constituted RPMI 1640 was prepared by supplementing RPMI 1640 with 2.5 M HEPES, to give a final concentration of $25 \mathrm{nM}$, and pen strep mixture to give a final concentration of 0.1 mg.mL ${ }^{-1}$. Fully constituted RPMI 1640 supplemented with 10\% FCS was used for the MTT assay and will be referred to as RPMI 1640 in the remainder of the dissertation.

Cells were trypsinised as described in section 7.5.1. (page 121) and resuspended in RPMI 1640. Cell counts were carried out such that each $100 \mu \mathrm{L}$ of cell solution contains $5 \times 10^{4}$ cells. After counting, cells were plated into 96-well tissue culture plates and incubated at $37^{\circ} \mathrm{C}$ for six hours to ensure complete attachment of the cells to the tissue culture wells.

The sensitivity of the cells to cadmium was elucidated to ensure the validity of the test protocol and technique. Cadmium chloride samples $(40 \mu \mathrm{L})$ at various concentrations $(0.0017-0.044 \mathrm{M})$ were prepared in sterile water and RPMI 1640 media. After six hours, the culture media was removed from each well of the culture plate and $40 \mu \mathrm{L}$ of cadmium sample and $60 \mu \mathrm{L}$ of fresh RPMI 1640 added to each well. The plates were sealed and incubated at $37^{\circ} \mathrm{C}$ for 42 hours.

\footnotetext{
${ }^{\mathrm{s}}$ Trypsinization is the process whereby cells adhering to the surface of a tissue culture flask are lifted from the surface of the culture vessel using a trypsin/versene solution.
} 
After the incubation period, cell viability was assessed as per the manufacturer ${ }^{\text {ee }}$ instructions, using the CellTiter 96 non-radioactive cell proliferation assay. In short, $15 \mu \mathrm{L}$ of the dye solution was added to each well and the plate incubated at $37^{\circ} \mathrm{C}$ for three hours, after which solubilisation solution $(100 \mu \mathrm{L})$ was added to each well and the plate incubated for an hour at $37^{\circ} \mathrm{C}$ with gentle agitation. The plate was then taken to the plate reader to measure absorbance.

The absorbance maximum for formazan is at $570 \mathrm{~nm}$ and a wavelength of $690 \mathrm{~nm}$ was selected to reduce any background absorbance that could be the result of cell debris or fingerprints. The plate reader was programmed to give a final reading derived by subtraction of an optical density (OD) reading at $570 \mathrm{~nm}$ from the reading at $690 \mathrm{~nm}$, at various intervals, to determine the optimal time that is required for solubilisation of the formazan crystals.

Cytotoxicity was calculated as follows:

$$
\begin{gathered}
\text { MTT reduction }(\% \text { of control })=\frac{\text { OD of sample }}{\text { OD of control }} \times 100 \% \\
\text { Percent Cytotoxicity }=100-\text { MTT reduction }
\end{gathered}
$$

A total of four control assays were performed in order to ascertain an acceptable level of reproducibility between assays.

To determine the sensitivity of the cells to GNPs, cells were prepared and plated at a cell density of $5 \times 10^{4}$ cells per well. Various concentrations of the GNPs $\left(2.09 \times 10^{-11}\right.$ to $\left.9.2 \times 10^{-11} \mathrm{M}\right)$ were prepared in water and RPMI 1640 and added to the respective wells to determine the cytotoxic effect on the MDBK and A549 cell lines. The cell preparation and test protocol were as described previously. The GNP assays were performed in triplicate. 


\subsection{TEM AND CONFOCAL MICROSCOPY}

TEM measurements were made on a Joel CX100 transmission electron microscope at a voltage of $100 \mathrm{kV}$. The sample for TEM was prepared by placing a drop of the colloidal solution onto carboncoated $(300 \AA)$ formvar films on copper grids. Samples were dried at room temperature before images were obtained. The microscope used was a Zeiss 710 (Germany) CFSM (excitation at wavelength $366 \mathrm{~nm}$ and emission at wavelength $460 \mathrm{~nm}$ ). 24-well tissue culture plates used for TEM and confocal microscopy were from Nunc. Circular thermanox cover slips for TEM use were obtained from Nunc and circular glass coverslips for confocal microscopy were from Lasec.

\subsubsection{TEM}

The plating protocol for TEM is similar to that described in section 7.5.2. (page 123), although, due to the difference in dimension of the 24-well tissue culture plate well and the 96-well tissue culture plate, $1.75 \times 10^{5}$ cells per $100 \mu \mathrm{L}$ were used for the 24 -well plate as opposed to the $5 \times 10^{4}$ cells per $100 \mu \mathrm{L}$ used in the 96-well plates. A volume of $500 \mu \mathrm{L}$ of cell solution was added to each well and was allowed to incubate at $37^{\circ} \mathrm{C}$ for 30 minutes to ensure attachment of cells to coverslip, after which $1 \mathrm{~mL}$ of RPMI 1640 media was added and allowed to incubate for a further six hours. After incubation and media removal, $600 \mu \mathrm{L}$ of fresh RPMI 1640 media was added along with $400 \mu \mathrm{L}$ of GNP compound prepared in water and media $\left(2.09 \times 10^{-11} \mathrm{M}\right)$ (as described in section 7.5.2., page 123). The plates were sealed and incubated for 42 hours at $37^{\circ} \mathrm{C}$. After the incubation period the media was removed from all the wells. Sufficient gluteraldehyde was added to cover the entire cell surface and left for half an hour (primary fixation). Phosphate buffer was used to wash the cells (3 x 5 minutes), after which $0.5 \%$ osmium tetroxide was added and left to stand for one hour (postfix). The cells were washed with phosphate buffer ( $3 \times 5$ minutes) and the cells were dehydrated with increasing percentages of acetone:

- 2 x 5 minutes $30 \%$ acetone,

- 2 × 5 minutes $50 \%$ acetone,

- 2 x 5 minutes $70 \%$ acetone,

- 2 x 10 minutes $100 \%$ acetone.

The next phase carried out was infiltration, whereby cells were covered by equal parts of resin and acetone and allowed to stand for four hours. After four hours, the acetone:resin mixture was removed and the cells covered with whole resin and placed on a turn-table overnight. The resin was polymerized - the specimen was orientated in a mould, covered with whole resin and placed in an oven for eight hours at $70^{\circ} \mathrm{C}$. Samples were then removed from the mould and sliced using a 
Microtome to the appropriate sizes for subsequent TEM analyses. The assay was carried out on three separate occasions to ensure complete reproducibility (three different 24-well plates were prepared). On each occasion, three wells were embedded with resin and two images were captured per coverslip.

\subsubsection{Confocal microscopy}

The plating protocol for confocal analyses is similar to that described in section 7.5.2. (page 123) although, due to the difference in dimension of the 24-well tissue culture plate well and the 96-well tissue culture plate, $1.75 \times 10^{5}$ cells per $100 \mu \mathrm{L}$ were used for the 24 -well plate as opposed to the 5 $\mathrm{x} 10^{4}$ cells per $100 \mu \mathrm{L}$ used in the 96 -well plates. A volume of $500 \mu \mathrm{L}$ of cell solution was added to each well and was allowed to incubate at $37^{\circ} \mathrm{C}$ for 30 minutes to ensure attachment of cells to coverslip, after which $1 \mathrm{~mL}$ of RPMI 1640 media was added and allowed to incubate for a further six hours. After incubation and media removal, $1000 \mu \mathrm{L}$ of fresh RPMI 1640 media was added. The plates were then sealed and incubated for 42 hours at $37^{\circ} \mathrm{C}$ to ensure complete confluency of the cells.

The following coverslips were prepared:

- Three wells containing cells only (control one),

- three wells containing cells and unfunctionalised GNPs (control two),

- twelve wells containing cells and functionalised GNPs $\left(2.09 \times 10^{-11} \mathrm{M}\right)$.

Prior to analyses of the control slides the cells were washed three times with PBS. The coverslip was mounted (cell side down) onto a clean glass microscope slide, covered with a thin glass slip and sealed with nail polish. The sample slide was placed on the stage with the sample side down.

Three wells containing functionalised GNPs were incubated for one minute, three hours, four hours, and 24 hours. After the respective time periods, the cells were washed three times with PBS to ensure complete removal of free GNPs. The coverslip was mounted (cell side down) onto a clean glass microscope slide, covered with a thin glass slip and sealed with nail polish. The sample slide was placed on the stage with the sample side down.

For each assay, three replicates were done (three coverslips were used). Approximately five images were captured per coverslip to ensure a representative image was obtained. Different areas of the coverslip were imaged to prevent the occurrence of bleaching of the fluorophore. This prevented the introduction spurious artefacts that could be the result of handling. An oil immersion was used 
for the objective lens to increase the resolution of the microscope. All parameters, such as pinhole and brightness, were held at a constant value throughout the testing. 


\section{REFERENCES}

1. Fayed, L., What Is Cancer? Understanding What Cancer Is and How Cancer Develops, http://cancer.about.com/od/newlydiagnosed/a/whatcancer.htm, (2009), 1 October 2009

2. Kumar, K., Selvaraj, V., Alagar, M., Synthesis of PET-PLA/Drug Nanoparticles and Their Effect with Gold Nanoparticles for Controlled Drug Release in Cancer Chemotherapy, Research Letters in Nanotechnology, (2008), 1-4

3. Drake, E., 50 Plus One Questions to Ask Your Doctor, Encouragement Press, (2006)

4. Cancer Institute, Cancer causes and risk factors, http://www.cancer.gov/cancertopics/prevention-genetics-causes/causes, (2009), 15 April 2009

5. de Groot, F., Broxterman, H., Adams, H., van Vliet, A., Tesser, G., Elderkamp, Y., Schra, A., Kok, R., Molema, G., Pinedo, H., Scheeren, H., Design, Synthesis, and Biological Evaluation of a Dual Tumour Specific Motive Containing Integrin-targeted Plasmin Cleavable Doxorubicin Prodrug, Molecular Cancer Therapeutics, (2002), 1, 901-911

6. Brigger, I., Dubernet, C., Couvreur, P., Nanoparticles in Cancer Therapy and Diagnosis, Advanced Drug Delivery Reviews, (2002), 54, 631-651

7. Denny, W., Prodrug Strategies in Cancer Therapy, European Journal of Medicinal Chemistry, (2001), 36, 577-595

8. Carl, P., Chakravarty, P., Katzenellenbogen, J., Weber, M., Protease-Activated "Prodrugs" for Cancer Chemotherapy, The Proceedings of the National Academy of Sciences, USA, (1980), 77, 2224-2228

9. Wikipedia, Antigen, http://en.wikipedia.org/wiki/Antigen, (2009), 15 July 2009

10. Pan, C., Cardarelli, P., Nieder, M., Pickford, M., Gangwar, S., King, D., Yarranton, G., Buckman, D., Roscoe, W., Zhou, F., Sales, M., Chen, T., Horgan, K., Wang, Y., Nguyen, T., Bebbington, C., CD10 Is a Key Enzyme Involved in the Activation of Tumour-activated Peptide Prodrug CPI-0004Na and Novel Analogues: Implications for the Design of Novel Peptide Prodrugs for the Therapy of CD10+ Tumours, Cancer Research, (2003), 63, 55265531

11. Hosta, L., Pla-Roca, M., Arbiol, J., Lo'pez-Iglesias, C., Samitier, J., Cruz, L., Kogan, M., Albericio, F., Conjugation of Kahalalide F with Gold Nanoparticles to Enhance in Vitro Antitumoral Activity, Bioconjugate Chemistry, (2009), 20, 138-146

12. Chace, D., Sparkman, O., What is Mass Spectrometry? The Importance of Communicating the Concept of Mass Spectrometry to Professionals, Media, and the Consumer, http://www.asms.org/portals/0/WhatIsMSPoster.pdf, (2009), 
13. Hutchings, G., Brust, M., Schmidbaur, H., Gold - An Introductory Perspective, Chemical Society Reviews, (2008), 37, 1759-1765

14. Hutchings, G., Brust, M., Schmidbaur, H., The Lycurgus Cup - A Roman Nanotechnology, Gold Bulletin, (2007)

15. Ofir, Y., Samanta, B., Rotello, V., Polymer and Biopolymer Mediated Self-Assembly of Gold Nanoparticles, Chemical Society Reviews, (2008), 37, 1814-1825

16. Triulzi, R., Dai, R., Zou, J., Leblanc, R., Gud, Q., Orbulescu, J., Huob, Q., Photothermal Ablation of Amyloid Aggregates by Gold Nanoparticles, Colloids and Surfaces B: Biointerfaces, (2008), 63, 200-208

17. Cormode, D., Skajaa, T., Fayad, Z., Mulder, W., Nanotechnology in Medical Imaging: Probe Design and Applications, Arteriosclerosis, Thrombosis, and Vascular Biology, (2009), 29, 1-14

18. Shukla, R., Bansal, V., Chaudhary, M., Basu, A., Bhonde, R., Sastry, M., Biocompatibility of Gold Nanoparticles and their Endocytotic Fate inside the Cellular Compartment: A Microscopic Overview, Langmuir, (2005), 21, 10644-10654

19. Connor, E., Mwamuka, J., Gole, A., Murphy, C., Wyatt, M., Gold Nanoparticles are Taken up by Human Cells but do not Cause Acute Cytotoxicity, Small, (2005), 1, 325-327

20. Mackiewicz, M., Ayres, B., Reed, S., Reversible, Reagentless Solubility Changes in Phosphatidylcholine-Stabilized Gold Nanoparticles, Nanotechnology (2008), 19, 1-6

21. Chandler, J., Gurmin, T., Robinson, N., The Place of Gold in Rapid Tests, http://www.devicelink.com/ivdt/archive/00/03/004.html, (2009), 30 September 2009

22. Kosmella, S., Polyelectrolytes and Nanoparticles, Springer, (2007)

23. Sugunan, A., Dutta, J., Nanoparticles for Nanotechnology, Journal of Physics Science and Idea, (2004), 4, 50-57

24. Schulz-Dobrick, M., Sarathy, K., Jansen, M., Surfactant-Free Synthesis and Functionalization of Gold Nanoparticles, Journal of the American Chemical Society, (2005), 127, 12816-12817

25. Warren, C., Chan, W., Bio-Applications of Nanoparticles, Volume 620 of Advances in Experimental Medicine and Biology, Illustrated ed.; Springer, (2007)

26. Zhang, J., Badugu, R., Lakowicz, J., Fluorescence Quenching of CdTe Nanocrystals by Bound Gold Nanoparticles in Aqueous Solution, Plasmonics (2008), 3, 3-11

27. Colorado, R., Lee, T., Thiol-based Self-assembled Monolayers: Formation and Organization, (2001)

28. Wikipedia, Colloidal gold, http://en.wikipedia.org/wiki/Colloidal_gold, (2009), 20 July 2009 
29. Hu, M., Chen, J., Li, Z., Au, L., Hartland, G., Li, Z., Marqueze, M., Xia, Y., Gold Nanostructures: Engineering their Plasmonic Properties for Biomedical Applications, Chemical Society Reviews, (2006), 35, 1084-1094

30. Demberelnyam, D., Ariuna, M., Shim, Y., Newly Synthesized Water Soluble CholiniumPurpurin Photosensitizers and Their Stabilized Gold Nanoparticles as Promising Anticancer Agents, International Journal of Molecular Sciences, (2008), 9, 864-871

31. Daniel, M., Astruc, D., Gold Nanoparticles: Assembly, Supramolecular Chemistry, Quantum-Size-Related Properties, and Applications toward Biology, Catalysis, and Nanotechnology, Chemical Reviews, (2004), 104, 293-346

32. Levy, R., Thanh, N., Doty, R., Hussain, I., Nichols, R., Schiffrin, D., Brust, M., Fernig, D., Rational and Combinatorial Design of Peptide Capping Ligands for Gold Nanoparticles, Journal of the American Chemical Society, (2004), 126, 10076-10084

33. Pasquato, L., Pengo, P., Scrimin, P., Functional Gold Nanoparticles for Recognition and Catalysis, Journal of Materials Chemistry, (2004), 14, 3481-3487

34. Pengo, P., Polizzi, S., Battagliarin, M., Pasquato, L., Scrimin, P., Synthesis, Characterization and Properties of Water-Soluble Gold Nanoparticles with Tunable Core Size, Journal of Materials Chemistry, (2003), 13, 2471-2478

35. Sugunan, A., Thanachayanont, C., Dutta, J., Juilland, P., Hilborn, J., Synthesis of BioCompatible Gold Nanoparticles, (2002).

36. Wangoo., N., Bhasin., K, Mehtab., S, Suri., R, Synthesis and Capping of Water-Dispersed Gold Nanoparticles by an Amino Acid: Bioconjugation and Binding Studies, Journal of Colloid and Interface Science, (2008), 323, 247-254

37. Bhattacharya, S., Srivastava, A., Synthesis of Gold Nanoparticles Stabilised by MetalChelator and the Controlled Formation of Close-Packed Aggregates by Them, Indian Academy of Sciences, (2003), 115, 613-619

38. Myroshnychenko, V., Rodri'guez-Ferna' ndez, J., Pastoriza-Santos, I., Funston, A., Novo, C., Mulvaney, P., Liz-Marza' n, L., Garci'a de Abajo, F., Modelling the Optical Response of Gold Nanoparticles, Chemical Society Reviews, (2008), 37, 1792-1805

39. Huang, X. PhD thesis, Georgia Institute of Technology, (2006).

40. Liu, X., Atwater, M., Wang, J., Huo, Q., Extinction Coefficient of Gold Nanoparticles with Different Sizes and Different Capping Ligands, Colloids and Surfaces B: Biointerfaces, (2007), 58, 3-7

41. Fuente, J., Berry, C., Tat Peptide as an Efficient Molecule to Translocate Gold Nanoparticles into the Cell Nucleus, Bioconjugate Chemistry, (2005), 16, 1176-1180 
42. Noponen, V., Bhat, S., Sievänen, E., Kolehmainen, E., Novel two-step synthesis of gold nanoparticles capped with bile acid conjugates, Materials Science and Engineering $C$ (2008), 28, 1144-1148

43. Mukherjee, P., Bhattacharya, R., Bone, N., Lee, Y., Patra, C., Wang, S., Lu, L., Secreto, C., Banerjee, P., Yaszemski, M., Kay, N., Mukhopadhyay, D., Potential Therapeutic Application of Gold Nanoparticles in B-Chronic Lymphocytic Leukaemia (BCLL): Enhancing Apoptosis, Journal of Nanobiotechnology (2007), 5, 1-13

44. Grace, A., Pandian, K., Antibacterial Efficacy of Aminoglycosidic Antibiotics Protected Gold Nanoparticles - A Brief Study, Colloids and Surfaces A: Physicochemistry Engineering Aspects, (2007), 279, 63-70

45. Shenoy, D., Fu, W., Li, J., Crasto, C., Jones, G., Dimarzio, C., Sridhar, S., Amiji, M., Surface Functionalization of Gold Nanoparticles using Hetero-Bifunctional Poly(ethylene glycol) Spacer for Intracellular Tracking and Delivery, International Journal of Nanomedicine, (2006), 1, 51-57

46. Aumelas, A., Serrero, A., Durand, A., Dellacherie, E., Leonard, M., Nanoparticles of Hydrophobically Modified Dextrans as Potential Drug Carrier Systems, Colloids and Surfaces B: Biointerfaces, (2007), 59, 74-80

47. Duchesne, L., Gentili, G., Comes-Franchini, M., Fernig, D., Robust Ligand Shells for Biological Applications of Gold Nanoparticles, Langmuir, (2008), 24, 13572-13580

48. Higuchi, M., Ushiba, K., Kawaguchi, M., Structural Control of Peptide-Coated Gold Nanoparticle Assemblies by the Conformational Transition of Surface Peptides, Journal of Colloid and Interface Science, (2007), 308, 356-363

49. Fan, J., Chen, S., Gao, S., Coating Gold Nanoparticles with Peptide Molecules via a Peptide Elongation Approach, Colloids and Surfaces B: Biointerfaces, (2003), 28, 199-207

50. Aryal, S., Remant, C., Dharmaraj, N., Bhattarai, N., Kim, C., Kim, H.,, Spectroscopic Identification of S Au Interaction in Cysteine Capped Gold Nanoparticles, Spectrochimica Acta Part A, (2006), 63, 160-163

51. Shalom, D., Wootton, R., Winkle, R., Cottam, B., Vilar. R., deMello, A., Wilde, P., Synthesis of Thiol Functionalized Gold Nananoparticles using a Continuous Flow Microfluidic Reactor, Materials Letters (2007), 61, 1146-1150

52. Bailey, P., An Introduction to Peptide Chemistry; John Wiley and Sons, (1990)

53. Benoiton, N., Chemistry of Peptide Synthesis; CRC Press, (2005)

54. Sewald, N., Jakubke, H., Peptides: Chemistry and Biology; Wiley-VCH Verlag GmbH \& Co, (2002) 
55. Albericio, F., Carpino, L., Coupling Reagents and Activation, Methods in Enzymology, (1997), 1-25

56. Han, S., Kim, A., Albericio, F., Humphrey, J., Chamberlin, R., Strategies for Peptide Synthesis: An Overview,

http://chemgroups.northwestern.edu/scheidt/PDFs/2004_pdfs/04_Pokorsi_AmidePres.pdf, (2004), 20 April 2009

57. Law, H., The Organic Chemistry of Peptides; John Wiley and Sons, (1970)

58. Chan, W., White, P., Fmoc Solid Phase Peptide Synthesis: A Practical Approach; Oxford University Press, (2000), 346 pages

59. Jensen, H., Bioorganic Chemistry - Lecture Notes on Peptide Synthesis, http://www.chem.au.dk/ www/weeknotes/week_2347.pdf., (2007), 20 June 2009

60. Chemistry, Organic Synthesis in Drug Discovery, http://userwww.sfsu.edu/ cberkman/CHEM_640_synth_part_1.pdf, (2009), 20 May 2009

61. Bhagavan, N., Medical biochemistry; Academic Press, (2000)

62. Bodansky, M., Peptide Chemistry: A Practical Textbook; Liberty of Congress, (1988)

63. Bodansky, M., Klausner, Y., Ondetti, M., Peptide Synthesis; John Wiley and Sons, (1966),195 pages

64. Barrett, G., Elmore, D., Amino Acids and Peptides; Cambridge University Press, (1998)

65. Bodansky, M., Principles of Peptide Synthesis, (1993); Vol. 16

66. Hermanson, G., Bioconjugate Techniques; Academic Press, (2008), 1202 pages

67. Lloyd-Williams, P., Albericio, F., Giralt, E., Chemical Approaches to the Synthesis of Peptides and Proteins. New Directions in Organic and Biological Chemistry, Illustrated Revised ed.; CRC Press, (1997), 278 pages

68. Hockfield, S., Selected Methods for Antibody and Nucleic Acid Probes, Illustrated ed.; CSHL Press, (1993)

69. Kappe, C., Dallinger, D., Murphree, S., Practical Microwave Synthesis for Organic Chemists: Strategies, Instruments, and Protocols; Wiley-VCH, (2009)

70. Howl, J., Peptide Synthesis and Applications; Humana Press, (2005)

71. Sigma Aldrich, Custom Peptide Synthesis, http://www.sigmaaldrich.com/lifescience/custom-oligos/custom-peptides/learning-center/solid-phase-synthesis.html, (2009), 3 July 2009

72. Tracy, J., Crowe, M., Hampe, O., Felds-Zinna, C., Dass, A., Murray, R., Electrospray Ionization Mass Spectrometry of Uniform and Mixed Monolayer Nanoparticles: $\mathrm{Au} 25[\mathrm{~S}(\mathrm{CH} 2) 2 \mathrm{Ph}] 18$ and $\mathrm{Au} 25[\mathrm{~S}(\mathrm{CH} 2) 2 \mathrm{Ph}] 18-\mathrm{x}(\mathrm{SR}) \mathrm{x}$, Journal of the American Chemical Society, (2007), 129, 16209-16215 
73. Yonezawa, T., Sutoh, M., Kunitake, T., Practical Preparation of Size-Controlled Gold Nanoparticles in Water, The Chemical Society of Japan, (1997), 619-620

74. Brust, M., Walker, M., Bethell, D., Schiffrin, D., Whyman, R., Synthesis of Thiolderivatised Gold Nanoparticles in a Two-phase Liquid-Liquid System, Journal of the American Chemical Society, (1997), 801-802

75. Aslan, K., P'erez-Luna, V., Quenched Emission of Fluorescence by Ligand Functionalized Gold Nanoparticles, Journal of Fluorescence, (2004), 14, 401-404

76. Storhoff, J., Elghanian, R., Mirkin, C., Letsinger, C., Sequence-Dependent Stability of DNA-Modified Gold Nanoparticles, Langmuir, (2002), 18, 6666-6670

77. Biosciences, E., Products for Peptide Synthesis, (2008), 13 April 2009

78. McCurdy, S., Applied Biosystems User Bulletin Peptide Synthesizer, (1989)

79. Biosystems, A., Cleavage, Deprotection, and Isolation of Peptides after Fmoc Synthesis, (1998)

80. Pastuszak, J., Chimiak, A., Tert-Butyl Group as Thiol Protection in Peptide Synthesis, Journal of the American Chemical Society, (1981), 46, 1868-19873

81. Huang, C., Yang, Z., Lee, K., Chang, H., Synthesis of Highly Fluorescent Gold Nanoparticles for Sensing Mercury(II), Angewandte Chemical International Edition, (2007), 46, 6824-6828

82. Bechinger, B., Membrane Insertion and Orientation of Polyalanine Peptides: A 15N SolidState NMR Spectroscopy Investigation, Biophysical Journal, (2001), 81, 2251-2256

83. Salamone, J., Polymeric Materials Encyclopaedia, CRC Press, (1996)

84. Warrass, R., Wieruszeski, J., Boutillon, C., Lippens, G., High-Resolution Magic Angle Spinning NMR Study of Resin-Bound Polyalanine Peptides, Journal of the American Chemical Society, (2000), 122, 1789-1795

85. Shang, L., Qin, C., Wang, T., Wang, M., Wang, L., Dong, S., Fluorescent Conjugated Polymer-Stabilized Gold Nanoparticles for Sensitive and Selective Detection of Cysteine, Journal of Physical Chemistry, (2007), 111, 13414-13417

86. Tullman, J., Finney, W., Lin, Y., Bishnoi, S., Tunable Assembly of Peptide-coated Gold Nanoparticles, Plasmonics (2007), 2, 119-127

87. Bruch, M., NMR spectroscopy techniques; CRC Press, (1996), 616 pages

88. Aryal, S., Bhadur, R., Bhattarai, N., Kim, C., Kim, H., Study of Electrolyte Induced Aggregation of Gold Nanoparticles Capped by Amino Acids, Journal of Colloidal and Interface Science, (2006), 299, 191-197 
89. Storhoff, J., Elghanian, R., Mucic, R., Mirkin, C., Letsinger, R., One-Pot Colorimetric Differentiation of Polynucleotides with Single Base Imperfections using Gold Nanoparticle Probes, Journal of the American Chemical Society, (1998), 120

90. Somasundaran, P., Encyclopedia of Surface and Colloid Science, Volume 7; Somasundaran, P., Ed.; CRC Press, (2006).

91. Salamone, J., Concise Polymeric Materials Encyclopaedia; CRC Press, (1999)

92. Kissa, E., Dispersions: Characterization, Testing, and Measurement; CRC Press, (1999)

93. Royer, C., Approaches to Teaching Fluorescence Spectroscopy, Biophysical Journal (1995), 68, 1191-1195

94. Oswego, Fluorescence spectroscopy, www.oswego.edu/.../CHE425L/FLUORESCENCE_SPECTROSCOPY 08.pdf (2008), 23 September 2009

95. Lichtman, J., Conchello, J., Fluorescence Microscopy, Nature Methods, (2005), 2, 910-919

96. Cazes, J., Encyclopaedia of Chromatography: Den New Dekker Encyclopaedias; CRC Press, (2001)

97. Levine, B., Principles of Forensic Toxicology American Association for Clinical Chemistry, (2003)

98. Chasteen, T., Relaxation Mechanism for Excited State Molecules, http://elchem.kaist.ac.kr/vt/chem-ed/quantum/jablonsk.htm, (2006), 19 September 2009

99. Sawyer, L., Grubb, D., Meyers, G., Polymer microscopy, (1987)

100. Invitrogen, Technical Focus: Fluorescence Resonance Energy Transfer (FRET)-Note 1.2, http://www.invitrogen.com/site/us/en/home/References/Molecular-Probes-TheHandbook/Technical-Notes-and-Product-Highlights/Fluorescence-Resonance-EnergyTransfer-FRET.html, (2009), 16 September 2009

101. Wikipedia, Trypsin, http://en.wikipedia.org/wiki/Trypsin, (2009), 15 September 2009

102. Gaurise, C., Pasquato, L., Filippis, V., Scrimin, P., Gold Nanoparticles-Based Protease Assay, Proceedings of the National Academy of Science, (2006), 103, 3978-3982

103. Lee, S., Cha, E., Park, K., Lee, S., Hong, J., Sun, I., Kim, S., Choi, K., Kwon, I., Kim, K., Ahn, C., A Near-Infrared-Fluorescence-Quenched Gold-Nanoparticle Imaging Probe for In Vivo Drug Screening and Protease Activity Determination, Angewandte Chemical International Edition, (2008), 47, 2804-2807

104. Zhena, S., Fang, Y., Huang, C., Long, Y., Tryptophan-Contained Peptide-Functional Nanomaterials as general Spectrofluorometric Ragents for Enzyme, Talanta (2008), 76, 230-232 
105. Wikipedia, Mass Spectrometry, http://en.wikipedia.org/wiki/Mass_spectrometry, (2009), 17 September 2009

106. Van Bramer, S., An Introduction to Mass Spectrometry, (1998)

107. Arazona, U., http://www.chem.arizona.edu/massspec/intro html/intro.html, (2009), 13 September 2009

108. Dunn, B., Michael, W., Peptide Analysis Protocols; Humana Press, (1994); Vol. 36

109. Frokjaer, S., Hogvaard, L., Pharmaceutical Formulation Development of Peptides and Proteins; CRC Press, (2000)

110. Cioffi, N., De Palo, F., Calvano, C., Van der Werf, I., Palmisano, F., Core-Shell Gold Nanoparticles as Non-Conventional Matrix for the MALDI-ToF-MS Detection of Amino Acids: A Preliminary Study, Sensor Letters, (2008), 6, 654-661

111. Kim, Y., Oh, E., Shon, H., Moon, D., Lee, T., Kim, H., Gold Nanoparticle-Enhanced Secondary Ion Mass Spectrometry and its Bio-Applications, Applied Surface Science, (2008), 255, 1064-1067

112. Engle, J., Williams, S., Gold Nanoparticles as an Inorganic Matrix for MALDI-TOF Mass Spectrometry of Low Molecular Weight Polymers: Laboratory for Advance Separations Technologies, Department of Chemistry and Geochemistry, Colorado School of Mines, Golden, Colorado, (2008).

113. Kirk, J., Bohn, P., Surface Adsorption and Transfer of Organomercaptans to Colloidal Gold and Direct Identification by Matrix Assisted Laser Desorption/Ionization Mass Spectrometry, Journal of the American Chemical Society, (2003), 126, 5920-5926

114. Shimizu, H., Sakamoto, M., Nagahorib, N., Nishimura, S., A New Glycosylation Method. Part 2: Study of Carbohydrate Elongation onto the Gold Nanoparticles in a Colloidal Phase, Tetrahedron, (2007), 63, 2418-2425

115. Gies, D., Hercules, A., Gerdon, D., Cliffel, A., Electrospray Mass Spectrometry Study of Tiopronin Monolayer-Protected Gold Nanoclusters, Journal of the American Chemical Society, (2007), 129, 1095-1104

116. Redmond, C., Microsoft Encarta - Cytotoxicity, (2005).

117. Crouch, M., Slater, J., High-Throughput Cytotoxicity Screening: Hit and Miss., Drug discoveries and Therapeutics, (2001), 6, 48-53

118. Whitcutt, J., South African Journal of Science, (2005), 101, 383-388

119. Matsumoto, T., Phytochemistry Research Progress; Nova Science, (2008)

120. Weyermann, J., Lochmann, D., Zimmer, A., A Practical Note on the use of Cytotoxicity Assays, International Journal of Pharmaceutics, (2005), 288, 369-376 
121. Cook, J., Mitchell, J., Viability Measurements in Mammalian Cell Systems, Analytical Biochemistry, (1989), 179, 1-7

122. Rode, H., Eisel, D., Frost, I., In Apoptosis, Cell Death and Cell Proliferation Manual, Roche Applied Science (2007)

123. Wikipedia, Microscopy, http://en.wikipedia.org/wiki/Microscopy, (2009), 15 September 2009

124. Abramowitz, M., Microscope: Basics and Beyond; Olympus America Inc., Scientific Equipment Division, Two Corporate Centre Drive, Melville, (2003); Vol. 1

125. Wikipedia, Confocal Microscopy, http://en.wikipedia.org/wiki/Confocal_microscopy, (2009), 15 September 2006

126. Gibson, J., Khanal, B., Zubarev, E., Paclitaxel-Functionalized Gold Nanoparticles, Journal of the American Chemical Society (2007), 129, 11653-11661

127. Wikipedia, Electron Microscope, http://en.wikipedia.org/wiki/Electron_microscope, (2009), 15 September 2009

128. Breunig, M., Bauer, S., Goepferich, A., Polymers and Nanoparticles: Intelligent Tools for Intracellular Targeting?, European Journal of Pharmaceutics and Biopharmaceutics, (2008), 68, 112-128

129. Wikipedia, Transmission Electron Microscopy, http://en.wikipedia.org/wiki/Transmission_electron_microscopy, (2009), 15 September 2009

130. Wagner, B., The Use of Coumarins as Environmentally-Sensitive Fluorescent Probes of Heterogeneous Inclusion Systems, Molecules, (2009), 14, 210-237

131. Gu, C., Cheng, J., Lin, C., Lam, Y., Cheng, S. Wong, W., Nuclear Penetration of Surface Functionalized Gold Nanoparticles, Toxicology and Applied Pharmacology (2009), 237, 196-204

132. Tsai, S., Chen, Y., Liaw, J., Compound Cellular Imaging of Laser Scanning Confocal Microscopy by Using Gold Nanoparticles and Dyes, Sensors, (2008), 48, 2306-2316

133. Mandal., S., Selcakannan., P, Phadtare., S, Pasricha., R, Sastry., M, Synthesis of a Stable Gold Hydrosol by the Reduction of Chloroaurate Ions by the Amino Acid, Aspartic Acid, Proceedings of the Indian Academy of Sceince, (2002), 114, 513-520

134. Zhu, Z., Ghosh, P., Miranda, O., Vachet, R., Rotello, V., Multiplexed Screening of Cellular Uptake of Gold Nanoparticles Using Laser Desorption/Ionization Mass Spectrometry, Journal of the American Chemical Society, (2008), 130, 14139-14143

135. Nam, H., Kwon, S., Chung, H., Lee, S., Kwon, S., Jeon, H., Kim, Y., Park, J., Kim, J., Her, S., Oh, Y., Kwon, I., Kim, K., Jeong, S., Cellular Uptake Mechanism and Intracellular Fate 
of Hydrophobically Modified Glycol Chitosan Nanoparticles, Journal of Controlled Release, (2009), 135, 259-267

136. Muthusamy, K. MSc dissertation, University of KwaZulu-Natal, (2008).

137. Hatcher, E., Chen, Y., Kang, Y., Cadmium Resistance in A549 Cells Correlates with Elevated Glutathione Content but not Antioxidant Enzymatic Activities, Free Radical Biology and Medicine, (1995), 19, 805-812

138. Kim, T., Noh, M., Lee, H., Joo, S., Lee, S., Lee, K., Fluorescence-Based Detection of Point Mutation in DNA Sequences by CdS Quantum Dot Aggregation, Journal of Physical Chemistry, (2009)

139. Chen, W., Tu, X., Guo, X., Fluorescent Gold Nanoparticles-Based Fluorescence Sensor for $\mathrm{Cu}^{2+}$ Ions, Chemical Communication, (2009), 1736-138

140. Chemtech, A., Handbook of Combinatorial Organic and Peptide Chemistry; Advanced Chemtech, (2003).

141. Liua, Y., Liub, Y., Mernaugh, R., Zenga, X., Single Chain Fragment Variable Recombinant Antibody Functionalized Gold Nanoparticles for a Highly Sensitive Colorimetric Immunoassay, Biosensors and Bioelectronics 24, (2009), 2853-2857 


\section{APPENDIX 1: CHARACTERIZATION OF PEPTIDES}

Basic MS was carried out on the peptides not involved in the final application (proof of concept).

- GAFK

- CGAFKGGCoum

- CKCoum

- CMECoum

High-resolution MS was performed on all the peptides that were necessary for the use in the proof of concept.

- CKAFKRK

- CKAFKRKCoum

- $\mathrm{C}(\mathrm{KAFKRK})_{3}$

- $\mathrm{C}(\mathrm{KAFKRK})_{3} \mathrm{Coum}$ 


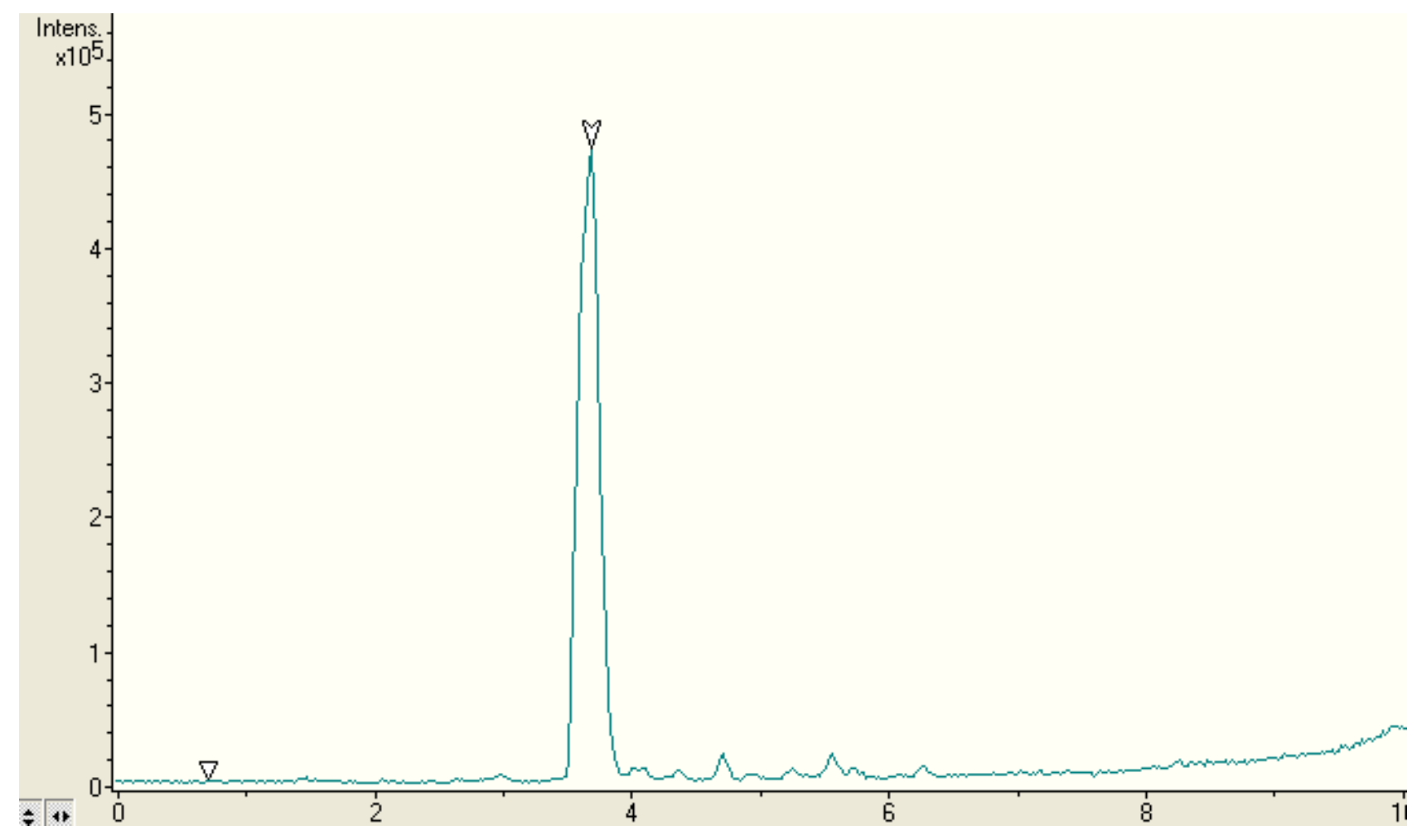

Chromatograph 1: LC-MS chromatograph of the peptide GAFK.

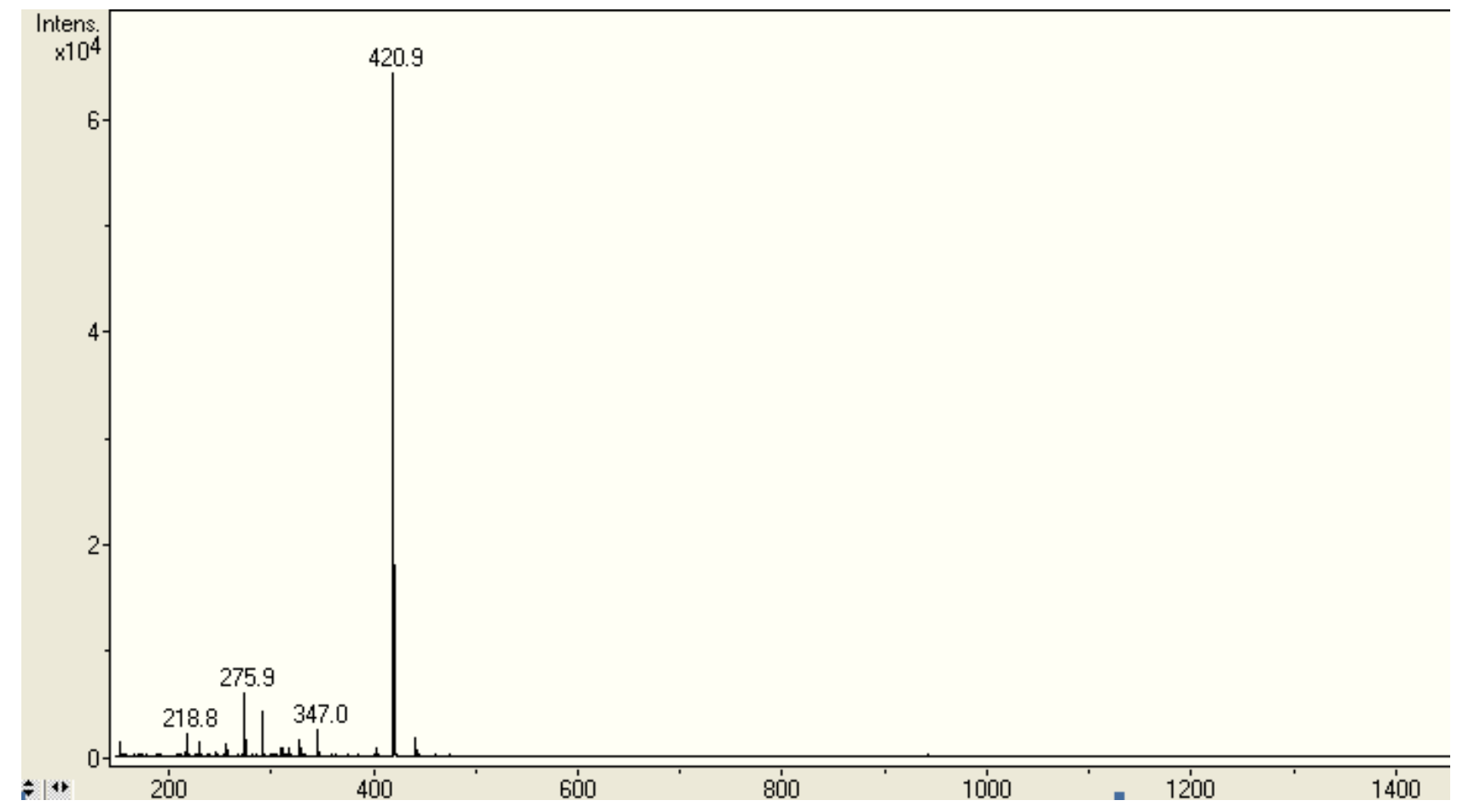

Spectrum 3: Mass spectrum of GAFK peptide. 


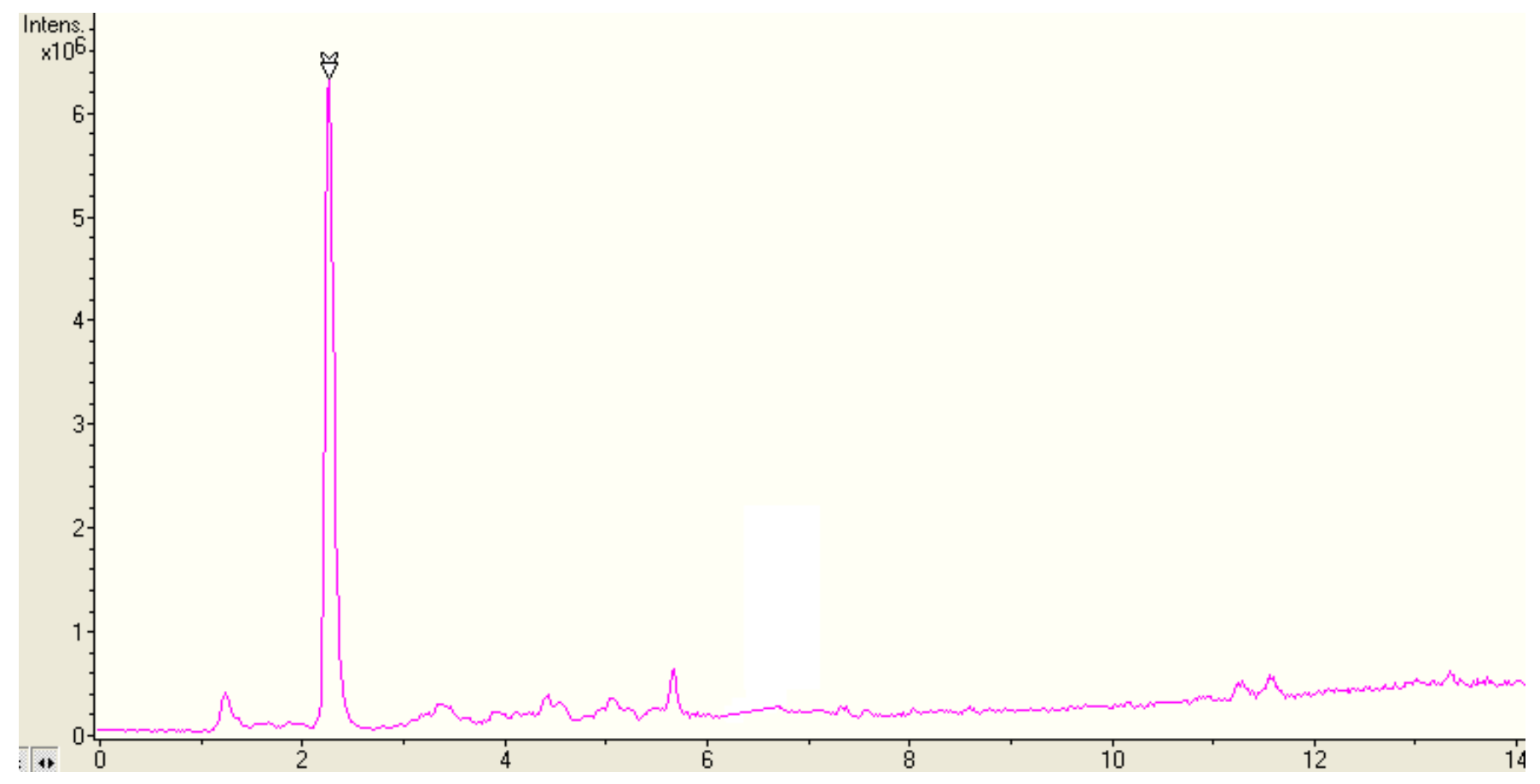

Chromatograph 2: LC-MS chromatograph of the peptide CKAFKRK.

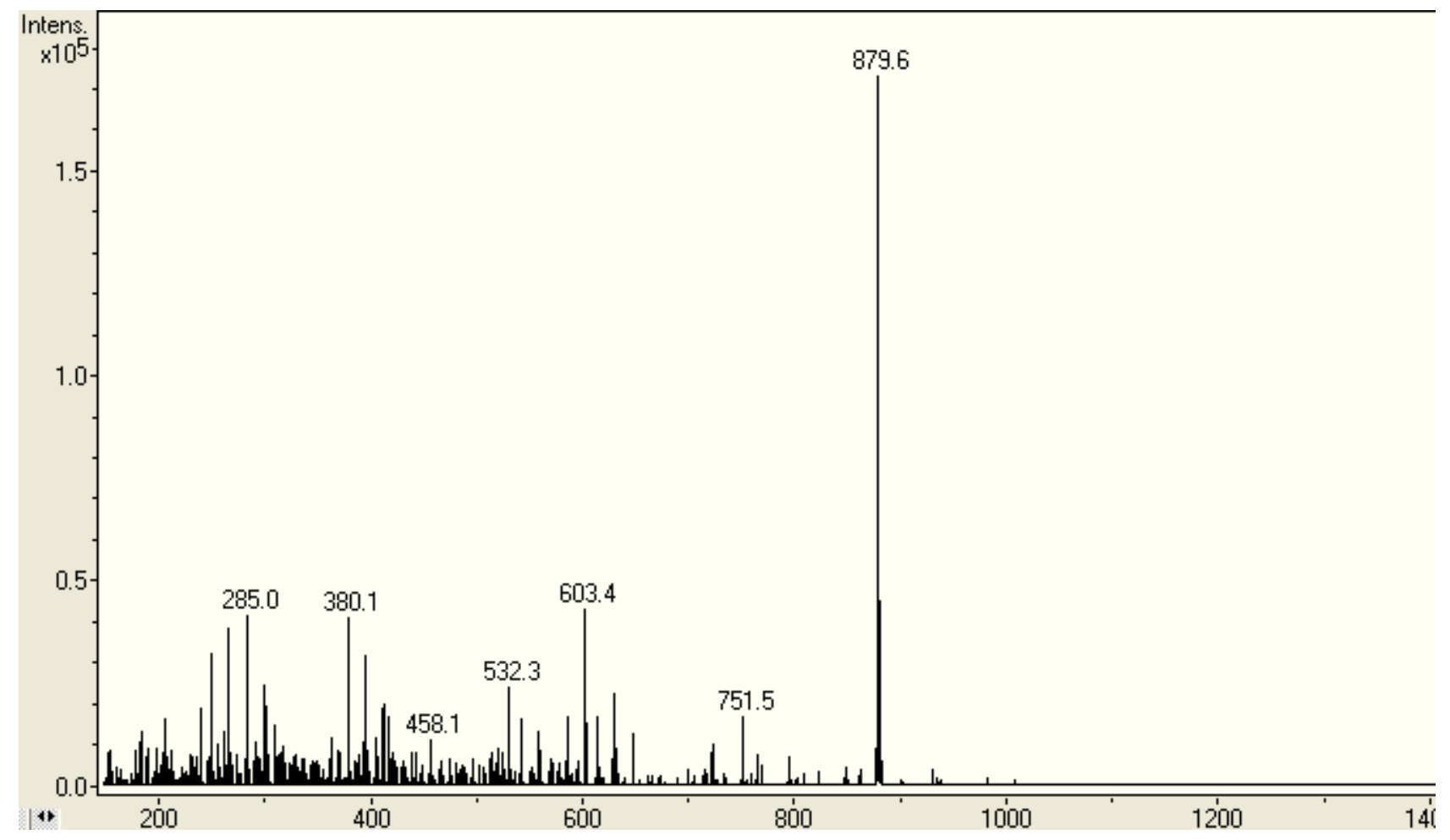

Spectrum 4: Mass spectrum of CKAFKRK peptide. 


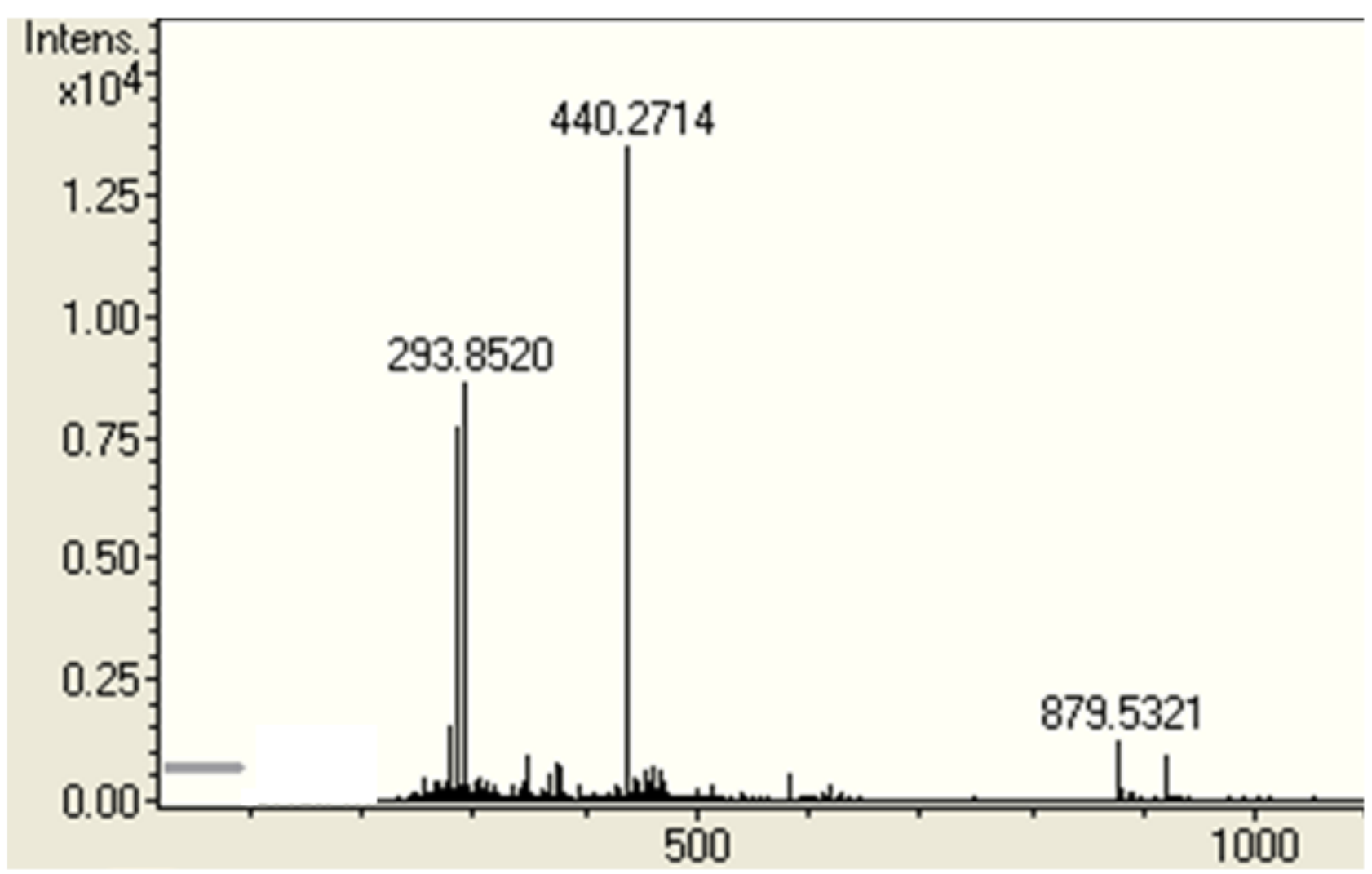

Spectrum 5: High resolution mass spectrum of peptide CKAFKRK.

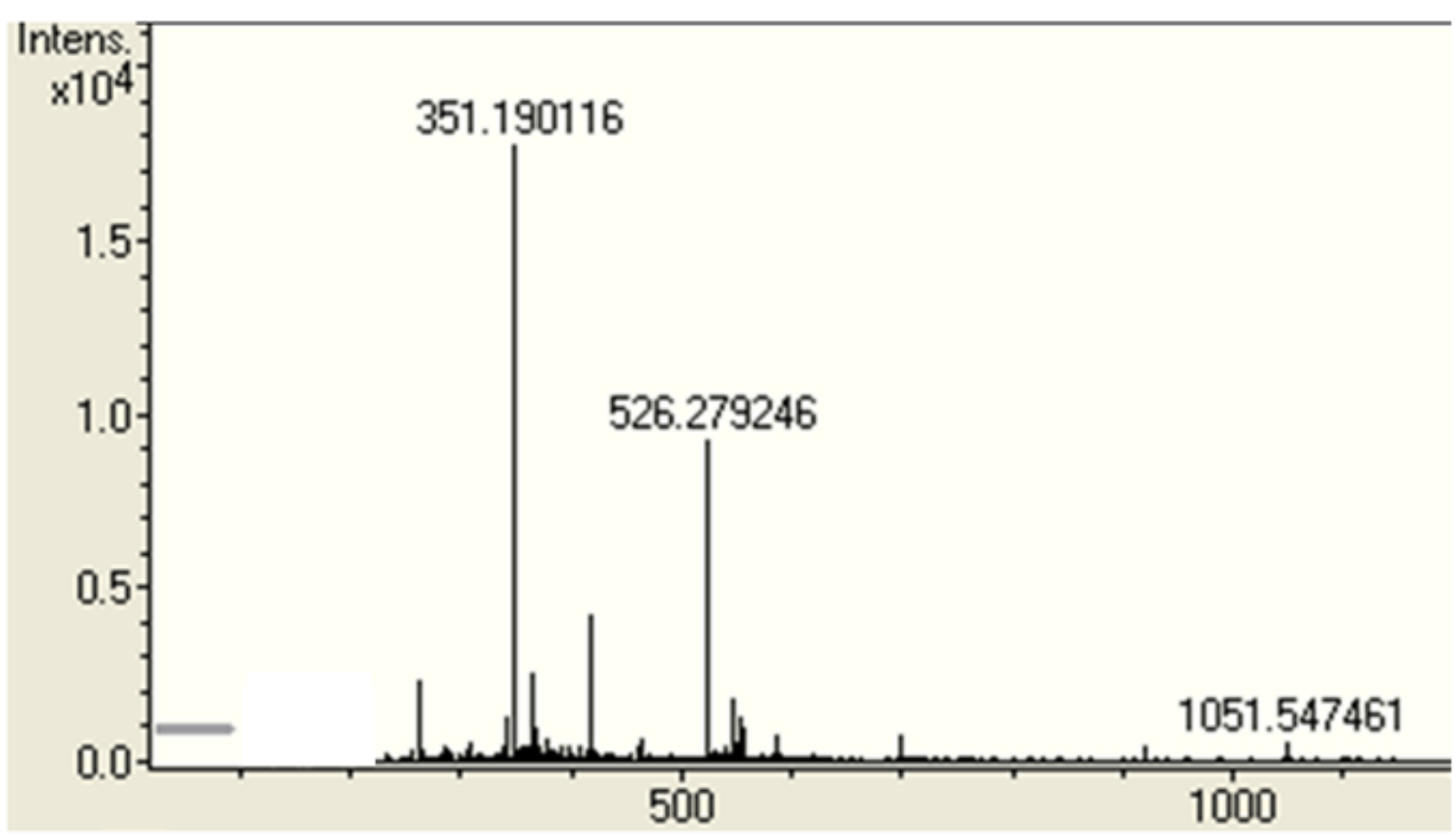

Spectrum 6: High resolution mass spectrum of peptide CKAFKRKCoum. 


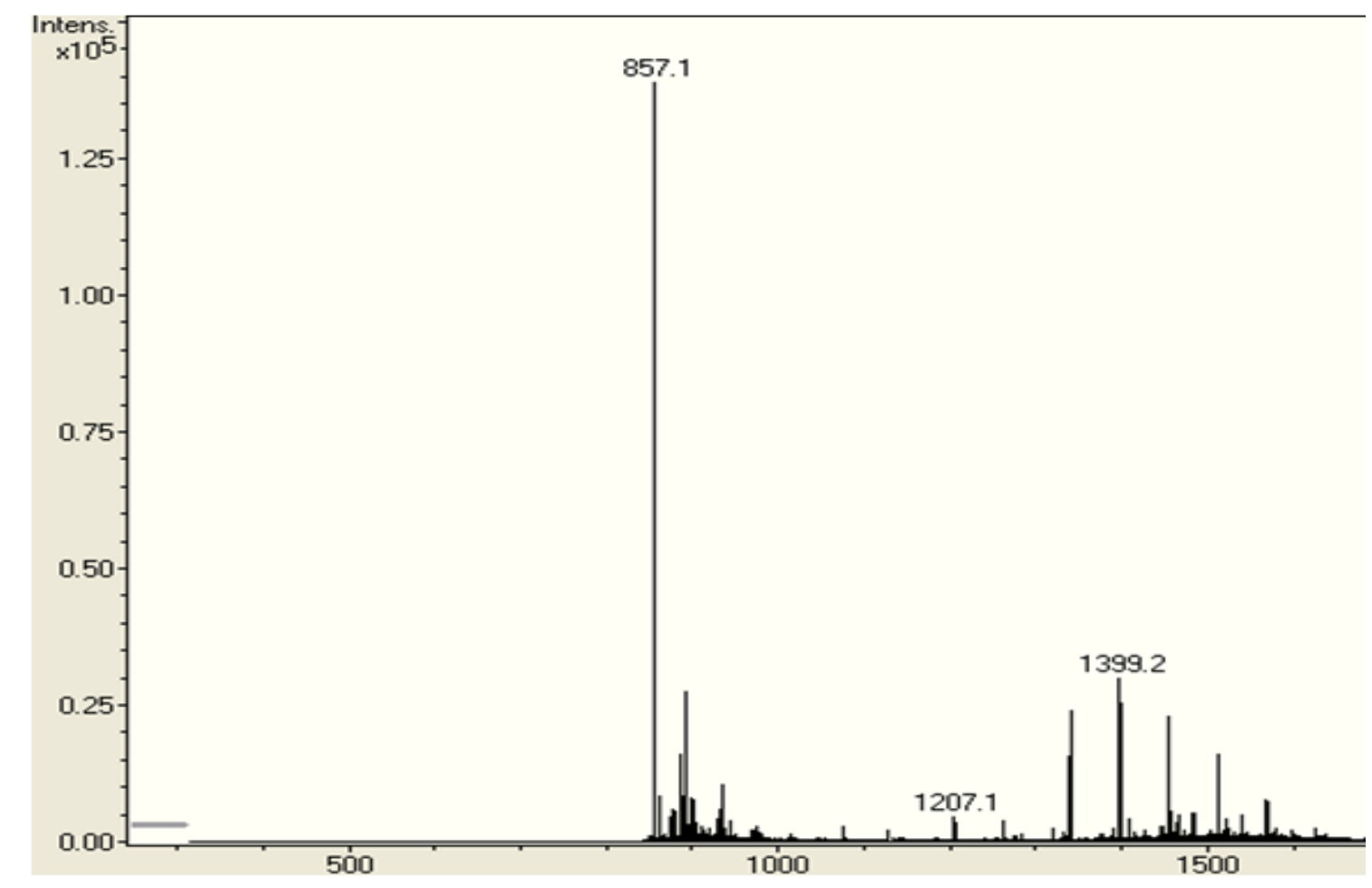

Spectrum 7: Mass spectrum of $\mathrm{C}(\mathrm{KAFKRK})_{3}$ Coum peptide.

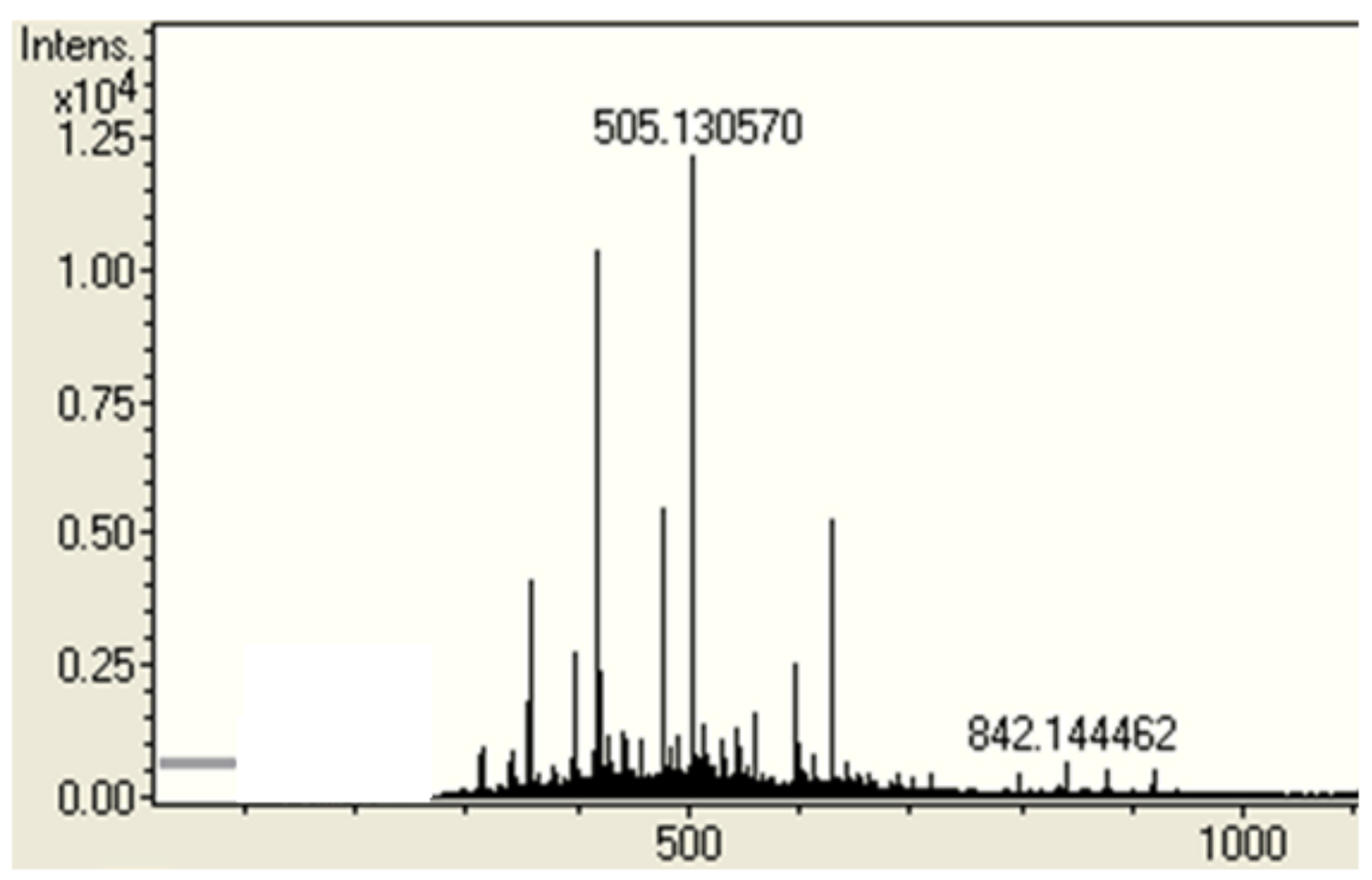

Spectrum 8: High resolution mass spectrum of $\mathrm{C}(\mathrm{KAFKRK})_{3}$ peptide. 


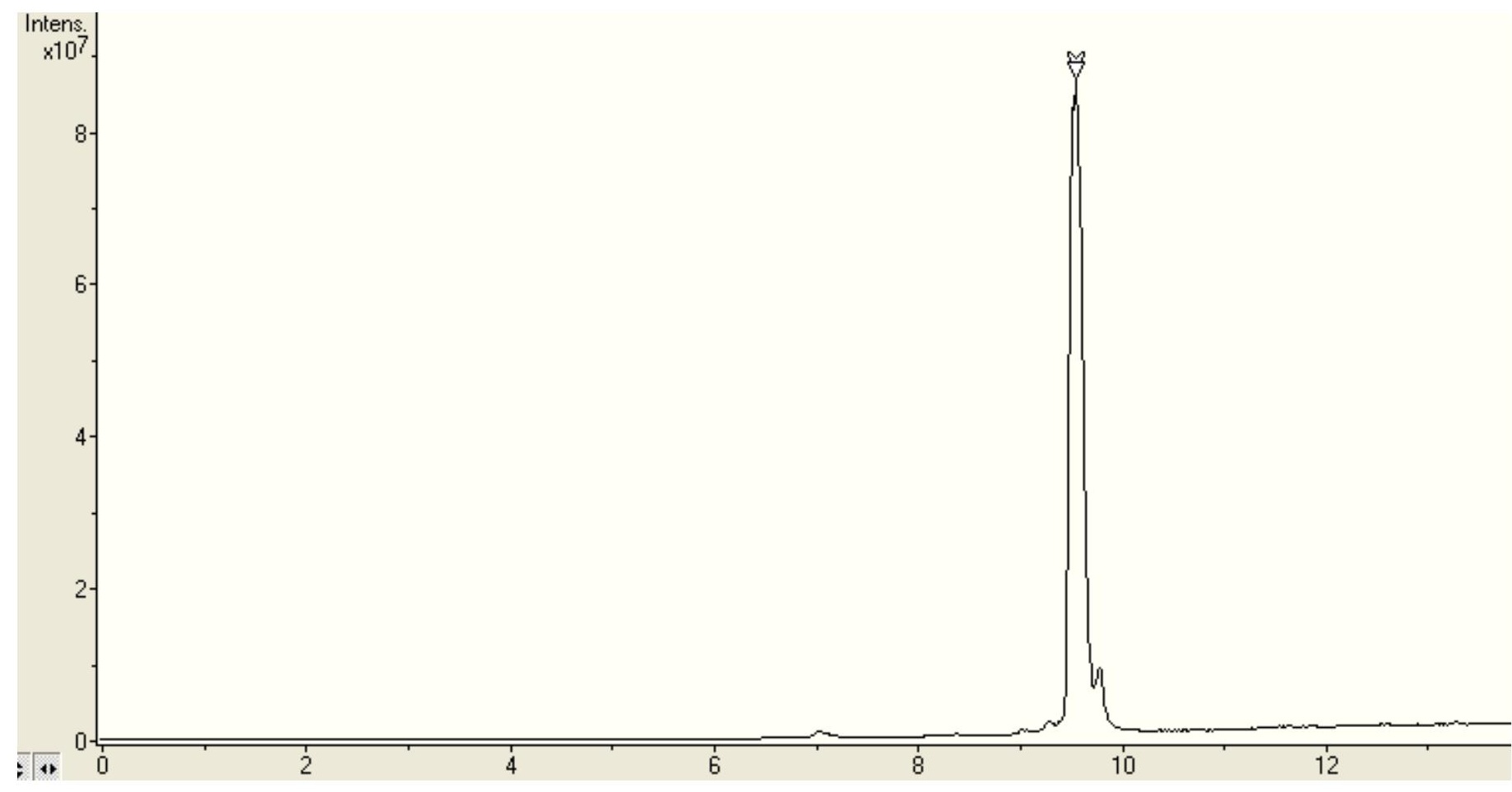

Chromatograph 3: LC-MS chromatograph of the peptide CGAFKGGCoum.

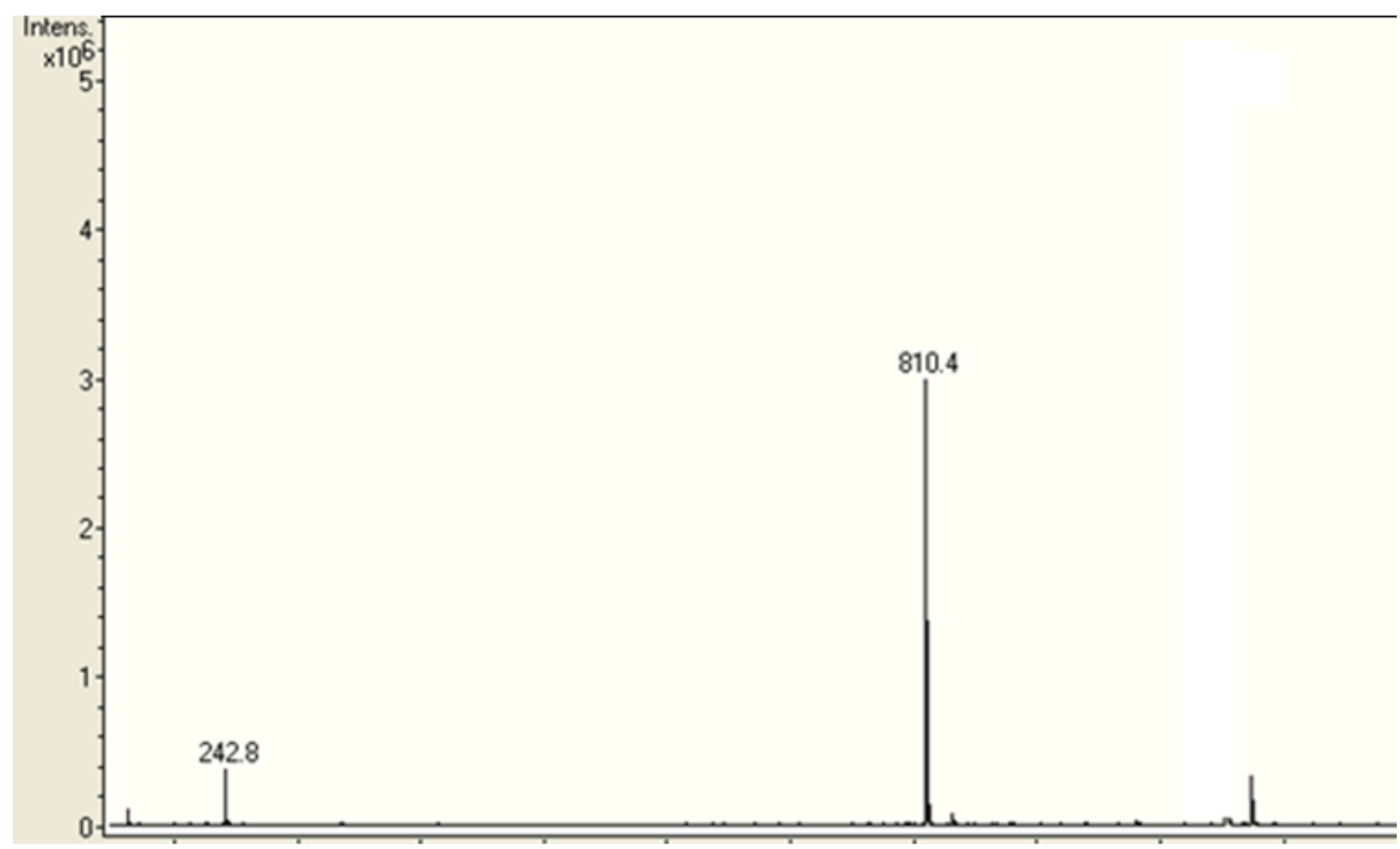

Spectrum 9: Mass spectrum of the peptide CGAFKGGCoum. 


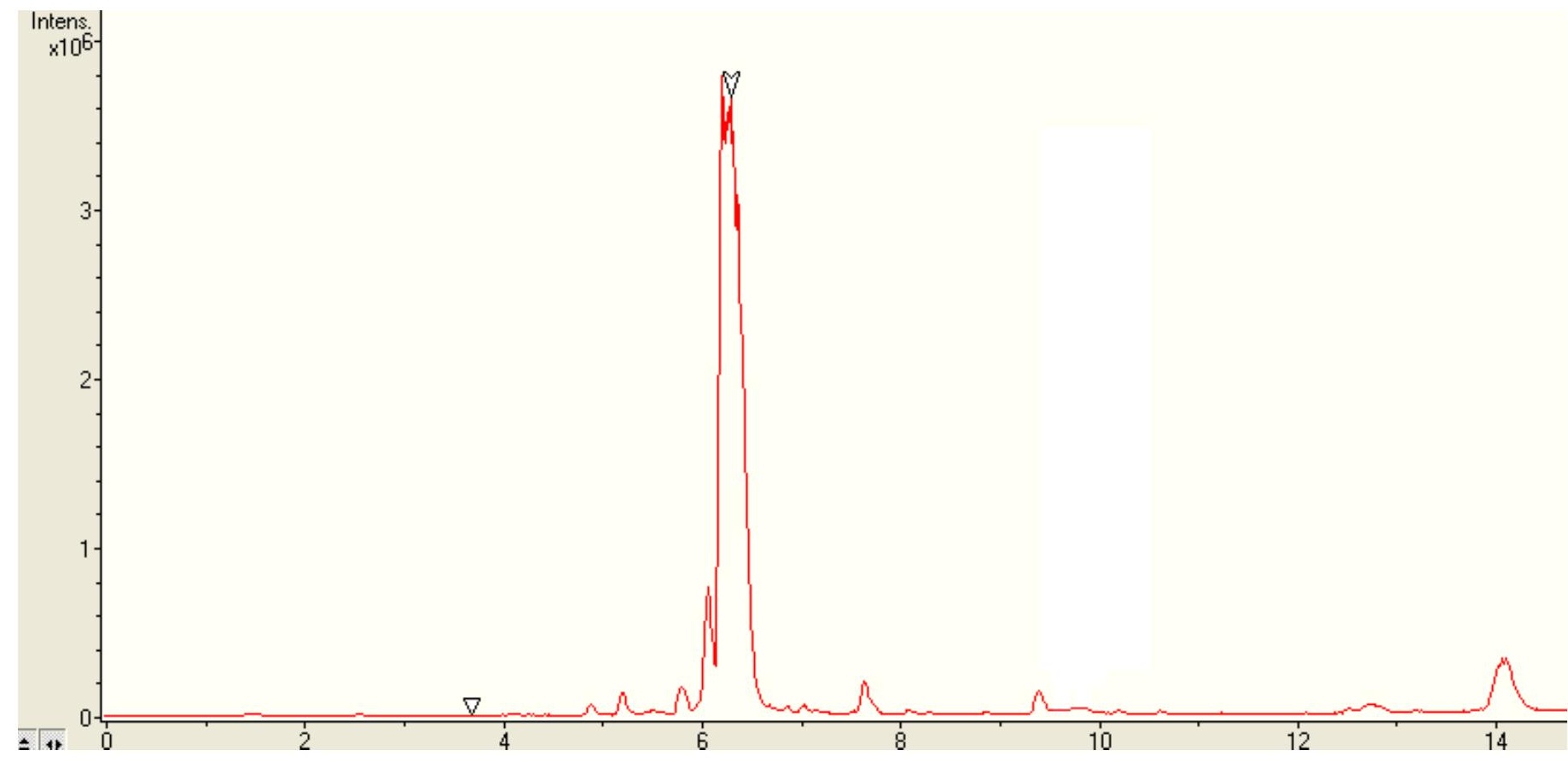

Chromatograph 4: LC-MS chromatograph of the peptide CKCoum.

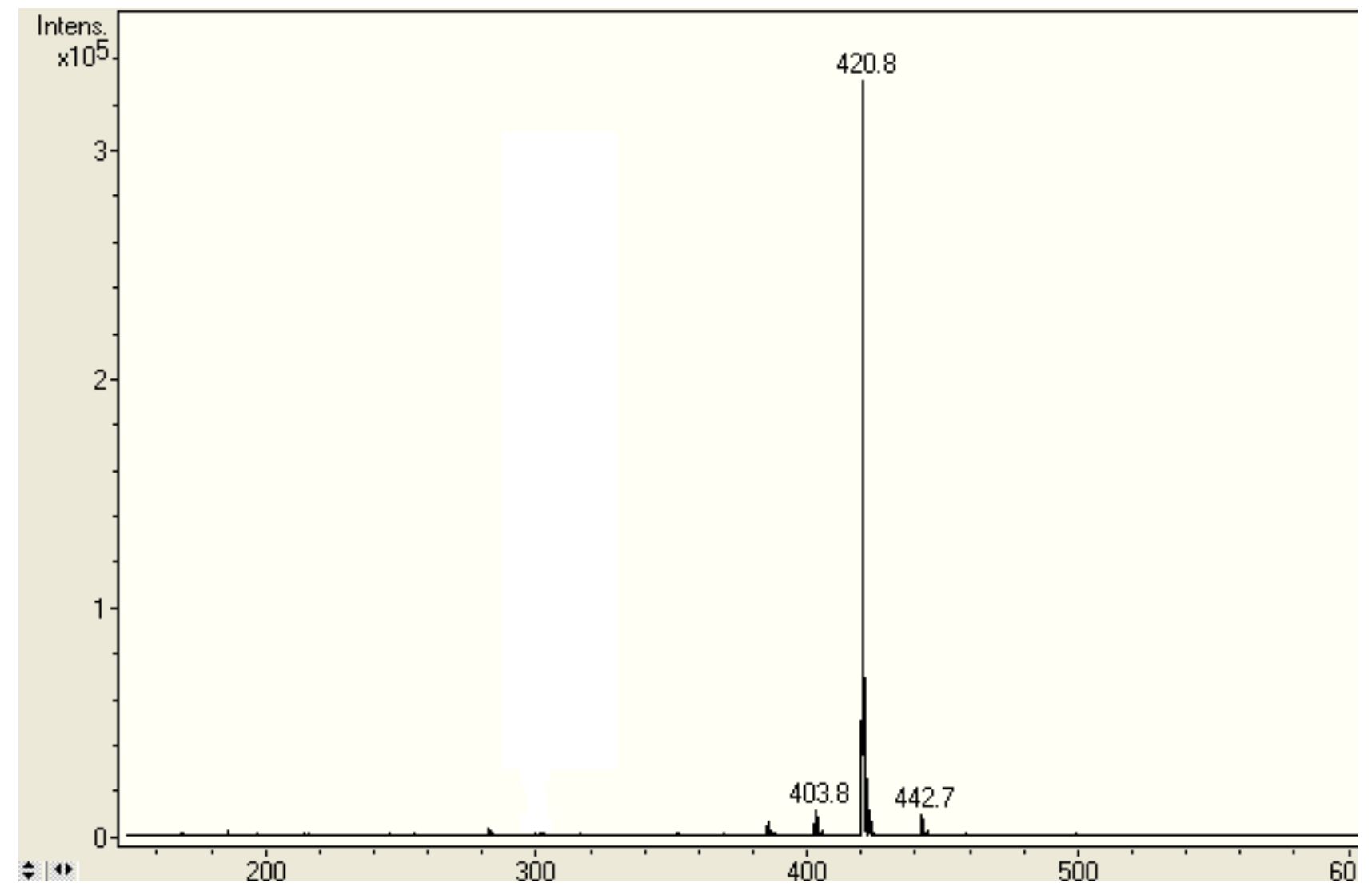

Spectrum 10: Mass spectrum of CKCoum peptide. 


\section{APPENDIX 2: CHARACTERIZATION OF GNPS}

IR and NMR spectroscopy were purely used as tool to verify the attachment of the ligands to the GNPs. IR and NMR characterization of the neat capping ligands and peptides was thus performed to compare to the spectra of the capped GNPs. The ligand/peptide capped GNP spectra are thus presented alongside their corresponding free ligand/peptide for ease of comparison. 


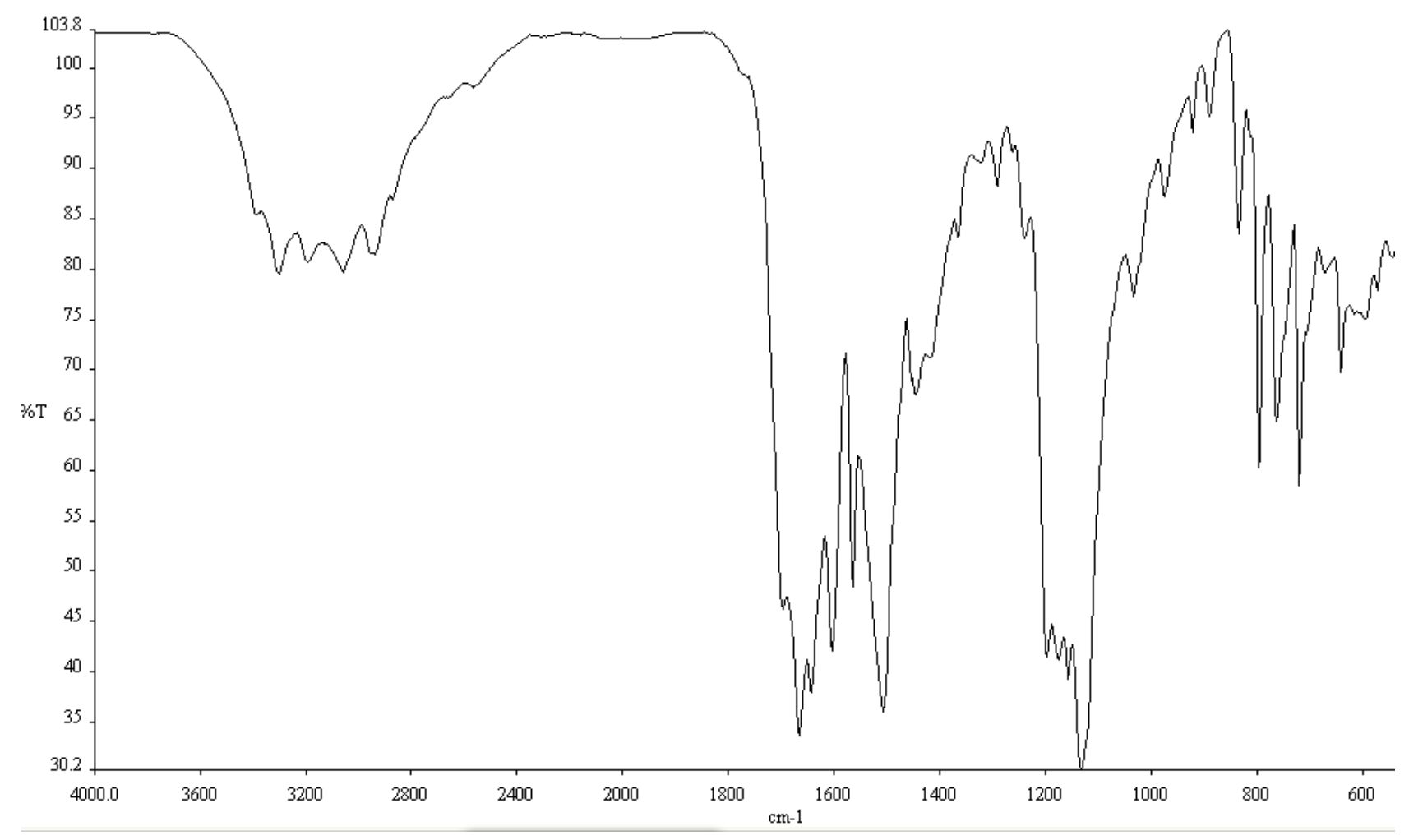

Spectrum 11: ATR-FTIR spectrum of CKCoum.

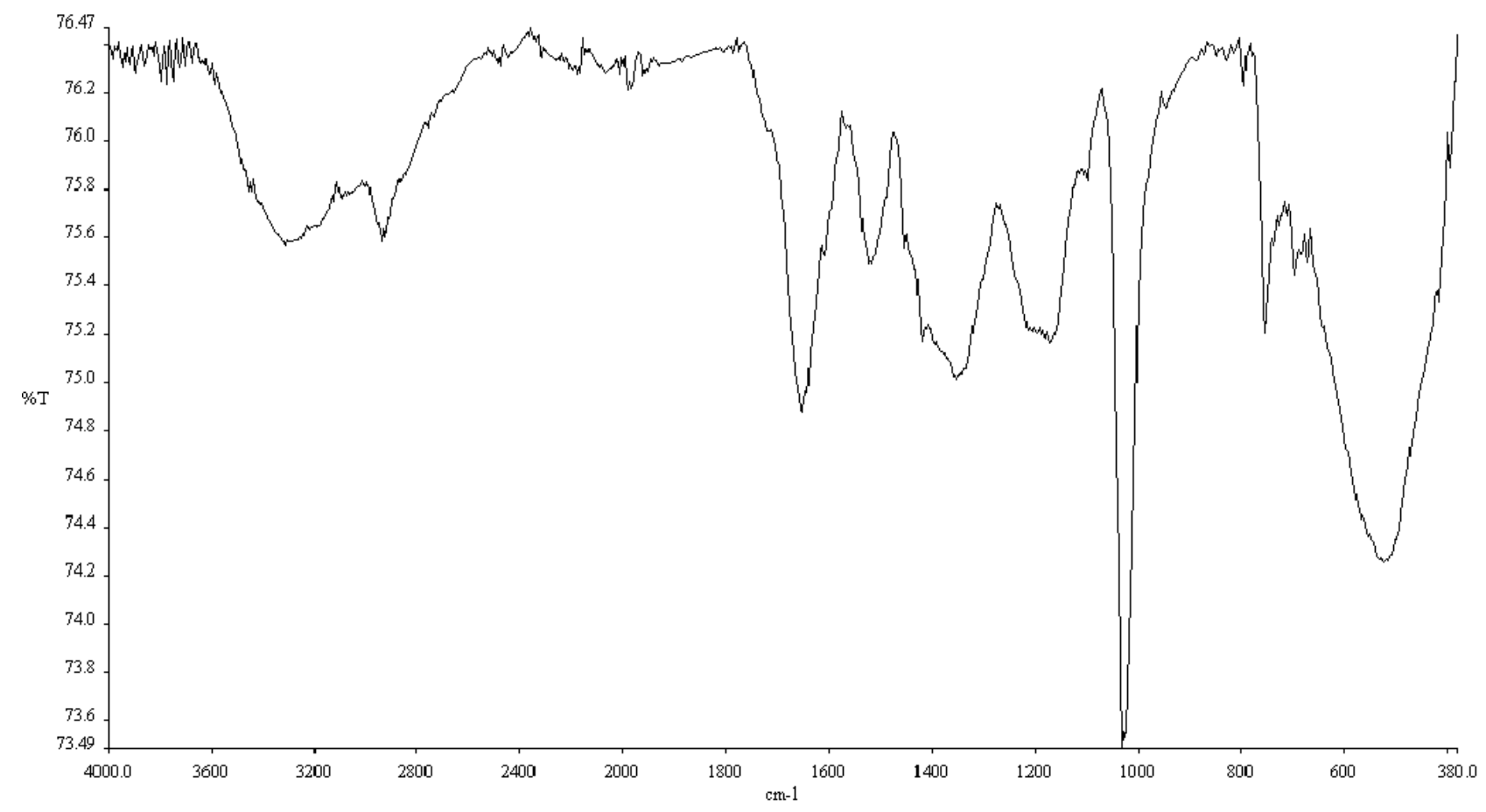

Spectrum 12: ATR-FTIR spectrum of Au-CKCoum. 


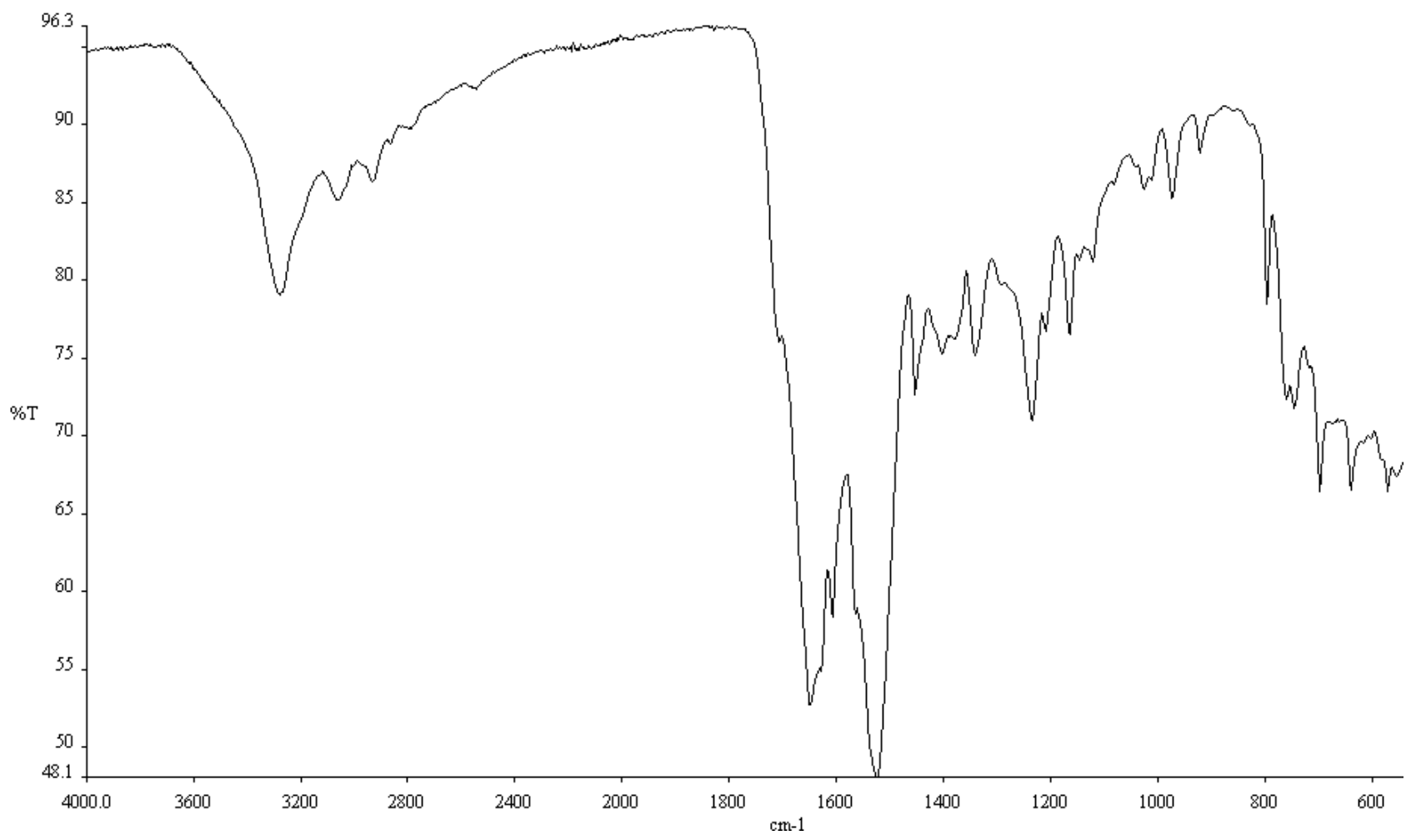

Spectrum 13: ATR-FTIR spectrum of CGAFKGGCoum.

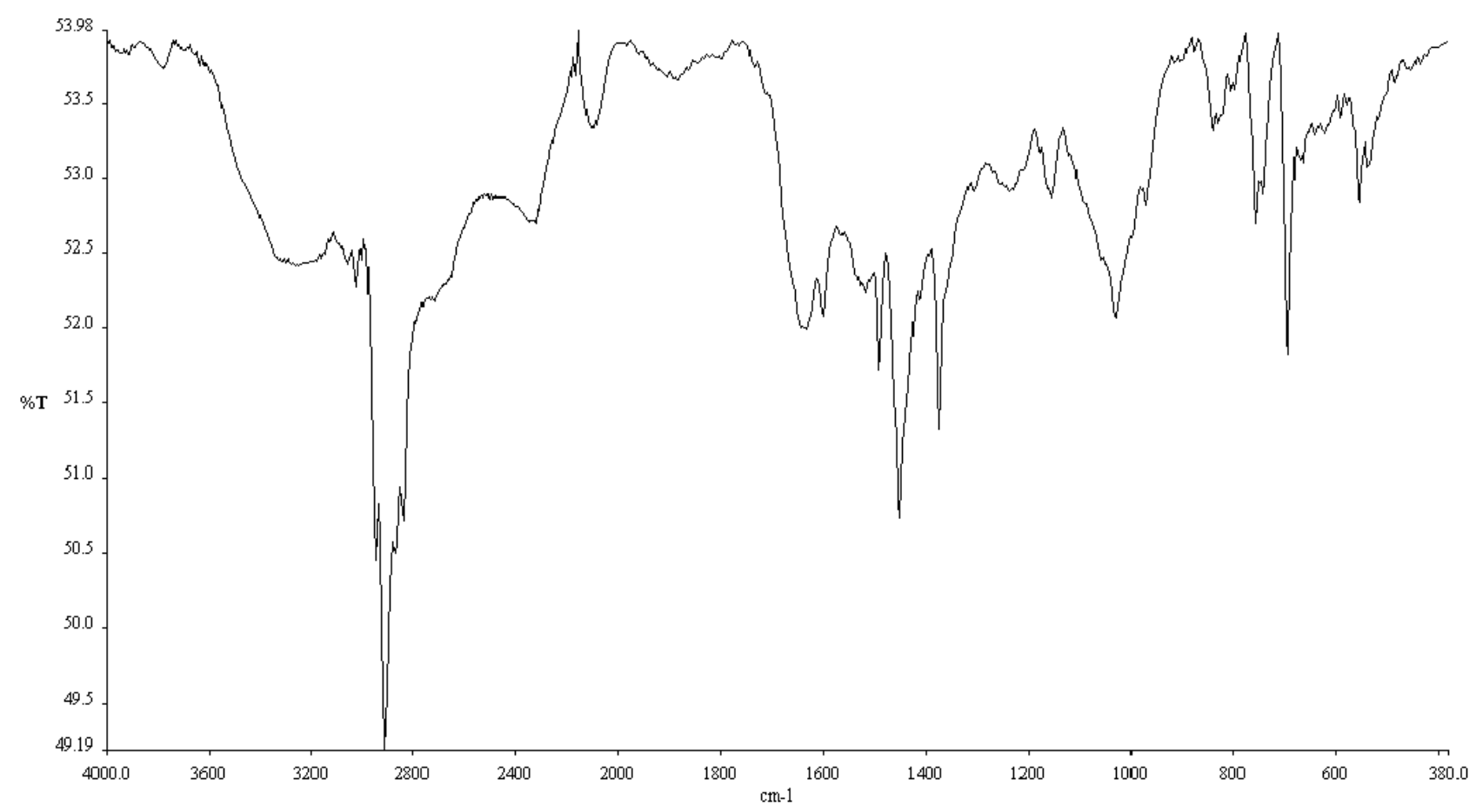

Spectrum 14: ATR-FTIR spectrum of Au-CGAFKGGCoum. 


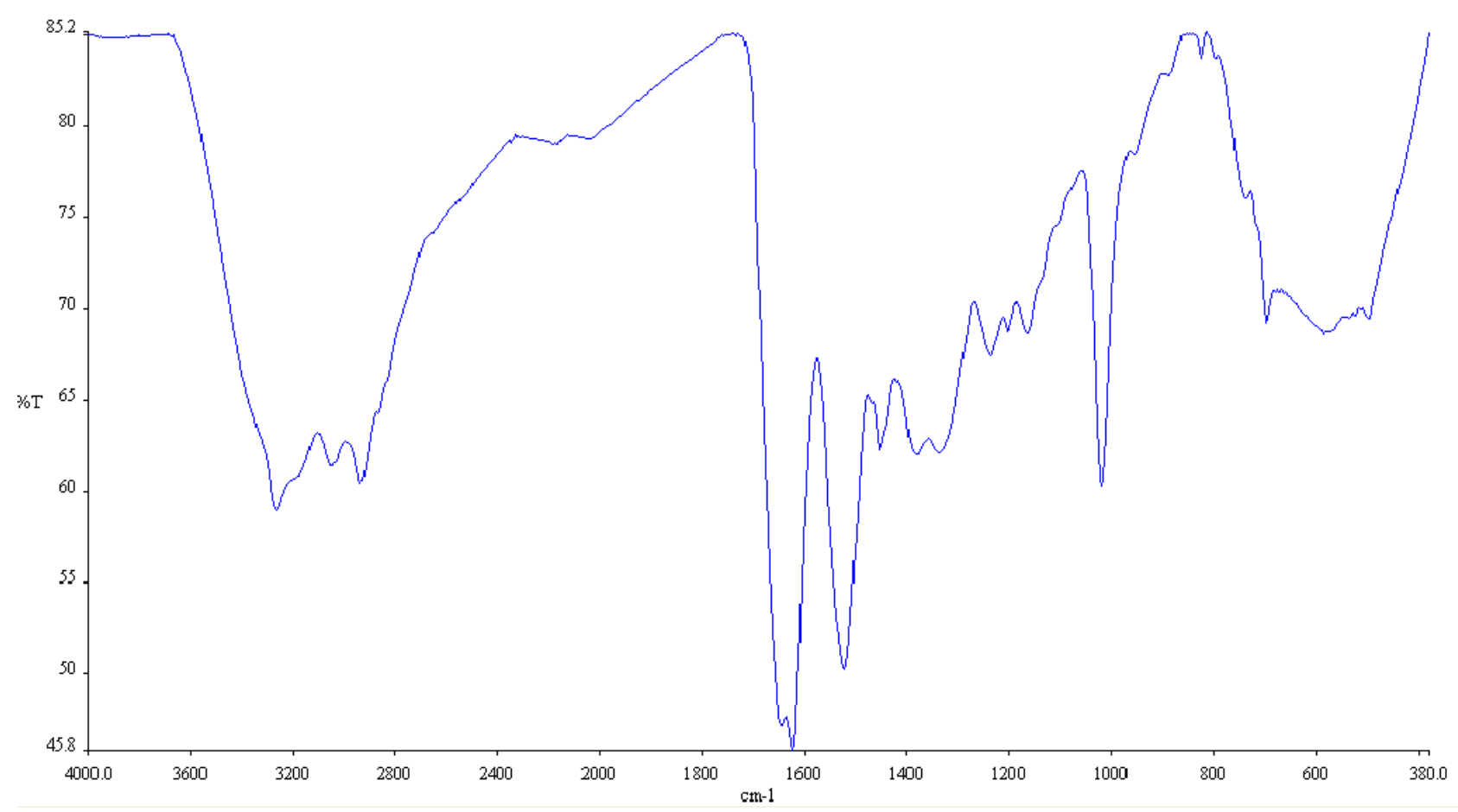

Spectrum 15: ATR-FTIR spectrum of C(KAFKRK $)_{3}$ Coum.

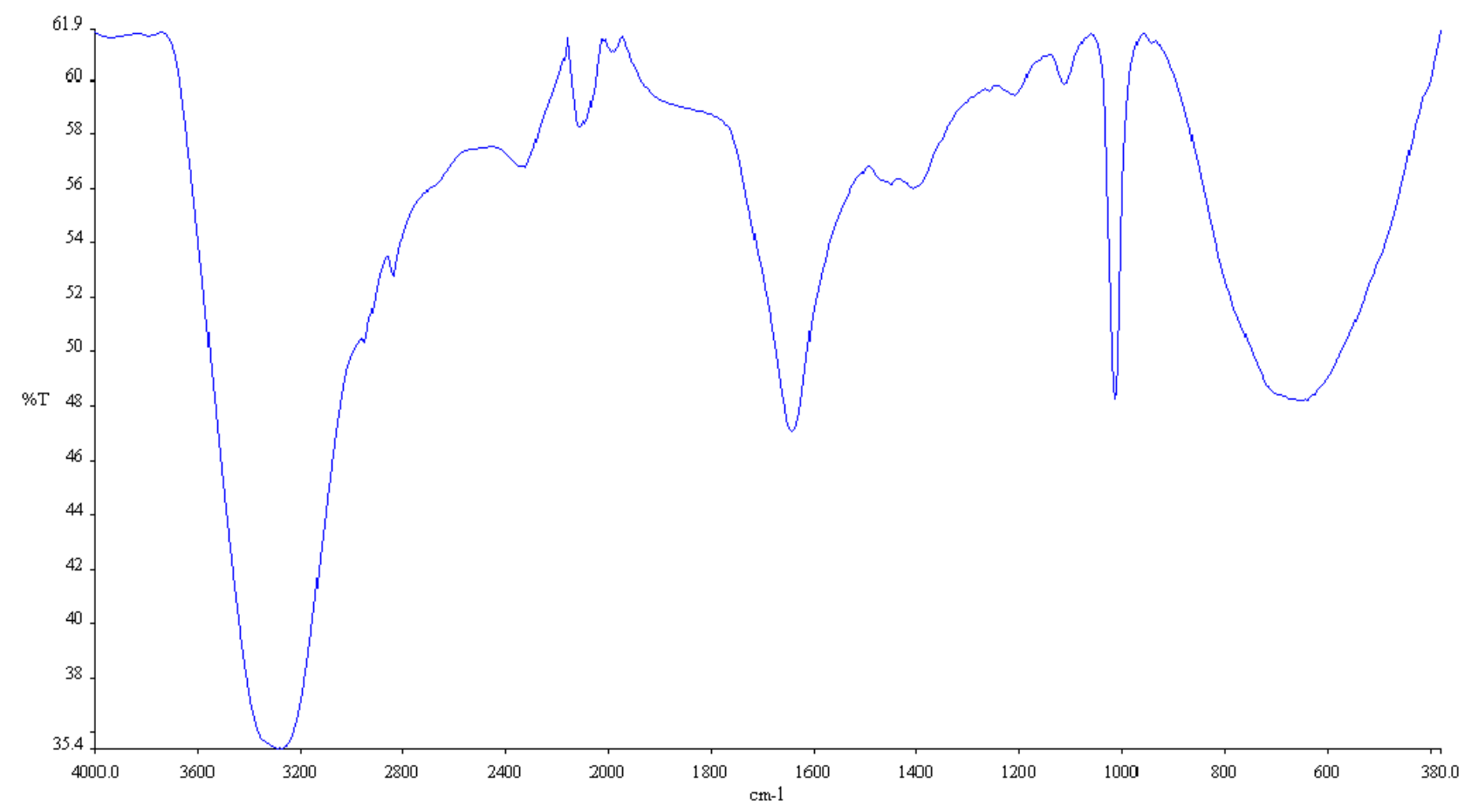

Spectrum 16: ATR-FTIR spectrum of $\mathrm{Au}-\mathrm{C}(\mathrm{KAFKRK}){ }_{3} \mathrm{Coum}$. 


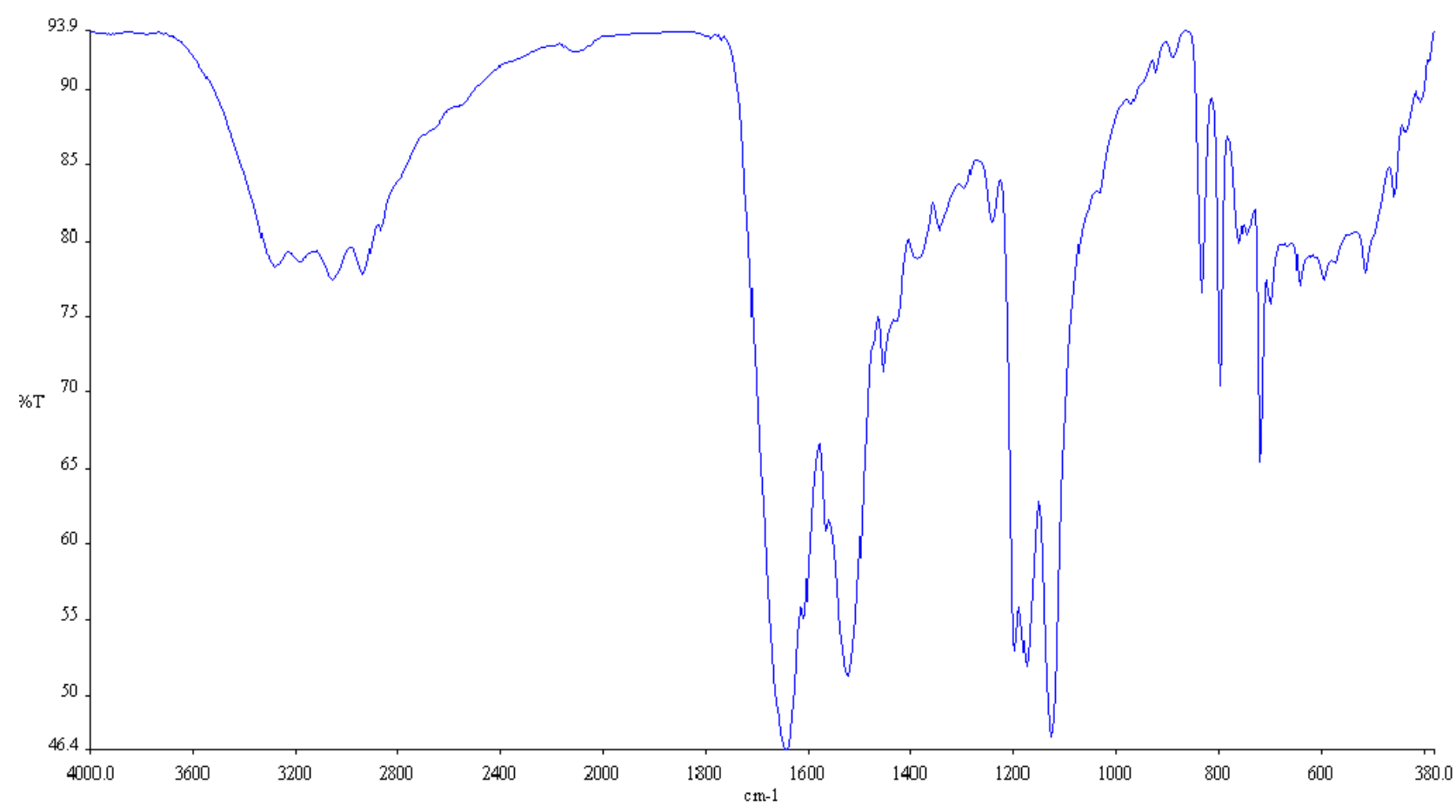

Spectrum 17: ATR-FTIR spectrum of CKAFKRKCoum.

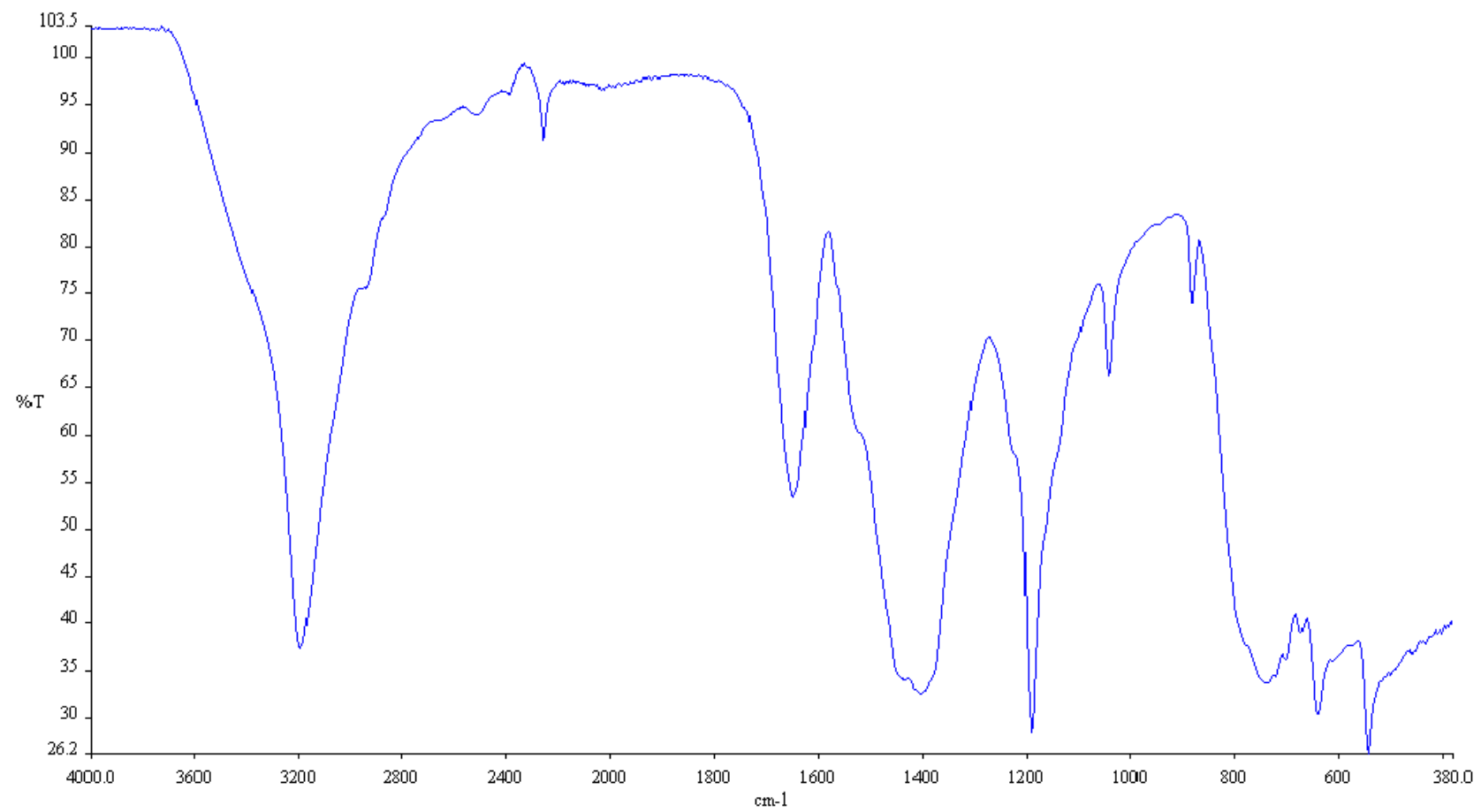

Spectrum 18: ATR-FTIR spectrum of Au-CKAFKRKCoum. 


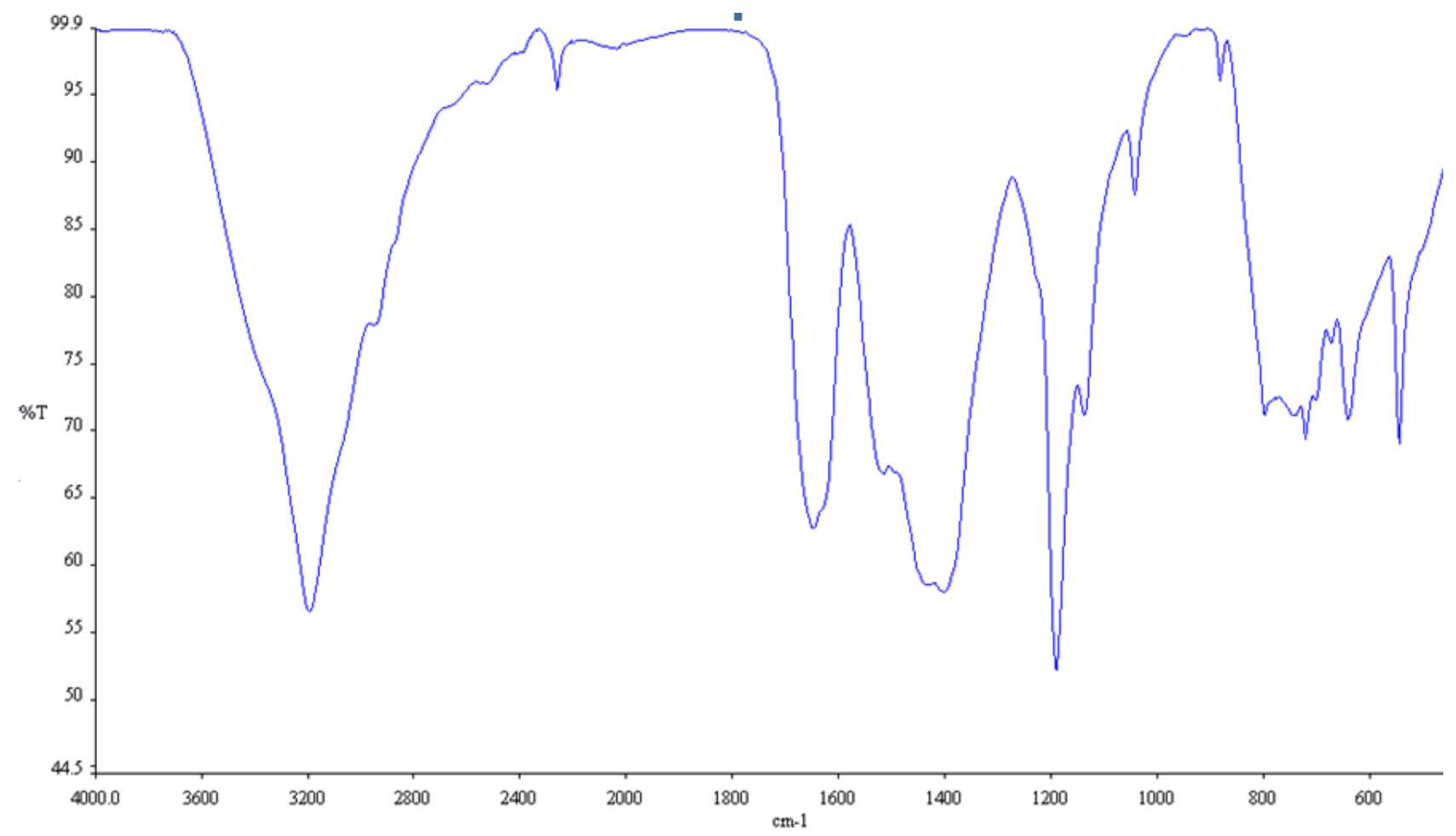

Spectrum 19: ATR-FTIR spectrum of Au-10\% CKAFKRKCoum:40\% CKAFKRK:50\% $\mathrm{C}(\mathrm{KAFKRK})_{3}$. 


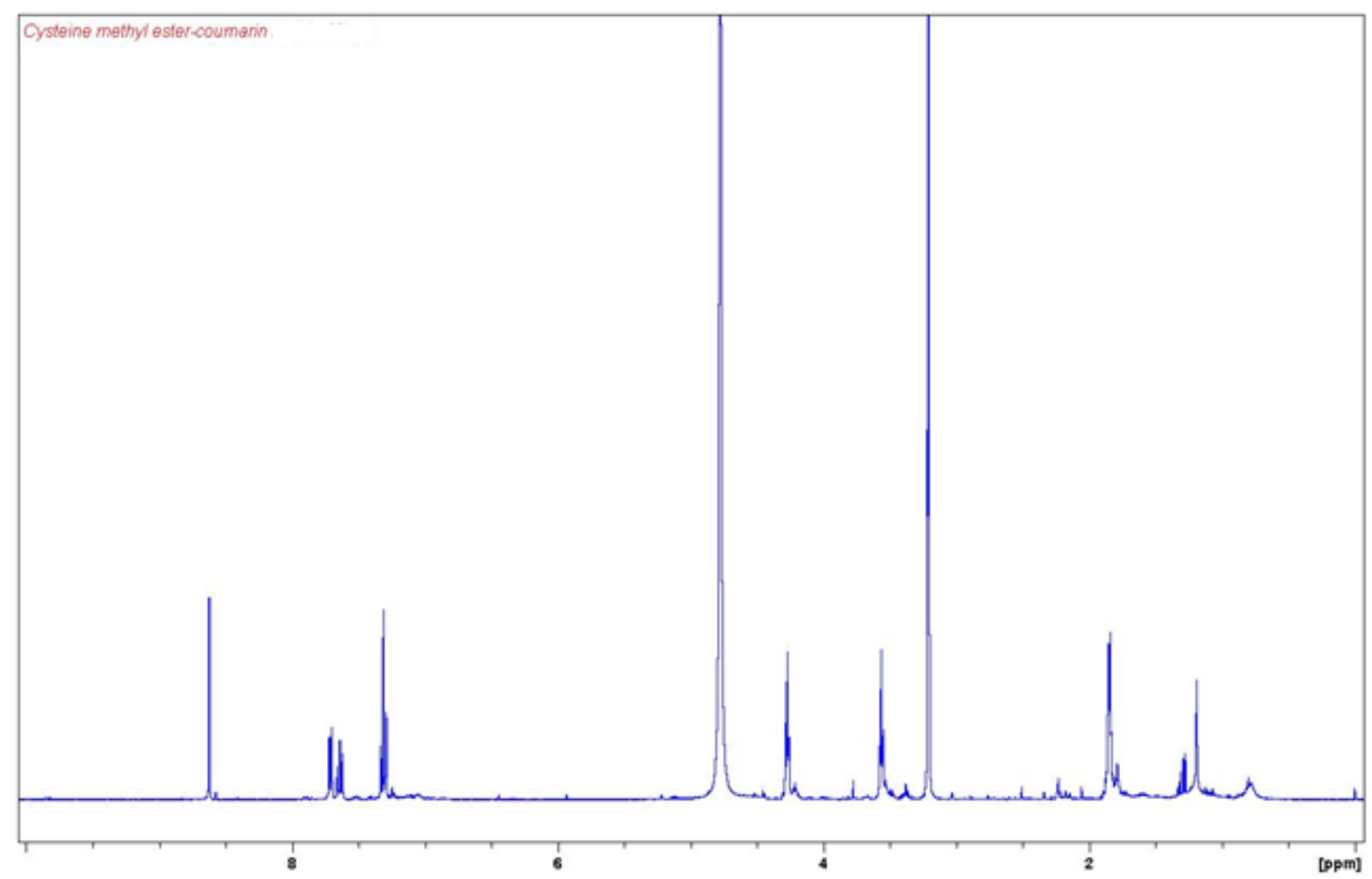

Spectrum 20: ${ }^{1} \mathrm{H}$ NMR spectrum of CMECoum in $\mathrm{CD}_{3} \mathrm{OD}$.

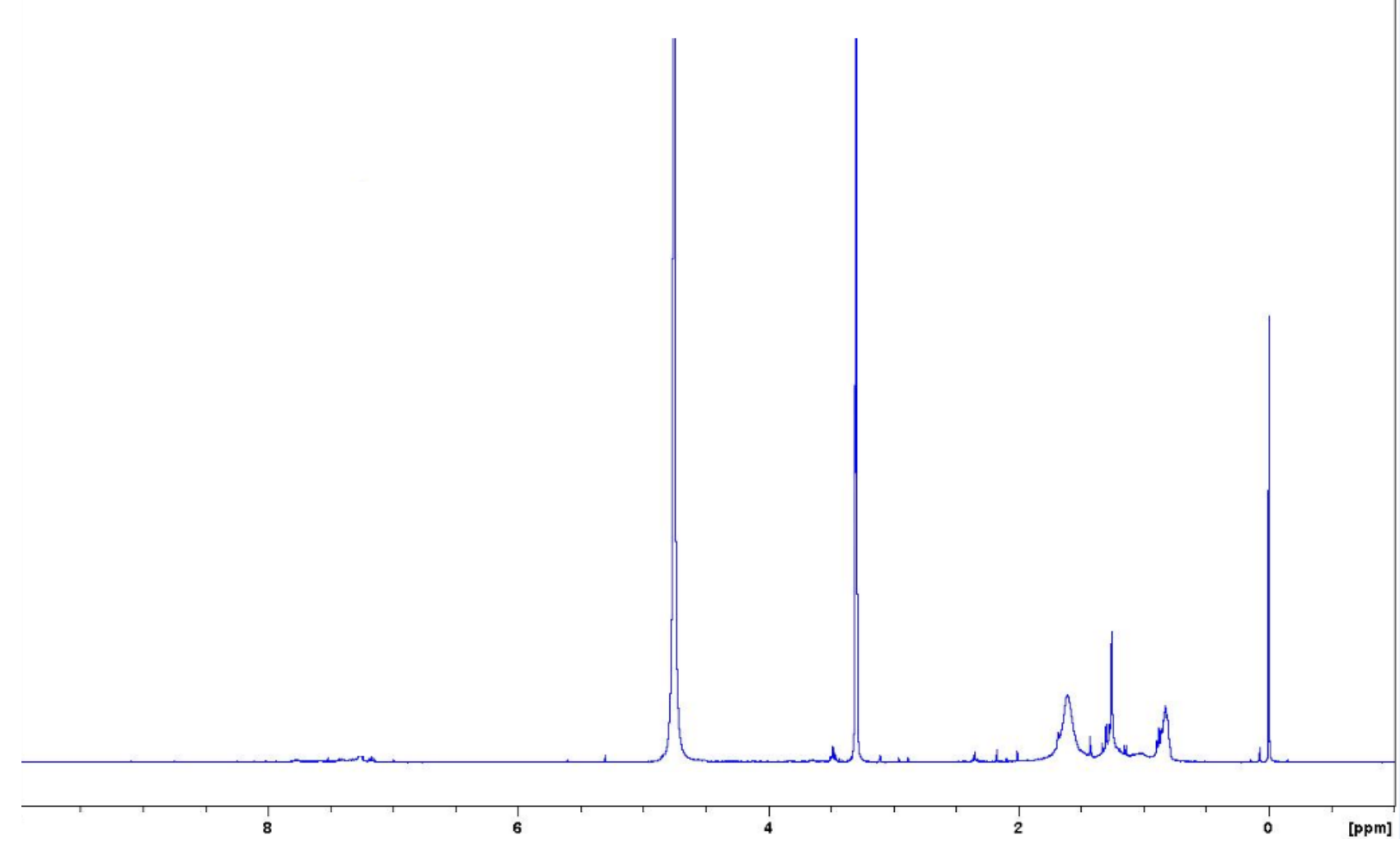

Spectrum 21: ${ }^{1} \mathrm{H}$ NMR spectrum of Au-CMECoum in $\mathrm{CD}_{3} \mathrm{OD}$. 


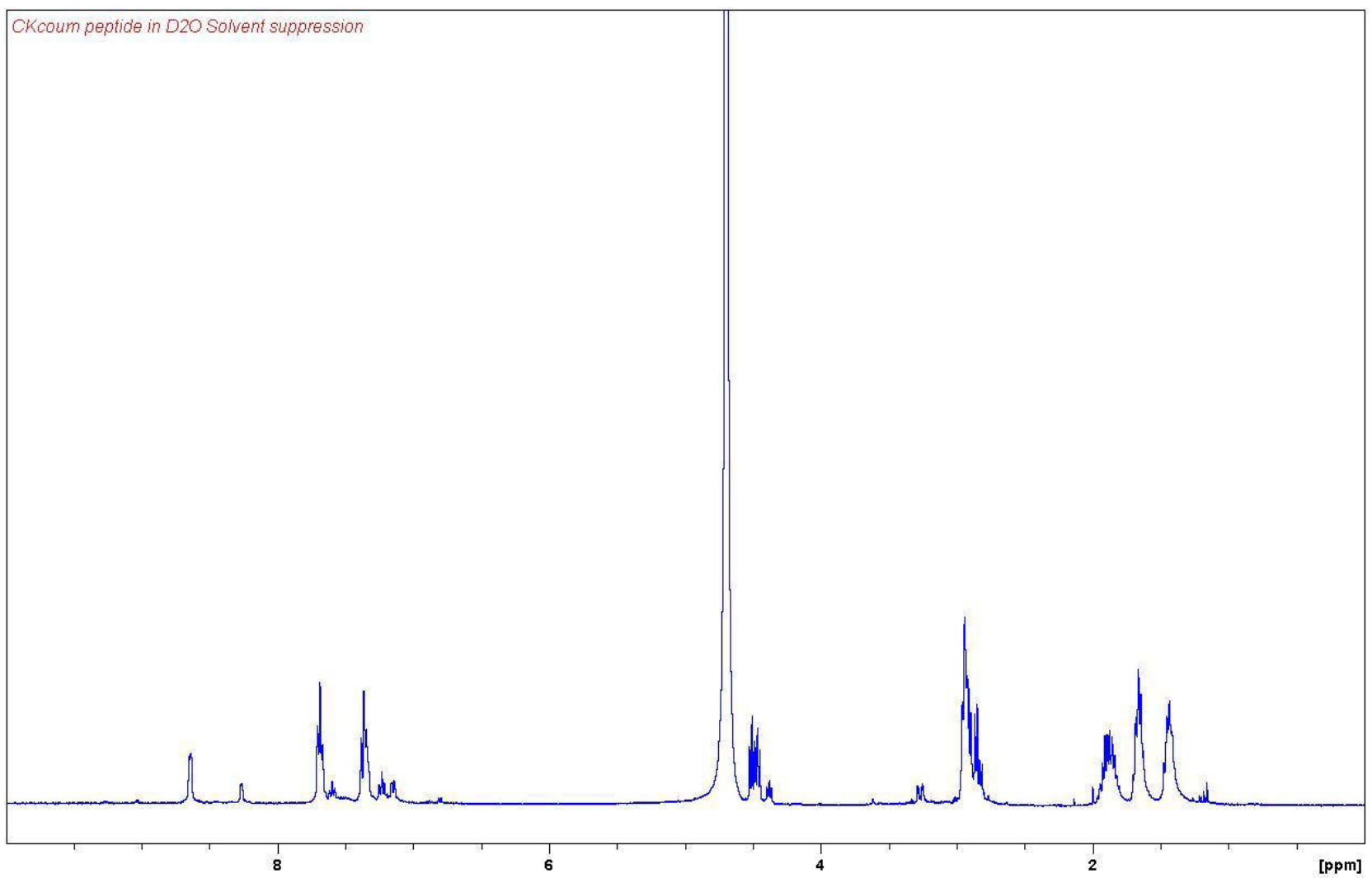

Spectrum 22: ${ }^{1} \mathrm{H}$ NMR spectrum of CKCoum in $\mathrm{D}_{2} \mathrm{O}$.

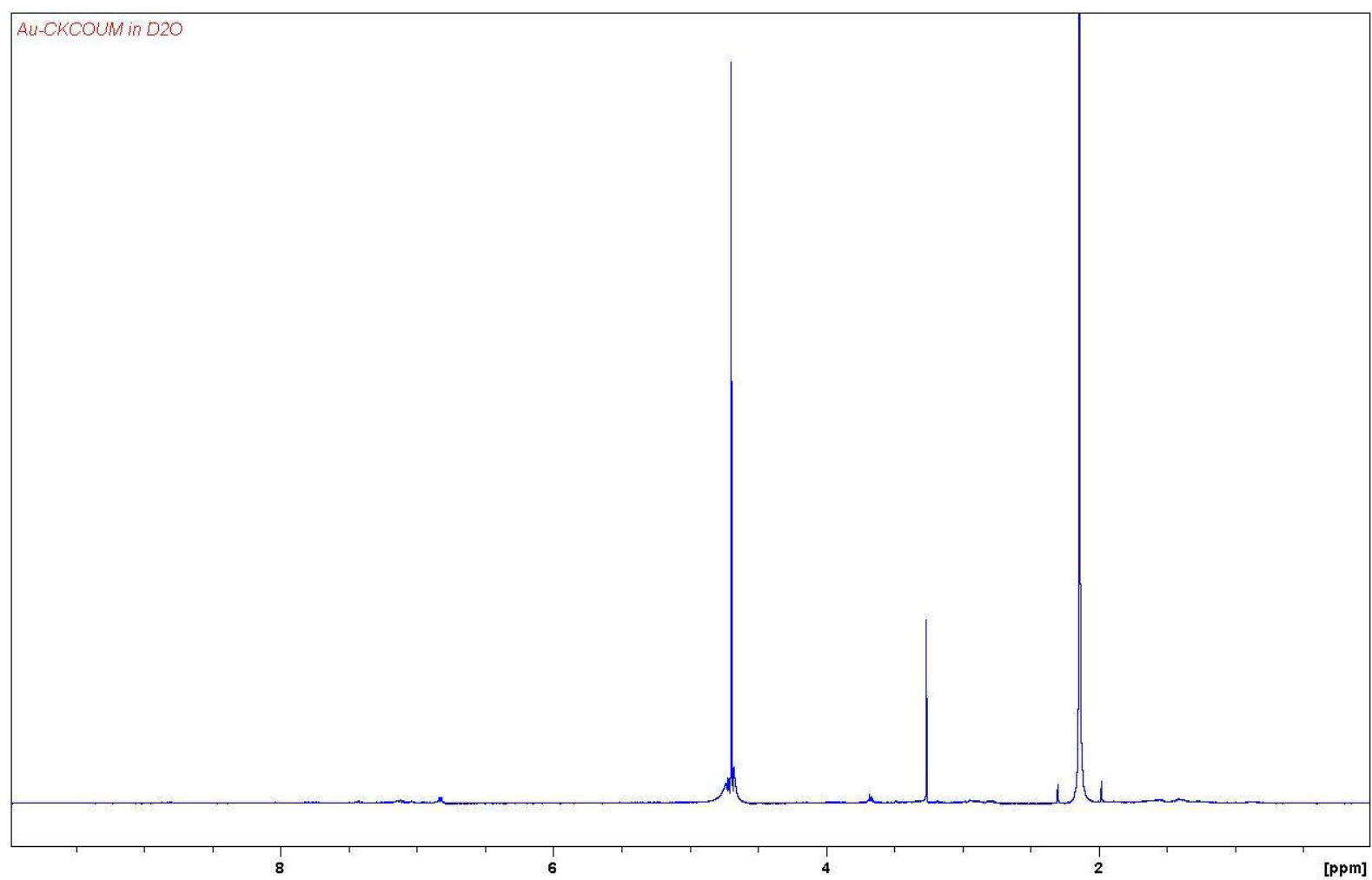

Spectrum 23: ${ }^{1} \mathrm{H}$ NMR spectrum of Au-CKCoum in $\mathrm{D}_{2} \mathrm{O}$. 


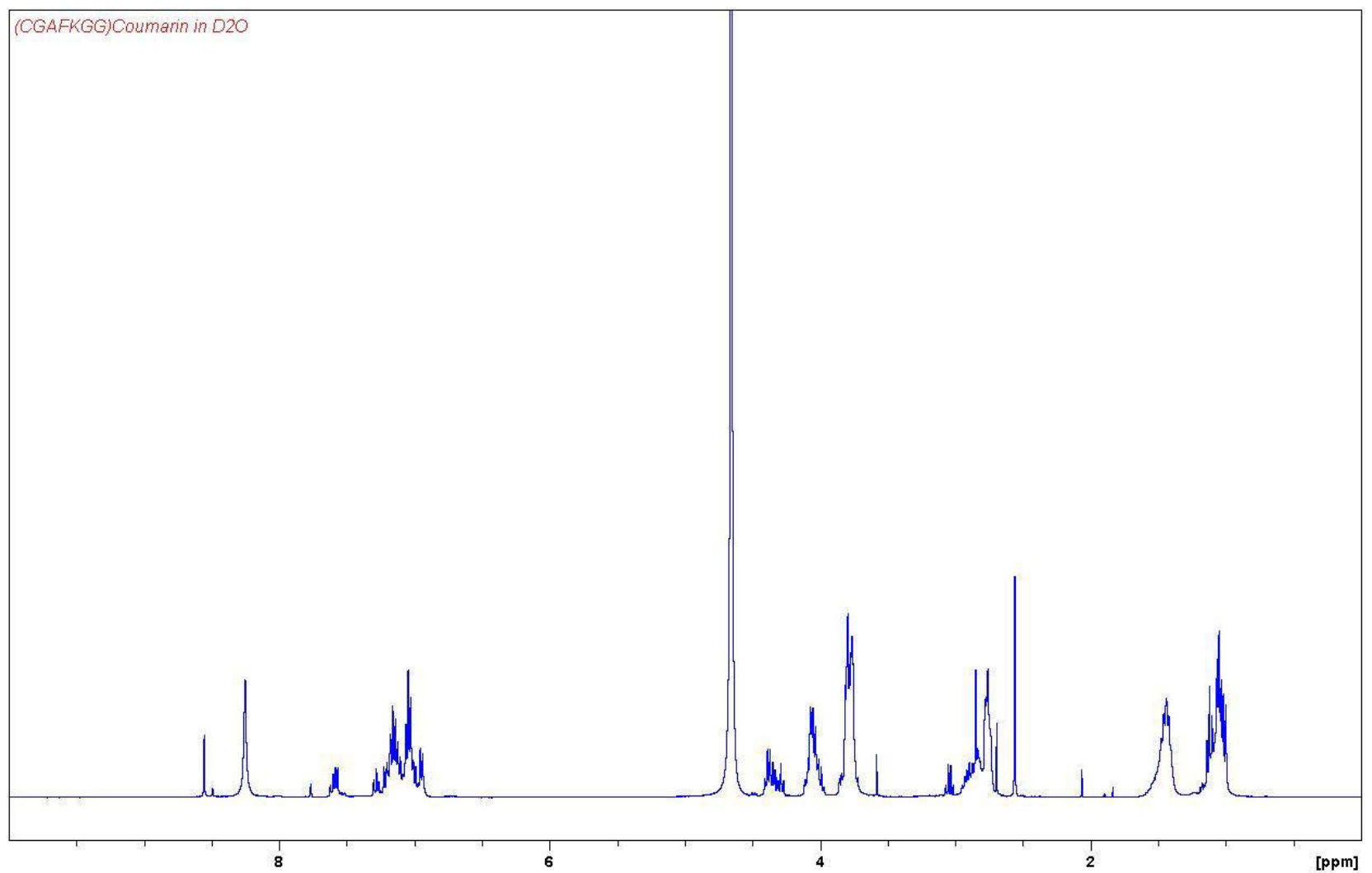

Spectrum 24: ${ }^{1} \mathrm{H}$ NMR spectrum of CGAFKGGCoum in $\mathrm{D}_{2} \mathrm{O}$.

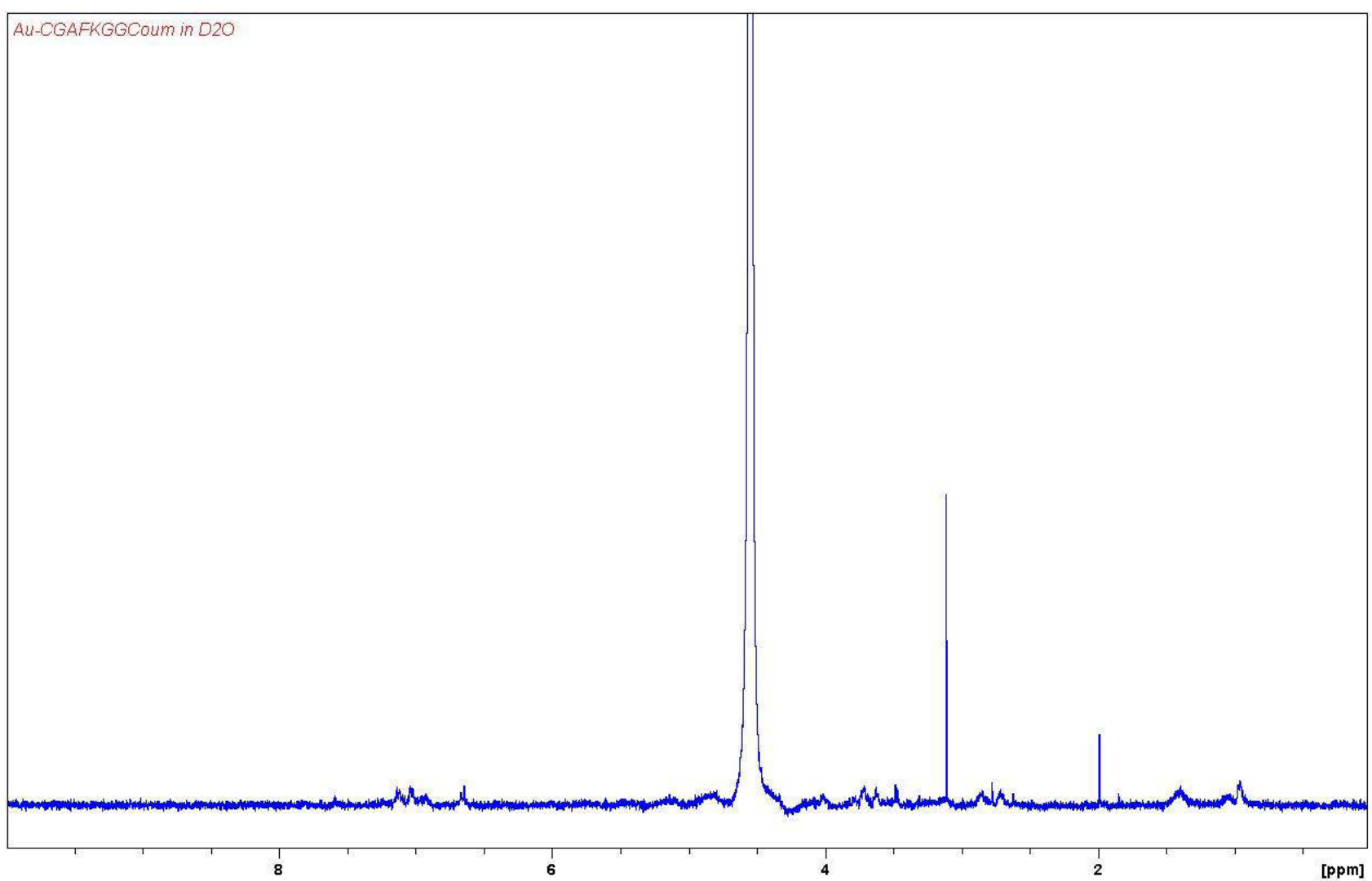

Spectrum 25: ${ }^{1} \mathrm{H}$ NMR spectrum of in Au-CGAFKGGCoum in $\mathrm{D}_{2} \mathrm{O}$. 


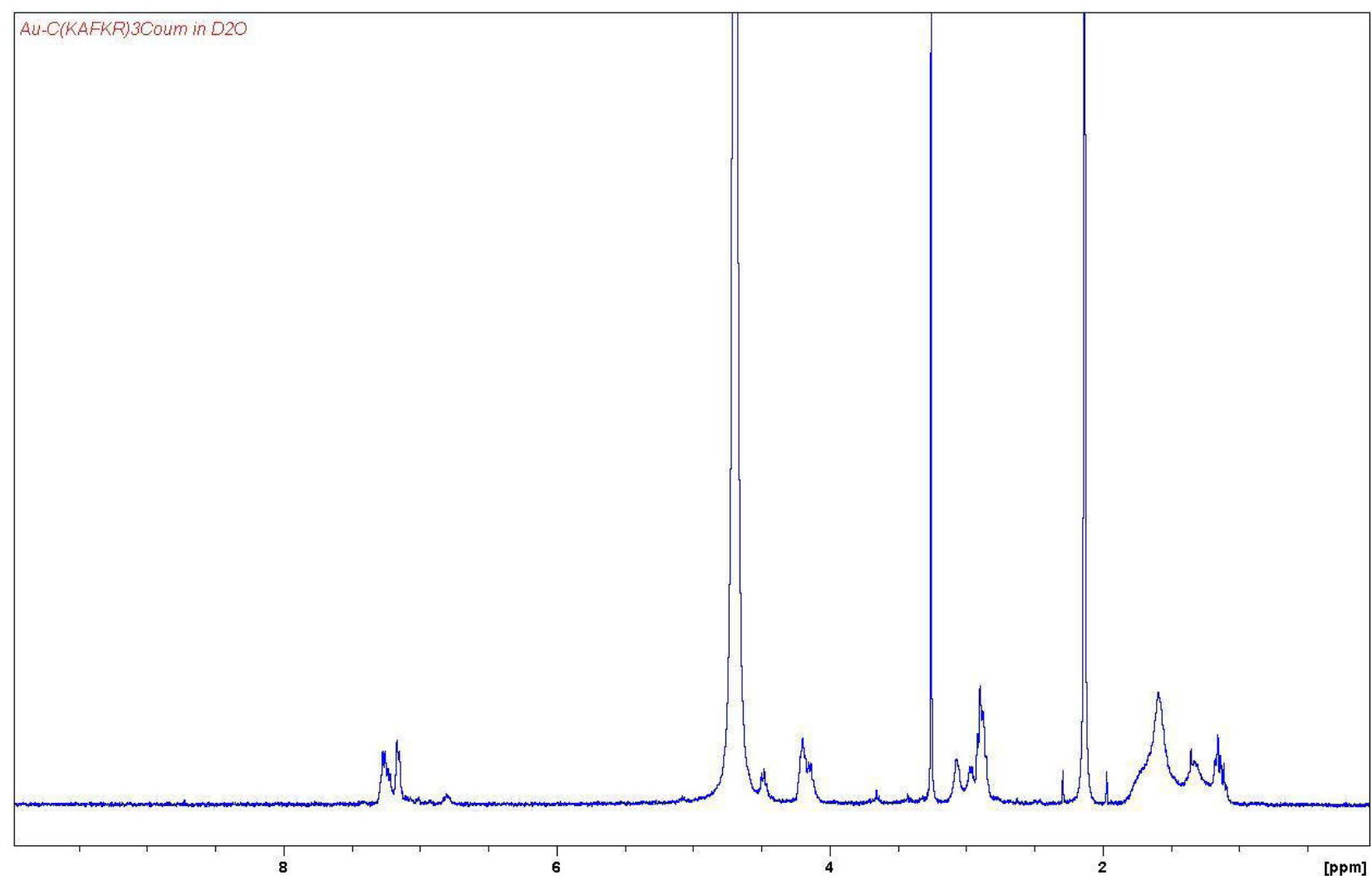

Spectrum 26: ${ }^{1} \mathrm{H}$ NMR spectrum of $\mathrm{C}(\mathrm{KAFKRK}){ }_{3}$ Coum in $\mathrm{D}_{2} \mathrm{O}$.

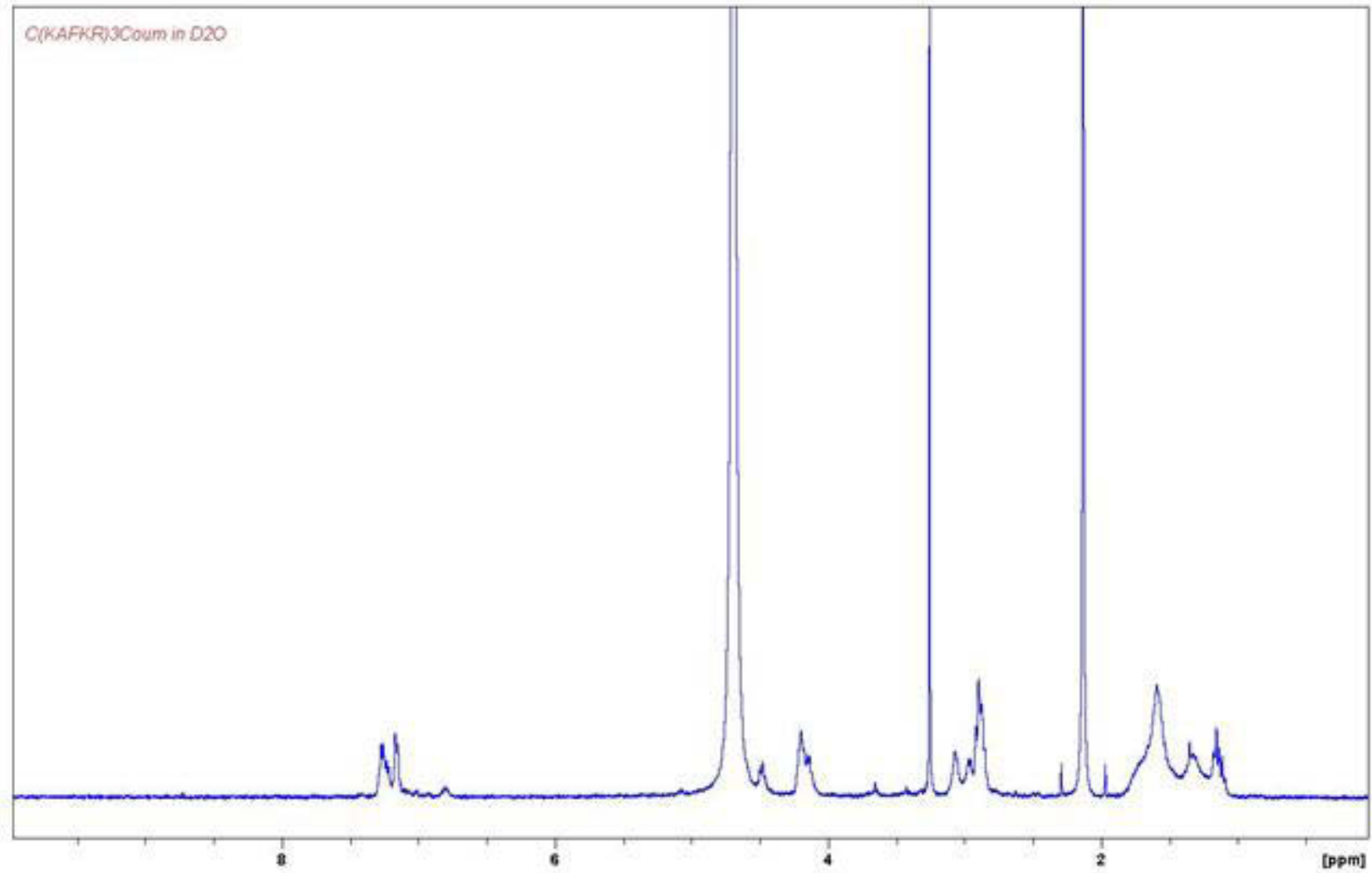

Spectrum 27: ${ }^{1} \mathrm{H}$ NMR spectrum of $\mathrm{Au}-\mathrm{C}(\mathrm{KAFKRK}){ }_{3}$ Coum in $\mathrm{D}_{2} \mathrm{O}$. 


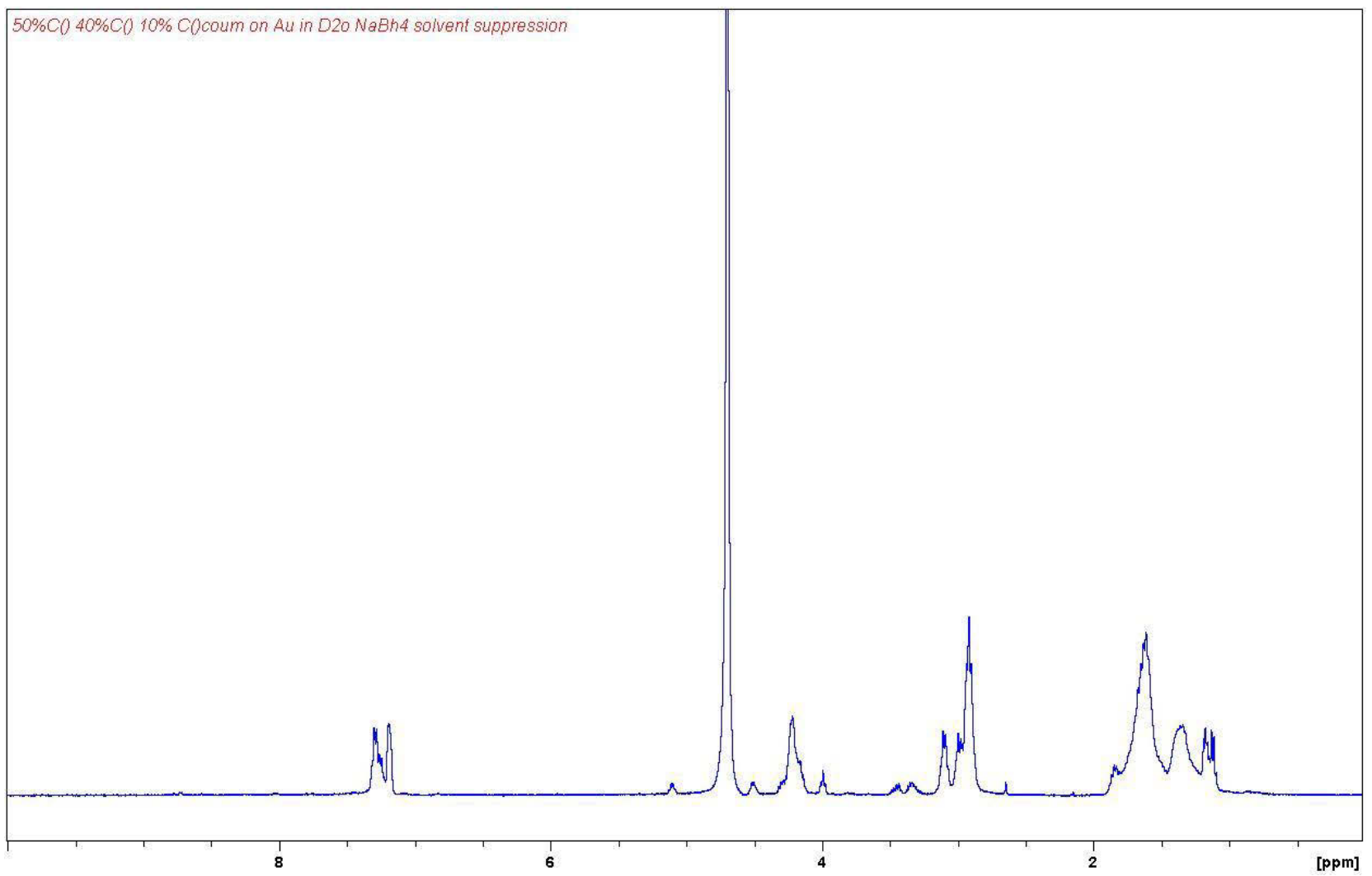

Spectrum 28: $\quad{ }^{1} \mathrm{H} \quad \mathrm{NMR}$ spectrum of Au-50\% C(KAFKRK) $)_{3}: 40 \% \quad$ CKAFKRK:10\% CKAFKRKCoum in $\mathrm{D}_{2} \mathrm{O}$. 
APPENDIX 3: ENZYMATIC DEGRADATION 


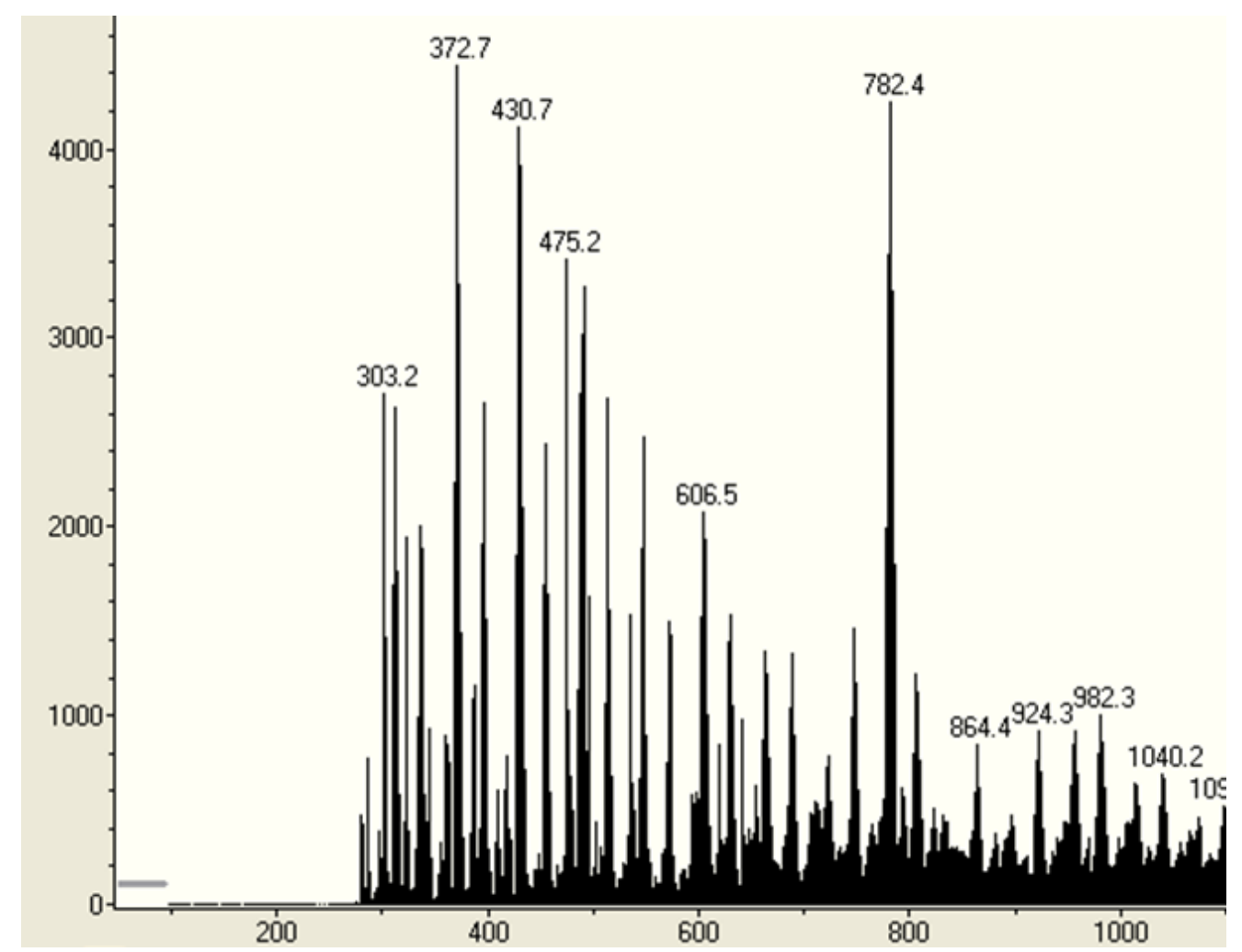

Spectrum 29: Mass spectrum of $\mathrm{Au}-\mathrm{C}(\mathrm{KAFKRK})_{3}$ in $\mathrm{PBS}$ prior to enzymatic degradation.

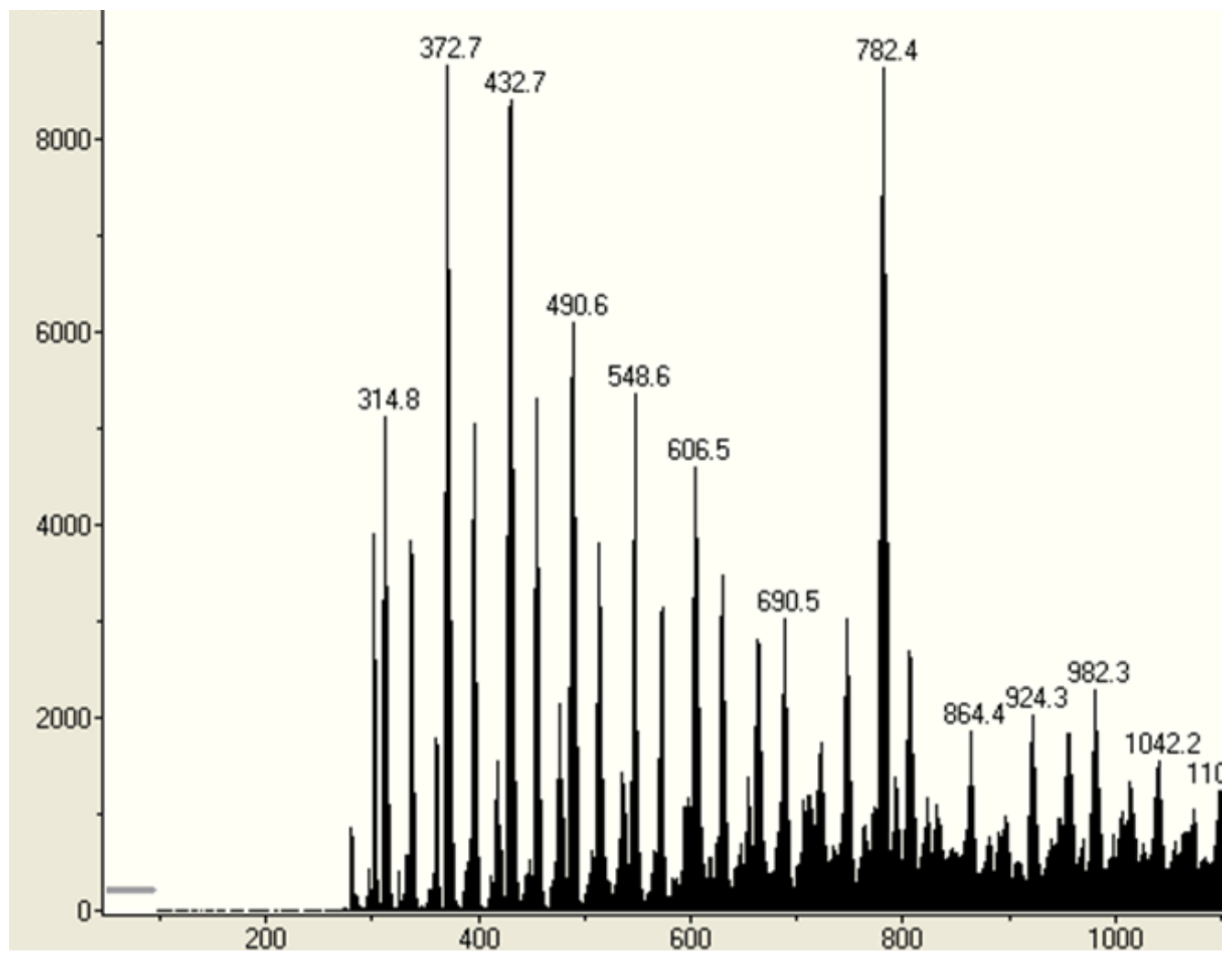

Spectrum 30: Mass spectrum of $\mathrm{Au}-\mathrm{C}(\mathrm{KAFKRK})_{3}$ in $\mathrm{PBS}$ after enzymatic degradation. 


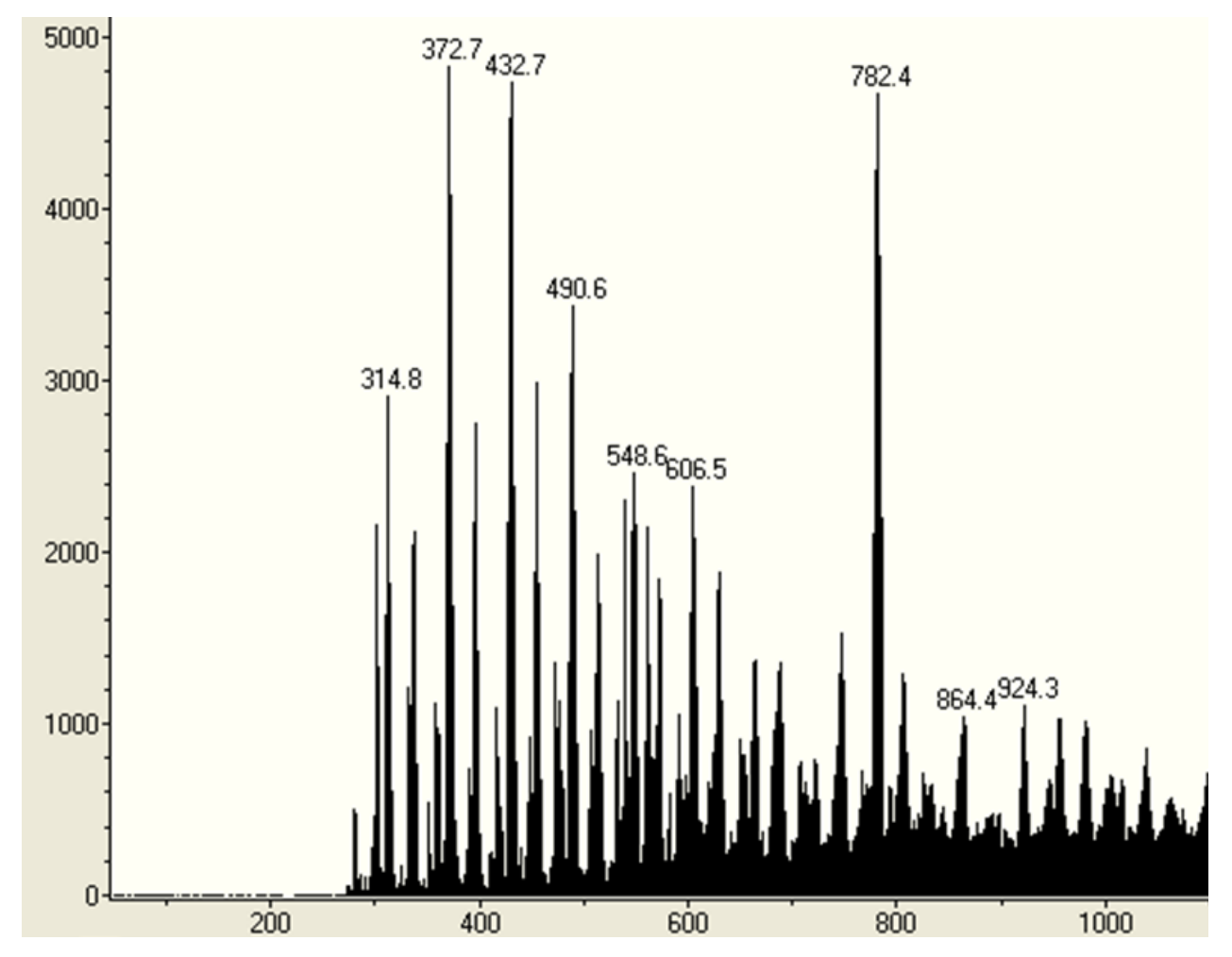

Spectrum 31: Mass spectrum of Au-CKAFKRKCoum:C(KAFKRK) 3 (50:50) in PBS prior to enzymatic degradation.

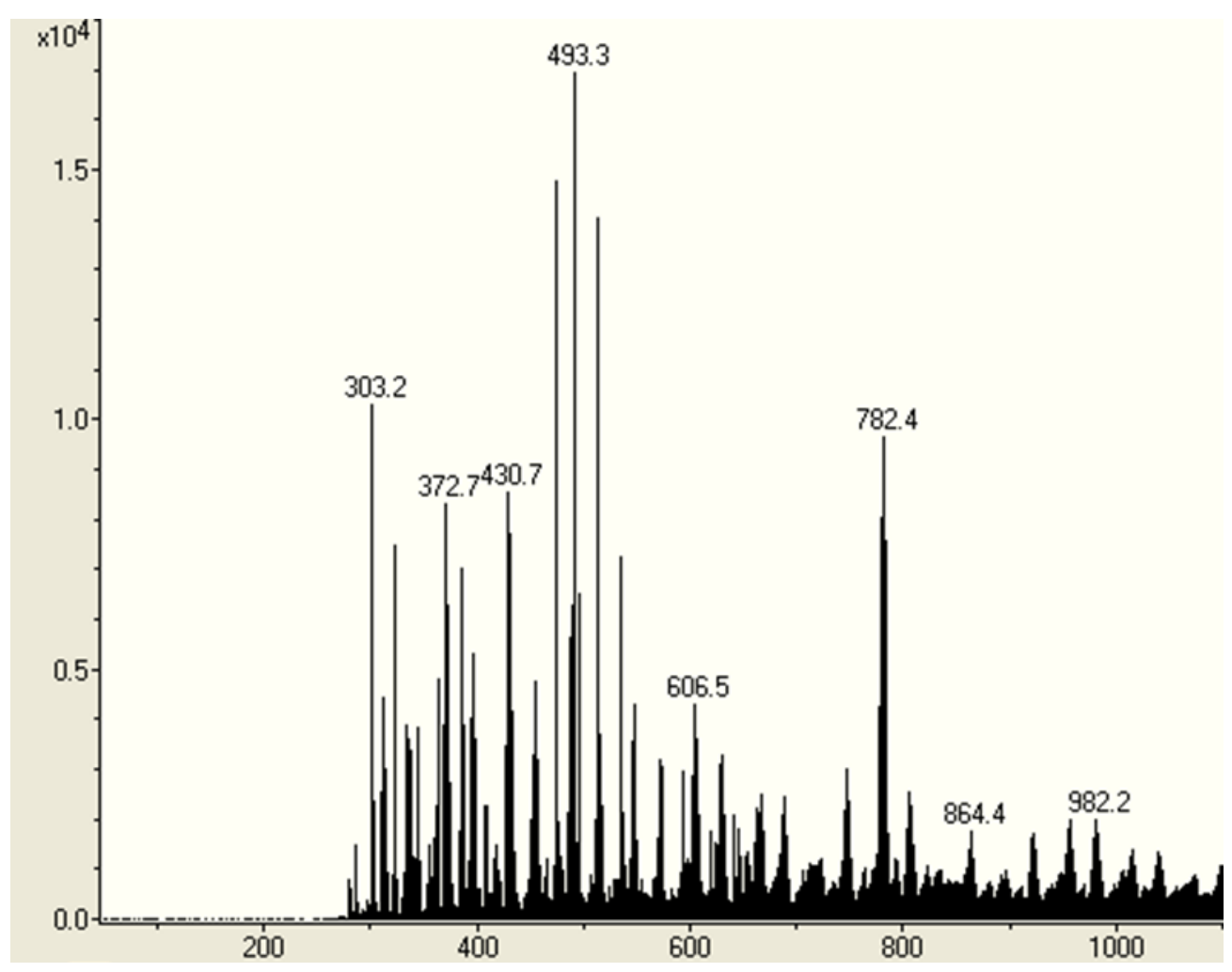

Spectrum 32: Mass spectrum of Au-CKAFKRKCoum:C(KAFKRK) $)_{3}$ (50:50) in PBS after enzymatic degradation. 


\section{SUPPLEMENTARY MATERIAL}

A CD accompanying this dissertation contains the following:

Text of Chapters 1-6 and Appendix 1. 